\title{
Identifying stage-specific markers of Alzheimer's disease using quantitative proteomics
}

\author{
Dissertation \\ for the award of the degree \\ "Doctor rerum naturalium" \\ of the Georg-August-Universität Göttingen
}

within the doctoral program Molecular Biology

of the Georg-August University School of Sciences (GAUSS)

submitted by

Oleksandr Yagensky

from

Lutsk, Ukraine

Göttingen, 2018 


\section{Thesis advisory committee:}

Supervisor, reviewer:

\section{Dr. John Jia En Chua}

Research Group Protein Trafficking in Synaptic Development and Function

Max Planck Institute for Biophysical Chemistry, Göttingen, Germany

Interactomics and Intracellular Trafficking Laboratory, Department of Physiology, Yong Loo Lin School of Medicine; Neurobiology/Ageing Programme National University of Singapore, Singapore, Singapore

TAC member, reviewer:

\section{Dr. Dieter Klopfenstein}

III Physical Institute,

Georg-August University of Göttingen, Göttingen, Germany

\section{TAC member:}

\section{Prof. Dr. Dirk Görlich}

Department of Cellular Logistics

Max Planck Institute for Biophysical Chemistry, Göttingen, Germany

\section{Extended evaluation committee:}

\section{Prof. Dr. Ahmed Mansouri}

Research Group Molecular Cell Differentiation

Max Planck Institute for Biophysical Chemistry, Göttingen, Germany

\section{Prof. Dr. Tiago Fleming Outeiro}

Department of Experimental Neurodegeneration

University Medical Center Göttingen, Göttingen, Germany

\section{Dr. Halyna Shcherbata}

Research Group Gene Expression and Signaling

Max Planck Institute for Biophysical Chemistry, Göttingen, Germany

Date of oral examination: 26.06.2018 
I hereby declare that I prepared this PhD thesis, entitled "Identifying stage-specific markers of Alzheimer's disease using quantitative proteomics", on my own and with no other sources and aids than those cited.

Oleksandr Yagensky

April 2018, Göttingen 
Dedicated to Daughter of the Moon 


\section{Table of Contents}

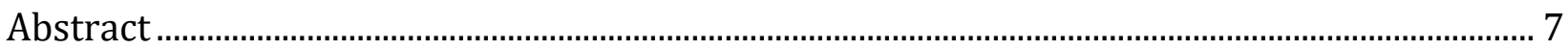

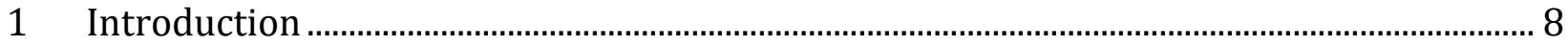

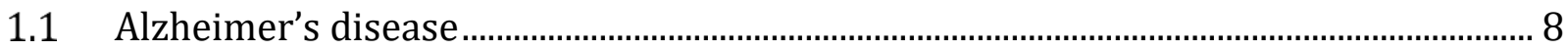

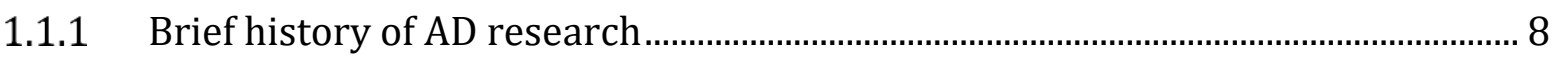

1.1.2 Neuropathological hallmarks of AD ........................................................................ 8

1.1.2.1 Amyloid beta plaques........................................................................................ 9

1.1.2.2 Neurofibrillary tangles ...................................................................................11

1.1.3 Progression and early diagnosis. ...........................................................................12

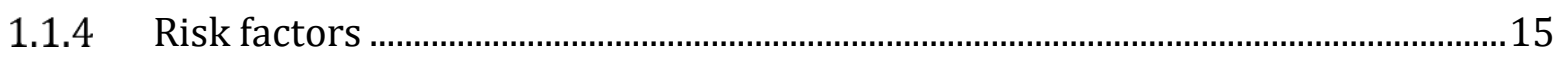

1.1.4.1 Genetic risk factors .......................................................................................16

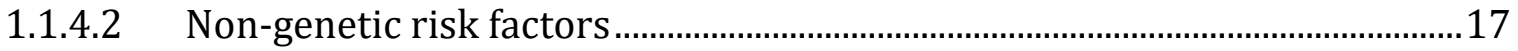

1.1.5 Biological processes related to AD progression....................................................18

1.1.5.1 Mitochondria dysfunction and oxidative stress .............................................18

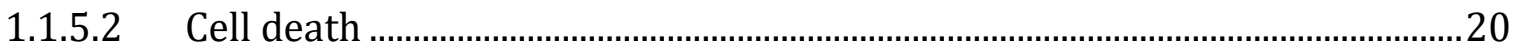

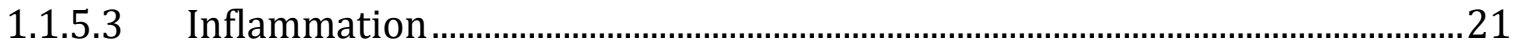

1.1.5.4 Cerebral amyloid angiopathy.......................................................................22

1.1.5.5 Metal ions and heme metabolism.....................................................................23

1.1.5.6 Other processes................................................................................................23

1.1.6 Therapeutic strategies.........................................................................................24

1.1.6.1 Acetylcholine esterase inhibitors and NMDA antagonists ..............................24

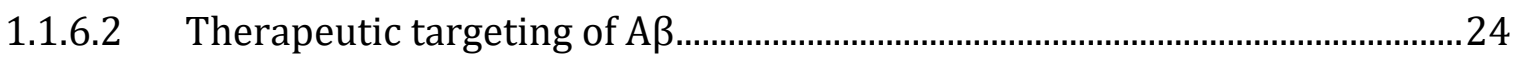

1.1.6.3 Anti-tau therapeutics ...............................................................................25

1.1.6.4 Other therapeutic strategies and perspectives ...............................................26

1.2 Animal models of Alzheimer's disease ………………………………………………....26

1.2.1 Spontaneous and interventional models ...............................................................27

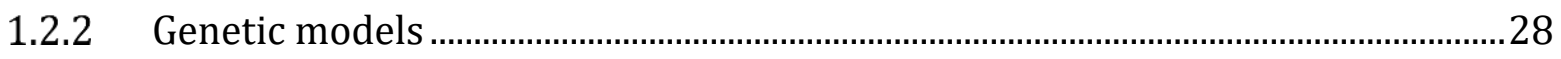

1.2.2.1 General considerations...............................................................................28

1.2.2.2 APP overexpression models .............................................................................29

1.2.2.3 APP/PSEN double transgenic models …………………………………….....30

1.2.2.4 Mouse models utilizing non-FAD transgenes .....................................................31 


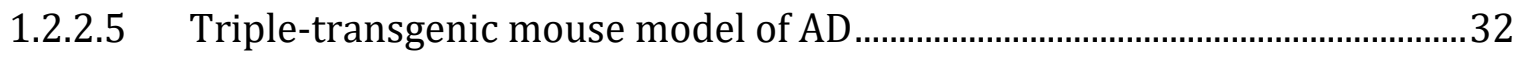

1.3 High-throughput profiling of gene and protein expression in AD ...............................33

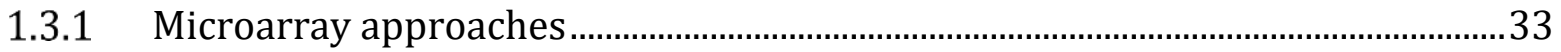

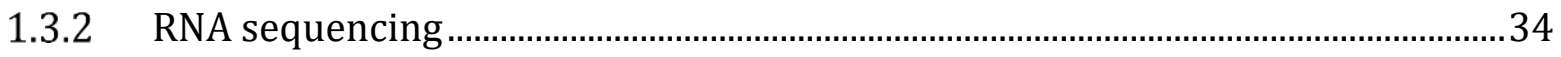

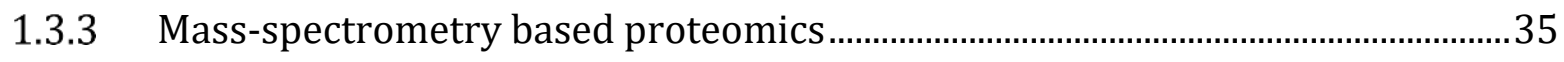

1.3.4 Assessment of posttranslational modifications ….............................................................38

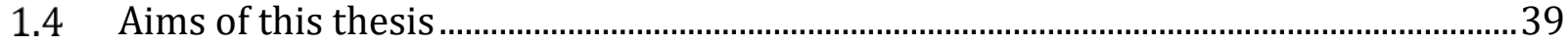

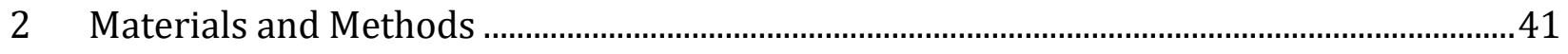

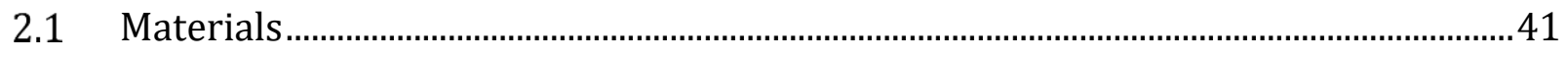

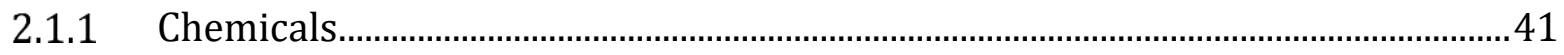

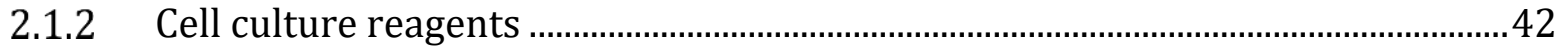

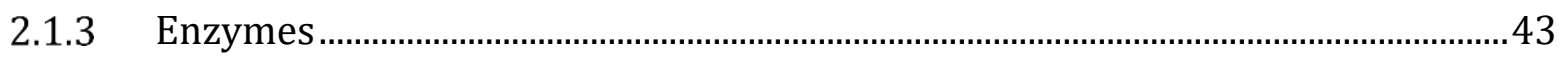

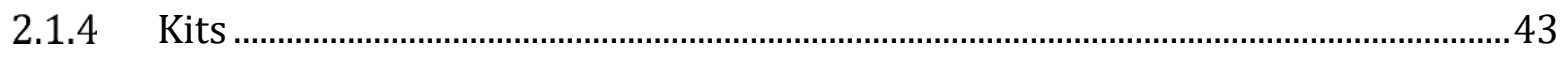

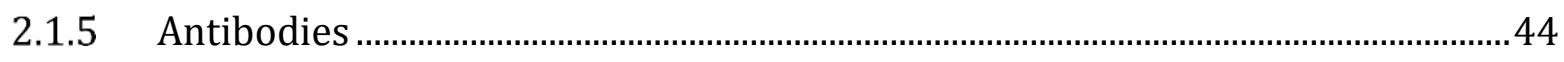

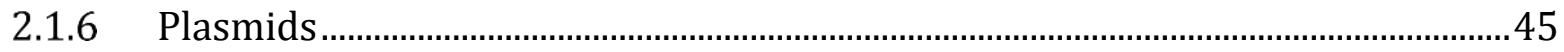

2.1.7 sgRNAs used for CRISPR/Cas9 mediated gene knockout.......................................46

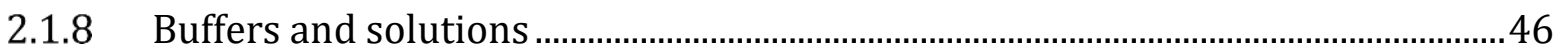

2.1.9 Cell culture media and solutions …........................................................................ 47

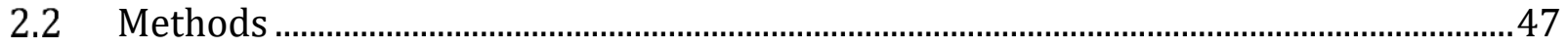

2.2.1 Mice

2.2.2 Preparation of soluble brain protein fraction ............................................................48

2.2.3 Measurement of protein concentration...................................................................... 49

2.2.4 Preparation of samples for mass spectrometry ..........................................................49

2.2.5 Measurements of mass spectrometry samples .......................................................... 49

2.2.6 Data processing and bioinformatics analysis ............................................................. 50

2.2.7 Analysis of human mRNA expression datasets........................................................52

2.2.8 Postmortem human brain samples .............................................................................. 52

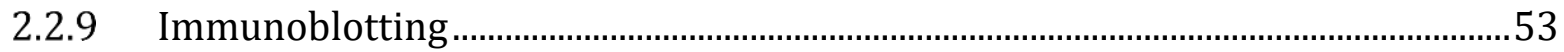

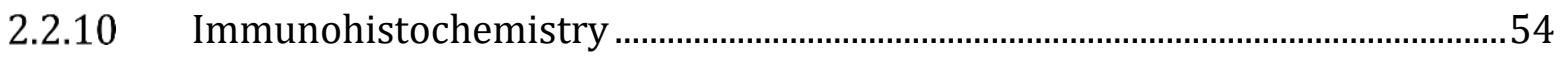

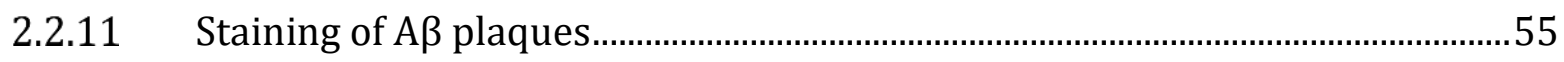

2.2.12 Primary neurons and cell culture ........................................................................5

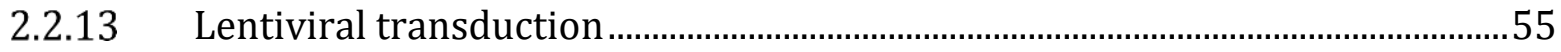




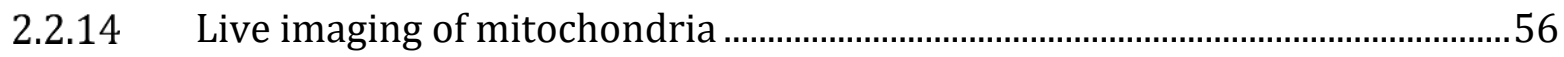

2.2.15 Identification of Hebp1 binding partners.............................................................56

2.2.16 Generation of Hebp1 knockout HeLa lines by CRISPR/Cas9 system.................57

2.2.17 In vitro cell-based assays ....................................................................................5

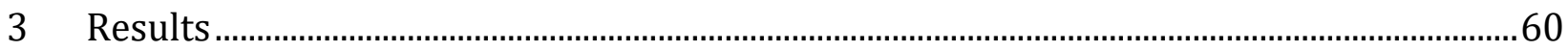

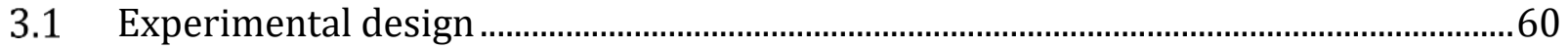

3.2 Assessment of brain proteome in 3×Tg-AD mice along AD progression .....................62

3.2.1 Principal component analysis clusters proteins by age and disease status.......64

3.2.2 Relative quantification of protein expression ............................................................64

3.2.3 Relating proteome changes to biological function ..................................................67

3.3 Alterations of brain phosphoproteome in AD progression ...........................................70

3.4 Putative presymptomatic markers of Alzheimer's disease .............................................75

3.4.1 Identification of proteins involved in early stage of AD ...........................................75

3.4.2 Expression of identified presymptomatic markers in postmortem brain samples from AD patients.......................................................................................................77

3.5 Investigation of heme-binding protein 1 and its potential functions in $\mathrm{AD}$ pathogenesis ............................................................................................................................79

3.5.1 Hebp1 is a neuronal protein upregulated in the brain of $3 \times \mathrm{Tg}$-AD mouse ........80

3.5.2 Hebp1 interacts with the mitochondrial contact site complex ……………….......81

3.5.3 Hebp1 facilitates heme-mediated cytotoxicity .......................................................... 84

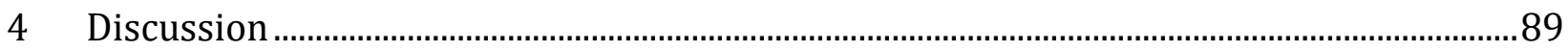

4.1 Analysis of biological processes affected in the brain of 3×Tg-AD mice .....................89

4.1.1 Regulation of microtubule stability .........................................................................

4.1.2 mRNA processing ................................................................................................92

4.1.3 Inhibition of serine proteases .............................................................................

4.1.4 Cell death ..............................................................................................................94

4.2 Heme-binding protein 1 as a novel candidate involved in early stages of AD

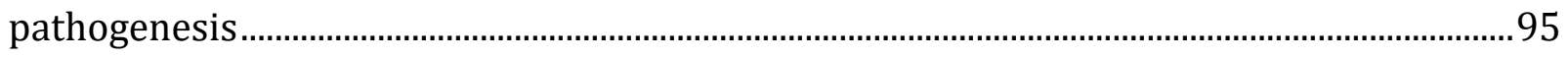

4.2.1 Association of Hebp1 with MICOS complex ...........................................................95

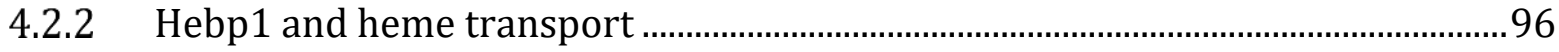

4.2.3 Hebp1 and cell death ............................................................................................97

4.2.4 Proteolytic cleavage of Hebp1 in immune response ……………...........................99

4.3 Advantages and caveats of applied experimental procedures ......................................99 


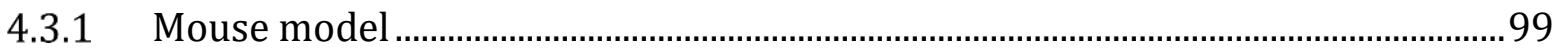

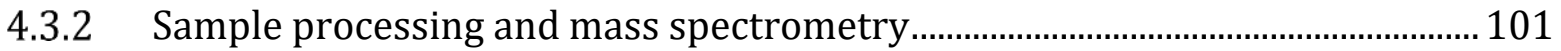

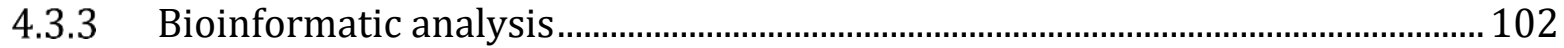

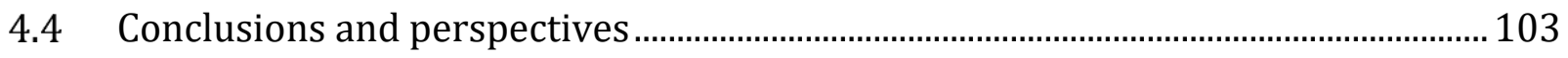

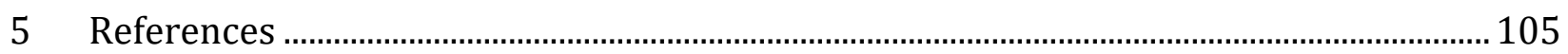

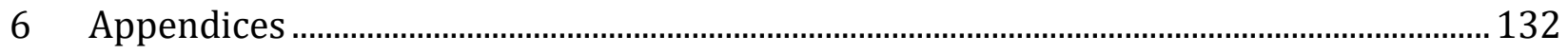

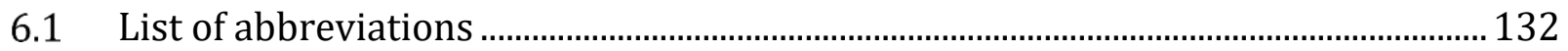

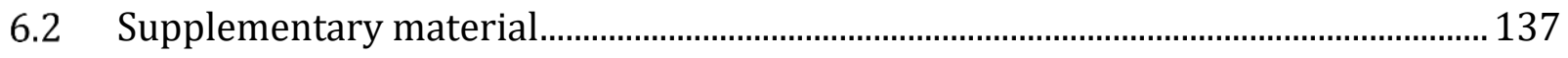

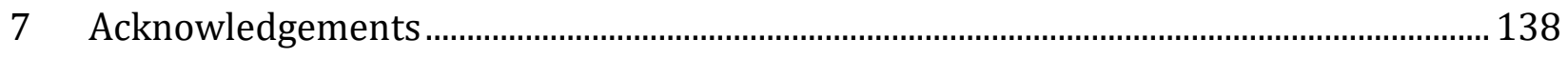

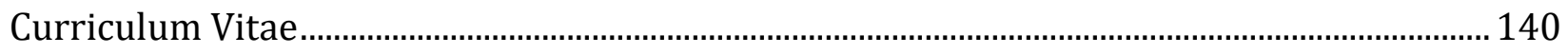




\section{List of Figures}

Figure 1-1. Neuropathological features of AD ............................................................................. 9

Figure 1-2. Proteolytic processing of amyloid precursor protein. …………………………….....10

Figure 1-3. Structure and phosphorylation of tau protein............................................................12

Figure 1-4. Progression of AD and available biomarkers. ............................................................14

Figure 1-5. The sequence of major pathogenic events leading to AD proposed by the

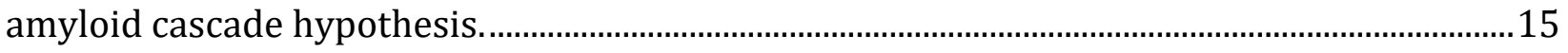

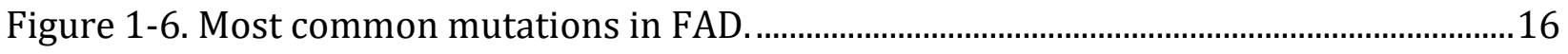

Figure 1-7. Neuropathology in 3×Tg-AD mouse model................................................................32

Figure 2-1. Preparation of soluble protein fraction from mouse brain.........................................48

Figure 3-1. Assessment of pathology in 3×Tg-AD mice used in this study...............................61

Figure 3-2. Experimental design. ................................................................................................62

Figure 3-3. Number of identified proteins and technical reproducibility of proteomic data.

Figure 3-4. Principal component analysis reveals disease- and age-dependent alterations of $3 \times$ Tg-AD brain proteome.

Figure 3-5. Comparative proteome analysis of $3 \times \mathrm{Tg}-\mathrm{AD}$ and control samples at different stages of AD.

Figure 3-6. Inference of activated biological processes in the progression of $\mathrm{AD}$ in $3 \times \mathrm{Tg}-\mathrm{AD}$

mice. .68

Figure 3-7. Identification of top upstream regulators in the proteomic dataset.

Figure 3-8. Analysis of brain phosphoproteome at presymptomatic and late stages of AD in $3 \times \mathrm{Tg}-\mathrm{AD}$ mice.

Figure 3-9. SRRM1 and 2 are phosphorylated at numerous phosphosites in the late stage of $\mathrm{AD}$ in $3 \times \mathrm{Tg}-\mathrm{AD}$ mice.

Figure 3-10. Canonical signaling pathways and kinase families affected in $3 \times \mathrm{Tg}-\mathrm{AD}$ at the presymptomatic and late stage of the disorder. .73

Figure 3-11. Putative early markers of Alzheimer's disease.

Figure 3-12. Hebp1 and Glo1, identified presymptomatic markers in 3×Tg-AD model, exhibit increased expression in brains of patients with rapidly-progressing forms of AD....78 Figure 3-13. Hebp1 expression in publicly available transcriptome databases of Alzheimer's disease. 
Figure 3-14. Analysis of Hebp1 expression in the brain of 3×Tg-AD mice. .80

Figure 3-15. Subcellular localization of Hebp1.

Figure 3-16. Hebp1 demonstrates perimitochondrial localization in cultured hippocampal neurons

Figure 3-17. Hebp1 interactome reveals its association with mitochondrial contact site and cristae organizing system (MICOS) complex...... .84

Figure 3-18. Validation of Hebp1 knockout in CRISPR/Cas9-edited clonal HeLa cell lines...85

Figure 3-19. Hebp1 is required for apoptotic response upon heme overload. .86

Figure 3-20. Measurement of oxidative stress and mitochondrial membrane potential in hemin-treated wild type and Hebp1-KO HeLa cells. .87

Figure 4-1. Hypothetical role of Hebp1 in heme metabolism. .97

\section{List of Tables}

Table 1-1. The most common promoters in mouse models of AD. 29

Table 2-1. List of chemicals used in this study.

Table 2-2. List of cell culture reagents used in this study. 42

Table 2-3: List of enzymes used in this study. 43

Table 2-4: List of used kits. 43

Table 2-5: List of used antibodies. 44

Table 2-6: List of plasmids used in this study. 45

Table 2-7: List of sgRNAs for CRISPR/Cas9 experiments. 46

Table 2-8: List of buffers and solutions with their composition used in this study. 46

Table 2-9: List of cell culture media and solutions with their composition used in this study.

Table 2-10. Patient details .53

Table 3-1. Putative presymptomatic brain markers of AD in literature. 76

Table 4-1. Phosphorylation profile of tau at time point 18 months. 91 


\section{Abstract}

Alzheimer's disease (AD) is a devastating neurodegenerative disorder that leads to progressive memory loss and impairment of other cognitive functions. It is the most prevalent form of dementia in the elderly and is estimated to affect 20 to 45 million people worldwide. The incidences are expected to rise sharply over the coming decades with no effective therapeutics available to combat the disease. Recent advances in AD research uncovered many important aspects of the disorder. Nevertheless, the AD progression at the molecular level, particularly at its early stage, remains elusive.

In this thesis I investigated the changes in the brain proteome and phosphoproteome over the course of neurodegeneration in a triple transgenic mouse model of $A D(3 \times \mathrm{Tg}-\mathrm{AD})$. Bioinformatic analysis of stage-specific alterations in protein expression and phosphorylation allowed to determine the affected biological functions along the progression of the disorder. Notably, proteins related to apoptotic response, mitochondria function and synaptic transmission were among the most affected groups in the early stages of AD.

Several proteins in the dataset exhibited strong expression change before the $\mathrm{AD}$ onset in $3 \times \mathrm{Tg}$-AD mice. These proteins can be considered as putative presymptomatic brain markers of $\mathrm{AD}$ and pose a special interest for their potential in early diagnosis and treatment of $\mathrm{AD}$. Closer investigation of one such marker, heme-binding protein 1 (Hebp1), revealed its increased expression in the brains of patients affected by rapidly-progressing forms of AD. Furthermore, Hebp1 is found to be expressed predominantly in neurons where it exhibits a perimitochondrial localization and interacts with the mitochondrial contact site and cristae organizing system (MICOS) complex. Remarkably, genetic depletion of Hebp1 reduces apoptosis induced by excessive levels of heme. Importantly, abnormalities in heme metabolism and disturbance of brain vasculature were previously reported in AD. Collectively, my findings suggest that the increase in Hebp1 expression early in AD progression can be linked to impaired heme metabolism and neuronal loss. 


\section{Introduction}

\subsection{Alzheimer's disease}

Alzheimer's disease (AD) is a progressive neurodegenerative disorder that gradually impairs memory, cognition and ability to perform simple daily tasks. AD is the most prevalent form of dementia in elderly, with its incidence increasing exponentially with age ${ }^{1}$. Estimates have indicated that 20 to 45 million people are currently affected by AD worldwide ${ }^{2,3}$. These numbers are expected to triple by $2050^{3}$. None of the available therapeutics have been able to stop and reverse AD. Aside from detrimental impact on the health of afflicted individuals, $\mathrm{AD}$ also poses a heavy economic burden. The aggregate cost of AD-related health care in 2017 in the USA alone has reached $\$ 259$ billion and is projected to grow to one trillion dollars per annum over the next three decades. Collectively, these statistics portray AD as one of the biggest socioeconomic challenges of the $21^{\text {st }}$ century. Deeper understanding of the mechanisms contributing to the disease and its progression is therefore vital for developing successful therapeutics that will contribute to a solution to this problem.

\subsubsection{Brief history of $A D$ research}

Alzheimer's disease was first described in 1901 by the German physician Alois Alzheimer, who followed the case of a fifty-year-old female patient affected by rapidly progressive cognitive deficiency 4 . For many decades afterwards, AD was regarded only as a presenile form of dementia occurring exclusively in middle-aged population (45 - 65 years), while cases of dementia in elderly ( $>65$ years) were commonly attributed to physiological aging. It was not until the late 1970s that pathological findings in senile dementias were recognized to closely resemble the original findings of Alois Alzheimer 5,6 . The last three decades have seen an immense progress in fundamental and clinical research related to AD. These efforts have unraveled many important aspects of $\mathrm{AD}$ pathogenesis and helped to establish better methods for its diagnosis. Despite all these efforts, the origin and precise mechanism of the disease remain elusive.

\subsubsection{Neuropathological hallmarks of $A D$}

Original study of Alois Alzheimer revealed the presence of unusual fibrillary tangled structures in the postmortem brain of the first AD patient ${ }^{4}$. These structures are now recognized as hallmarks of $\mathrm{AD}$ pathology. We now know that these are two types of protein 

1).
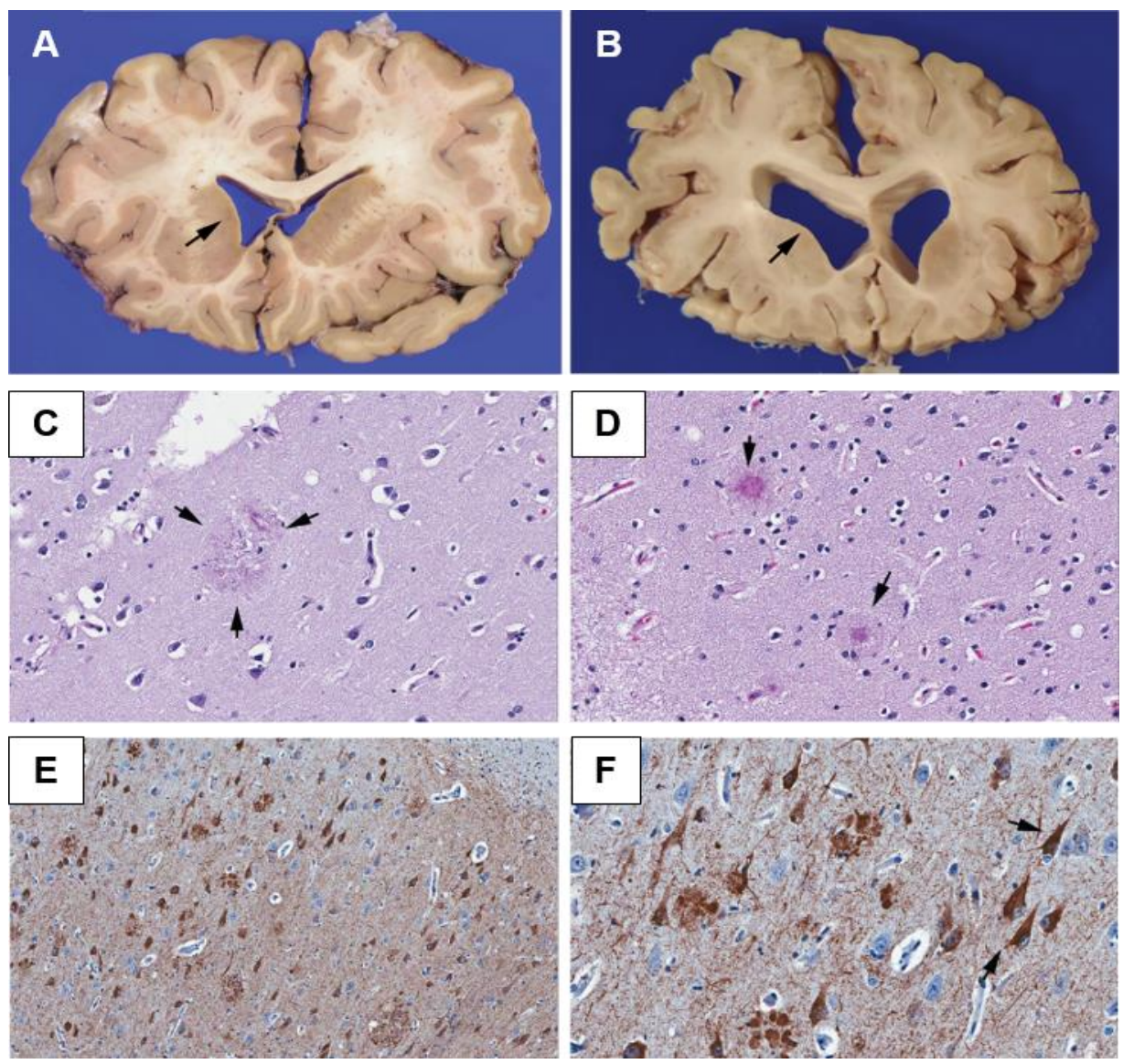

Figure 1-1. Neuropathological features of AD.

Coronal slices of fixed brain from two different patients, one without dementia (A) and one with (B); slices are at comparable coronal levels (near the head of the caudate nucleus). Arrows indicate a relatively normal lateral ventricle in the control case (A) versus a markedly enlarged lateral ventricle in the Alzheimer's disease subject (B). Cortical thinning is less prominent.

(C, D) Senile plaques. Hematoxylin and eosin-stained sections show a neuritic plaque without a prominent amyloid core (C, arrows) and two plaques with prominent amyloid cores (A $\beta$ plaques) (D, arrows). (E, F) Neurofibrillary tangles. Sections were immunostained with primary antibody against phospho-tau and visualized at low (E) and high (F) magnification. Arrows in panel F highlight immunoreactive neurons with classic flame-shaped NFT morphology.

Adapted from Vinters, 20157.

\subsubsection{Amyloid beta plaques}

$A \beta$ plaques are composed of aggregated $A \beta$ peptides (36-43 amino acids) that are produced by proteolytic processing of amyloid precursor protein (APP) ${ }^{8}$. APP is a transmembrane protein, which in neurons is presumably responsible for cell growth and synaptogenesis 9,10 . Nevertheless, the exact role of APP in brain development and function is not fully understood. 
APP is produced in neurons in large quantities and undergoes rapid turnover ${ }^{11}$. The proteolytic degradation of APP can occur through amyloidogenic or nonamyloidogenic pathway ${ }^{12}$. During nonamyloidogenic processing, APP is sequentially cleaved by the plasma membrane proteases $\alpha$ - and $\gamma$-secretase. These cleavages result in generation of a long soluble N-terminal fragment (APPs $\alpha$ ), a cytosolic C-terminal AICD fragment and a short soluble P3 peptide (Figure 1-2). In amyloidogenic pathway, APP is processed first by $\beta$ secretase in the endosomal compartment followed by $\gamma$-secretase cleavage typically at the plasma membrane. As a result, short $A \beta$ peptides are produced and released into the extracellular space (Figure 1-2). An alternative route for $A \beta$ trafficking to trans-Golgi network and subsequent intracellular accumulation of the peptide has been proposed as well 13,14 .

Strong hydrophobicity of $A \beta$ peptide makes it highly prone to aggregation ${ }^{15}$. With aging, $A \beta$ accumulates in brain, both intracellularly and extracellularly ${ }^{16}$. Extracellular accumulation of $A \beta$ deposits results in formation of characteristic plaques that have been long believed to be the main source of neurotoxicity in $\mathrm{AD}^{17}$. However, recent evidence suggest that oligomeric forms of $A \beta$ can be even more toxic than plaques ${ }^{18}$.
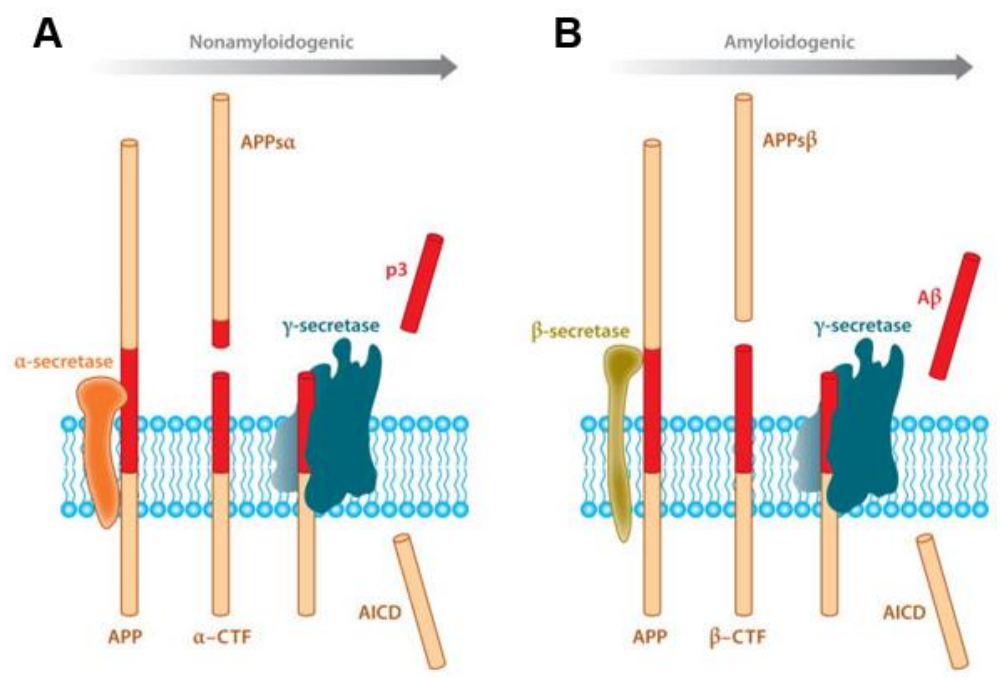

Figure 1-2. Proteolytic processing of amyloid precursor protein.

Sequential cleavage of the amyloid precursor protein (APP) occurs by two pathways. (A) Nonamyloidogenic processing of APP involving $\alpha$-secretase followed by $\gamma$-secretase is shown. (B) Amyloidogenic processing of APP involving BACE1 followed by $\gamma$-secretase is shown. Both processes generate soluble ectodomains (sAPPs $\alpha$ and sAPPs $\beta$ ) and identical intracellular C-terminal fragments (AICD).

Reproduced from $O^{\prime}$ Brien and Wong, 201412. 


\subsubsection{Neurofibrillary tangles}

The second distinctive neuropathological feature of AD are neurofibrillary tangles. NFTs consist primarily of hyperphosphorylated tau protein that under physiological conditions binds axonal microtubules and regulates their stability ${ }^{19}$. The density of tau tangles in the brain correlates strongly with the extent of dementia in individuals afflicted by AD $^{20}$. Six isoforms of tau protein are expressed in human brain ${ }^{21}$ (Figure 1-3). Each tau isoform consists of an acidic N-terminal region followed by a proline-rich motif (this forms the projection domain) and three or four tubulin-binding domains at the C-terminus ${ }^{22}$. Projection domain contains an internally disorganized structure that can interact with various cytosolic and membrane proteins ${ }^{23}$. This domain also determines distances between tightly packed axonal microtubules and therefore impacts axon diameter ${ }^{24}$. Tau binds microtubules via repeated domains (R1-R4) that consist of eighteen highly conserved amino acids. Binding of tau to microtubules increases the rate of microtubule polymerization and stabilizes their structure 25,26 .

The proper organization of microtubular network in axon is critical for intracellular protein trafficking and maintenance of synaptic activity ${ }^{27}$. These processes get strongly affected in AD largely due to conformational modifications of tau and hence inefficient maintenance of microtubule cytoskeleton ${ }^{28}$. The conformational changes of tau are caused predominantly by altered profile of post-translational modifications, mainly phosphorylation. The longest tau isoform (441 aa) contains almost 80 potential serine and threonine phosphosites. Most of them are located in proline-rich region and C-terminus of the protein. Few phosphosites also localize to microtubule binding repeats where they are a part of KXGS motif ${ }^{22}$. Under physiological conditions, a moderate level of tau phosphorylation is required for its efficient interaction with microtubules ${ }^{29}$. However, in AD tau gets heavily hyperphosphorylated ${ }^{30}$. Abnormal activity of protein kinases and phosphatases is a key contributor to the altered phosphorylation profile of tau in AD. Glycogen synthase kinase 3 beta (GSK-3 3 ), cAMPdependent protein kinase (PKA), cyclin-dependent kinase 5 (Cdk5) and other kinases have been demonstrated to be involved in hyperphosphorylation of tau ${ }^{31,32}$. Hyperphosphorylated tau loses its affinity to tubulin, leading to breakdown of microtubule networks ${ }^{33}$. Moreover, it facilitates detachment properly phosphorylated tau molecules and other microtubule associated proteins (MAPs) from microtubules further exacerbating the damage ${ }^{34,35}$. 
Hyperphosphorylated tau is prone to aggregation and after initial accumulation forms neurofibrillary tangles in the neurons of $A D$ patients. As in case of $A \beta$ accumulation, it takes long time (presumably years or decades) for aggregated tau to provoke a cytotoxic response ${ }^{21}$.

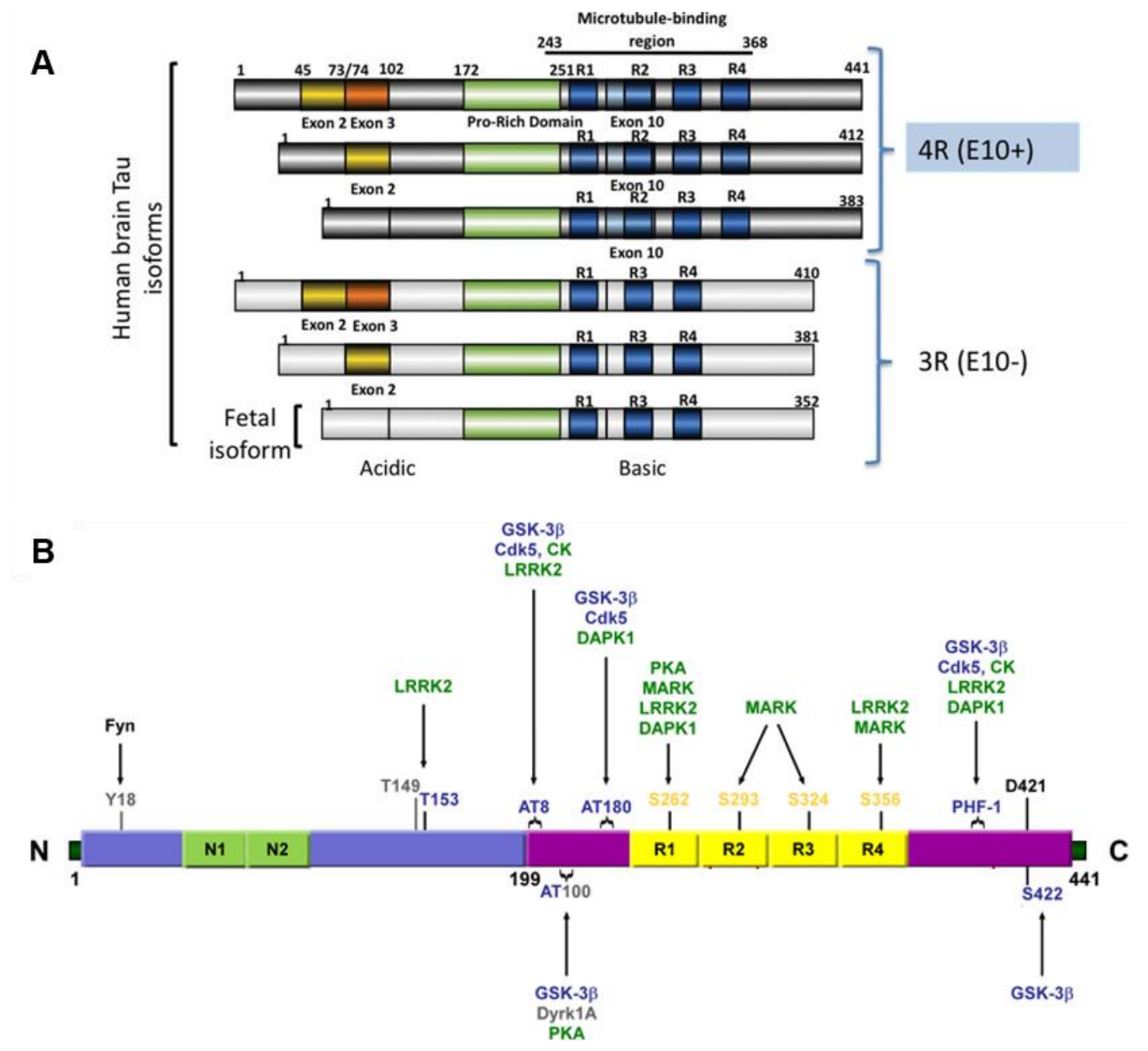

Figure 1-3. Structure and phosphorylation of tau protein.

(A) Schematic representation of the six tau isoforms in human brain.

(B) Schematic representation of phosphorylation sites on the longest tau isoform. SP/TP motifs (represented in blue), KXGS motifs (represented in yellow), and other sites (represented in gray) can be phosphorylated by proline-directed kinases (represented in blue) and non-proline directed Ser/Thr kinases (represented in green). AT8, AT100, AT180 and PHF-1 are classical antibody epitopes used for detection of hyperphosphorylated tau.

Compiled and adapted from Buée et al., $2010^{21}$ and Tenreiro et al., $2014^{32}$.

\subsubsection{Progression and early diagnosis.}

According to the latest recommendations of Alzheimer's society, the progression of AD in humans can be subdivided into three stages: preclinical AD, mild cognitive impairment (MCI) due to $\mathrm{AD}$ and dementia due to $\mathrm{AD}^{36}$. In $\mathrm{AD}$, pathophysiological transformations occur long 
before manifestation of clinical symptoms (Figure1-4). Many scientists argue that therapeutic intervention at presymptomatic stage can considerably slow down or stop the progression of AD. While MCI and dementia stages can be determined based on symptomatic development, diagnosis of $\mathrm{AD}$ at presymptomatic phase is not trivial and relies on brain imaging techniques and identification of biomarkers in cerebrospinal fluid (CSF) and blood.

Advancement in brain imaging techniques have allowed for comprehensive assessment of $A \beta$ aggregation in the brain of living individuals. Positron emission tomography (PET) scanning using (11)C-labelled Pittsburgh Compound-B ligand $\left(\left({ }^{11} \mathrm{C}\right) \mathrm{PIB}\right)$, a radiolabeled chemical capable of binding to $A \beta$ fibrils, could successfully detect accumulation of $A \beta$ aggregates in the cortex of $\mathrm{AD}$ patients but not age-matched controls or healthy young individuals ${ }^{37}$. Further application of PET scanning revealed that $A \beta$ deposits can already be detected even before the onset of clinical symptoms, prior to the emergence of $\mathrm{MCI}^{38,39}$. Brain imaging studies and autopsy experiments demonstrate that development of plaque pathology roughly precedes AD diagnosis by about 10 years ${ }^{40-42}$. However, it remains to be determined whether $\mathrm{A} \beta$ aggregation in cognitively healthy individuals is predictive of developing AD later in life.

Reduction of glucose metabolism is also related to $\mathrm{AD}^{43}$. Uptake of radio-labeled glucose (fluorodeoxyglucose 18F) can be assessed by PET scanning and serve as an early indication of the disease ${ }^{44}$. Another brain imaging technique that is implemented for early identification of $\mathrm{AD}$ is volumetric MRI scanning ${ }^{45}$. This method is based on the detection of abnormalities in brain structure and can identify early signs of neurodegeneration.

The analysis of molecular biomarkers from CSF or blood can also be a useful tool in AD diagnosis. A recent study of plasma biomarkers revealed a strong correlation between $A \beta$ levels in blood and CSF and amyloid burden of brain determined by PET ${ }^{46}$. Surprisingly, lower levels of $A \beta_{1-42}$ in CSF are associated with $\mathrm{AD}^{47}$. In contrast, elevated total and phospho-tau can be detected in CSF of AD patients ${ }^{47}$. Interestingly, increase in CSF phospho-tau coincides with the symptomatic onset of $\mathrm{AD}$ but altered level of $\mathrm{A} \beta 1-42$ precedes symptomatic development of the disease by few years ${ }^{48}$. This observation supports amyloid cascade hypothesis putting changes in $\mathrm{A} \beta$ production upstream of tau hyperphosphorylation in $\mathrm{AD}$ progression. 
Although statistically significant, the differences in CSF A $\beta$ and tau obtained in large cohort studies are rather small, limiting the usefulness of these biomarkers in routine diagnosis. There is thus a need to identify alternative biomarkers capable of detecting $\mathrm{AD}$ at the presymptomatic stage that are able to clearly discriminate between healthy and diseased individuals.
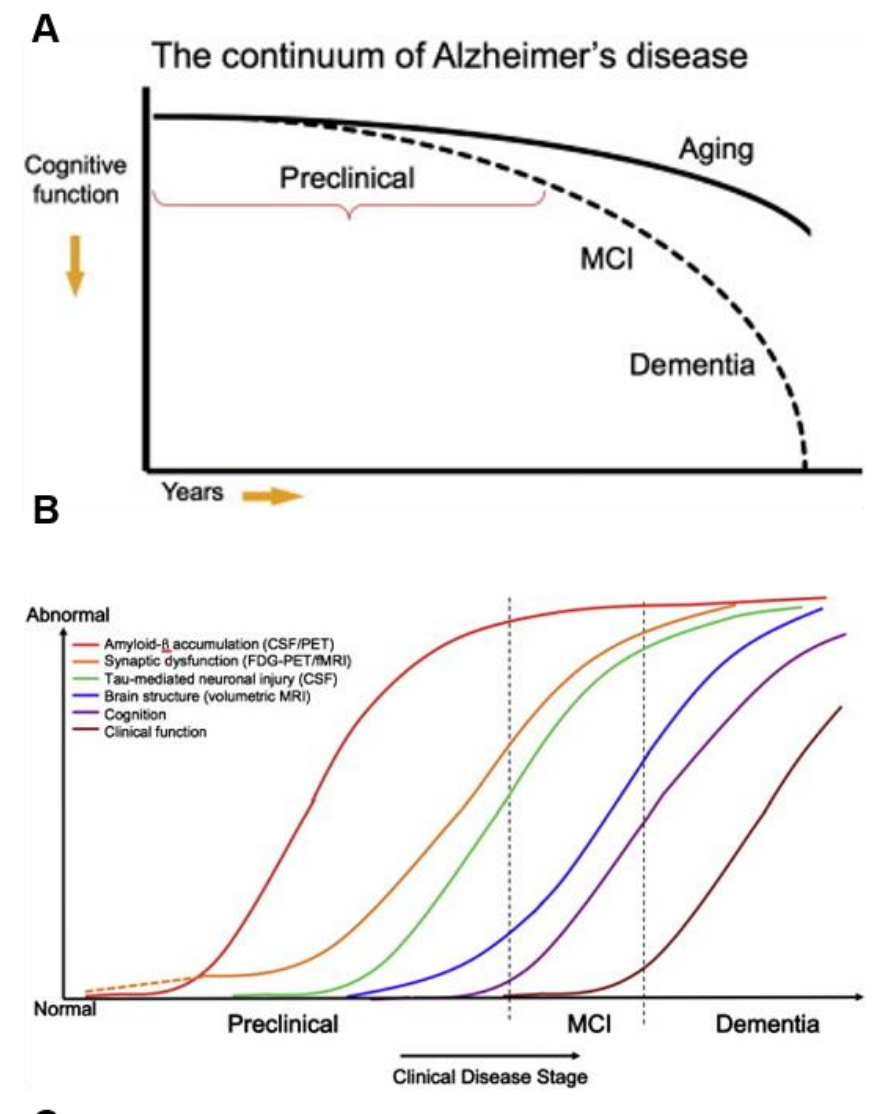

C

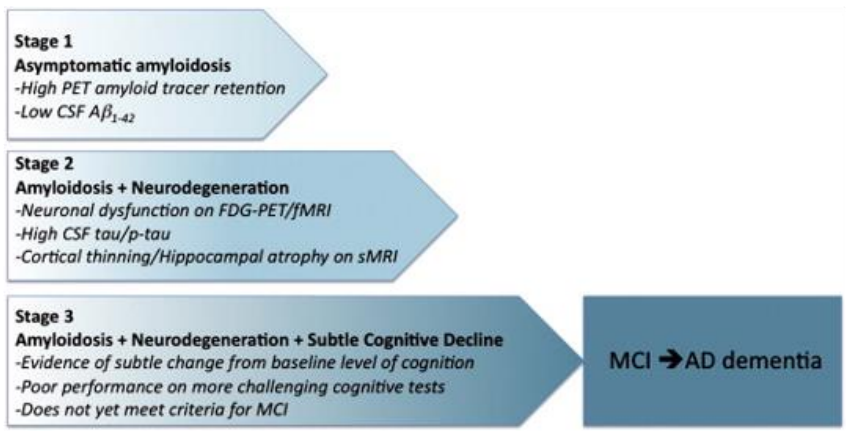

Figure 1-4. Progression of AD and available biomarkers.

(A) Model of the clinical trajectory of AD.

(B) Hypothetical model of dynamic biomarkers (description in the text).

(C) Graphic representation of the proposed staging framework for preclinical AD.

Taken and compiled from Sperling et al., 201136. 


\subsubsection{Risk factors}

Amyloid cascade hypothesis has been at the center of current understanding of $\mathrm{AD}^{49}$ (Figure1-5). According to this theory, increased generation of toxic $A \beta$ peptides in the brain is a primary cause of $\mathrm{AD}$ that leads to hyperphosphorylation and aggregation of tau, synaptic loss, neurodegeneration and finally cognitive decline.

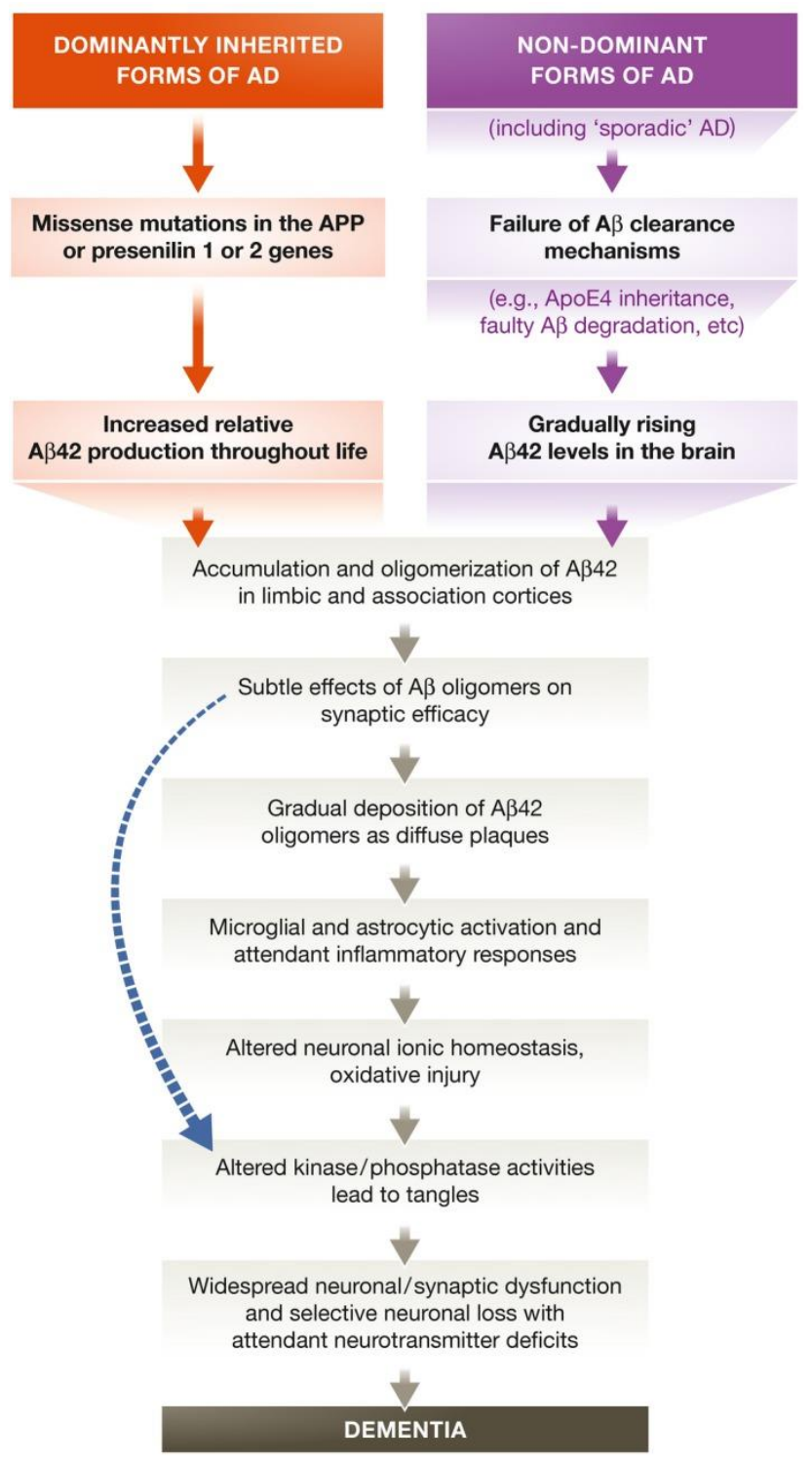

Figure 1-5. The sequence of major pathogenic events leading to AD proposed by the amyloid cascade hypothesis.

Taken from Selkoe and Hardy, $2016^{18}$.

Amyloid cascade hypothesis was proposed in 1992, and since then many evidence were published in its support ${ }^{18}$. Nevertheless, amyloid cascade hypothesis cannot fully explain 
several aspects of $\mathrm{AD}$ pathogenesis and has often been challenged in the scientific community ${ }^{50-52}$. A $\beta$ accumulation is necessary but not sufficient to produce the clinical symptoms of MCI and dementia ${ }^{53}$. That is why it is important to understand the factors (both related and unrelated to $A \beta$ ) that can bring about pathological changes in $A D$.

\subsubsection{Genetic risk factors}

The mutations in genes involved in APP processing result in inherited early-onset AD that typically affect individuals under the age of 65. These cases are known as familial Alzheimer's disease or FAD and make up around $1 \%$ of all $\mathrm{AD}$ cases ${ }^{54}$. Mutations in the following three genes have causative relationship with FAD: APP, PSEN1 and PSEN2. PSEN1 and PSEN2 encode two components of the $\gamma$-secretase complex, presenilin-1 and 2, respectively. Thirtytwo mutations for APP, 179 for PSEN1 and 14 for PSEN2 have been identified so far (Figure 1-6).
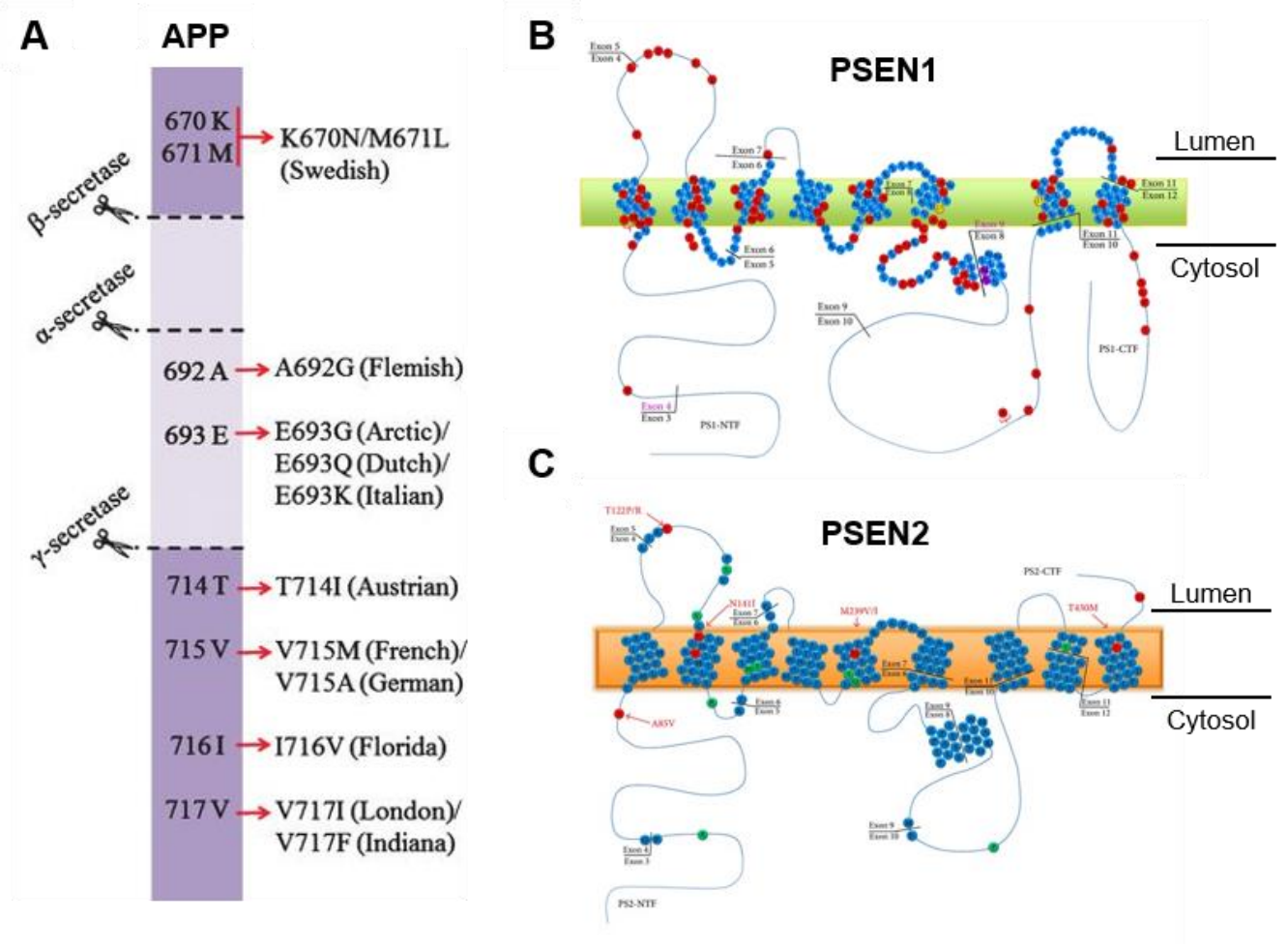

Figure 1-6. Most common mutations in FAD.

(A) Most common APP mutations and their locations. Numbering refers to the longest APP isoform (APP770). Schematic representation of PSEN1 (B) and PSEN2 (C) with the mutations occurring in FAD labeled in red. Compiled and adapted from Esquerda-Canals et al., 201755 and Meraz-Ríos et al., 201456. 
Mutations of $A P P$ were named after the geographical region where they were first discovered. The most famous Swedish mutation of APP occurs in proximity of $\beta$-secretase site, while other known mutations cluster around $\gamma$-secretase point of cleavage. Interestingly, individuals with trisomy of chromosome 21 (Down syndrome), possess additional copy of $A P P$ gene and are more susceptible to develop $\mathrm{AD}$ at a young age ${ }^{57}$. The $\mathrm{AD}$-related mutations in presenilins occur predominantly in their transmembrane domains. Typically, these mutations lead to increased activity of $\gamma$-secretase complex or shift of the cleavage site by a few amino acids that results in production of longer, more toxic forms of $A \beta^{56}$.

The vast majority of AD cases are sporadic (SAD) and according to the current knowledge are not predetermined by the presence of any fully penetrant variant of a single gene. Nevertheless, genetic risk factors of SAD do exist. Almost 700 different genes demonstrate some degree of association with $\mathrm{AD}^{54}$. $A P O E \varepsilon 4$ allele is the strongest genetic risk factor for SAD. In humans, APOE can be expressed from three different alleles $\varepsilon 2, \varepsilon 3$ and $\varepsilon 4$. The estimated prevalence of $\varepsilon 4$ allele in general population is about $13.7 \%$, while its frequency is almost tripled in AD patients $(36.7 \%)^{58}$. APOE is a primary cholesterol carrier in the brain that is involved in lipid metabolism and injury repair ${ }^{59}$. Expression of APOE from $\varepsilon 4$ allele has been linked to reduced clearance of $A \beta$ in the brain ${ }^{60,61}$. Remarkably, APOE $\varepsilon 4$ also affects AD pathogenesis through $\mathrm{A} \beta$-independent pathways that impact synaptic plasticity 62,63 , inflammatory response ${ }^{64}$ and glucose metabolism ${ }^{65}$. Interestingly, polymorphism in other genes involved in regulation of these processes is associated with AD. Few examples include: ACE (angiotensin 1) 66, CHRNB2 (cholinergic receptor nicotinic beta 2 subunit) ${ }^{67}$ and GAPDHS (Glyceraldehyde-3-Phosphate Dehydrogenase) ${ }^{68}$. The full list of genes and polymorphisms associated with $\mathrm{AD}$ can be found on publicly available Alzgene web resource (http://www.alzgene.org/).

\subsubsection{Non-genetic risk factors}

The strongest non-genetic risk factor for $\mathrm{AD}$ is age $\mathrm{e}^{3}$. Apart from aging, lifestyle also impacts predisposition to AD. Regular physical activity is associated with reduced risk of developing $\mathrm{AD}$ in old age ${ }^{69}$. Higher levels of cognitive and social activity during life also postpones the age of $\mathrm{AD}$ onset. For example, people with higher number of years spent on education are at lower risk for developing $\mathrm{AD}^{70}$. 
Several pathophysiological conditions have been linked to AD as well. For instance, a history of traumatic brain injury puts individuals under increased risk of acquiring $\mathrm{AD}^{71}$. Prevalence of $\mathrm{AD}$ in patients with type II diabetes is twice as high in comparison to individuals without the disease ${ }^{72}$. Many factors that worsen cardiovascular pathology such as smoking, obesity in midlife, high cholesterol and hypertension are also reported risk factors for $\mathrm{AD}^{73-76}$. These associations highlight that dysregulation of general metabolism creates a fertile environment for AD to blossom.

\subsubsection{Biological processes related to AD progression}

Over the last three decades, we have gotten a little closer towards understanding the AD at the molecular level. But how do the changes in individual genes and proteins contribute to the progression of the disease? To find the answer to this question, it is important to put the knowledge of molecular pathways in the context of brain physiology. In this section, major biological processes altered in $\mathrm{AD}$ will be reviewed, demonstrating that coordinated interplay between different molecular, cellular and systemic factors contributes to the development of the disease.

\subsubsection{Mitochondria dysfunction and oxidative stress}

Mitochondria are essential organelles that regulate neuronal metabolism, protein turnover, oxidative stress and cell death response. They are directly involved in progression of many neurodegenerative disorders ${ }^{77}$. Several aspects of mitochondria biology have been linked to AD.

One of them is mitochondria dynamics. Mitochondria occur not as stand-alone organelles but rather as a dynamically interchangeable moiety ${ }^{78}$. The dynamics of such mitochondrial network is regulated by the balance between mitochondrial fusion and fission ${ }^{79}$. This balance is disturbed in $\mathrm{AD}$ as indicated by altered expression of proteins regulating fusion/fission events $^{80}$. Stability of inner mitochondrial membrane is also important for mitochondria dynamics ${ }^{81}$. Proper positioning of mitochondria within the cells depends on the interaction of mitochondria with $\mathrm{ER}^{79}$. Communication between mitochondria and ER is particularly important for lipid metabolism, apoptosis and intracellular $\mathrm{Ca}^{2+}$-signaling. The ERmitochondria communication occurs through specialized membrane patches called mitochondria-associated ER membranes (MAM) ${ }^{82}$. Interestingly, components of $\gamma$-secretase 
(presenilins) are enriched on MAMs ${ }^{83}$. Moreover, the number of MAMs is upregulated in fibroblasts isolated from both FAD and SAD patients causing elevated $\mathrm{Ca}^{2+}$ concentrations in mitochondria, which may have a positive effect on the regulation of apoptotic response ${ }^{83,84}$. Mitochondrial proteostasis is another mitochondria-related process that is associated with neurodegenerative pathologies. Recently, decreased expression of proteins involved in mitochondrial unfolded protein response was reported in a large cohort of AD patients ${ }^{85}$. Remarkably, pharmacological enhancement of proteostasis led to significant amelioration of pathology in various animal models of $\mathrm{AD}^{85}$. This indicates that counterbalancing the degradation of mitochondrial proteins might be a viable therapeutic strategy to combat AD. On the other hand, inefficient removal of damaged mitochondria can exacerbate the neurodegenerative process. An important player in this process is PTEN-induced putative kinase (PINK1) which initiates degradation of mitochondria by induction of mitophagy ${ }^{86}$. A recent study demonstrates that overexpression of active PINK1 in mouse model of AD reduces $A \beta$ levels, oxidative stress and synaptic dysfunction ${ }^{87}$.

Remarkably, the relationship between mitochondria and $A \beta$ is directly related to mitochondria dynamics and protein degradation. As mentioned above, active $\gamma$-secretase can be found on MAMs suggesting potential cleavage of APP in proximity of the mitochondrial surface. Indeed, peri-mitochondrial $A \beta_{1-42}$ peptides were shown to inhibit import of mitochondrial proteins via co-aggregation ${ }^{88}$. $A \beta$ can also inhibit import and maturation of mitochondrial proteins by interference with mitochondrial peptidasome Cym1/PreP leading to perturbation of mitochondrial proteostasis ${ }^{89}$.

Mitochondria are the major intracellular source of reactive oxidative species (ROS). At the same time, antioxidative systems of mitochondria keep ROS levels in check and protect cells from oxidative stress ${ }^{90}$. Multiple studies have demonstrated that oxidative stress precedes $A \beta$ pathology in $A D$ patients and mouse models of the disease ${ }^{91,92}$. Dysregulation of glutathione (a key molecule involved in antioxidative response) metabolism was reported in $\mathrm{AD}$ and other neurodegenerative disorders ${ }^{93}$. Interestingly, reduced mitochondrial function and increased oxidative stress led to elevation of $A \beta$ and phospho-tau levels in $A D$ mice 94,95 . Combining these data with the knowledge of environmental risk factors, metabolic 
dysfunction can be seen as a potential trigger of $A D$ rather than just a consequence of $A \beta$ or tau accumulation.

Mitochondria are also essential for regulation of another biological process crucial in AD, namely apoptosis, which will be discussed in the next section.

\subsubsection{Cell death}

Cell death of neurons is a key event in AD. Early studies reported the loss of $10-30 \%$ of neurons in entorhinal cortex and hippocampus of AD patients already at the MCI stage ${ }^{96,97}$. A recent report has demonstrated that the rate of neuronal loss increases with the disease progression and correlates well with the Braak classification of AD staging ${ }^{98}$.

Cell death can happen by distinct mechanisms. The most studied pathways in neurodegeneration include apoptosis (programmed cell death), necrosis, excitotoxicity and autophagic cell death ${ }^{99}$. Examining mechanisms of cell death in brain in vivo has been challenging. Although the exact mechanism of neuronal cell death in AD is still debatable, most of the evidence indicate towards apoptosis being the prevailing pathway ${ }^{100}$.

Oxidative stress has a direct impact on apoptosis. The positive correlation was found between production of hydrogen peroxide and amyloid load of the brain ${ }^{101}$. This effect is caused by $A \beta$ itself as it is capable of reaching mitochondria where it can induce ROS production and cause cytotoxicity ${ }^{102,103}$. Localization of $A \beta$ to mitochondria induces cell death through apoptotic pathway as it increases expression of the pro-apoptotic protein Bax and reduces the levels of the anti-apoptotic Bcl-2104. $A \beta$ was also shown to interact with Cyclophilin-D, a component of mitochondria transition pore and a positive regulator of apoptosis ${ }^{105}$. Remarkably, depletion of Cyclophilin-D rescues impairment of mitochondria in $\mathrm{AD}$ mice and leads to improvement of learning memory. Increased expression of truncated scaffolding protein RanBP9 was reported in AD ${ }^{106}$. RanBP9 can activate cofilin (an activator of apoptotic response) through dephosphorylation suggesting a potential role of RanBP9 in initiation of the apoptotic response in $\mathrm{AD}^{106,107}$.

Neurons are not the only cells exhibiting higher rates of cell death in AD brain. Endothelial cells are particularly susceptible to apoptosis that results in disintegration of blood vessel, ischemia and inflammation ${ }^{108}$. Treatment of cultured endothelial cells with $A \beta_{1-42}$ peptides 
induced apoptosis via activation of death receptors DR4 and DR5109. This suggests that A $\beta$ mediated apoptosis follows different pathways in neurons and endothelial cells.

Inhibition of apoptotic cell death can be a viable path to reduce neurodegeneration. Overexpression of the anti-apoptotic protein Bcl-2 in triple transgenic mouse model reversed AD pathology, though it is still to be determined whether the observed effects were achieved exclusively due to interference with apoptosis ${ }^{110}$. Inhibition of oxidative stress and inflammation by pharmacological treatment can also reduce apoptosis and is considered as a potential therapeutic strategy to combat $\mathrm{AD}^{111,112}$.

\subsubsection{Inflammation}

Inflammation accompanies $\mathrm{AD}$ progression ${ }^{113}$. Innate immune response in the brain is mediated primarily by microglia, brain resident macrophages ${ }^{114}$. Microglia is recruited to $A \beta$ plaques in transgenic mice and has been shown to uptake and process $A \beta$ in lysosomes ${ }^{115}$. PET scanning of human brains revealed higher number of activated microglial cells in the brain of AD patients in comparison to age-matched controls ${ }^{116,117}$. Remarkably, the load of active microglia correlates positively with the cognitive decline but not amyloid burden of the brain in $\mathrm{AD}$ patients ${ }^{117}$. Role of other brain cell types in inflammation should not be overlooked. Astrocytes serve as a trophic support for neurons, maintain ion homeostasis and fine tune synaptic transmission ${ }^{118}$. Like microglia, astrocytes can be activated in response to changes in environment. Previous studies proposed that activation of astrocytes in response to inflammation leads to reduction of their neuro-supportive function and thus negatively impacts brain health in $\mathrm{AD}^{119}$. At the same time, reactive astrocytes can help clearing brain from $A \beta$ deposits and may be critical to reduce progression of neurodegeneration ${ }^{120}$. Although not studied extensively in this regard, oligodendrocytes and neurons may also contribute to neuroinflammation ${ }^{121,122 .}$

Experiments in vitro have demonstrated that $A \beta$ is capable of inducing the production of a wide range of proinflammatory cytokines through direct or indirect interactions with microglia ${ }^{123}$. For example, $A \beta$ is capable of binding Receptor for advanced glycation products (RAGE) on the surface of glia cells. This interaction exaggerates neuronal stress, accumulation of $A \beta$, memory impairments and inflammation ${ }^{124}$. Upregulation of proinflammatory cytokines such as TNF- $\alpha$, TGF- $\beta$ and IL-6 also strongly promotes gliosis and 
oxidative stress, but at the same time reduces amyloidosis in the mouse models of $A D^{125,126}$. The complement system is a centerpiece of innate immune response capable of recognizing molecular signatures of pathogens and injured tissues. Complement proteins are upregulated in the brains of $\mathrm{AD}$ patients where they can also be found in $\mathrm{A} \beta$ plaques ${ }^{127}$. Opposing roles of the complement system with regard to AD pathogenesis have been proposed. Activation of complement can ameliorate toxic $\mathrm{AD}$ environment, for example, by clearing up apoptotic cells in the brain ${ }^{128}$. On the other hand, complement system has been shown to mediate synapse elimination in central nervous system (CNS) ${ }^{129}$. Toll-like receptors (TLR) ${ }^{130}$, cyclooxygenase 2 (COX2) ${ }^{131}$ and nitric oxide synthase (NOS) ${ }^{132}$ are important mediators of immune response and have also been linked to AD. Recent studies indicate a strong association between risk for developing $\mathrm{AD}$ and a gene variant of TREM2, an immunoglobulin domain-containing transmembrane receptor that is expressed on the surface of microglia ${ }^{133}$.

Despite extensive studies, it is still not fully clear whether inflammation is a primary driver of $\mathrm{AD}$ or is just a secondary process activated in response to other factors. Both pro- and antipathogenic effects of inflammatory response were reported in AD. Based on the available data, it can be speculated that glia is activated in response to the pro-inflammatory stimulus to combat $\mathrm{AD}$, while neurons get caught up in collateral damage of inflammatory environment.

\subsubsection{Cerebral amyloid angiopathy}

Cerebral amyloid angiopathy (CAA) is a pathological process characterized by accumulation of amyloid fibrils around brain vasculature, typically within the outer basement membrane ${ }^{134}$. CAA accompanies AD progression with about $80-90 \%$ of AD patients affected by this disorder ${ }^{135}$. CAA leads to increased permeability of blood brain barrier (BBB) and local disruption of vasculature ${ }^{136}$. Microbleeds, which are a consequence of CAA, are more prevalent in $\mathrm{AD}$ than in the general population and are associated with increased mortality 137,138 . Leakage of vasculature was proposed as one of the major factors that links neurodegeneration to aging ${ }^{139}$. Leaky BBB allows systemic factors of aged individuals to penetrate the brain environment easier where they can induce inflammation and exacerbate AD-related damage. 


\subsubsection{Metal ions and heme metabolism}

Metal ions contribute strongly to protein misfolding and related neurodegenerative diseases ${ }^{140} . \mathrm{Cu}^{2+}$ and $\mathrm{Zn}^{2+}$ were seen to be concentrated in $\mathrm{A} \beta$ plaques of $\mathrm{AD}$ patients ${ }^{141}$. Extensive structural studies have demonstrated that $\mathrm{Cu}^{2+}, \mathrm{Zn}^{2+}$ and $\mathrm{Fe}^{3+}$ ions can form coordinate bonds between $A \beta$ peptides and increase their aggregation propensity ${ }^{140}$. Interestingly, $A \beta$ fibers assembled in the presence of sub-stoichiometric concentration of $\mathrm{Cu}^{2+}$ possess increased cytotoxicity in comparison to aggregates generated in the absence of $\mathrm{Cu}^{2+}$ ions ${ }^{142}$. High cytotoxicity of $\mathrm{Cu}-\mathrm{A} \beta$ complex is likely due to its strong potential to generate ROS ${ }^{143}$. Involvement of other metals in AD, such as aluminum, has been debated ${ }^{144}$.

Metals can serve as cofactors for various proteins to complement their functions. For example, Fe is incorporated into the protoporphyrin ring to form heme. Heme is a prosthetic group of hemoglobin that is responsible for the distribution of oxygen throughout the body. But it is also present in every metabolically active cell where it is an essential component of complex II, III and IV of mitochondrial electron transfer chain ${ }^{145}$. Heme metabolism, cell death response and $\mathrm{AD}$ are tightly interconnected. Proteins that are linked to heme metabolism have been reported to be dysregulated in $\mathrm{AD}^{146,147}$. As discussed in the previous section, microbleeds provoked by extended CAA can cause heme leakage from blood vessels into brain. High concentration of free heme is toxic and leads to lipid peroxidation and increased production of ROS ${ }^{148,149}$. Moreover, amyloid- $\beta$ can form a complex with heme that possess strong peroxidase and superoxide activity that can contribute largely to cytotoxicity during $\mathrm{AD}^{150-152}$.

\subsubsection{Other processes}

$\mathrm{AD}$ pathogenesis is not restricted to alterations in the biological pathways mentioned above. Processes such as protein glycation ${ }^{153}$, autophagy ${ }^{154}$, synaptic transmission ${ }^{155}$ and others are also affected in AD. Despite our knowledge of the pathways involved in the disease, the relationships between them remain enigmatic. Further research efforts in the field will be required to identify the missing connections between affected processes and pathways in order to better decipher the progression of Alzheimer's disease. 


\subsubsection{Therapeutic strategies}

Since few decades, research community and pharmaceutical industry have been in search of effective anti-AD therapeutics. Several strategies have been followed along this path.

\subsubsection{Acetylcholine esterase inhibitors and NMDA antagonists}

The levels of neurotransmitter acetylcholine are significantly reduced in the brains of AD patients. According to cholinergic hypothesis, synapses that use acetylcholine as a neurotransmitter degenerate first in $\mathrm{AD}^{156}$. For this reason, inhibition of acetylcholine esterase, an enzyme that is responsible for acetylcholine degradation in the synapse, seemed to a promising therapeutic strategy. The drugs targeting this enzyme were the first to get FDA approval for treatment of AD. Nevertheless, administration of acetylcholine esterase inhibitors has a very modest impact of AD symptoms and does not stop or slow down the progression of the disease ${ }^{157,158}$.

Glutamate is another neurotransmitter directly related to AD. Increased release of glutamate in $\mathrm{AD}$ was proposed to cause cell death due to excitotoxicity ${ }^{159}$. Memantine, the antagonist of NMDA receptor (ionotropic glutamate receptor), was developed to treat AD. Memantine was approved by FDA and EMA but was not effective in stopping the progression of the disease ${ }^{160}$. It also remains controversial whether memantine produces any significant symptomatic relief in AD patients ${ }^{161,162 .}$

\subsubsection{Therapeutic targeting of $A \beta$}

Discovery of the central role of $A \beta$ in $A D$ pathology made it an attractive therapeutic target. One potential route to reduce the levels of $A \beta$ is to interfere with its production by $\beta$ - and $\gamma$ secretases. The problem with such treatment is the fact that these proteases have other substrates apart from APP (e.g. Notch, voltage-gated sodium channels) ${ }^{163}$. Recently, $\gamma$ secretase inhibitor was withdrawn from stage III clinical trial for worsening cognitive parameters in treated AD patients potentially due to inhibition of other physiologically important functions of $\gamma$-secretase ${ }^{164}$. Other therapeutic strategies have aimed at generation of shorter, less toxic $A \beta$ peptides by altering $\gamma$-secretase activity. Nevertheless, the clinical trial utilizing this strategy was not successful ${ }^{165}$. Currently, few more selective $\beta$ - and $\gamma$ secretase inhibitors are in clinical trials. 
Other strategies are focusing on direct removal of $A \beta$ by immune system. To this end, several anti $A \beta$ vaccines have been developed. This strategy was successful in transgenic mice overexpressing APP166. However, $A \beta$ immunization in humans led to unwanted immunopathological effects and clinical trials utilizing this method had to be stopped ${ }^{167 .}$ Application of passive immunization by injection of humanized monoclonal anti-A $\beta$ antibodies is also being tested. One of the first drugs in this category, bapineuzumab, was successful in reducing perivascular accumulation of amyloid in mouse model of AD ${ }^{168}$. In clinical trials, bapineuzumab engaged $A \beta$ in patient's brain, however, it failed to evoke any beneficial response ${ }^{169}$. Small molecule drugs that neutralize toxic A $\beta$ oligomers are also under development. One such drug, ELND005 (scyllo-inositol), has been already tested in clinical trials but did not demonstrate the desired outcome with regard to symptoms and progression of the disease ${ }^{170}$. As mentioned before, $A \beta$ aggregation is positively regulated by metal ions that can form coordinate bonds between $A \beta$ monomers consequently speeding up the nucleation of aggregates. Use of molecules chelating such metal ions provided promising results in mouse models of $\mathrm{AD}^{171}$. Clinical trials for this type of therapeutics are ongoing and will assess whether this strategy can lead to beneficial outcome in patients.

\subsubsection{Anti-tau therapeutics}

The interest in tau as a therapeutic target for AD has been growing over the last decade ${ }^{172}$. The first group of anti-tau therapeutics revolve around methylene blue, a small molecule capable of inhibiting tau aggregation ${ }^{173}$. Methylene blue was the first fully synthetic drug applied in medicine and was originally used to combat malaria ${ }^{174}$. Ever since, its analogs have been frequently used in clinics as a placebo control before its positive psychotropic effects were discovered ${ }^{175}$. Stage II clinical trials for Rember TM (commercial name of methylene blue, TauRx Therapeutics) delivered somewhat promising results ${ }^{176}$. However, due to the poor pharmacokinetics of the drug, the trial was discontinued. Stabilized analog of methylene blue (LMTM) is now being tested in stage III clinical trial for the treatment of AD and frontotemporal dementia. Clinical trials for several tau vaccines and anti-tau monoclonal antibodies are currently ongoing. However, no information is available regarding their efficiency so far. Since hyperphosphorylation of tau was shown to increase its aggregation potential, therapeutic strategies targeting tau kinases have also been considered ${ }^{177}$. One caveat of such approach is the high off-target effect of kinase inhibition as most of the tau 
kinases have hundreds of other substrates important for various physiological processes. Few small molecule drugs that stabilize microtubules and counteract tau pathology are now being tested for the treatment of $\mathrm{AD}^{178,179}$.

\subsubsection{Other therapeutic strategies and perspectives}

Increasing relevance of $\mathrm{APOE}$ in $\mathrm{AD}$ progression also attracted attention to this protein as a putative therapeutic target. Specific targeting of APOE4 isoform by disruption of its domain interaction is being studied now as a potential path for therapeutic intervention ${ }^{180}$. In line with APOE function in AD, other therapeutics that revolve around cholesterol metabolism have been designed ${ }^{181,182}$. Several new strategies focus on inflammation and oxidative stress as primary processes involved in $\mathrm{AD}^{183,184}$.

Over the last three decades, active preclinical research brought almost 200 different therapeutic candidates into clinical studies. Despite being promising in the preclinical phase, none of them were effective in stopping the progression of $\mathrm{AD}$ in patients. There are several reasons that could explain such a poor outcome. Low penetration of BBB, especially by antibody-based therapeutics, can be the cause for reduced therapeutic efficiency. Most clinical studies involved AD patients at moderate or severe symptomatic stages of AD. The failure to stop the disease in such individuals might be explained by the fact that the treatment was administered simply too late after the full onset of the disease. Successful results in preclinical development and the failure to reproduce the outcome in patients was often blamed on imperfect modeling of $\mathrm{AD}$ in animals ${ }^{185}$. But perhaps the most concerning reason could be the choice of wrong targets. Although the roles of $A \beta$, tau and APOE in AD have been studied extensively, we still do not fully understand the mechanisms behind the origin of the disease and the exact contribution of these proteins to it. Clearly, new strategies that will consider the lessons from the failed trials are required for successful development of effective $\mathrm{AD}$ therapeutics in the future.

\subsection{Animal models of Alzheimer's disease}

Animal models contribute profoundly to uncovering new concepts and mechanisms in fundamental biology and the understanding of human diseases. Due to the complexity of AD, the establishment of adequate models that can mimic all aspects of the disease is very Introduction $\mid 26$ 
challenging. Current animal models of AD can be classified into three major categories based on the way AD pathology is invoked: spontaneous, interventional and genetic models.

\subsubsection{Spontaneous and interventional models}

Spontaneous models of AD are based on detection and observation of AD-like pathology in aging animals. Some non-human primates develop certain features of AD with aging. For instance, baboons develop NFT pathology but do not exhibit A $\beta$ plaques ${ }^{186}$. In contrast, macaques can form plaques but demonstrate no signs of tau tangles ${ }^{187}$. Other mammalian species such as $\operatorname{dog} s^{188}$, cats ${ }^{189}$, bears ${ }^{190}$, goats and sheep ${ }^{191}$ exhibit age-dependent accumulation of $A \beta$ or neurofibrillary tangles. However, unpredictable development of the disease onset and thus maintenance of large animal colonies makes the use of spontaneous models rather impractical.

Interventional models rely on administration of chemical substances or formation of lesions in specific brain regions ${ }^{192}$. The first generation of interventional models was developed based on the cholinergic hypothesis of AD progression. Degeneration of cholinergic neurons in nucleus basalis (Meynert, Ch4) is one of the early events in AD progression ${ }^{156}$ and thus targeting these neurons seemed to be a viable option to model the disease. Administration of scopolamine, an antagonist of muscarinic receptor, in rodents invokes amnesia and impacts various cognitive functions typically affected in $\mathrm{AD}^{193,194}$. Induction of lesions in cholinergic centers either mechanically or electrochemically has led to generation of another subset of interventional models that recapitulated similar phenotypes ${ }^{195,196}$. Targeted degeneration of hippocampus, striatum or cortical regions allowed to partially mimic AD symptoms related to memory loss ${ }^{197-199}$. Some other approaches focused on induction of specific aspects of AD pathology such as inflammation or impaired glucose metabolism ${ }^{200,201}$. Although all these models mimic some symptoms related to $\mathrm{AD}$, they do not develop plaques or tangles and in most of the cases lack further progressive amelioration of cognitive functions after the lesion is established or the effect of a drug is gone.

According to the amyloid cascade hypothesis, accumulation of insoluble $A \beta$ peptides is the main driver of AD pathogenesis. In line with this theory, interventional animal models were developed based on intracerebral or intracerebroventricular infusion of $A \beta$ peptides. These models recapitulated several key phenotypes observed in AD such as memory and learning 
impairments and behavioral alterations ${ }^{202,203}$. Advantage of $A \beta$ infusion models lays in the direct effect of $A \beta$ on the brain. Additionally, repetitive administration of $A \beta$ allows to study progressive aggravation of AD-related pathology. $A \beta$ infusion models are also devoid of artifacts related to overexpression of full length APP in genetic models of $\mathrm{AD}^{204}$. On the other hand, these models do not consider aging which is an important factor in AD development in human. Moreover, the concentration of $A \beta$ used to invoke pathology is typically higher by several orders of magnitude in comparison to those occurring in the brain of patients 205 . Finally, infusion of $A \beta$ is an invasive procedure which causes brain injury and may lead to unwanted side effects (e.g. inflammation) and due to its complexity can reduce reproducibility of experimental findings.

\subsubsection{Genetic models}

\subsubsection{General considerations}

With the rapid development of gene transfer technologies, establishing of genetic animal models for various diseases have become a routine. 139 different genetic mouse models of AD have been developed over the last three decades (complete list of genetic mouse models is available on Alzforum: http://www.alzforum.org/research-models). These models can be differentiated by three key aspects: the transgenes used to simulate the disease, promoter controlling the expression of these transgenes and the method by which they were incorporated into genome.

Two common strategies are used to introduce transgenes. The first strategy is based on the pronuclear injection of a transgene (including exogenous promoter) into a zygote and subsequent implantation of embryo into pseudopregnant female mouse. In this way, the transgene is inserted randomly into the mouse genome and overexpressed under control of introduced promoter. In the second approach, an endogenous mouse gene is modified by homologues recombination with a DNA vector injected into embryonic stem cells. Modified

cells are then injected into blastocysts and mice homozygous for the introduced mutation are selected. As a result, the targeted gene will be expressed under control of the endogenous promoter at physiological level (knock-in mice).

Although the knock-in approach enables "clean" insertion of a transgene into its native locus, historically most of the transgenes in mouse models of $\mathrm{AD}$ were introduced by pronuclear 
injection due to relatively simpler procedures. The use of an adequate promoter in such system is thus crucial to ensure expression of the gene at a desired place and at an adequate level. Typically, promoters of platelet-derived growth factor $\beta$-chain (PDGF- $\beta$ ), the thymocyte differentiation antigen 1 (Thy1) or prion protein (PrP) are utilized for overexpression of FAD genes $^{55}$. PDGF- $\beta$ and Thy1 promoters restrict expression of transgenes to neurons, while $\operatorname{PrP}$ is expressed in a broader range of neural cells. Mouse models utilizing ApoE4 most commonly express the gene under NSE or GFAP promoters specific to glia cells.

Table 1-1. The most common promoters in mouse models of AD.

The relative level of expression is indicated as,+++ , and +++ . E\# indicates embryonic day number; P\# indicates postnatal day number. Adapted from Esquerda-Canals et al., $2017^{55}$.

\begin{tabular}{|c|c|c|c|}
\hline \multicolumn{2}{|c|}{ Promoter Spatial pattern } & Level & Beginning \\
\hline \multicolumn{4}{|c|}{ Highly used for FAD-associated transgenes } \\
\hline \multirow[t]{2}{*}{ PDGF- $\beta$} & Neuron-specific: dendrites, axons, and terminals. & + & E15 \\
\hline & $\begin{array}{l}\text { Brain, dorsal horn of the spinal cord, and posterior } \\
\text { pituitary. }\end{array}$ & & \\
\hline \multirow[t]{3}{*}{ Thy-1 } & Neuron-specific. & ++ & P6-10 \\
\hline & Long-projection neurons from both the CNS and PNS. & & \\
\hline & Human Thy1 is also expressed in neuroglia. & & \\
\hline $\operatorname{PrP}$ & $\begin{array}{l}\text { Neurons, astrocytes, oligodendrocytes, and microglia. } \\
\text { Also expressed in extraneural tissues. }\end{array}$ & +++ & E12.5 \\
\hline \multicolumn{4}{|c|}{ Highly used for apoE transgenes } \\
\hline NSE & Mature neurons and neuroendocrine cells. & ++ & \\
\hline GFAP & Astrocytes. & +++ & E12.5-13.5 \\
\hline
\end{tabular}

\subsubsection{APP overexpression models}

Most of the genetic models of AD revolve around the genes involved in FAD (APP, PSEN1 and PSEN2). Rodent $\mathrm{A} \beta$ differs from the human variant by three amino acids. This fact potentially explains why rodents do not develop plaque pathology in the first place ${ }^{206}$. Initial attempts to overexpress human APP in mice resulted in the moderate accumulation of extracellular A $\beta$ deposits 207,208 . However, no significant behavioral or cognitive alterations could be detected.

To overcome this problem, the human APP gene with Indiana mutation was introduced into mice under control of PDGF promoter (PDAPP mice) ${ }^{209}$. These mice exhibited considerable number of $A \beta$ plaques at 9 months of age and manifested synaptic loss and gliosis. Successful 
generation of PDAPP mice encouraged the development of further genetic models based on other FAD-related mutations in APP gene. For example, Tg2576 and APP23 mouse models harbored APP with Swedish mutation 210,211 . These models differ by the isoform of expressed APP. The 751 amino acid long isoform of APP (APP751) which is more prone to cleavage by $\gamma$ secretase $^{212}$ was introduced into APP23 mice, while shorter APP695 gene was expressed in Tg2576 model. Interestingly, the difference in the length of APP leads to the later onset of A $\beta$ plaque formation in Tg2576 mice (11 months versus 6 months in APP23 model). Mice models overexpressing APP with combination of several FAD-related mutations demonstrated even more severe phenotypes and earlier onset of $A \beta$ pathology $213-215$.

\subsubsection{APP/PSEN double transgenic models}

Although it might seem more intuitive that mutated APP is the main contributor to inherited forms of $\mathrm{AD}$, most of FAD cases are caused by mutations in PSEN1216. Substitution of methionine at position 146 to valine or leucine is the most common mutation of PSEN1 in FAD and has been shown to alter activity of $\gamma$-secretase resulting in increased production of highly toxic $A \beta_{1-42}$ species ${ }^{217}$. The first genetic models involving $\gamma$-secretase were based on the knocked-in PSEN1 harboring mutation at M146. As expected, these mice demonstrated elevated $A \beta_{1-42}$ production and increased susceptibility of hippocampal neurons to excitotoxic necrosis ${ }^{218}$. Nevertheless, models based on mutations in PSEN1 or 2 alone did not display most of the characteristic features of AD pathology 219,220 .

Combination of mutated APP and PSEN1 transgenes was a logical next step in modeling of AD. Generally, such bigenic models demonstrate faster and more severe progression of the disease. Crossing of Tg2576 line with mouse overexpressing mutated PSEN1 gave origin to

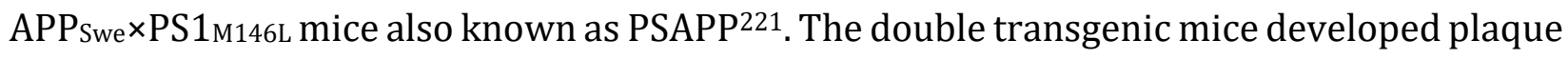
pathology faster than their monogenic Tg2576 counterparts. Interestingly, these transgenic mice exhibited memory deficits prior to extracellular $A \beta$ deposition supporting the role of soluble/oligomeric $\mathrm{A} \beta$ as primary toxic agent during $\mathrm{AD}$. Another step forward in genetic modeling of $\mathrm{AD}$ was the generation of double APPSwe $/$ PSEN1P264L knock-in mice $(2 \times \mathrm{KI})^{222}$. The age of onset for $\mathrm{A} \beta$ plaque pathology in $2 \times \mathrm{KI}$ line was the same as in mice overexpressing identical transgenes. However, the amyloid load of brain in $2 \times \mathrm{KI}$ mice was growing linearly over the course of aging in comparison to exponential growth in the corresponding overexpression model.

Introduction | 30 
To maximize the AD-like pathology in mice, the group of Robert Vassar generated a so-called $5 \times$ FAD line, double transgenic APP/PSEN1 mice that incorporated five different mutations occurring in FAD (Swe, Lon and Fl mutations of APP and M146L and L286V mutations in PSEN1) ${ }^{223}$. These mice display extremely aggressive pathology characterized by very early accumulation of intraneuronal $A \beta$ deposits (1.5 months) and extracellular $A \beta$ plaque formation ( 2 months). $5 \times$ FAD mice also exhibit early gliosis, loss of synaptic proteins and neuronal death. Significant memory deficits could be also detected in 5×FAD. Although $5 \times$ FAD mice display very apparent AD-like pathology, this model has been criticized for the extreme overexpression of transgenes and very early onset of the disease which makes it less comparable to $\mathrm{AD}$ in human 224 .

\subsubsection{Mouse models utilizing non-FAD transgenes}

Since FAD accounts for only about $1 \%$ of all AD cases, genetic models aiming to mimic SAD were developed. The strongest genetic risk factor for sporadic forms of AD is APOE $\varepsilon 4$ allele ${ }^{59}$. The role of human APOE was studied by overexpression of $\varepsilon 3$ and $\varepsilon 4$ alleles in mice devoid of endogenous $A P O E$ gene $^{225}$. Remarkably, mice expressing human APOE $\varepsilon 4$ demonstrate impairments in learning and exploratory behavior that are dependent on age and gender. Female mice are more affected by $\varepsilon 4$ allele than age-matched males which correlates with the higher prevalence of AD among women in human. More recent models combining mutations in FAD genes with expression of human APOE variants demonstrated an important role of APOE in A $\beta$ clearance, regulation of gliosis and inflammation ${ }^{226,227}$.

Additionally, the role of other human genes in AD was probed using mouse models. For example, concomitant expression of mutated APP with a double-negative receptor for advanced glycation products (RAGE) construct diminished neuropathologic changes, indicating the role of RAGE as an important cofactor for $A \beta$ pathology 228 . Knockout of TREM2 in $5 \times \mathrm{FAD}$ mice has been shown to augment $A \beta$ aggregation due to inefficient microglia response $^{229}$. A mouse model based on the knock-in of human $\beta$-secretase demonstrates an increase in APP cleavage and reiterates some features of AD pathology ${ }^{230}$. Crossing already existing APP models with mice deficient in NOS2 and PDGFR allowed to uncover the role of such processes as oxidative stress and neurovascular dysfunction in $\mathrm{AD}^{231,232}$. 


\subsubsection{Triple-transgenic mouse model of $A D$}

The monogenic or bigenic mouse models based on human APP and/or PSEN could successfully recapitulate many aspects of $A \beta$-related pathology. However, none of them displayed another characteristic feature of $\mathrm{AD}$, namely tau tangles. To address this problem Oddo and colleagues introduced triple-transgenic mouse model $(3 \times \mathrm{Tg}-\mathrm{AD})$ that harbors knocked-in PSENM146v, overexpressed APPswe and mutated variant of human tau (MAPTP301L, $4 \mathrm{R}$ isoform $)^{233}$. The mutation in tau at position P301 was initially identified in frontotemporal dementia ${ }^{234}$. It reduces affinity of tau to microtubules and increases its aggregation propensity.

$A \beta$ plaques
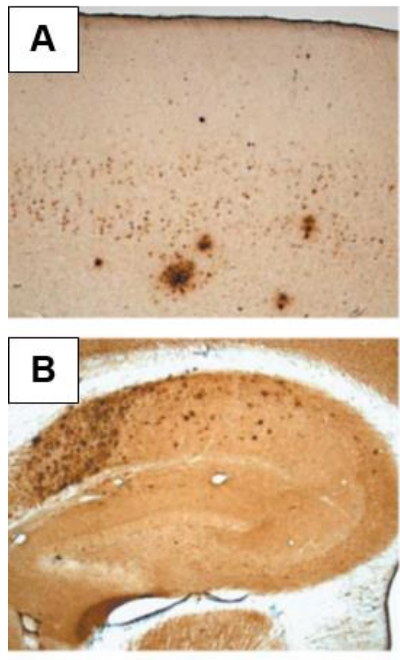

Phospho-tau
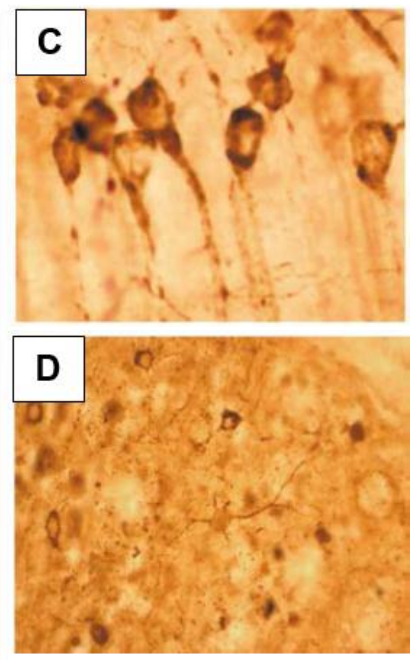

Figure 1-7. Neuropathology in $3 \times$ Tg-AD mouse model

Low-magnification view of neocortex (A) and hippocampus (B) from 18-month-old $3 \times \mathrm{Tg}$-AD mouse following staining with $\mathrm{A} \beta 42$-specific antibody.

(C) High-magnification view showing immunopositive neurons following staining with antibody PHF1, which detects phosphorylated residues S396 and S404. (D) High-magnification view of the subiculum showing taureactive dystrophic neurites surrounding globular structures, which are thioflavin-positive $A \beta$ plaques.

Adapted from Oddo et al., $2003^{233}$.

$3 \times \mathrm{Tg}-\mathrm{AD}$ mice progressively develop $\mathrm{A} \beta$ plaques and tau tangles. Intracellular deposits of $\mathrm{A} \beta$ can be observed as early as at 6 months of age with extracellular plaques becoming prominent in 12-month-old animals. In contrast, the tau pathology takes longer time to develop. NFTs can be first detected between 12 and 15 months ${ }^{235}$. This sequence of events tightly correlates with the development of the disease in human, where plaque accumulation was shown to precede tau pathology ${ }^{39,48}$. Apart from neuroanatomical signs of AD, the $3 \times \mathrm{Tg}$ - 
AD model develops characteristic memory deficits ${ }^{236}$. Synaptic function is inhibited at 6 months as determined by reduction in long-term potentiation (LTP) ${ }^{233}$. Likewise, extensive gliosis can be observed at this age ${ }^{237}$. Close resemblance to human pathology, relatively mild overexpression of inserted transgenes and gradual progression of the disease make $3 \times \mathrm{Tg}-\mathrm{AD}$ mice one of the most popular genetic mouse models to study AD.

\subsection{High-throughput profiling of gene and protein expression in AD}

Changes in gene expression can indicate underlying physiological and pathological alterations during disease progression. The knowledge of such changes can help to decipher molecular mechanisms governing pathogenesis. Furthermore, systematic profiling of gene expression in tissues and biological fluids (either from humans or animal models) can identify early biomarkers of pathological state so much needed for AD diagnosis.

The high-throughput approaches or so-called "-omics" techniques allow for simultaneous detection and quantitative measurement of biological molecules. Advances in molecular genomics made it possible to map human genome and helped to identify mutations leading to FAD as well as alleles and single nucleotide polymorphisms (SNPs) that increase the risk of developing SAD. Transcriptomics and proteomics approaches enable estimation of gene expression on mRNA and protein level, respectively. Transcriptome and proteome mapping can provide valuable insights into the mechanisms of diseases since up- or downregulation of protein expression can serve as an indication of pathological process.

\subsubsection{Microarray approaches}

Levels of mRNA can serve as proxies to protein expression. Traditionally, quantification of specific RNA in biological sample was performed using Northern blotting or quantitative real-time PCR. However, the use of these methods is limited to quantification of single (or in some cases few) RNA sequence(s) at the time. Development of microarray techniques in the 1990s enabled simultaneous high-throughput quantification of transcripts ${ }^{238}$. In classical microarray experiments, mRNA is first transcribed to complementary DNA (cDNA) which is then hybridized with the array of single-strand DNA probes specific to individual genes. Successful hybridization leads to an increase in the readout value (typically fluorometric or 
luminometric) for specific gene, hence quantitative information on RNA levels can be obtained $^{239}$.

Studies utilizing cDNA microarray approaches helped to expand our knowledge of transcripts regulated in AD. Several studies revealed that the expression of genes involved in energy metabolism, oxidative stress, DNA damage, senescence and synaptic function is primarily dysregulated in $\mathrm{AD}$ brains 240,241 . Another report demonstrated that the levels of diabetes-related mRNAs differed significantly between the brains of healthy and AD individuals ${ }^{242}$. In the same study, akin results were observed for the transcriptome obtained from hippocampus of $3 \times \mathrm{Tg}$-AD model. Microarray profiling of mRNA isolated from microglia revealed similar network of genes to be regulated upon microglia activation in different neurodegenerative disorders (AD and ALS) as well as normal aging ${ }^{243}$. Another transcriptomic study on acutely isolated glia revealed that both astrocytes and microglia acquired a proinflammatory transcriptome profile in APPSwe/PSEN1de9 double transgenic, but not in control mice. Interestingly, upregulation of immune response genes in astrocytes was stronger than in microglia and was accompanied by reduced expression of neuronal support genes ${ }^{244}$.

\subsubsection{RNA sequencing}

Nowadays, transcriptomics is transitioning from hybridization microarrays to the next generation sequencing methods (RNA-seq). RNA-seq could overcome some of the major limitations of microarray approach such as cross-hybridization artifacts, imprecise quantification of low and high abundant transcripts and the requirement for prior knowledge of the tested sequence. RNA-seq analysis also covers all species of RNA, including non-coding RNAs. Additionally, RNA-seq enables identification of differently spliced transcripts. Another advantage of contemporary RNA-seq techniques is high sensitivity. Total RNA isolated from a single cell is typically enough to identify thousands of different RNA species ${ }^{245}$. These single-cell sequencing techniques are thus a powerful method to uncover changes in expression profile with regard to individual cell type. This aspect of RNA-seq technology is particularly useful in AD research since it allows to determine an individual contribution of neurons, astrocytes, microglia and other cell types to the progression of the disease. 
Single cell RNA-seq analysis of microglia derived from inducible mouse model of severe neurodegeneration demonstrated a rapid remodeling of microglia transcriptome after induction of neurodegeneration ${ }^{246}$. The same study also reported a wide heterogeneity in microglia responding to neurodegenerative stimuli and time-dependent cell reprogramming occurring with the disease progression. Further RNA-seq-based studies led to identification of microglia subtype that occurs specifically in $\mathrm{AD}^{247}$. These cells exhibit particularly strong expression of APOE and TREM2 and protect brain from neurodegeneration by phagocytic removal of $A \beta$ and other toxic products. A recent study applying mathematical inference model to publicly available mRNA-seq data, revealed that the decay of mRNAs encoding synaptic proteins occurs faster in the brain of AD patients than in age-matched controls ${ }^{248}$. The authors speculate that the reason for such difference is reduced expression of mRNA stabilizing protein RBFOX1 in individuals affected by AD.

The role of non-coding RNAs has been also examined using RNA-seq. The group of Shumpei Niida has developed a pipeline for identification of miRNAs from publicly available databases and have already shown a significant difference in the levels of 27 miRNAs in the blood of AD patients ${ }^{249}$. This approach is thus promising for the identification of miRNAs as biomarkers of AD. Furthermore, the function of another class of non-coding RNAs termed long intergenic non-coding (lincRNAs) in neurodegeneration is emerging 250,251 . Interestingly, a recent study demonstrated that lincRNAs undergo even larger changes in AD than the protein coding transcriptome 252 . Although the regulation of lincRNAs seems to play a role in AD development, more studies are required to understand the function of such RNAs in the brain and their relation to the disease.

\subsubsection{Mass-spectrometry based proteomics}

Quantitative measurement of mRNA by microarray or RNA-seq techniques has expanded our knowledge of transcripts involved in AD. Nevertheless, most of the biological processes are manifested through proteins. mRNA levels do not always linearly correlate with expression

of corresponding protein ${ }^{253}$. To overcome this bias, various techniques for protein identification and quantification were developed. For instance, protein levels can be assessed by application of antibodies or ligands that specifically bind the protein of interest. Although specific, these methods are limited to identification of a single protein. Additionally, 
immunological techniques allow only for identification of proteins that have been already discovered and for which specific antibodies or ligands are available.

Alternatively, protein composition of a biological sample can be analyzed by $\mathrm{N}$-terminal sequencing which was developed in 1949 by Pehr Edman ${ }^{254}$. This method does not depend on the prior knowledge of protein or availability of specific antibodies and is well suited for identification of novel proteins. Nevertheless, use of Edman sequencing in high-throughput protein quantification is restricted by several technical limitations and insufficient accuracy of quantitative measurement 255 .

The world of proteomics was revolutionized by development of quantitative massspectrometry (MS). In this method, proteins are typically digested enzymatically to obtain smaller peptide fragments. These individual peptides can be then identified and quantified based on their mass to charge ratio $(\mathrm{m} / \mathrm{z})$. In the next step, the data obtained for each peptide can be combined and processed to infer the relative quantity of proteins in the sample. Atypical mass spectrometer consists of an ionizer (converts injected peptides into ions), a mass analyzer (selects ions based on their $\mathrm{m} / \mathrm{z}$ ratio) and a detector (determine quantity of selected ion). Current tandem mass spectrometry techniques enable us to quantify thousands of proteins from complex biological samples on the time scale of minutes or hours. Explorative proteomic studies typically apply a so-called "shotgun" approach where data are acquired in data-dependent acquisition. In this case, most abundant peptides will be selected for fragmentation and further quantification as they appear in mass-analyzer. As a drawback, the low abundant peptides will elude the analysis. Nevertheless, improvement in the MS instrumentation and peptide separation techniques allowed to considerably increase the dynamic range of detection in shotgun proteomics ${ }^{256}$. For better quantification of specific peptides/proteins, data-independent acquisition methods such as multiple reaction monitoring (MRM) or SWATH were developed. However, the higher accuracy of the dataindependent acquisition approaches comes with a trade-off of lower sensitivity and coverage ${ }^{257}$.

Mass spectrometry has been successfully applied to elucidate changes of brain proteome in mouse models of $\mathrm{AD}$. A dysregulation of mitochondrial proteome was reported in $3 \times \mathrm{Tg}-\mathrm{AD}$ mice ${ }^{258,259}$. Another proteomic study in $3 \times \mathrm{Tg}$-AD model strengthened the hypothesis that 
oxidative phosphorylation is impaired in $\mathrm{AD}$ revealing upregulation of numerous proteins involved in glucose catabolism ${ }^{260}$. Recent proteomic study in monogenic APP and double transgenic APP/PSEN1 mice demonstrated dysregulation of protein networks related to synapse, mitochondria and cytoskeleton ${ }^{261}$. Interestingly, the upregulation of endogenous mouse APOE4 in cortex and hippocampus of transgenic mice was detected in this report as well.

Initial investigations of human brain proteome unraveled changes in similar pathways as were described in mice ${ }^{262}$. To focus the analysis on specific subset of proteins, many studies utilized subcellular fractionation prior to MS measurement. For example, isolation of nerve terminals from postmortem brains allowed to perform comprehensive proteomic analysis of synapses while reducing the background of high abundant proteins of neuronal soma and non-neuronal cells ${ }^{263}$. The follow-up study from the same group applied MRM to quantify the differences in most prominent synaptic hits with higher precision ${ }^{264}$. This analysis demonstrated downregulated expression of several synaptic proteins as well as strong elevation of peroxiredoxin-1, a protein directly involved in response to oxidative stress. A similar approach was applied to postsynaptic density (PSD) proteins, which were first enriched by subcellular fractionation from brains of $\mathrm{AD}$ and age-matched healthy individuals ${ }^{265}$. Interestingly, researchers found that among the core PSD proteins only one (IRSp53) was significantly diminished in AD brains suggesting that the reduction of specific proteins rather than extensive downregulation of entire synaptic proteome can inhibit neurotransmission in AD. Isolation of SDS-insoluble fraction of brain proteins from postmortem brains of AD patients revealed accumulated components of mRNA processing machinery in $\mathrm{A} \beta$ plaques proposing the role for dysregulated alternative splicing in $\mathrm{AD}^{266}$.

MS-based proteomics has been used on another front of AD research, namely the identification of biomarkers. Classical CSF biomarkers of AD include increased tau and phospho-tau as well as reduced soluble $A \beta_{1-42^{47}}$. These markers can be detected by MS techniques to determine the disease with about $85-95 \%$ specificity ${ }^{267}$. Nevertheless, this impressive accuracy holds true only for fully developed symptomatic AD. Therefore, explorative MS-studies have been conducted to identify presymptomatic biomarkers that can be detected before the onset of dementia. Dysregulated proteins identified in CSF of FAD patients before manifestation of symptoms were related predominantly to synapse or 
inflammatory response 268 . Isolation of exosomes from CSF and downstream proteomic characterization was proposed as a new approach for identification of AD biomarkers as it might help to enrich disease-relevant proteins secreted by damaged cells ${ }^{269}$. Identification of plasma-based biomarkers has also demonstrated some progress over the last years. Thanks to MS-based proteomics, positive correlation between levels of clusterin (APOJ) in plasma and severity of AD was found ${ }^{270}$. Recent systematic study of plasma biomarkers reported that APP $/ A \beta_{1-42}$ and $A \beta_{1-40} / A \beta_{1-42}$ ratios determined by immunoprecipitation coupled with mass spectrometry correlate strongly with amyloid deposits in the brain assessed by PET scanning and thus can serve as a concomitant diagnostic method ${ }^{46}$.

\subsubsection{Assessment of posttranslational modifications}

The activity of biological function can be extrapolated from the expression levels of proteins involved in it. However, this is rather a crude simplification. For instance, such an extrapolation does not consider posttranslational modifications (PTMs) that can significantly alter activity of the protein. Yet PTMs such as phosphorylation, ubiquitination, oxidation, acetylation, $\mathrm{O}$ - and N-glycosylation, glycation and others are known to play important roles in $\mathrm{AD}^{32,153,271,272}$.

Protein phosphorylation is one of the most abundant and well-studied PTMs. A phosphate group can be placed on serine, threonine or tyrosine residues by protein kinases. Phosphorylation is a transient modification and can be reversed by protein phosphatases. This way, protein phosphorylation serves as an activity switch turning proteins on or off. The best example of such transient activation are kinases and phosphatases whose activity depends on the phosphorylation status of specific phoshposites ${ }^{273}$. Protein phosphorylation is thus considered to be a key process governing signal transduction in cells.

Systematic uncovering of protein phosphorylation became possible thanks to the improvements in MS instrumentation and post-acquisition analysis ${ }^{274}$. Identification of phosphopeptides in shotgun proteomics is complicated because of their relatively low abundance and consequent masking by more copious peptides. Therefore, methods to enrich phosphopeptides from original biological sample have been developed. The two most used methods in the field are immobilized metal affinity chromatography (IMAC) and metal oxide affinity chromatography (MOAC) ${ }^{274}$. Both methods rely on the interaction of the negatively 
charged phosphate group with metal cations or metal oxide matrixes. Different chromatography techniques (e.g. strong cation exchange) can be used for additional prefractionation of phosphopeptides. Enriched phosphopeptides can be then quantified by mass spectrometry using stable isotope labeling or label-free approaches.

As discussed above, hyperphosphorylation of tau has devastating consequences on the health of a neuron during AD. However, AD-related changes in protein phosphorylation are not restricted to tau. The MS-based phosphoproteomic analysis of postmortem human brains revealed strong differences in phosphorylation of various kinases and small heat shock proteins between healthy and AD individuals in addition to hyperphosphorylation of microtubule-associated proteins ${ }^{275}$. Another study published the same year demonstrated dysregulated phosphorylation of proteins involved in general metabolism in $\mathrm{AD}^{276}$. Tagawa and colleagues compared AD brain phosphoproteome between several mouse models and human ${ }^{277}$. They identified a myristoylated alanine-rich C-kinase substrate (MARCS) to be strongly phosphorylated by protein kinase $\mathrm{C}$ (PKC) and $\mathrm{Ca}^{2+} /$ calmodulin-dependent kinase I and II (CaMKI/II) in AD across species. Furthermore, reversal of MARCS phosphorylation by inhibition of PKC and CaMKII rescued dendritic spine pathology in cultured neurons prepared from $5 \times$ FAD mice.

Although considerable technological progress in the field of phosphoproteomics has been achieved over the last decade, we still lack the knowledge of how the brain phosphoproteome changes over the course of $\mathrm{AD}$ progression. In particular, more systematic studies are required to untangle the signaling pathways affected in $\mathrm{AD}$ at the early stages of the disease.

\subsection{Aims of this thesis}

Protein expression levels can be indicative of physiological and pathological changes. Studies that applied microarray, RNA-seq and mass spectrometry analyses of postmortem brain tissue from $\mathrm{AD}$ patients have broadened our understanding of genes and proteins involved in AD. While such studies give important insights into pathology at molecular level at later stages, they provide limited information about the progressive alterations that occur over the development of the disorder. Particularly, early changes in protein expression preceding the onset of the disease would be missed. Understanding the sequence of the events 
happening in $\mathrm{AD}$ pathogenesis as well as initial molecular mechanisms driving its progression is crucial for the development of effective therapeutics and early diagnosis. For this reason, my $\mathrm{PhD}$ project focuses on the following three main objectives.

First, I wanted to understand how the expression of brain proteins changes over the course of $\mathrm{AD}$ progression. To this end, I applied quantitative mass-spectrometry to analyze the brain proteome of $3 \times \mathrm{Tg}-\mathrm{AD}$ mice at four specific time points that corresponded to distinct pathological stages of AD. In addition to expression changes, I examined alterations in protein phosphorylation as the major posttranslational modification affecting protein function. Using bioinformatics analysis of obtained quantitative data on protein expression and phosphorylation I could infer biological processes and molecular pathways that are affected at each specific stage of the disease.

The second aim of this study was to identify presymptomatic brain markers of AD. Aside from being potentially useful for early detection of $\mathrm{AD}$, these proteins could also be critically involved in the initial steps of the disorder culminating in clinical AD manifestation. Therefore, identifying these proteins can be an important step towards the design of early intervention strategies that can halt the initiation phase of AD. My analysis revealed a number of such presymptomatic markers including proteins with no previous record of involvement in AD. Additionally, I tested the expression of the most prominent presymptomatic markers in brain samples obtained from $\mathrm{AD}$ patients to confirm the relevance of the findings in $3 \times \mathrm{Tg}$ AD mouse model for the disease in human.

Finally, I aimed to take a closer look at the putative presymptomatic markers that have not been linked to $\mathrm{AD}$ before. In this study I took first steps to characterize one such novel marker, heme-binding protein-1 (Hebp1). I analyzed its potential biological function using various biochemical and cell culture techniques. These experiments revealed neuronal expression of Hebp1 in the brain, its association with mitochondria and role in apoptotic response making it a promising candidate involved in the development of Alzheimer's disease at its early stages. 


\section{Materials and Methods}

\subsection{Materials}

\subsubsection{Chemicals}

The standard chemicals used in this study were purchased from Merck (Darmstadt, Germany) and Sigma-Aldrich (Steinheim, Germany). All chemicals had at least analytical purity. Other chemicals are listed in Table 2-1.

Table 2-1. List of chemicals used in this study.

\begin{tabular}{|l|l|l|}
\hline Chemical & Source & $\begin{array}{l}\text { Catalog } \\
\text { number }\end{array}$ \\
\hline Albumin standard & Thermo Fisher Scientific & 23209 \\
\hline Protease and phosphatase inhibitor & Pierce & 88669 \\
\hline Acetonitrile & Thermo Fisher Scientific & 51101 \\
\hline $\begin{array}{l}\text { 2-[4-(2-hydroxyethyl)piperazin-1- } \\
\text { yl]ethanesulfonic acid (HEPES) }\end{array}$ & Gerbu Biotechnik & 1009.1000 \\
\hline $\begin{array}{l}\text { 2-Amino-2-hydroxymethyl-propane-1,3- } \\
\text { diol (Tris) }\end{array}$ & VWR International & 103156 X \\
\hline Paraformaldehyde (PFA) & Sigma & P6148 \\
\hline Rapigest & Waters, Milford, USA & 186002123 \\
\hline Titanium dioxide beads (TiO 2 ) & GL Sciences Inc. & $1400 \mathrm{~B} 500$ \\
\hline Dithiothreitol (DTT) & Thermo Fisher Scientific & 20290 \\
\hline Chloroacetamide (CAA) & Sigma & 22790 \\
\hline Phenylmethylsulfonylfluorid (PMSF) & AppliChem & 6367.3 \\
\hline Pepstatin & Peptide Institute & 4397 \\
\hline Ammonium bicarbonate & Sigma & A6141 \\
\hline Formic acid & Sigma & 56302 \\
\hline Acrylamide & AppliChem & A3626 \\
\hline Triton-X & Merck & K42092903 \\
\hline
\end{tabular}




\begin{tabular}{|l|l|l|}
\hline Nonidet P40 (NP-40) & AppliChem & A1694 \\
\hline Sodium dodecyl sulfate (SDS) & Serva & 20765.03 \\
\hline Coomassie Brilliant Blue G250 & AppliChem & A3480 \\
\hline
\end{tabular}

\subsubsection{Cell culture reagents}

Table 2-2. List of cell culture reagents used in this study.

\begin{tabular}{|l|l|l|}
\hline Chemical & Source & $\begin{array}{l}\text { Catalog } \\
\text { number }\end{array}$ \\
\hline $\begin{array}{l}\text { Dulbeccos Modified Eagles } \\
\text { Medium(DMEM) }\end{array}$ & Lonza & BE12-733F \\
\hline $\begin{array}{l}\text { Dulbeccos Modified Eagles } \\
\text { Eedium(DMEM)-F12 Medium }\end{array}$ & Sigma & D6421 \\
\hline Eagle's Minimum Essential Medium & Sigma & M2414 \\
\hline Opti-Mem I Reduced-serum Medium & Invitrogen & 11058021 \\
\hline Hank's Balanced Salt Solution (HBSS) & Lonza & BE10-547 \\
\hline Trypsin-EDTA & Lonza & $17-161 \mathrm{E}$ \\
\hline L-glutamine & Invitrogen & 25030123 \\
\hline L-cystein & Sigma & 30129 \\
\hline Papain suspension & Cell Systems & LS003126 \\
\hline DNaseI & Sigma & D5025 \\
\hline L-Alanyl-L-Glutamine & Millipore & K0302 \\
\hline Fetal Bovine Serum (FBS) & Pan Biotech & P30-8500 \\
\hline MEM-Vitamine & Sigma & K0373 \\
\hline Mito+Serum extender & Corning & 355006 \\
\hline D-glucose & AppliChem & A0883 \\
\hline B-27 supplement & Invitrogen & 17504044 \\
\hline Poly-D-lysine & Sigma & P-6407 \\
\hline
\end{tabular}




\begin{tabular}{|l|l|l|}
\hline 5-Fluoro-2'-deoxyuridine (FUDR) & Sigma & F0503 \\
\hline Puromycin & Sigma & P9620 \\
\hline Hemin & Sigma & 51289 \\
\hline tert-butyl-hydroperoxide & Sigma & 458139 \\
\hline staurosporine & Santa Cruz & sc-3510 \\
\hline Bovine serum albumin (BSA), fraction V & AppliChem & A1391 \\
\hline Lipofectamine 2000 & Life Technologies & 11668019 \\
\hline DMSO & Sigma & D-2650 \\
\hline
\end{tabular}

\subsubsection{Enzymes}

Table 2-3: List of enzymes used in this study.

\begin{tabular}{|c|c|c|}
\hline Enzyme & Source & Catalog number \\
\hline Sequencing grade modified trypsin & Promega & V5113 \\
\hline Lysyl endopeptidase, mass spectrometry grade & Wako Chemicals & $125-05061$ \\
\hline \multicolumn{3}{|l|}{ Restriction endonucleases } \\
\hline EcoRI-HF & NEB & R3101L \\
\hline AgeI-HF & NEB & R3552S \\
\hline NotI & NEB & R0189L \\
\hline XbaI & NEB & R0145L \\
\hline BsmBI & NEB & R0580L \\
\hline
\end{tabular}

\subsubsection{Kits}

Table 2-4: List of used kits.

\begin{tabular}{|l|l|l|}
\hline Kits & Source & Catalog number \\
\hline Pierce 660nm Protein Assay Reagent & Pierce & 22660 \\
\hline
\end{tabular}




\begin{tabular}{|l|l|l|}
\hline MultiTox-Glo & Promega & G9270 \\
\hline $\begin{array}{l}\text { CellEvent Caspase-3/7 Green Detection } \\
\text { Reagent }\end{array}$ & Sigma & C10723 \\
\hline GSH/GSSG-Glo Assay & Promega & V6611 \\
\hline $\begin{array}{l}\text { Mitochondrial Membrane Potential Assay Kit } \\
\text { (II) }\end{array}$ & Cell Signaling & $13296 \mathrm{~S}$ \\
\hline $\begin{array}{l}\text { ECL Select Western Blotting Detection } \\
\text { Reagent }\end{array}$ & GE Healthcare & RPN2235 \\
\hline GFP-trap & Chromotek & gtma-20 \\
\hline
\end{tabular}

\subsubsection{Antibodies}

Table 2-5: List of used antibodies.

WB (Western Blotting), IHC (Immunohistochemistry), ICC (Immunocytochemistry).

\begin{tabular}{|c|c|c|c|c|}
\hline Antibody & Specie & $\begin{array}{l}\text { Application } \\
\text { and dilution }\end{array}$ & Source & $\begin{array}{l}\text { Catalog } \\
\text { number }\end{array}$ \\
\hline Hebp1 & rabbit polyclonal & $\begin{array}{l}\text { WB }(1: 1000) \\
\text { IHC }(1: 100)\end{array}$ & Invitrogen & PA5-30609 \\
\hline Ctip2 & rat monoclonal & $\operatorname{IHC}(1: 100)$ & Abcam & ab18465 \\
\hline GFAP & $\begin{array}{l}\text { mouse } \\
\text { monoclonal }\end{array}$ & $\operatorname{IHC}(1: 500)$ & $\begin{array}{l}\text { Synaptic } \\
\text { systems }\end{array}$ & 173011 \\
\hline IBA1 & $\begin{array}{l}\text { guinea pig } \\
\text { polyclonal }\end{array}$ & $\operatorname{IHC}(1: 100)$ & $\begin{array}{l}\text { Synaptic } \\
\text { Systems }\end{array}$ & 134004 \\
\hline CA1 & rabbit polyclonal & WB $(1: 250)$ & $\begin{array}{l}\text { Novus } \\
\text { Biologicals }\end{array}$ & NBP1-8819 \\
\hline Glo1 & $\begin{array}{l}\text { mouse } \\
\text { monoclonal }\end{array}$ & WB (1:1000) & Genetex & GTX628890 \\
\hline Mic60 & $\begin{array}{l}\text { mouse } \\
\text { monoclonal }\end{array}$ & $\begin{array}{l}\text { WB }(1: 1000) \\
\text { ICC }(1: 100)\end{array}$ & Abcam & ab110329 \\
\hline$\alpha$-Tubulin & $\begin{array}{l}\text { mouse } \\
\text { monoclonal }\end{array}$ & WB (1:5000) & $\begin{array}{l}\text { Synaptic } \\
\text { Systems }\end{array}$ & 302211 \\
\hline$\beta$-actin & rabbit polyclonal & WB (1:5000) & $\begin{array}{l}\text { Synaptic } \\
\text { Systems }\end{array}$ & 251003 \\
\hline Amyloid beta $(A \beta)$ & $\begin{array}{l}\text { mouse } \\
\text { monoclonal }\end{array}$ & $\begin{array}{l}\text { WB (1:1000) } \\
\text { IHC (1:100) }\end{array}$ & Biolegend & 803004 \\
\hline Tau & rabbit polyclonal & WB (1:1000) & $\begin{array}{l}\text { Synaptic } \\
\text { Systems }\end{array}$ & 314002 \\
\hline $\begin{array}{l}\text { Phospho-tau } \\
\text { (Ser202;Thr205) }\end{array}$ & $\begin{array}{l}\text { mouse } \\
\text { monoclonal }\end{array}$ & WB $(1: 1000)$ & $\begin{array}{l}\text { Thermo } \\
\text { Fisher }\end{array}$ & MN1020B \\
\hline $\begin{array}{l}\text { Phospho-tau } \\
\text { (Ser400;Thr403;Ser404) }\end{array}$ & rabbit polyclonal & WB (1:1000) & $\begin{array}{l}\text { Cell } \\
\text { Signaling }\end{array}$ & $11837 \mathrm{~S}$ \\
\hline
\end{tabular}




\begin{tabular}{|l|l|l|l|l|}
\hline \multicolumn{5}{|l}{ Secondary antibodies for immunoblotting } \\
\hline rabbit IgG (HRP labeled) & goat polyclonal & WB (1:2000) & BioRad & 1706515 \\
\hline mouse IgG (HRP labeled ) & goat polyclonal & WB (1:2000) & BioRad & 1706516 \\
\hline $\begin{array}{l}\text { IRDye 800CW Anti- } \\
\text { Rabbit IgG }\end{array}$ & Goat polyclonal & WB (1:12,500) & Licor & $926-32211$ \\
\hline $\begin{array}{l}\text { IRDye 680CW Anti- } \\
\text { Mouse IgG }\end{array}$ & Goat polyclonal & WB (1:12,500) & Licor & $926-68070$ \\
\hline Secondary antibodies for immunostainings & & & \\
\hline $\begin{array}{l}\text { Alexa Fluor } 488 \text { anti- } \\
\text { mouse IgG }\end{array}$ & Goat polyclonal & IHC (1:750) & Invitrogen & A32723 \\
\hline $\begin{array}{l}\text { Alexa Fluor } 488 \text { anti- } \\
\text { rabbit IgG }\end{array}$ & Goat polyclonal & IHC (1:750) & Invitrogen & A-11034 \\
\hline $\begin{array}{l}\text { Alexa Fluor } 555 \text { anti- } \\
\text { mouse IgG }\end{array}$ & Goat polyclonal & IHC (1:750) & Invitrogen & A-21422 \\
\hline $\begin{array}{l}\text { Alexa Fluor } 546 \text { anti- } \\
\text { rabbit IgG }\end{array}$ & Goat polyclonal & IHC (1:750) & Invitrogen & A-11010 \\
\hline $\begin{array}{l}\text { Alexa Fluor } 555 \text { anti- } \\
\text { guinea pig IgG }\end{array}$ & Goat polyclonal & IHC (1:750) & Invitrogen & A-21435 \\
\hline $\begin{array}{l}\text { Alexa Fluor } 555 \text { anti-rat } \\
\text { IgG }\end{array}$ & Goat polyclonal & IHC (1:750) & Invitrogen & A-21434 \\
\hline
\end{tabular}

\subsubsection{Plasmids}

Table 2-6: List of plasmids used in this study.

\begin{tabular}{|l|l|l|l|}
\hline Name & Insert & Backbone & Cloning \\
\hline FUGW & EGFP & Addgene \#14883 & $\begin{array}{l}\text { Original vector from } \\
\text { Addgene }\end{array}$ \\
\hline FUGW-Hebp1 & Hebp1-EGFP & Addgene \#14883 & $\begin{array}{l}\text { Cloning by restriction } \\
\text { digest with EcoRI-HF } \\
\text { and AgeI-HF }\end{array}$ \\
\hline psPax2 & None & Addgene \#12260 & $\begin{array}{l}\text { Original vector from } \\
\text { Addgene }\end{array}$ \\
\hline pCMV-VSV-G & None & Addgene \#8454 & $\begin{array}{l}\text { Original vector from } \\
\text { Addgene }\end{array}$ \\
\hline pLenti-CRISPR-V2-Luc & sgRNA, Cas9 & Addgene \#52961 & $\begin{array}{l}\text { Cloning by restriction } \\
\text { digest with BsmBI }\end{array}$ \\
\hline pLenti-CRISPR-V2-Hebp1-H1 & sgRNA, Cas9 & Addgene \#52961 & $\begin{array}{l}\text { Cloning by restriction } \\
\text { digest with BsmBI }\end{array}$ \\
\hline pLenti-CRISPR-V2-Hebp1-H3 & sgRNA, Cas9 & Addgene \# 52961 & $\begin{array}{l}\text { Cloning by restriction } \\
\text { digest with BsmBI }\end{array}$ \\
\hline
\end{tabular}




\subsection{7 sgRNAs used for CRISPR/Cas9 mediated gene knockout}

Table 2-7: List of sgRNAs for CRISPR/Cas9 experiments.

\begin{tabular}{|l|l|l|l|l|}
\hline Name & $\begin{array}{l}\text { Targeted } \\
\text { gene }\end{array}$ & Species & $\begin{array}{l}\text { Targeted } \\
\text { region }\end{array}$ & Sequence \\
\hline Hebp1-H1 & Hebp1 & Homo sapiens & Exon 2 & CAAGGTCGCAAAGTATGCGG \\
\hline Hebp1-H3 & Hebp1 & Homo sapiens & Exon1/Intron1 & CTAAGCAAAGGGGACAAGGT \\
\hline
\end{tabular}

\subsubsection{Buffers and solutions}

All solutions were prepared in ultrapure Type 1 water (Milli-Q).

Table 2-8: List of buffers and solutions with their composition used in this study.

\begin{tabular}{|c|c|}
\hline Buffer/Solution & Composition \\
\hline Homogenization buffer & $320 \mathrm{mM}$ sucrose, 5 mM Hepes, $\mathrm{pH} 7.4$ \\
\hline TFE buffer & $\begin{array}{l}10 \% \text { tetrafluoroethylene, } 100 \mathrm{mM} \text { ammonium bicarbonate, } \\
\text { pH } 8.0\end{array}$ \\
\hline NP-40 Lysis buffer & $\begin{array}{l}50 \mathrm{mM} \text { Tris, } 150 \mathrm{mM} \mathrm{NaCl}, 1 \% \text { Nonidet } \mathrm{P} 40 \text {, pH 7.4, contains } \\
\text { Pierce protease and phosphatase inhibitors }\end{array}$ \\
\hline TBST & 15 mM Tris- $\mathrm{HCl}, \mathrm{pH}$ 7.4, $150 \mathrm{mM} \mathrm{NaCl}, 0.5$ \% (v/v) Tween 20 \\
\hline Transfer buffer & 200 mM Glycine, 25 mM Tris, $20 \%$ Methanol \\
\hline SDS-PAGE Running buffer & 25 mM Tris-HCl, 192 mM Glycine, 0.1 \% SDS \\
\hline PBS & $\begin{array}{l}2.7 \mathrm{mM} \mathrm{KCl}, 1.5 \mathrm{mM} \mathrm{KH}_{2} \mathrm{PO}_{4}, 137 \mathrm{mM} \mathrm{NaCl}, 8 \mathrm{mM} \mathrm{Na}_{2} \mathrm{HPO}_{4} \text {, } \\
\text { pH } 7.35\end{array}$ \\
\hline $\begin{array}{l}\text { High-concentrated } \\
\text { phosphate buffer }\end{array}$ & $\begin{array}{l}411 \mathrm{mM} \mathrm{NaCl}, 8.1 \mathrm{mM} \mathrm{KCl}, 30 \mathrm{mM} \mathrm{Na}_{2} \mathrm{HPO}_{4}, 5.2 \mathrm{mM} \mathrm{KH}_{2} \mathrm{PO}_{4} \text {, } \\
\text { pH } 7.2\end{array}$ \\
\hline Tyrode's solution & $\begin{array}{l}10 \mathrm{mM} \text { Hepes, } \mathrm{pH} \text { 7.3, } 130 \mathrm{mM} \mathrm{NaCl}, 4 \mathrm{mM} \mathrm{KCl}, 5 \mathrm{mM} \mathrm{CaCl}_{2}, 1 \\
\mathrm{mM} \mathrm{MgCl}_{2}, 48 \mathrm{mM} \text { glucose }\end{array}$ \\
\hline $\begin{array}{l}\text { Coomassie Brilliant Blue } \\
\text { staining solution }\end{array}$ & $\begin{array}{l}0.08 \% \text { Coomassie Brilliant Blue G250, } 1.6 \% \text { ortho-Phosphoric } \\
\text { acid, } 8 \% \text { ammonium sulfate, } 20 \% \text { methanol }\end{array}$ \\
\hline
\end{tabular}




\subsubsection{Cell culture media and solutions}

All cell culture media were prepared and maintained in sterile conditions.

Table 2-9: List of cell culture media and solutions with their composition used in this study.

\begin{tabular}{|c|c|}
\hline Medium/Solution & Composition \\
\hline D10 medium & DMEM, $10 \%$ FBS, $20 \mathrm{mM}$ Glutamine \\
\hline Neuronal plating medium & DMEM/F12, 1x B-27, 2 mM L-Alanyl-L-Glutamine \\
\hline Neuronal serum medium & $\begin{array}{l}\text { Eagle's MEM, 5\% FBS, } 2 \text { mM L-Alanyl-L-Glutamine, 1x MEM- } \\
\text { Vitamine, Mito+Serum extender, 3,8 g/L D-glucose }\end{array}$ \\
\hline GBSS & $\begin{array}{l}1.5 \mathrm{mM} \mathrm{CaCl}_{2} \cdot 2 \mathrm{H}_{2} \mathrm{O}, 4.9 \mathrm{mM} \mathrm{KCl}, 0.2 \mathrm{mM} \mathrm{NaH} \mathrm{PO}_{4} \cdot \mathrm{H}_{2} \mathrm{O}, 11 \mathrm{mM} \\
\mathrm{MgCl}_{2} \cdot 6 \mathrm{H}_{2} \mathrm{O}, 0.3 \mathrm{mM} \mathrm{MgSO} \cdot 7 \mathrm{H}_{2} \mathrm{O}, 130 \mathrm{mM} \mathrm{NaCl}, 2.7 \mathrm{mM} \\
\mathrm{NaHCO}_{3}, 0.8 \mathrm{mM} \mathrm{Na} \mathrm{HPO}_{4}, 5 \mathrm{mM} \text { D-glucose, } 22 \mathrm{mM} \text { HEPES, } \\
\text { pH=7.4 in Milli-Q water }\end{array}$ \\
\hline Papain enzymatic solution & $\begin{array}{l}\text { GBSS, } 11.39 \mathrm{mM} \text { L-cystein, } 0.5 \mathrm{mM} \text { Na EDTA, } \mathrm{pH}=8,1 \mathrm{mM} \\
\mathrm{CaCl} \text {, } 3 \mathrm{mM} \mathrm{NaOH}, 0.1 \mathrm{mg} / \mathrm{mL} \text { Dnaze I, } 1 \% \text { Papain suspension }\end{array}$ \\
\hline Inactivation solution & Neuronal serum medium, $0.25 \%$ BSA, $0.1 \mathrm{mg} / \mathrm{mL}$ Dnaze I \\
\hline Poly-D-lysine solution & $\begin{array}{l}0.01 \mathrm{~g} / \mathrm{L} \text { poly-D-lysine, } 25 \mathrm{mM} \mathrm{H}_{3} \mathrm{BO}_{3}, 6,3 \mathrm{mM} \mathrm{Na}_{2} \mathrm{~B}_{4} \mathrm{O}_{7} \cdot 10 \mathrm{H}_{2} \mathrm{O} \\
\text { in Milli-Q water }\end{array}$ \\
\hline
\end{tabular}

\subsection{Methods}

\subsubsection{Mice}

All animal procedures used in this study fully complied with the guidelines as stipulated in the section 4 of the Animal Welfare Law of the Federal Republic of Germany (section 4 of TierSchG, Tierschutzgesetz der Bundesrepublik Deutschland). 3×Tg-AD mice (B6.129.Thy tr.tg-/-), generated on a mixed 129/sv-C57bl6 genetic background, and control B6;129 (129/sv C57bl6 WT) mice were kindly provided by Prof. Wolfgang Härtig (Paul Flechsig Institute for Brain Research, University of Leipzig, Germany). For preparation of primary neurons, Wistar rats originated from the local animal facility were used. All animals were maintained under 12L/12D cycle with food and water ad libitum. 


\subsubsection{Preparation of soluble brain protein fraction}

Whole brains of male mice (both control and 3×Tg-AD) were collected at 2, 6, 12 and 18 months of age in four biological replicates. Half the brain was used for the IHC analysis (see section 2.2.10). Subcellular fractionation was performed on the other half as described before ${ }^{278}$. Briefly, brain tissue was homogenized by a glass-Teflon homogenizer (RW20-DZM, IKA) in $3 \mathrm{ml}$ of the ice-cold homogenization buffer (containing Pierce protease and phosphatase inhibitors) at $900 \mathrm{rpm}$ for 9 strokes. Thereafter, the homogenate was centrifuged for $2 \mathrm{~min}$ at $3000 \times \mathrm{g}$ at $4^{\circ} \mathrm{C}$ in S100AT4 rotor (SORVALL) to remove cell debris. Next, the supernatant was transferred to a new tube and additionally centrifuged for $12 \mathrm{~min}$ at $14,500 \times g$ in S100AT4 rotor at $4^{\circ} \mathrm{C}$ to obtain the soluble fraction of brain proteins (supernatant). For the preparation of crude mitochondria fraction, the upper synaptosomal pellet was first carefully resuspended in homogenization buffer and removed. Remaining mitochondrial pellet was collected in homogenization buffer (Figure 2-1).

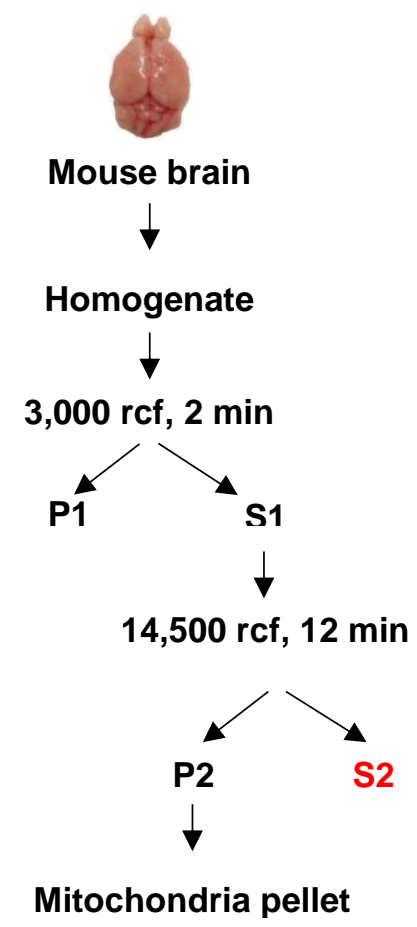

Figure 2-1. Preparation of soluble protein fraction from mouse brain.

Schematic workflow of subcellular brain fractionation. Abbreviations: S1 - supernatant 1, P1 - pellet 1, S2 supernatant 2 (fraction of soluble proteins), P2 - pellet 2 (synaptosomes). 


\subsubsection{Measurement of protein concentration}

To determine the protein concentration, Pierce 660nm Protein Assay Reagent was used according to manufacturer instructions. Protein standard solutions were prepared using albumin in a desired buffer. Protein concentration was determined by measuring the absorbance at $650 \mathrm{~nm}$ in a 96-well plate using the Tecan Genios Pro plate reader according to the manufacturer's manual.

\subsubsection{Preparation of samples for mass spectrometry}

For the proteomic and phosphoproteomic analysis, 40 and $300 \mu \mathrm{g}$ of protein from the soluble fraction were used, respectively. Proteins were precipitated with four volumes of ice-cold acetone overnight at $-20^{\circ} \mathrm{C}$. For global proteomics, the protein pellet was resuspended in $1 \%$ RapiGest and incubated in thermoshaker at $60^{\circ} \mathrm{C}$ for $15 \mathrm{~min}$ at $1050 \mathrm{rpm}$. Disulfide bonds were reduced by $10 \mathrm{mM}$ DTT $\left(60^{\circ} \mathrm{C}\right.$ for 45 minutes at $\left.1050 \mathrm{rpm}\right)$ and alkylated by $25 \mathrm{mM}$ chloroacetamide $\left(37^{\circ} \mathrm{C}\right.$ for 30 minutes at $\left.750 \mathrm{rpm}\right)$. Proteins were then digested by trypsin (1:20 trypsin to protein ratio) in $50 \mathrm{mM}$ ammonium bicarbonate, $\mathrm{pH} 8$, for 16 hours. Digestion was stopped by addition of $1 \%$ formic acid $\left(37^{\circ} \mathrm{C}\right.$ for 1 hour with shaking at 750 rpm) and the peptide solution was cleared by centrifugation $\left(21,800 \times g\right.$ at $4^{\circ} \mathrm{C}$ for 30 minutes). Obtained peptides were desalted using the C18 extraction disk (Sigma, 66883-U), dried in a vacuum concentrator (Savant SPD131DDA, Thermo Fisher Scientific) and stored at $-20^{\circ} \mathrm{C}$ until analyzed by MS.

The phosphoproteomics samples were prepared as described before ${ }^{279}$. Briefly, the acetone precipitated protein pellet was resuspended in TFE buffer using a Bioruptor sonicator (Diagenode). Proteins were then digested by Lys-C $\left(1: 100\right.$ ratio, incubation at $37^{\circ} \mathrm{C}$ for 30 $\min )$ and trypsin (1:50 ratio, incubation at $37^{\circ} \mathrm{C}$ for $15 \mathrm{~h}$ ). Phosphopeptides were enriched using $\mathrm{TiO}_{2}$ beads. The enriched phosphopeptides were desalted using the $\mathrm{C} 18$ extraction disk, dried in the vacuum concentrator and stored at $-20^{\circ} \mathrm{C}$.

\subsubsection{Measurements of mass spectrometry samples}

For the global proteomics analysis, Fusion mass spectrometer (Thermo Fisher Scientific) coupled to Ultimate 3000 HPLC system (Agilent Technologies) was used. Peptides were resuspended in solvent $A$ and loaded onto a trap column packed in-house (100 $\mu \mathrm{m}$ ID $\times 30 \mathrm{~mm}$ self-packed with Reprosil-Pur 120 C18-AQ $1.9 \mu \mathrm{m}$, Dr. Maisch GmbH, 
Ammerbuch-Entringen, Germany) and separated at a flow rate of $300 \mathrm{nl} / \mathrm{min}$ on the analytical column $(75 \mu \mathrm{m}$ ID $\times 300$ mm self-packed with Reprosil-Pur 120 C18-AQ, $1.9 \mu \mathrm{m}$, Dr. Maisch HPLC GmbH, Ammerbuch-Entringen, Germany). Peptides were eluted from the column with 5-76\% gradient of solvent B with an overall run-time of 90 min. Separated peptides were ionized by electrospray ionization (ESI) source in a positive ion mode. Fullscan MS spectra were acquired in the range of $350-1550 \mathrm{~m} / \mathrm{z}$ at a resolution of 60,000 units. The top speed method was selected for fragmentation in the collision cell with Higher-energy Collisional Dissociation with the normalized collision energy of $30 \%$ and isolation window of $1.2 \mathrm{~m} / \mathrm{z}$.

For the phosphoproteomics analysis, enriched phosphopeptides were dissolved in loading buffer (2\% ACN, $0.1 \%$ FA) and analyzed on a Q-Exactive HF hybrid Quadrupole-Orbitrap mass spectrometer (Thermo Scientific) coupled to an Ultimate 3000 RSLC UHPLC (Dionex, USA). The peptides were pre-concentrated on a Reversed Phase-C18 precolumn (100 $\mu \mathrm{m}$ ID $\times 28$ mm self-packed with Reprosil-Pur 120 C18-AQ $1.9 \mu \mathrm{m}$, Dr. Maisch HPLC GmbH) and then separated by reversed phase-C18 nanoflow chromatography (75 $\mu \mathrm{m}$ ID $\times 300 \mathrm{~mm}$ selfpacked with Reprosil-Pur 120 C18-AQ, $1.9 \mu \mathrm{m}$, Dr. Maisch GmbH). Peptides were injected with solvent A $(0.1 \% \mathrm{FA})$ at a flow rate $300 \mathrm{~nL} / \mathrm{min}$ and eluted by $2-60 \%$ gradient of solvent B ( $80 \%$ ACN, $0.08 \%$ FA) with an overall run-time of $90 \mathrm{~min}$. Separated peptides were ionized by ESI source in a positive ion mode. The mass spectrometer was operated in a datadependent acquisition mode. Full-scan MS spectra were acquired in the range of 350-1600 $\mathrm{m} / \mathrm{z}$ at the resolution of 120,000 . The top 15 most intense peaks per cycle from the survey scan were selected for fragmentation in the collision cell with Higher-energy Collisional Dissociation with the normalized collision energy of $28 \%$ and isolation window of $2 \mathrm{~m} / \mathrm{z}$.

\subsubsection{Data processing and bioinformatics analysis}

Acquired MS spectra were processed using the MaxQuant software package version 1.5.0.25280. Spectra were searched using the Andromeda search engine ${ }^{281}$ against the proteome database of Mus musculus (Uniprot complete proteome updated at 2014-05-13, with 24,504 entries). The MaxQuant search was configured as follows: the mass tolerance was set to 20 and $4.5 \mathrm{ppm}$ for the first and the main peptide search, respectively; the multiplicity was set to one; Trypsin/P was fixed as protease and maximum of 2 missed 
cleavages were allowed; carbamidomethylation of cysteine was set as fixed modification and methionine oxidation as well as N-terminal acetylation were specified as variable modifications; a false discovery rate of $1 \%$ was applied; the re-quantification and match between runs options (Match time window $0.7 \mathrm{~min}$, Alignment time window $20 \mathrm{~min}$ ) were enabled. For the phosphoproteomics, phosphorylation on serine/threonine/tyrosine was selected as an additional variable modification.

The "Protein groups" and "Phospho(STY)" output files from MaxQuant was processed by "Perseus", version 1.5.5.3280, for the downstream data analysis. In each time point proteins and phosphosites that were identified in at least two out of four biological replicates in each group (control and disease) were selected for further analysis. Reverse hits were removed. For the phosphoproteomics analysis, reverse hits and phosphosites with localization probability less than 0.75 were removed.

For Principal Component Analysis (PCA) (Figure 3-4), the LFQ intensities were $\log _{2}$ transformed and averaged by group. PCA was performed in "Perseus" with the number of clusters set to 5 and Benjamini-Hochberg FDR cut-off of 0.05 .

For the downstream proteomics analysis, The LFQ intensities of proteins reported by MaxQuant were $\log _{10}$ transformed. The $\log _{2}$ ratio of transformed LFQ intensities AD/Control was calculated for each protein. The LFQ intensities of phosphosites were also $\log _{10}$ transformed and the ratio of $\mathrm{AD} /$ Control intensities for each phosphosite was calculated. The $\mathrm{AD} /$ Control ratio of phosphosites was normalized by $\mathrm{AD} /$ Control ratio of the corresponding protein quantified in the proteome analysis.

Time course changes in biological pathways and their top upstream regulators were identified by Ingenuity Pathway Analysis (IPA, QIAGEN Inc., https://www.qiagenbioinformatics.com/products/ingenuity-pathway-analysis) (Figure 36, Figure 3-7). The $\log _{2} \mathrm{AD} /$ Control intensity ratios of all quantified proteins were used for the analysis with IPA. Positive z-score indicates an overall upregulation (activation) of the process, while a negative score stands for its inhibition. The z-score is computed based on the measured protein expression values ( $\log _{2}$ ratio $\mathrm{AD} /$ control) and the information on the relationship between the proteins and biological processes they are involved in stored in Ingenuity Knowledge Database. 
The IPA was also applied to identify canonical pathways that were activated at time points 2 and 18 months based on phosphoproteomic dataset (Figure 3-10 A). The distribution of significantly up- or downregulated phosphosites by biological processes was determined by Gene ontology (GO) annotation (Figure 3-8 B).

To predict the kinase activity at different time points of AD progression, NetworKIN prediction tool (version 3.0) was used ${ }^{282}$. NetWorKIN predicts substrates of 222 kinases based on the linear motifs and a network context of the kinases. Sequences of mouse phosphosites that were identified as up- or downregulated were matched with homologous human sequences. For the prediction, the following parameters were set: minimum NetWorKIN score of 1.5, maximum difference of 4 and maximum 1 prediction was selected (Figure 3-10 B).

\subsubsection{Analysis of human mRNA expression datasets}

Information on Hebp1 mRNA expression levels in AD patients was extracted from the transcriptome dataset from the Harvard Brain Tissue Resource Center (HBTRC) that is publicly available on the GeneNetwork website (www.genenetwork.org). Used datasets were human primary visual cortex (GN Accession: GN327) and human prefrontal cortex (GN Accession: GN328). These datasets were generated on a custom-made Agilent 44K microarray of 39,280 DNA probes uniquely targeting 37,585 known and predicted genes. The study includes 803 participants of which 388 Alzheimer's disease cases, 220 Huntington's disease cases and 195 controls matched for gender, age and postmortem interval.

\subsubsection{Postmortem human brain samples}

The brain samples were provided by the Prion Disease Surveillance Units of Germany including spAD, rpAD and non-demented control cohorts as described previously ${ }^{283}$. All experimental protocols were approved and the study conformed to the Code of Ethics of the World Medical Association. All study participants or their legal next of kin gave informed consent and the study was approved by the local ethics committee in Göttingen (No. $24 / 8 / 12)$. All samples were anonymized with regard to their personal data. Information on ages, genders, disease duration, disease stage (Braak classification ${ }^{284}$ ) and postmortem interval are summarized in Table 2-10. Brain tissue samples were processed as demonstrated previously 283,285 .

Materials and Methods $\mid 52$ 
Table 2-10. Patient details

\begin{tabular}{|l|l|l|l|l|l|}
\hline $\begin{array}{c}\text { Patients } \\
\text { ID }\end{array}$ & Gender & Age & $\begin{array}{c}\text { Disease duration } \\
\text { (years) }\end{array}$ & $\begin{array}{c}\text { Braak Stages } \\
\text { (AD) }\end{array}$ & $\begin{array}{c}\text { Postmortem } \\
\text { Delays }\end{array}$ \\
\hline Cont. 1 & Male & 86 & - & II/A & $06: 45$ \\
\hline Cont. 2 & Male & 61 & - & I/0 & $03: 03$ \\
\hline Cont. 3 & Male & 74 & - & II/A & $11: 00$ \\
\hline Cont. 4 & Male & 86 & - & II/A & $06: 45$ \\
\hline Cont. 5 & Female & 73 & - & I/0 & $04: 03$ \\
\hline Cont. 6 & Male & 69 & - & II/A & $05: 03$ \\
\hline Cont. 7 & Male & 68 & - & I/0 & $05: 03$ \\
\hline Cont. 8 & Female & 64 & - & I/0 & $09: 00$ \\
\hline Cont. 9 & Male & 67 & - & I/0 & $05: 03$ \\
\hline spAD1 & Female & 72 & $>4$ & V/C & $09: 30$ \\
\hline spAD2 & Female & 75 & $>4$ & V/C & $04: 15$ \\
\hline spAD3 & Male & 78 & $>4$ & V/C & $09: 30$ \\
\hline spAD4 & Male & 83 & $<4$ & V/C & $08: 20$ \\
\hline spAD5 & Female & 56 & $>4$ & V/C & $07: 00$ \\
\hline spAD6 & Male & 83 & $>4$ & III/0 & $07: 25$ \\
\hline spAD7 & Female & 90 & $>4$ & IV/A & $09: 55$ \\
\hline spAD8 & Female & 93 & $>4$ & V/C & $03: 00$ \\
\hline rpAD1 & Male & 78 & $<4$ & V/C & $03: 30$ \\
\hline rpAD2 & Female & 79 & $<4$ & V & $05: 30$ \\
\hline rpAD3 & Female & 81 & $<4$ & III/B & $06: 00$ \\
\hline rpAD4 & Male & 83 & $<4$ & VI/C & $05: 30$ \\
\hline rpAD5 & Male & 83 & $<4$ & V/C & $08: 20$ \\
\hline rpAD6 & Male & 70 & $<4$ & VI/C & $11: 30$ \\
\hline rpAD7 & Male & 76 & $<4$ & VI/C & $06: 30$ \\
\hline rpAD8 & Female & 77 & $<4$ & & $12: 00$ \\
\hline
\end{tabular}

\subsubsection{Immunoblotting}

Immunoblotting was performed according to standard procedures ${ }^{286}$. Protein samples were mixed with 4× NuPAGE LDS Sample Buffer (Thermo Fisher Scientific, NP0008) and boiled at $95^{\circ} \mathrm{C}$ for 5 minutes. $15 \mu \mathrm{g}$ of protein sample were typically loaded on per lane of a gel. Mouse brain samples and Co-IP samples were separated on 12\% SDS-PAGE gels. Proteins were transferred to nitrocellulose membrane using the Mini Trans-Blot Cell system (Bio-Rad) for 1 hour at constant voltage of $100 \mathrm{~V}$. Human samples were run on $4-20 \%$ Criterion TGX gels (Bio-Rad, 5671095) and transferred using Trans-Blot Turbo Transfer System (Bio-Rad). 
Membranes were blocked in 5\% non-fat dry milk in TBST for 1 hour at room temperature, incubated with primary antibodies in blocking solution overnight, washed with TBST (5 times, 5 minutes each), incubated with secondary antibodies for 1 hour at room temperature and washed again with TBST ( 5 times, 5 minutes each). Protein bands were visualized using fluorescence or enhanced chemiluminescence and images were developed using Odyssey CLx Infrared Imaging System (Licor) or Fujifilm LAS-100 device, respectively.

\subsubsection{Immunohistochemistry}

Mouse tissue samples for immunohistochemistry were prepared as described previously 287. In brief, for the preparation of cryosections, one half of the dissected whole brain was fixed in $4 \%$ PFA for 4 hours at $4^{\circ} \mathrm{C}$ and washed in PBS three times for 20 minutes each. Tissues were immersed in 15\% sucrose in PBS (1 hour), followed by 30\% sucrose in PBS (overnight) and finally in 50\% tissue freezing medium (Tissue Tek, Leica) in 30\% sucrose for 1 hour. Tissue was embedded in the freezing medium, frozen at $-20^{\circ} \mathrm{C}$ and preserved at $-80^{\circ} \mathrm{C}$ until use.

For paraffin sections, whole brains of 12-month-old mice were fixed in 4\% PFA overnight, washed in PBS three times (20 minutes each) and subsequently immersed in $0.98 \% \mathrm{NaCl}$ for 1 hour. The tissues were then dehydrated in a stepwise series of ethanol dilutions (50\%, 70\%, 90\%, 95\%, 100\%), cleared in the ascending toluene/isopropanol dilution series and finally embedded in paraffin.

Immunostainings were performed on $10 \mu \mathrm{m}$ thick cryo-sections. Sections were washed three times in PBS and blocked in 10\% FCS and 0.5\% Triton-X100 in PBS for 60 min at room temperature. Slides were incubated overnight with primary antibodies at $4^{\circ} \mathrm{C}$ in the blocking solution followed by three washes in PBS (10 minutes each) and incubation with secondary antibodies (1: 750) for 60 minutes at room temperature. Finally, sections were rinsed in PBS three times (10 minutes) and mounted with Vectashield containing DAPI (Vector Laboratories). Additional $8 \mu \mathrm{m}$ thick paraffin sections were used for IBA1/Hebp1 co-staining. Prior to the staining, paraffin sections were hydrated through descending ethanol series and boiled for one minute in unmasking solution (1:100 in water, Vector Laboratories, H-3300). 


\subsubsection{Staining of $A \beta$ plaques}

$\mathrm{A} \beta$ plaques were stained with thioflavin S (Santa Cruz, CAS 1326-12-1) as described previously ${ }^{288}$. Briefly, $10 \mu \mathrm{m}$ thick cryo-sections were equilibrated to room temperature and rinsed in PBS for 3 minutes. The slides were stained with filtered $0.05 \%$ thioflavin S in $50 \%$ ethanol for 8 minutes in the dark and differentiated with $80 \%$ ethanol (2 times for 10 seconds). Subsequently, the sections were washed with large volumes of distilled water and incubated in high-concentrated phosphate buffer at $4^{\circ} \mathrm{C}$ for 30 minutes. Finally, slides were briefly rinsed in PBS and mounted with Vectashield containing DAPI. Slides were allowed to set in the dark at room temperature and were imaged immediately thereafter using Zen Observer 1 microscope (Zeiss).

\subsubsection{Primary neurons and cell culture}

Primary cortical or hippocampal neurons were prepared from postnatal day 0 Wistar rats. Dissected cortices and hippocampi were digested for $30 \mathrm{~min}$ with papain enzymatic solution. Digestion was stopped by addition of inactivation solution. Digested tissues were triturated using a fire-polished Pasteur pipette until no visible tissue debris could be observed. The cell suspension was passed through a $40 \mu \mathrm{M}$ cell strainer (Corning Costar, 352340) and subsequently centrifuged for $5 \mathrm{~min}$ at $500 \mathrm{rpm}$ followed by resuspension of cell pellets in serum medium. Next, cortical neurons were plated in plating directly on PDL-coated $10 \mathrm{~cm}$ culture plates (Greiner, 664160) for Co-IP analysis (1 cortex per plate). Hippocampal neurons were plated on PDL-coated coverslips in plating medium at a density of 20,000 cells $/ \mathrm{cm}^{2}$ for imaging. Medium was changed completely to fresh plating medium supplemented with $1 \times$ FUDR the next day.

All cells were grown at $37^{\circ} \mathrm{C}$ in a humidified incubator with $5 \% \mathrm{CO}_{2}$ atmosphere. HEK293 and HeLa cells were cultured in D10 medium.

Mitochondria isolation from HEK293 cells was performed exactly as described before ${ }^{289}$.

\subsubsection{Lentiviral transduction}

For overexpression of Hebp1 in primary rat neurons, cDNA encoding full-length human Hebp1 was subcloned to FUGW backbone (FUGW was a gift from David Baltimore, Addgene plasmid \# 14883) using EcoRI and AgeI restriction enzymes. Empty FUGW vector was used as a negative control for overexpression of EGFP. For production of lentiviral particles, 
HEK293 cells were co-transfected with the FUGW-Hebp1or FUGW plasmid, and the helper plasmids psPax2 (a gift from Didier Trono, Addgene plasmid \#12260) and pCMV-VSV-G (a gift from Bob Weinberg, Addgene, plasmid \#8454) in a 2:1:1 ratio using Lipofectamine 2000. Medium was changed 6 hours after transfection to DMEM supplemented with 2\% FBS and 5 mM sodium butyrate. Culture supernatant was harvested 24 hours and lentiviruses concentrated by ultracentrifugation via Amicon Ultra-15 filters (Millipore, UFC910024). Concentrated lentiviruses were diluted to the final volume of $1 \mathrm{~mL}$ in DMEM/F12 medium, aliquoted, snap-frozen in liquid nitrogen and stored at $-80^{\circ} \mathrm{C}$ until use. Only lentivirus preparations resulting in transduction rate of at least $90 \%$ (assessed by EGFP overexpression) were used for experiments.

\subsubsection{Live imaging of mitochondria}

Primary rat hippocampal neurons were infected with lentiviruses overexpressing Hebp1EGFP or EGFP alone one day after seeding and analyzed at DIV14. Prior to imaging, cells were incubated with MitoTracker Red CMXRos (Life Technologies, M5712) in plating medium at final concentration of $10 \mathrm{nM}$ for minimum of 30 minutes. Cells were then imaged in Tyrode's solution using Zeiss Observer 1 laser scanning confocal microscope within a 30-minute period.

\subsubsection{Identification of Hebp1 binding partners}

Proteins interacting with Hebp1 were identified using co-immunoprecipitation coupled with mass spectrometry in four independent biological experiments. Primary rat cortical neurons were infected with lentiviruses expressing Hebp1-EGFP or EGFP one day after seeding. Neurons were lysed at DIV14 with NP-40 lysis buffer. Lysates were clarified by centrifugation at $13,000 \times \mathrm{g}$ for $10 \mathrm{~min}$ at $4^{\circ} \mathrm{C}$. Hebp1-EGFP and EGFP were pulled down using GFP-trap according to manufacturer's instructions. Beads were sequentially washed in lysis buffer containing descending concentrations of NP-40 (1\%, 0.8\%, 0.4\%, 0.2\%). Proteins were eluted by boiling the beads at $95^{\circ} \mathrm{C}$ for $10 \mathrm{~min}$ in $1 \times$ NuPAGE LDS Sample Buffer and separated on 4-12\% Bis-Tris NuPAGE gels (Thermo Fisher Scientific, NP0342). Gels were stained with Coomassie solution overnight and destained in deionized water for two days. Each lane was cut into six equal pieces and in-gel protein digestion was performed as described 
previously ${ }^{290}$. Peptides extracted from each gel piece were measured three times in independent technical repetitions.

The digested peptides were subjected to Q Exactive HF mass spectrometer (Thermo Fisher Scientific) coupled with an Ultimate 3000 RSLC system (Dionex, USA). Peptides were separated on a self-made capillary column (ReproSil-Pur 120 C18-AQ, $1.9 \mu \mathrm{m}$, Dr. Maisch $\mathrm{GmbH}, 300$ x $0.075 \mathrm{~mm}$; C18 pre-column from Thermo Fisher (article nr. 160454)) with a 5$42 \%$ linear gradient of increasing buffer B ( $80 \% \mathrm{ACN}, 0.08 \% \mathrm{FA})$ and decreasing buffer $\mathrm{A}$ ( $0.1 \% \mathrm{FA}$ in water) for an overall run time of $58 \mathrm{~min}$ at a constant flow rate of $300 \mathrm{nl} / \mathrm{min}$. Separated peptides were ionized by ESI source in a positive ion mode. Full-scan MS spectra were acquired in the range of $350-1600 \mathrm{~m} / \mathrm{z}$ at the resolution of 60,000 units. The top 30 most abundant precursors were selected for fragmentation in the collision cell with Higherenergy Collisional Dissociation with the normalized collision energy of 30\% and isolation window of $1.6 \mathrm{~m} / \mathrm{z}$. Max quant search was performed with the same parameters used for brain proteome analysis.

Perseus software was used for downstream data analysis. The intensities of identified proteins were $\log _{2}$ transformed and the missing values for identified proteins in each replicate were imputed with the width of 0.3 and downshift of 1.8 in the total matrix mode. $\log _{2}$ difference between Hebp1-EGFP and EGFP samples was calculated for each identified protein and was averaged between technical and biological replicates. Statistical significance of protein enrichment in each sample was determined by one-sample t-test $(\mathrm{p}<0.05)$.

\subsubsection{Generation of Hebp1 knockout HeLa lines by CRISPR/Cas9 system}

sgRNA (5'-CTAAGCAAAGGGGACAAGGT-3') targeting human Hebp1 was designed using sgRNA Designer (Broad Institute). The pLenti-CRISPR-H3 vector was generated by inserting the sgRNAs into the LentiCRISPRv2 plasmid at the BsmBI site. The LentiCRISPRv2 plasmid is a gift from Feng Zhang (Addgene, plasmid \#52961). To obtain stable Hebp1 knockout HeLa lines, HeLa cells were transiently transfected with pLenti-CRISPR-H3 construct targeting human sequence of Hebp1 using Lipofectamine 2000. Twenty-four hours following transfection, the cells were subjected to puromycin selection $(2 \mu \mathrm{g} / \mathrm{mL})$ for 2 days. Selected cell colonies were picked, expanded and subsequently tested for Hebp1 expression by 
immunoblotting. Knockout and wild type lines (unaltered Hebp1 expression) were selected and propagated for further experiments.

\subsubsection{In vitro cell-based assays}

HeLa cells were seeded on black 96-well plates (Corning) at a density of $3 \times 10^{4}$ cells $/ \mathrm{cm}^{2}$. Cell toxicity was assessed using MultiTox-Glo reagent according to the manufacturer's instructions. Briefly, cytotoxicity was measured by activity of dead-cell protease (luminescent readout) and was normalized to cell viability measured by the activity of livecell protease (fluorescent readout) to account for discrepancies in cell number between the wells. Final cytotoxicity values are presented as a fold change of corresponding vehicle control. Cells were treated with $10 \mu \mathrm{M}$ hemin, $100 \mu \mathrm{M}$ tert-butyl-hydroperoxide or $1 \mu \mathrm{M}$ staurosporine. Hemin was always prepared fresh in accordance with a protocol published previously 291.

Apoptosis was assessed by measurement of caspase 3/7 activity using CellEvent Caspase3/7 Green Detection Reagent. The Caspase-3/7 Green Detection Reagent was added to cells simultaneously with hemin treatment. Apoptotic cells were quantified 24 hours later. Images were acquired from three non-overlapping fields of each well with a 10× objective, Zeiss Axiovert 200M (GFP channel and brightfield). Cells positive for Caspase-3/7 activity were quantified with in-house written macro in Fiji ${ }^{292}$. Total number of cells was quantified manually from brightfield images. Each experiment was performed at least in three independent biological repetitions with three technical replicates for each condition.

Levels of oxidative stress were determined by measurement of reduced/oxidized glutathione ratio (GSH/GSSG) using GSH/GSSG-Glo Assay according to the manufacturer's guidelines. Luminescence was assessed twenty-four hours after treatment with hemin by Tecan Genios Pro plate reader with integration time and time between move and integrations set to 100 $\mathrm{ms}$ and $500 \mathrm{~ms}$, respectively. Reduced (GSH) and oxidized (GSSG) glutathione were measured in separate wells (two technical replicates each). The ratio GSH/GSSG was calculated thereafter. Each experiment was performed in five independent experiments.

Mitochondrial membrane potential was assayed with the corresponding kit from Cell Signaling according to the manufacturer's guidelines. Briefly, potentiometric fluorescent TMRE dye (final concentration $200 \mathrm{nM}$ ) was added to HeLa cells twenty-four hours after Materials and Methods $\mid 58$ 
hemin treatment. Fluorescence was measured 30 minutes after with Tecan Genios Pro plate reader using $550 \mathrm{~nm}$ and $615 \mathrm{~nm}$ excitation and emission filters, respectively.

Statistical analysis of the data obtained in in vitro cell-based assays was performed using Prism software. Applied statistical tests with major parameters are mentioned in the legends of corresponding figures. 


\section{Results}

\subsection{Experimental design}

The first objective of my project was to characterize the brain proteome and phosphoproteome of Alzheimer's disease over the course of its progression, including the presymptomatic stage. In order to do that, an optimal biological system to study the disease and its development needed to be selected. The use of brain samples from patients would be the most appropriate to investigate a human disease. However, the available samples are limited to postmortem brain tissue that captures only the terminal stage of the disease. The use of animal models is thus a viable alternative. The pros and cons of various mouse models of $\mathrm{AD}$ were discussed in detail in the Introduction (section 1.2). Among available mouse models, I selected the triple transgenic AD mice ( $3 \times \mathrm{Tg}-\mathrm{AD})$ as they fulfill three important criteria that align with the aims of my work. First, $3 \times$ Tg-AD mice exhibit both key molecular hallmarks of AD pathology: amyloid beta plaques and hyperphosphorylated tau. Second, the overexpression of human transgenes (APPswe, MAPT ${ }_{\mathrm{P} 301 \mathrm{~L}}$ ) in $3 \times \mathrm{Tg}-\mathrm{AD}$ is under the control of mouse Thy1.2 promoter and is rather mild $(\sim 1.5-2$ fold of endogenous APP and MAPT expression) in comparison to other mouse models. The third and the most important reason for choosing the $3 \times \mathrm{Tg}$-AD model for this study is that these mice develop the disease in a progressive age-dependent manner closely resembling $\mathrm{AD}$ in humans.

To identify the proteins and phosphosites differentially expressed over the course of AD progression, I analyzed brain proteomes of age-matched $3 \times \mathrm{Tg}-\mathrm{AD}$ and control mice at four distinct time points using label-free quantitative mass spectrometry. The four time points were selected according to the well-characterized progressing pathological changes in $3 \times \mathrm{Tg}$ AD mice based on previously published data ${ }^{233,293}$. Additionally, I confirmed the presence of $\mathrm{A} \beta$ plaques and hyperphosphorylated tau in the brains of $3 \times \mathrm{Tg}-\mathrm{AD}$ mice from our colony (Figure 3-1). The selected four time points include the presymptomatic time point (2 months), the age of first behavioral abnormalities ( 6 months), appearance of first $A \beta$ plaques (12 months) and hyperphosphorylated tau (18 months) (Figure 3-2 A). 
A
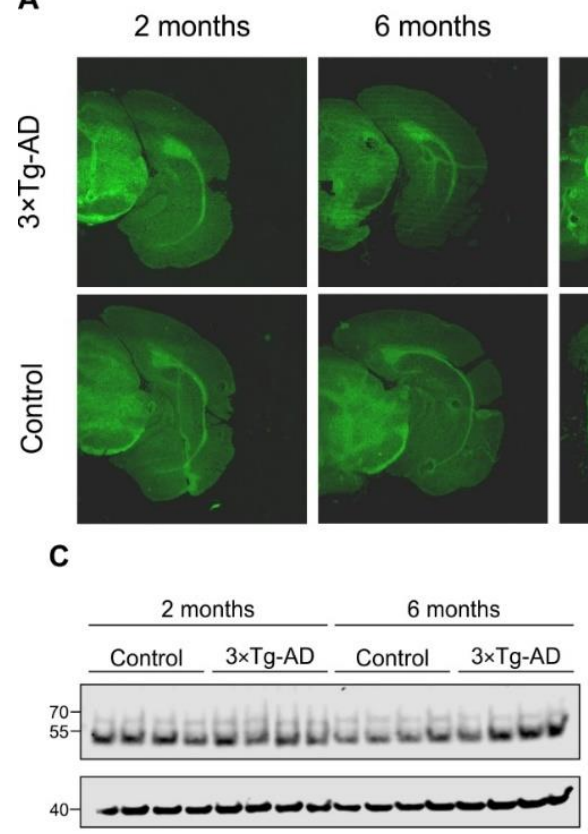

D

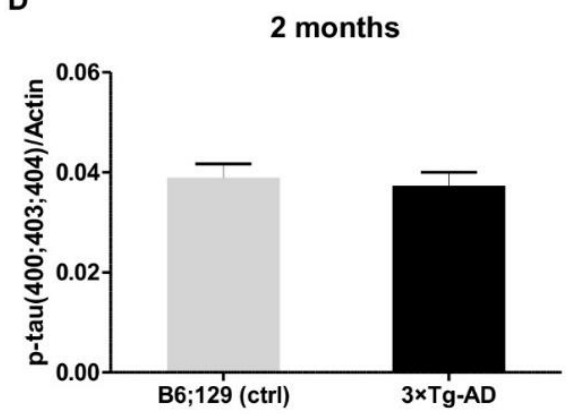

E

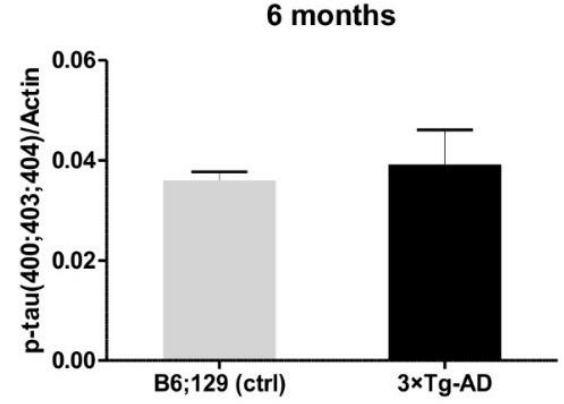

12 months
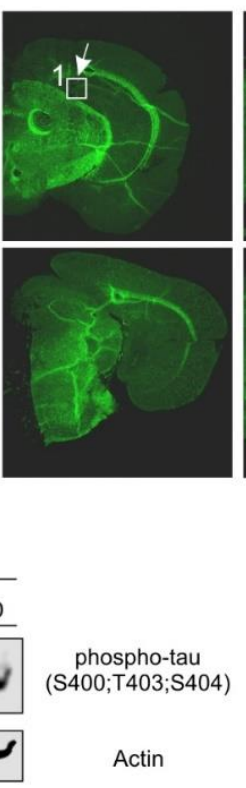

$\mathbf{F}$
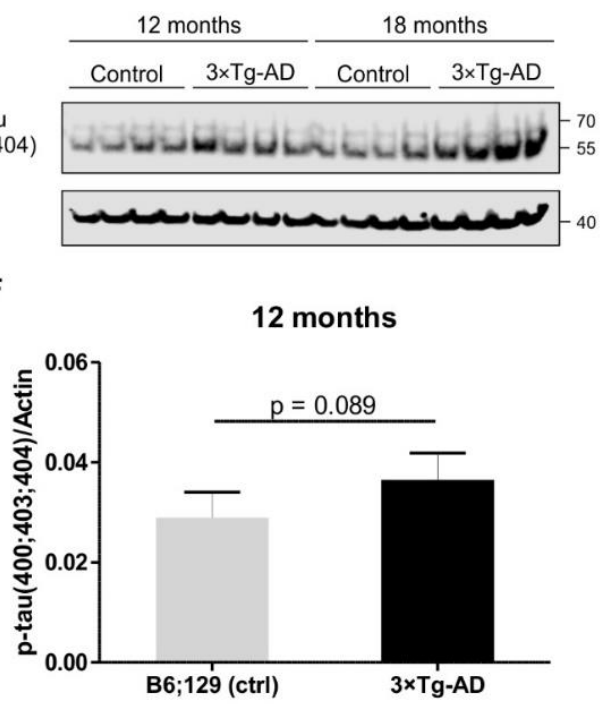

G

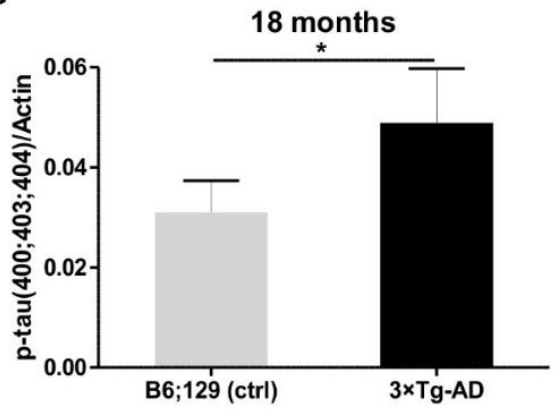

Figure 3-1. Assessment of pathology in 3×Tg-AD mice used in this study.

(A) Accumulation of $A \beta$ plaques assayed by thioflavin $S$ staining at each analyzed time point in $3 \times \mathrm{Tg}-\mathrm{AD}$ and control mice. Representative images of coronal sections including subiculum region were acquired. White arrows indicate exemplary A $\beta$ plaques. (B) High magnification images of $A \beta$ plaques in 12 and 18-month-old $3 \times$ Tg-AD mice.

(C) Assessment of tau phosphorylation at sites S400/T403/S404 by immunoblotting. Soluble fraction of brain proteins was used for the analysis. (D-G) Relative quantification of tau phosphorylation. Slight upregulation of phospho-tau can be observed at the time point 12 months in $3 \times$ Tg-AD mice. Significantly different levels of tau phosphorylation were detected at the late stage of the disorder (18 months).

All bar charts represent mean \pm SD. Statistical significance in the datasets was assessed by Student's t-test: ${ }^{*} p$ value $<0.05$. 


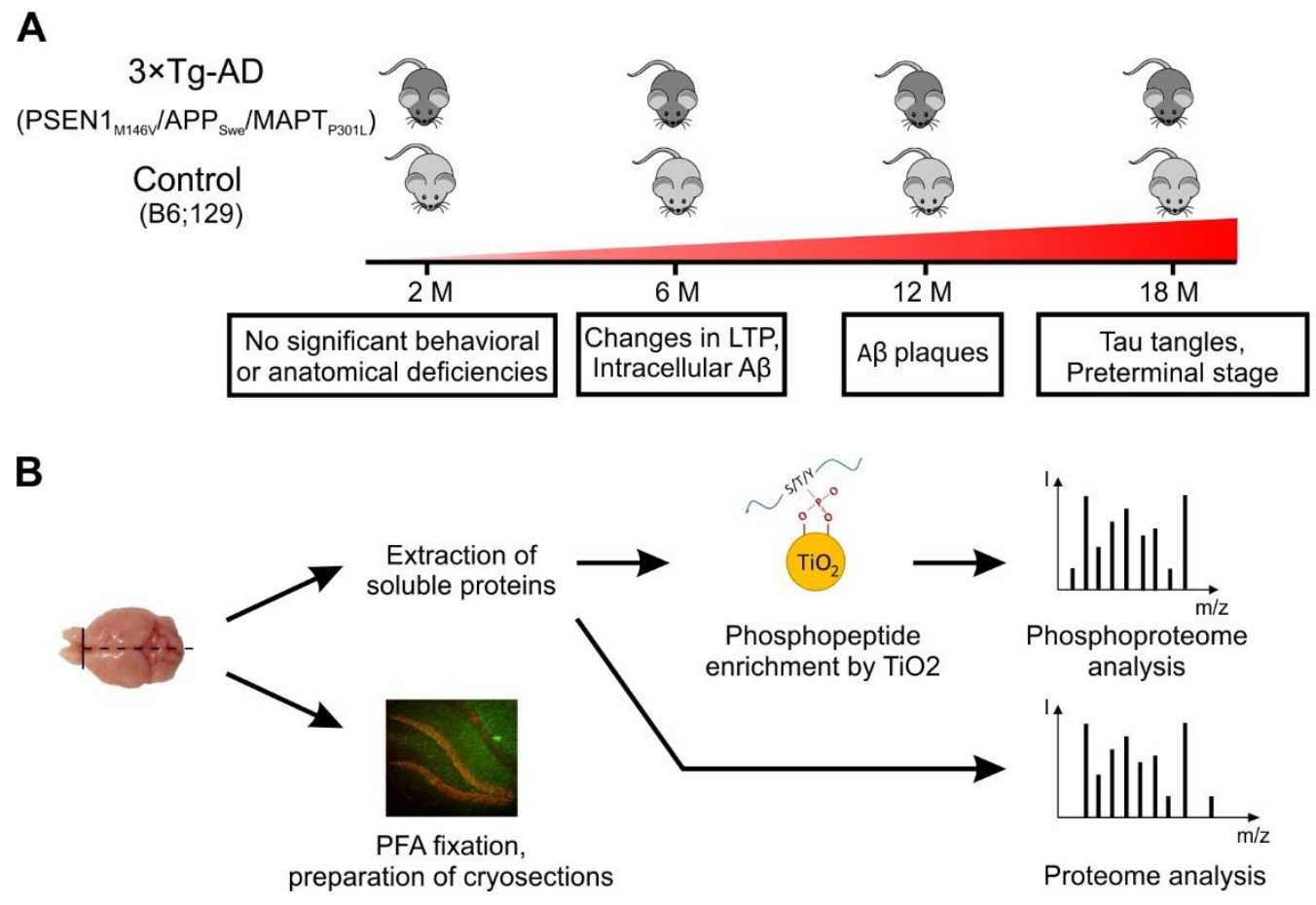

Figure 3-2. Experimental design.

(A) Disease progression in $3 \times \mathrm{Tg}-\mathrm{AD}$ mice and corresponding time points $(2,6,12,18$ months) of sample collection in this study. Four biological replicates per group ( $3 \times \mathrm{Tg}-\mathrm{AD}$ and control) were collected at each time point. (B) Schematic representation of brain sample processing.

Whole mouse brains were collected at the designated time points. One half of the brain was processed to obtain a soluble protein fraction (Figure 2-1). For proteome analysis, $40 \mu \mathrm{g}$ of this fraction were digested by trypsin and subjected to analysis by label-free liquid chromatography-tandem mass spectrometry (LC-MS/MS) (Figure 3-2 B). In parallel, $300 \mu \mathrm{g}$ of the soluble protein fraction were digested and utilized for phosphopeptide enrichment with titanium dioxide beads and further analysis by label-free LC-MS/MS to map the brain phosphoproteome. The remaining half of the brain was used to prepare cryosections for subsequent immunohistochemical assessment of hits identified by mass spectrometry (see Methods for exact description of experimental procedures).

\subsection{Assessment of brain proteome in $3 \times \mathrm{Tg}-\mathrm{AD}$ mice along AD progression}

In total, 3760 proteins were identified in the soluble brain protein fraction (peptide and protein false discovery rate (FDR) of 1\%). Additional stringency was applied by selecting 
A

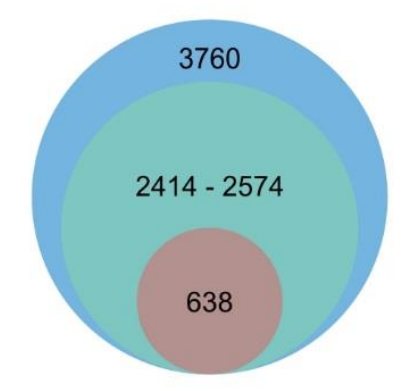

Identified proteins

Quantified proteins

Statistically significant proteins (t-test, $p<0.05)$ at least in one time point
B
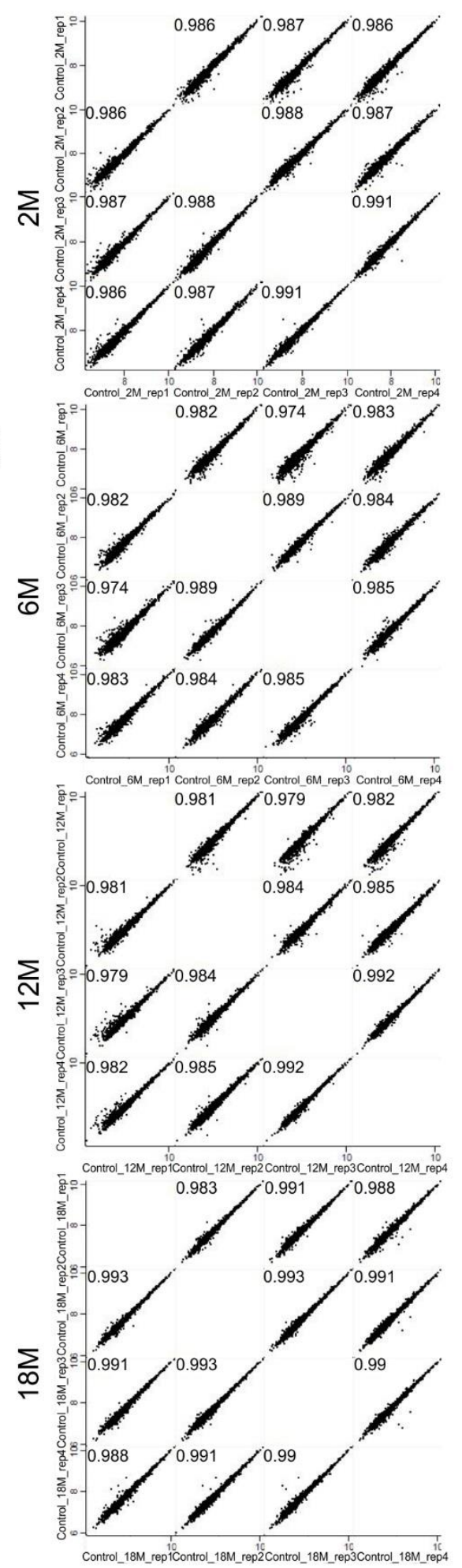

$3 \times \operatorname{Tg}-\mathrm{AD}$
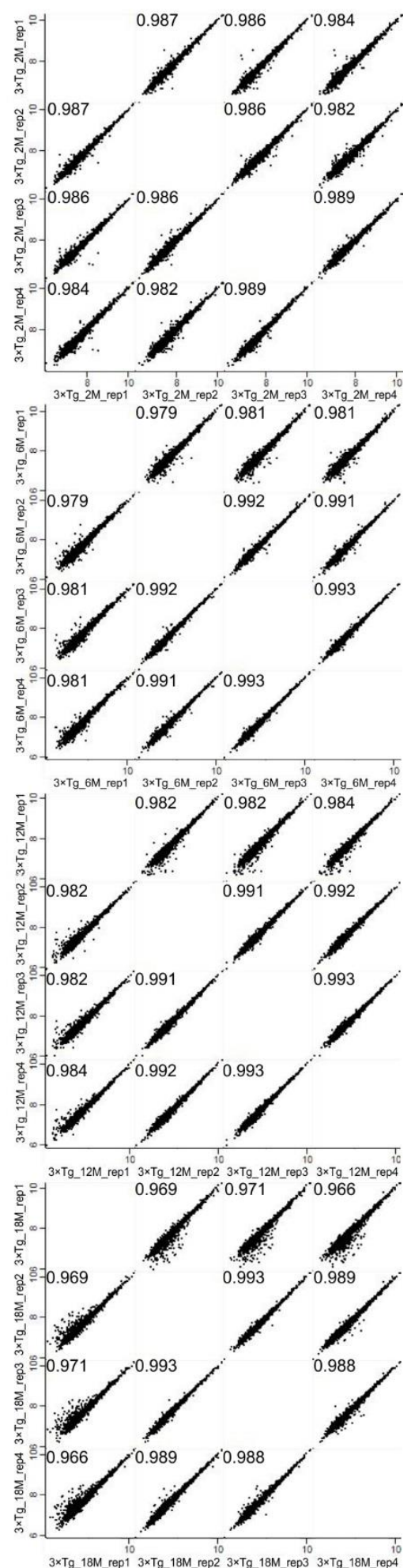

Figure 3-3. Number of identified proteins and technical reproducibility of proteomic data.

(A) Cumulative number of identified, quantified and statistically significant proteins at all time points.

(B) Pearson correlation coefficients between biological quadruplicates for each condition and time point. 
proteins that were present in at least two out of four biological replicates in each group (disease or control). Thus, between 2414 and 2574 proteins were quantified and further analyzed depending on the time point (Figure 3-3 A). Pearson's correlation coefficients for quantified proteins between biological replicates were above 0.96 , attesting to the high reproducibility of the data (Figure 3-3 B).

\subsubsection{Principal component analysis clusters proteins by age and disease status}

Principal component analysis (PCA) of the datasets revealed that the quantified proteins could be clustered according to age (component 1, 40.9\% of total variation) as well as disease state (component 2, 18.3\% of total variation) (Figure 3-4 A). Notably, segregation by age was mainly driven by extracellular matrix proteins (Hapln2, Tnc, Acan, Vcan, Hapn1) and increased expression of microglia markers (S100b, Ctpd) (Figure 3-4 B). Many of these proteins have been previously reported as markers of brain aging294,295. The samples segregated by principle component 2 varied primarily in the expression of AD-related genes. As expected, APP and tau (MAPT) were detected in the AD cluster (Figure 3-4 B). The PCA also demonstrated that differences between the brain proteomes of $3 \times \mathrm{Tg}$ - $\mathrm{AD}$ and control mice increased with disease progression as indicated by the growing distance between the points corresponding to diseased and control mice of the same age (Figure 3-4 A).

\subsubsection{Relative quantification of protein expression}

After a general assessment of proteomic data with PCA, I investigated the changes in the protein expression of individual proteins between diseased and control mice at each stage of $A D$. For this purpose, the $\log _{2}$ ratio of $L F Q$ intensities between $3 \times T g-A D$ and control mice was calculated ( $\log _{2}$ ratio $\mathrm{AD} /$ Control). Statistical significance of expression change was determined by t-test ( $\mathrm{p}$-value cut-off of $<0.05$ for statistically significant difference). Volcano plots were built based on the $\log _{2}$ ratio $\mathrm{AD} /$ Control and the $-\log _{10}$ of $\mathrm{p}$-value for each quantified protein. (Figure 3-5 A-D). Statistical analysis demonstrates that 7 to $8 \%$ of quantified proteins were expressed at significantly different levels between $3 \times \mathrm{Tg}-\mathrm{AD}$ and control mice at time points 2,6 and 12 months. The percentage of significantly regulated proteins almost doubled (13.7\%) by the preterminal stage of the disease (18M). This result confirms that brain proteome of $3 \times \mathrm{Tg}-\mathrm{AD}$ mice is more affected at the late stage of the disease. At the same time these data also indicate that proteome aberrations occur early in 
the disease progression and can be already observed at the presymptomatic stage (2 months).
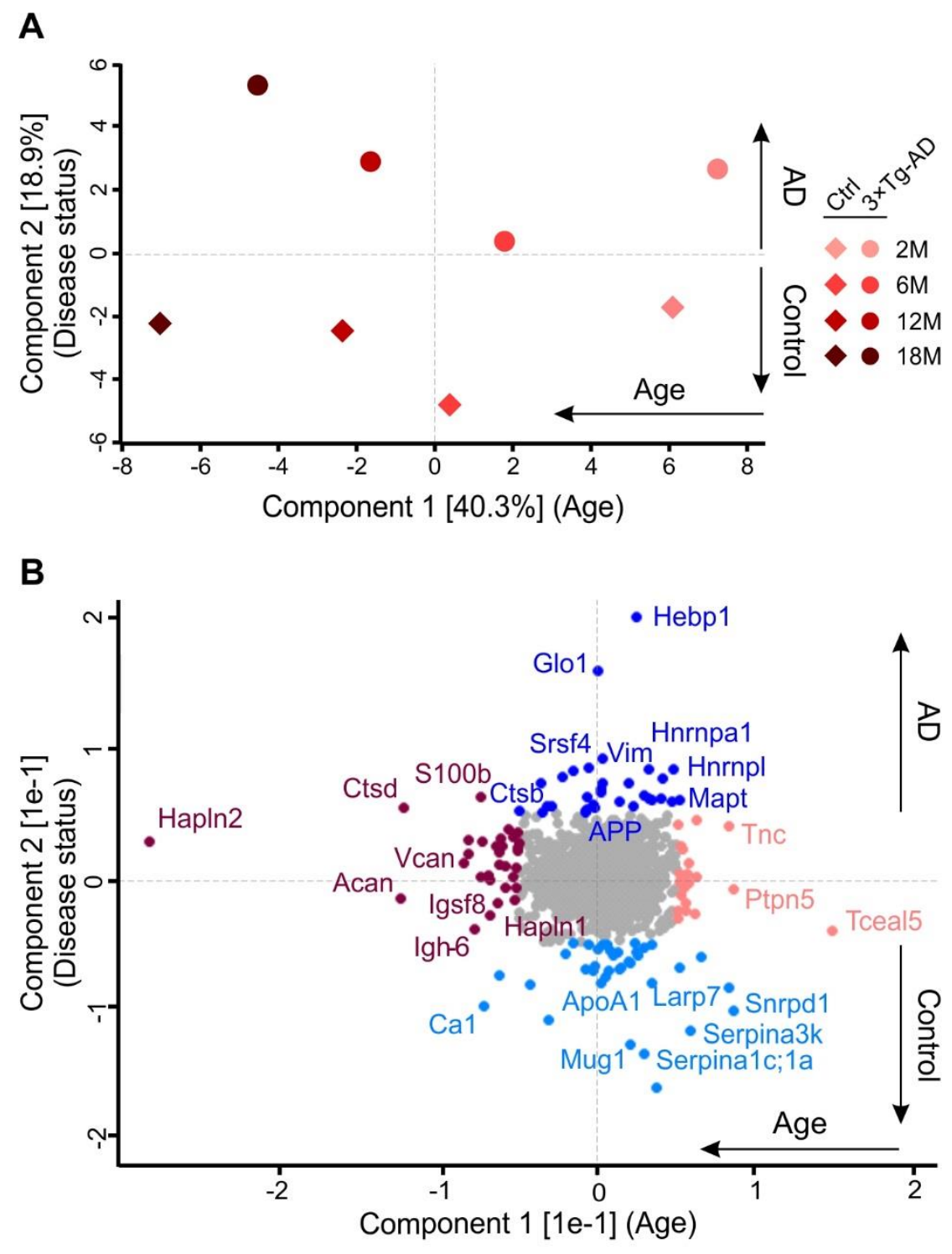

Figure 3-4. Principal component analysis reveals disease- and age-dependent alterations of $3 \times \mathrm{Tg}$-AD brain proteome.

(A) Principal component analysis of soluble brain proteome of $3 \times \mathrm{Tg}-\mathrm{AD}$ and control mice based on their protein expression profile. Principal component 1 segregates mice by age and accounts for $40.3 \%$ of variability in the dataset, while principal component 2 clusters mice according to their disease status (18.9\% of variance). (B) Proteins driving the difference between aged, young, diseased and control mice are depicted in brown, pink, navy and light blue colors, respectively. 

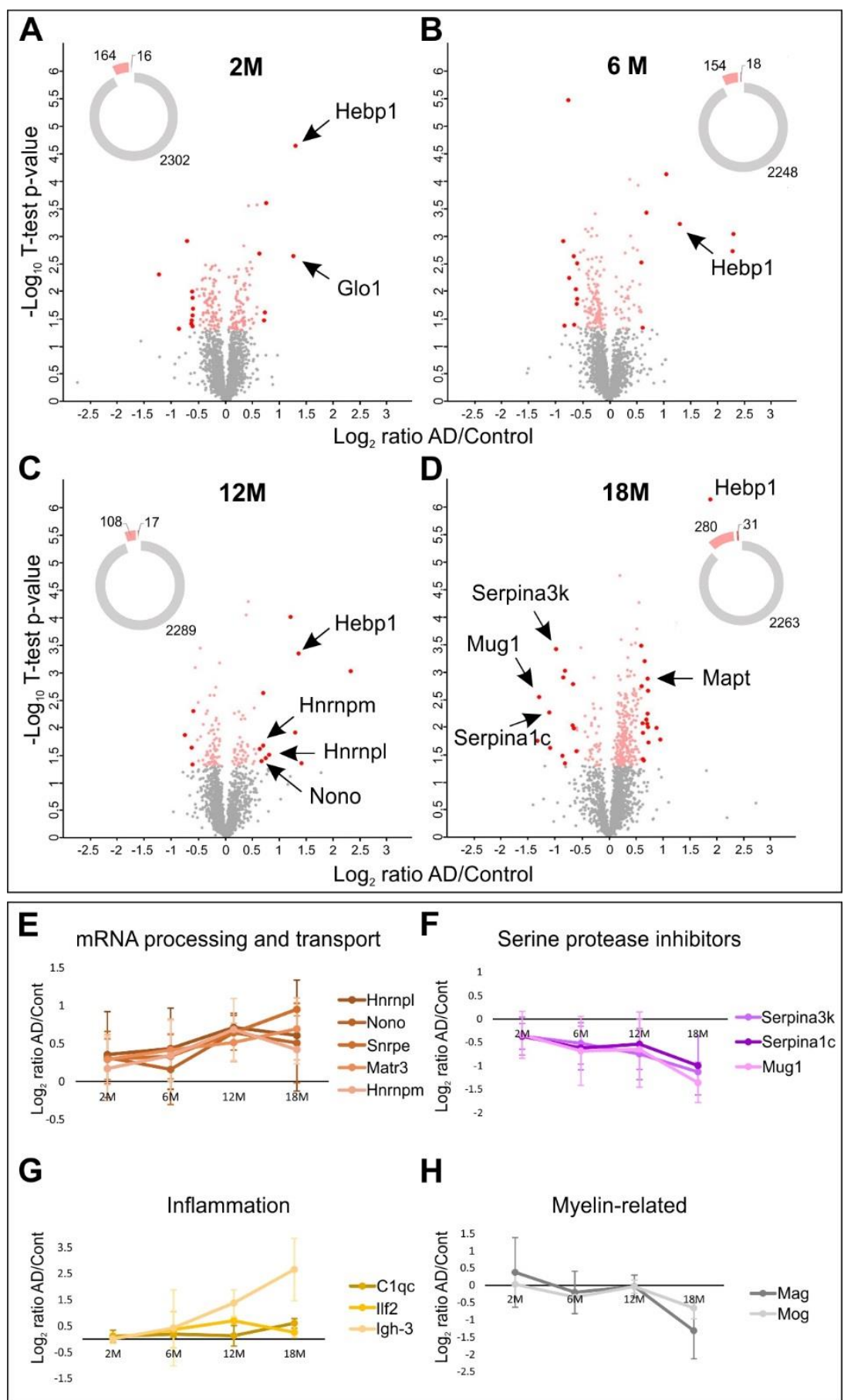

Figure 3-5. Comparative proteome analysis of $3 \times \mathrm{Tg}-\mathrm{AD}$ and control samples at different stages of $\mathrm{AD}$. Distribution of the quantified proteins at $2 \mathrm{M}(\mathrm{A}), 6 \mathrm{M}(\mathrm{B}), 12 \mathrm{M}(\mathrm{C})$ and $18 \mathrm{M}(\mathrm{D})$ based on $\log _{2}$ ratio $\mathrm{AD} /$ Control and $-\log _{10} \mathrm{p}$-value ( $\mathrm{t}$-test) by time point. The pie charts represent the number of: quantified non-regulated proteins (grey), significantly different proteins between AD and control samples, t-test p-value $<0.05$ (pink), and significantly regulated proteins with more than $50 \%$ change (red). Dynamics of the expression over the course of $\mathrm{AD}$ progression for selected highly regulated proteins based on their function: proteins involved in mRNA processing and transport (E) and inflammation (G) that are upregulated over the course of disease progression, and serine protease inhibitors $(\mathrm{F})$ and myelin-related proteins $(\mathrm{H})$ that are downregulated. 
To identify potential markers indicative of disease onset and progression, the dataset was scanned to determine individual proteins with the highest degree of fold change at each AD stage. Such proteins were selected based on two criteria: 1) statistical significance (t-test, p $<0.05$ ), and 2) greater than $50 \%$ change in expression level between disease and control (Figure 3-5 A-D, red dots). These highly regulated proteins could be grouped based on their function. For example, several proteins involved in mRNA processing (Hnrnpm, Hnrnpl, Nono, Matrin3, Snrpe) were strongly upregulated at late time points (12 and 18 months). Remarkably, the expression of these proteins changed in a coordinated fashion gradually increasing throughout the progression of the disease (Figure 3-5 E). A similar coordinated expression pattern was also observed for the group of serine protease inhibitors (Serpina1c, Serpina3k, Mug1) which were significantly downregulated by the late stage (Figure 3-5 F). Several inflammation-related proteins (C1qc, Ilf2, Igh-3) and components of the myelin sheath (Mag, Mog) were also identified to be strongly up- and downregulated at the preterminal stage of the disease which hints towards progressive inflammation and demyelination in the $3 \times \mathrm{Tg}$-AD model (Figure 3-5 G and $\mathrm{H}$ ).

The full list of quantified proteins at each time point with corresponding $\log _{2} \mathrm{AD} /$ control ratios and p-values is presented in Supplementary table 1.

\subsubsection{Relating proteome changes to biological function}

To understand which biological processes were most affected at each disease stage, I applied $\begin{array}{llll}\text { ingenuity pathway analysis (IPA) (QIAGEN } & \end{array}$ https://www.qiagenbioinformatics.com/products/ingenuity-pathway-analysis) feeding $\log _{2} \mathrm{AD} /$ control ratio for each quantified protein to IPA as an input. IPA allows one to interpret "omics" data in a broader biological contest by relating protein or gene expression levels to biological function. The information on the relationship between certain protein or gene and the biological process, pathway or disease it is involved in is retrieved from the curated IPA Knowledge Base. The activation status of the biological function in the dataset can be then determined by the z-score which is calculated based on experimental protein expression data (in this case $\log _{2} \mathrm{AD} /$ control ratio) and the information stored in the IPA Knowledge Base. Positive z-score values indicate an activation of a biological pathway or function, while negative values indicate its inhibition. 

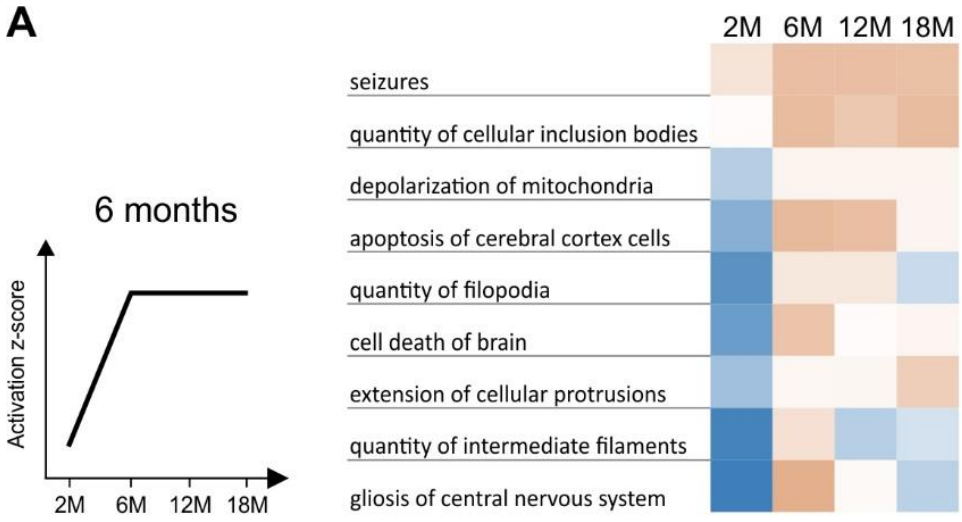

B
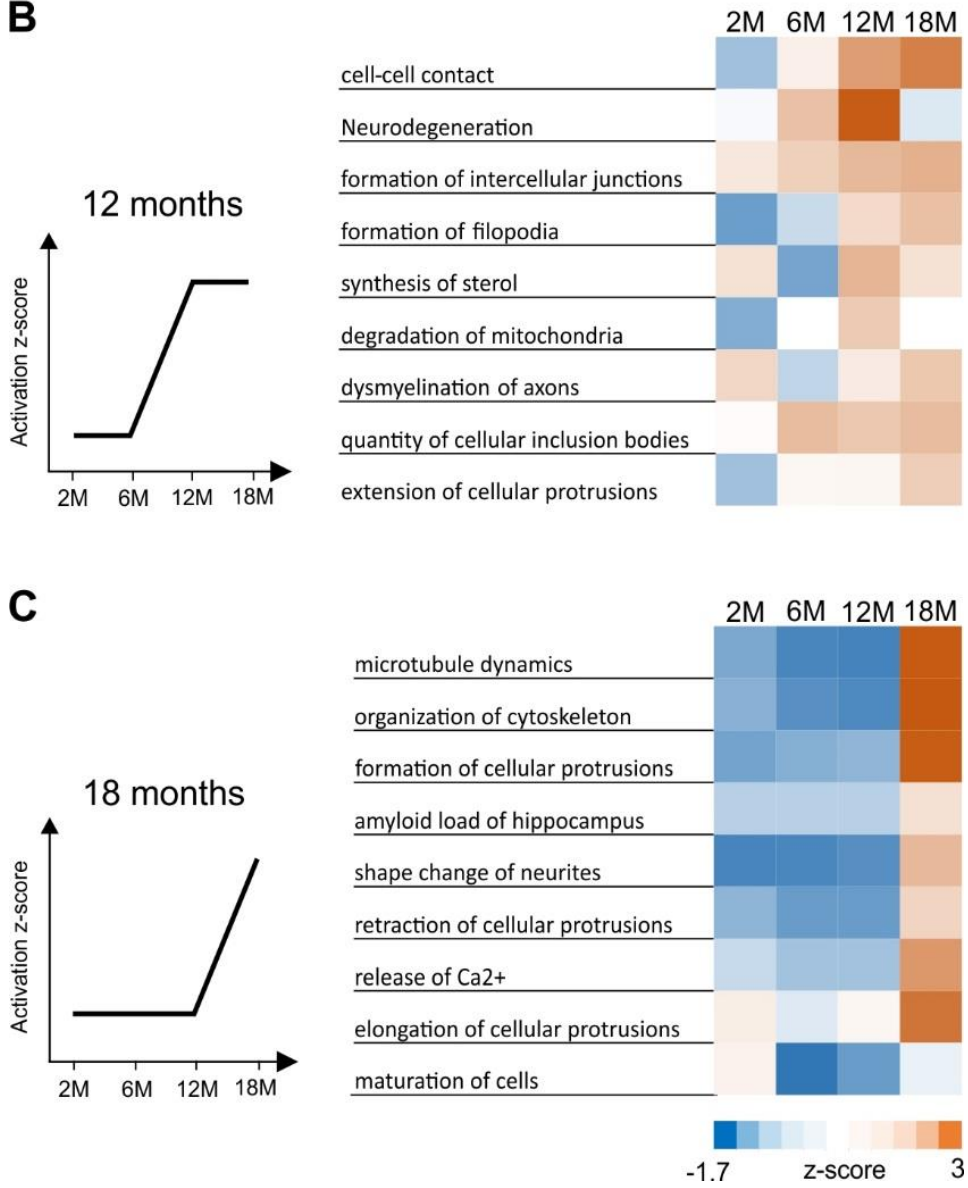

Figure 3-6. Inference of activated biological processes in the progression of $A D$ in $3 \times T g-A D$ mice.

(A, B, C) Activation of biological processes at different stages of the disease assessed by ingenuity pathway analysis (IPA). Heatmaps represent activation z-score change over the course of disease progression and indicate pathways that are activated at 6M (A), 12M (B), and 18M (C). 
The biological processes that were activated most at early (6 months, Figure 3-6 A), intermediate (12 months, Figure 3-6 B) and preterminal (18 months, Figure 3-6 C) symptomatic stages of the disease were ranked by their z-score trend. Remarkably, a cumulative upregulation in expression of proteins involved in cell death and apoptotic processes could already be detected at the first symptomatic time point (6 months) (Figure 3-6 A). Significant changes in regulation of proteins associated with seizures was also observed at the transition point between the presymptomatic phase and 6 months which corresponds to the stage where changes in LTP in $3 \times \mathrm{Tg}-\mathrm{AD}$ mice were previously described ${ }^{233,296}$. Neurodegeneration-related processes (amyloid load of hippocampus, demyelination of axons and degradation of mitochondria) were also noticeably exacerbated with AD progression (Figure 3-6 B and C). Notably, the disturbance of the cytoskeleton, which is a hallmark of many neurodegenerative disorders including AD, became prominent only at the late stages of the disease (18 months) (Figure 3-6 C) which coincides with the timeline of tau aggregation in $3 \times \mathrm{Tg}-\mathrm{AD}$ mice. Interestingly, IPA analysis revealed that the protein signatures of dysfunctional mitochondria were among the very first signs of an altered AD proteome (Figure 3-6 A and B).

I further used IPA to identify the upstream master regulators of the $3 \times \mathrm{Tg}-\mathrm{AD}$ proteome. This module of IPA determines the most activated/inhibited regulators of protein expression based on the expression levels of their downstream targets. As expected, tau (MAPT), APP and PSEN1 were among the top activated upstream regulators (Figure 3-7 A) at each analyzed time point. This result serves as a robust internal control indicating that these three genes contribute most to the change of protein expression between $3 \times \mathrm{Tg}-\mathrm{AD}$ and control mice. Additionally, proteins located downstream of tau, APP and PSEN1 in our dataset largely overlap (Figure 3-7 B). Interestingly, functional enrichment indicates that these downstream effectors of tau, APP and PSEN1 are mainly involved in biological processes such as apoptosis, mitochondria dysfunction and oxidative stress (Figure 3-7 C).

Overall, the analysis of the brain proteome demonstrates progressive stage-dependent differences in protein expression between $3 \times \mathrm{Tg}-\mathrm{AD}$ and control mice. Based on these data, I could determine a timeline of biological processes dysregulated along AD progression. In section 3.3, I will focus specifically on proteins whose expression was significantly affected at the presymptomatic stage of the disease. 
A

\begin{tabular}{|l|l|l|l|l|}
\hline \multirow{2}{*}{\multicolumn{1}{c|}{ Top 5 upstream regulators }} & \multicolumn{4}{c|}{ p-value } \\
\cline { 2 - 5 } & \multicolumn{1}{c|}{$\mathbf{2 M}$} & $\mathbf{6 M}$ & \multicolumn{1}{c|}{$12 \mathrm{M}$} & \multicolumn{1}{c|}{$18 \mathrm{M}$} \\
\hline Microtubule associated protein tau (MAPT) & $5.54 \mathrm{E}-54$ & $1.19 \mathrm{E}-54$ & $1.50 \mathrm{E}-53$ & $2.59 \mathrm{E}-50$ \\
\hline Amyloid beta precursor protein (APP) & $5.84 \mathrm{E}-37$ & $6.87 \mathrm{E}-38$ & $1.62 \mathrm{E}-37$ & $6.52 \mathrm{E}-34$ \\
\hline Presenilin 1 (PSEN1) & $3.17 \mathrm{E}-26$ & $9.77 \mathrm{E}-27$ & $5.76 \mathrm{E}-26$ & $7.63 \mathrm{E}-26$ \\
\hline Mechanistic target of rapamycin kinase (MTOR) & $4.05 \mathrm{E}-21$ & $1.02 \mathrm{E}-21$ & $2.26 \mathrm{E}-22$ & $2.56 \mathrm{E}-22$ \\
\hline MAP kinase interacting serine/threonine kinase 1 (MKNK1) & $2.09 \mathrm{E}-20$ & $4.61 \mathrm{E}-21$ & $1.25 \mathrm{E}-20$ & $8.50 \mathrm{E}-21$ \\
\hline
\end{tabular}

B

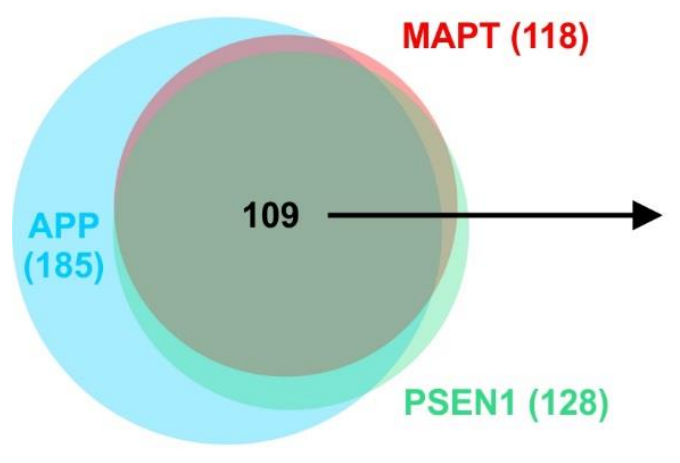

C

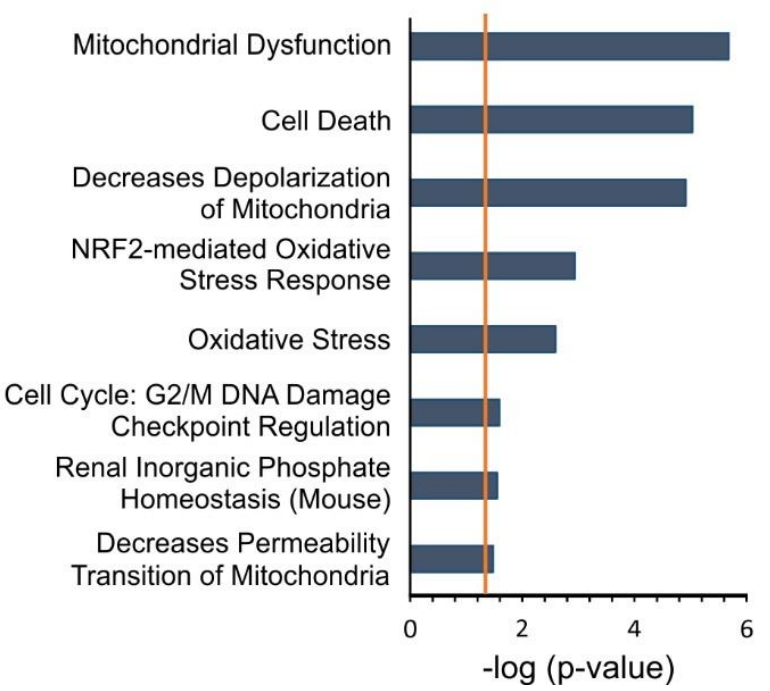

Figure 3-7. Identification of top upstream regulators in the proteomic dataset

(A) Top5 upstream regulators identified by ingenuity pathway analysis (IPA) of the complete proteome dataset $\left(\log _{2} \mathrm{AD} /\right.$ control) at each time point. MAPT (tau), APP and PSEN1 were identified as the top 3 upstream regulators. (B) Regulated proteins downstream of MAPT, APP and PSEN1 largely overlap (109). (C) Enrichment analysis (IPA) of overlapping proteins reveals their strong association with mitochondrial dysfunction, cell death and oxidative stress. Presented protein enrichment data are for the time point 18 months. The orange line indicates the p-value cut-off, $\mathrm{p}<0.05$.

\subsection{Alterations of brain phosphoproteome in $A D$ progression}

In the next step of my thesis, I aimed to analyze the brain phosphoproteome and determine how the protein phosphorylation profile of the brain changes during AD progression. Phosphopeptides compose a relatively small subpopulation of peptides in the entire brain proteome and therefore their detection by shotgun proteomics would be masked by more abundant peptide species. To overcome this problem and to increase the identification of phosphorylated peptides, phosphopeptide enrichment using titanium dioxide beads was 
performed. The phosphopeptide-enriched fraction was then analyzed by label free LC/MSMS.

A

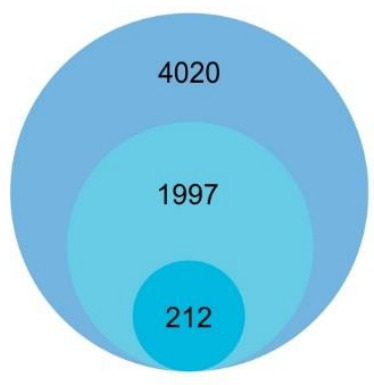

Identified phosphosites

Quantified phosphosites

Regulated phosphosites at least in one time point
B
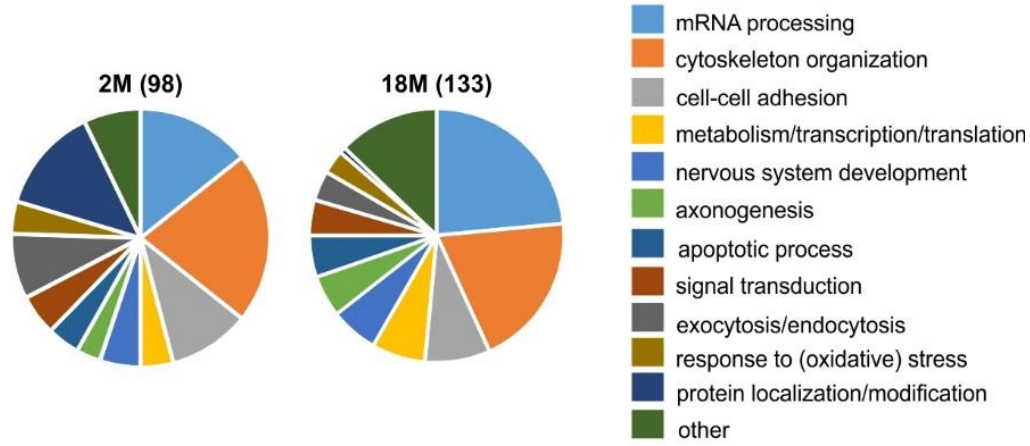

Figure 3-8. Analysis of brain phosphoproteome at presymptomatic and late stages of $\mathrm{AD}$ in $3 \times \mathrm{Tg}$-AD mice.

(A) Overview of identified, quantified and regulated phosphosites at time point 2 and 18 months. Phosphosites statistically different between $3 \times \mathrm{Tg}$ - $\mathrm{AD}$ and control mice ( $\mathrm{t}$-test, $\mathrm{p}$-value $<0.05$ ) and with an expression change of at least 50\% were considered as "regulated". (B) Functional classification of proteins with at least one significantly regulated phosphosite at 2 and 18 months. Classification was performed based on the Gene Ontology Biological Function database.

Data for these figure were prepared in collaboration with Dr. Mahdokht Kohansal-Nodehi (Department of Neurobiology).

The soluble brain fraction obtained from 2- and 18-month old mice was used for phosphoproteomic analysis to focus specifically at the differences between the presymptomatic and the late symptomatic time points. A total of 4020 phosphosites were identified at these two time points (Figure 3-8 A).

To reflect the changes in phosphosite levels only, the quantified $\log _{2}$ ratio $\mathrm{AD} /$ control for each individual phosphosite was normalized to the same ratio of corresponding protein from the global proteome datase ${ }^{297}$. The resulting phospho/global ratio accounts for the difference of the phosphopeptide levels between $3 \times \mathrm{Tg}-\mathrm{AD}$ and control mice and is normalized to the total level of the protein to which it belongs. In this way, 1997 phosphosites were quantified at time points 2 and 18 months. The phosphopeptides that exhibited a statistically significant change (t-test, p-value $<0.05$ ) and at least a $50 \%$ change in comparison to the control were considered as "regulated phosphosites" and were assessed in further bioinformatics analyses. 
To determine the biological functions affected most by altered protein phosphorylation, proteins that exhibited regulated phosphosites in the dataset were classified according to the Gene Ontology database (Figure 3-8 B). Interestingly, at both presymptomatic and late stages of the disease, cytoskeletal proteins contributed to about $20 \%$ of regulated phosphosites. This is not surprising, given the known role of hyperphosphorylated tau in AD. I indeed identified multiple phosphosites of tau to be upregulated, some of them already at the 2 month time point (Supplementary table 2). Apart from tau, a number of other microtubule associated proteins (MAP1, MAP2, MAP7) demonstrated an altered phosphorylation profile. Extensive phosphorylation of neurofilaments was also detected in $3 \times \mathrm{Tg}-\mathrm{AD}$ mice with few phosphosites upregulated already at the presymptomatic stage of the disease.

A particularly interesting group of regulated phosphosites belong to the proteins involved in mRNA processing. Intriguingly, the number of regulated phosphosites of this group increased considerably between time point 2 and 18 months and comprised almost $25 \%$ of all regulated phosphosites at the preterminal stage. This result correlates well with the data obtained in proteomic analysis, where proteins involved in mRNA processing demonstrated steadily increasing expression with $\mathrm{AD}$ progression. Most of the phosphosites in this group belong to just two proteins: serine/arginine rich motif protein 1 and 2 (SRRM1-2) (Figure 3-9). SRRM proteins play a role in alternative splicing298, however, it is not known how their phosphorylation contributes to this process.

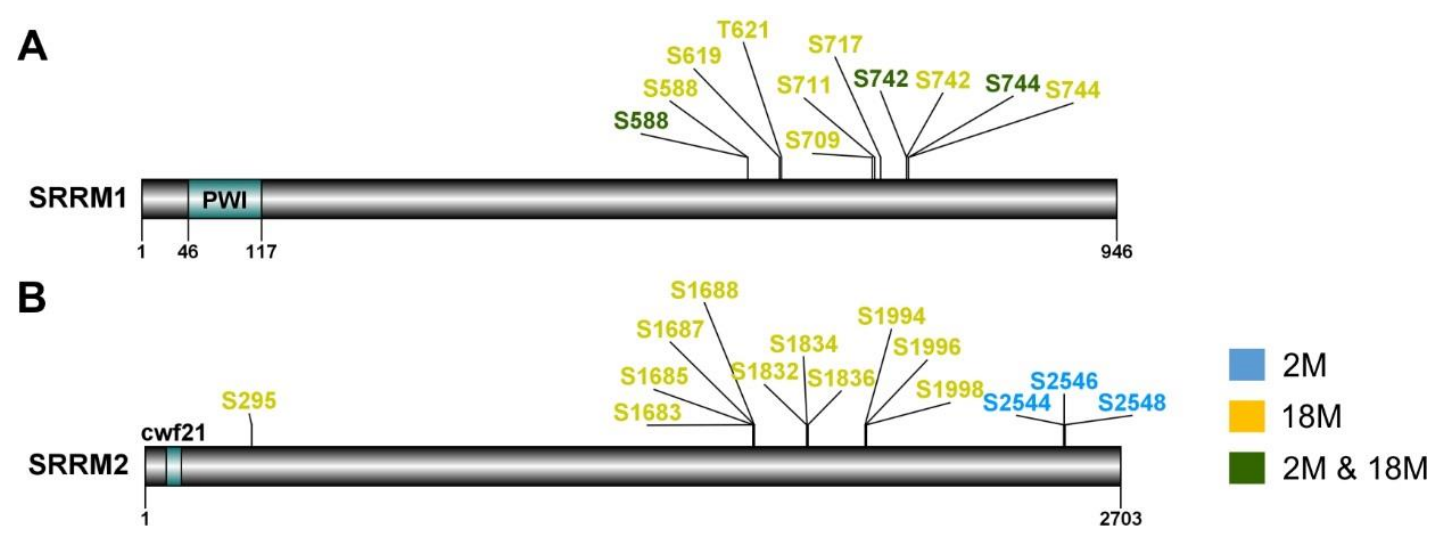

Figure 3-9. SRRM1 and 2 are phosphorylated at numerous phosphosites in the late stage of AD in $3 \times \mathrm{Tg}$ AD mice.

Upregulated phosphosites of SRRM1 and 2 at time point 2 months (blue), 18 months (yellow) or both time points (green). The protein diagrams were generated using DOG Illustrator ${ }^{299}$.

Data for these figure were prepared in collaboration with Dr. Mahdokht Kohansal-Nodehi (Department of Neurobiology).

Results $\mid 72$ 
Phosphorylation of proteins involved in formation of cell-to-cell contact, axogenesis, apoptosis and several other biological processes were also identified to be altered in the dataset. A substantial number of these changes could be detected already at the time point 2 months indicating that aberrations in protein phosphorylation and presumably protein function precede manifestation of AD symptoms in 3×Tg-AD mice.

A

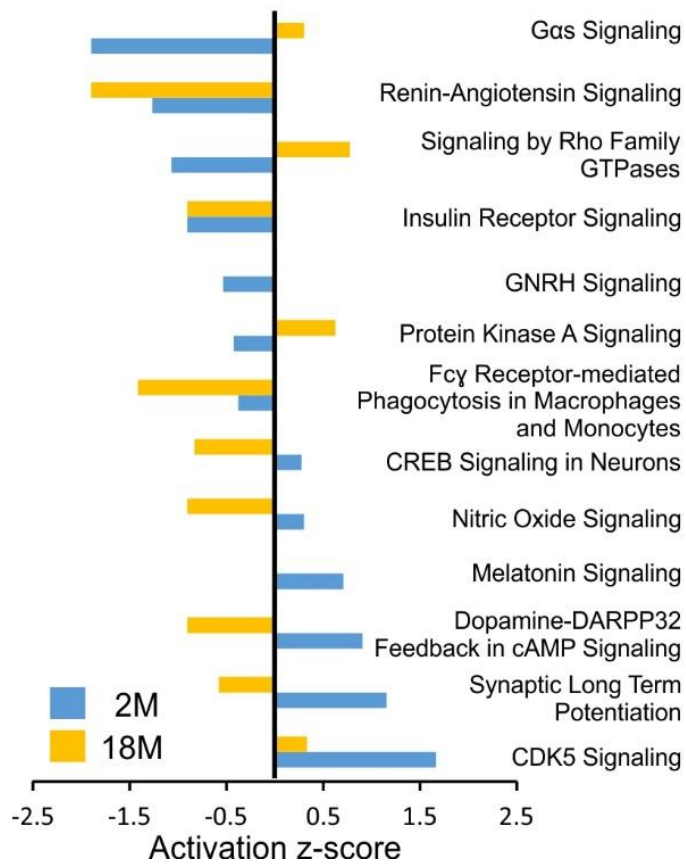

B

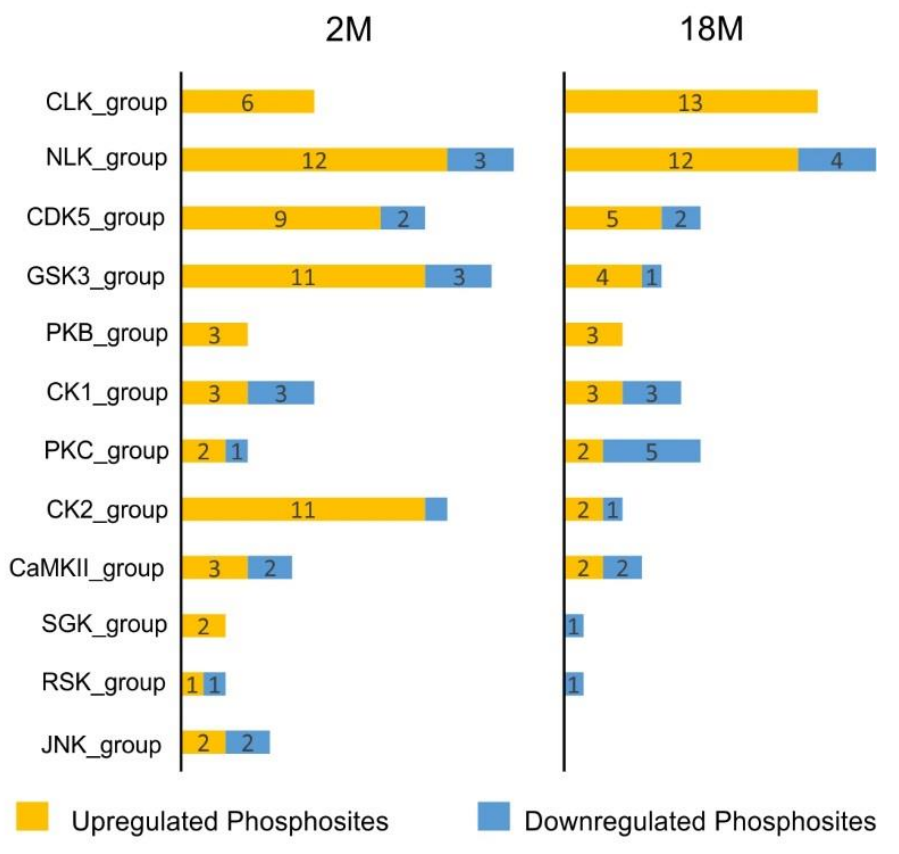

Figure 3-10. Canonical signaling pathways and kinase families affected in $3 \times \mathrm{Tg}-\mathrm{AD}$ at the presymptomatic and late stage of the disorder.

(C) Enriched signaling pathways in the phosphoproteome dataset at 2 and 18 months (determined by IPA). The positive and negative values of the z-score correspond to activated and deactivated signaling pathways, respectively. (D) Kinase groups predicted to phosphorylate up- (yellow) and downregulated (blue) phosphosites in the dataset were determined by a kinase prediction tool (NetworKIN, version 3.0282).

Data for these figure were prepared in collaboration with Dr. Mahdokht Kohansal-Nodehi (Department of Neurobiology).

Protein phosphorylation is a key event in many signaling cascades. To gain knowledge of specific pathways that are affected in early and late stages of AD, IPA was applied to determine regulated canonical signaling pathways by activation z-score (Figure 3-10 A). Consistent with previous findings, the CREB pathway and nitric oxide (NO) signaling were found to be downregulated in $3 \times \mathrm{Tg}-\mathrm{AD}$ mice at the late stage of the disease 300,301 . Mixed results were reported with regard to the role of the PKA cascade in $\mathrm{AD}^{302,303}$. In my analysis, PKA signaling was found to be slightly activated at a time point 18 months. Interestingly, 
several signaling pathways demonstrated considerable alterations already at the presymptomatic stage. For example, signaling through G-protein subunit $\alpha(\mathrm{G} \alpha \mathrm{S})$ was significantly downregulated at time point 2 months while it demonstrated little change in the late stage of the disease. CDK5 signaling was strongly upregulated in $3 \times \mathrm{Tg}$-AD mice at the presymptomatic time point. CDK5 is known for its phosphorylation of tau, neurofilaments and APP and its hyperactivation in AD was previously reported ${ }^{304}$. Interestingly, the observed activation of the CDK5 pathway was much more prominent at the presymptomatic stage than at late stage of the disease. This result indicates that CDK5 hyperactivity preceded symptomatic onset in the $3 \times$ Tg-AD mouse model and might be one of the early events happening in $\mathrm{AD}$.

In addition to the analysis of canonical signaling pathways involved in $A D$, the information on individual kinase families was extracted from the phosphoproteomics dataset (Figure 3$10 \mathrm{~B})$. To this end, the kinases responsible for phosphorylation of regulated phosphosites identified in our study were predicted using a kinase prediction tool (NetworKIN, version $3.0^{282}$ ). This analysis revealed that the substrates of GSK3 and CDK5 kinases demonstrated increased phosphorylation in $3 \times \mathrm{Tg}-\mathrm{AD}$ mice. Most of the phosphosites predicted to be phosphorylated by GSK3 and CDK5 in my dataset belong to cytoskeletal proteins. Interestingly, tau was primarily phosphorylated by GSK3, while CDK5 was mainly responsible for phosphorylation of MAP1 and neurofilaments. In line with the data obtained on canonical signaling pathways, higher number of upregulated phosphosites of the CDK5 kinase family was observed at the time point 2 months. A similar profile was obtained for CK2 kinase. Among upregulated CK2 substrates, MAP1, calcineurin and the microtubulebinding protein stathmin were identified. These data suggest that abnormal activity of kinases related to microtubule organization occurs early in the disease progression.

A very interesting kinase family that demonstrated an age-dependent difference in substrate phosphorylation in 3×Tg-AD mice was a group of Cdc2-like kinases (CLKs). CLKs localize to nucleus and are known to phosphorylate a wide variety of nuclear matrix proteins including members of the serine/arginine-rich (SR) family of splicing factors ${ }^{305}$. The previously mentioned SRRM1-2 proteins that were heavily phosphorylated at time point 18 months belong to this group. An increased number of upregulated CLK phosphosites at the preterminal stage additionally strengthens the observation of a concomitant increase in Results $\mid 74$ 
expression and phosphorylation of proteins involved in mRNA processing with the progression of $\mathrm{AD}$ in $3 \times \mathrm{Tg}-\mathrm{AD}$ mice.

\subsection{Putative presymptomatic markers of Alzheimer's disease}

\subsubsection{Identification of proteins involved in early stage of $A D$}

In the next step of my thesis, I returned to the proteomic data to focus specifically on the proteins that demonstrated the strongest degree of change at the presymptomatic stage. The knowledge of proteins whose expression is dysregulated early on in the disease progression is important for two main reasons. First, they can serve as potential early markers of the disease onset. Second, these proteins might be responsible for the initial pathogenic events, thus deciphering their function can help us better understand through which mechanisms $\mathrm{AD}$ is driven in the first place.

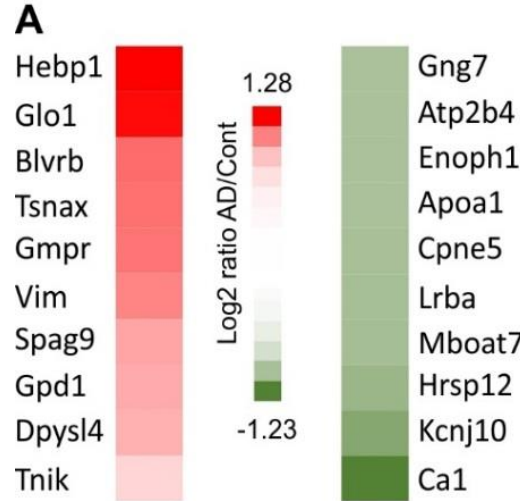

B
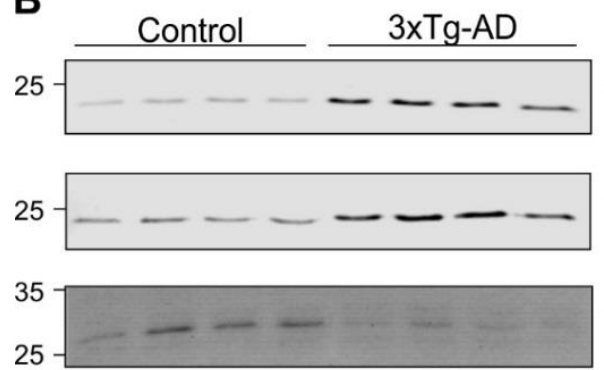

55

5

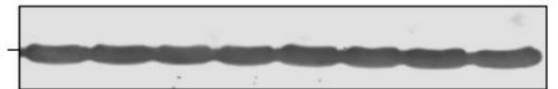

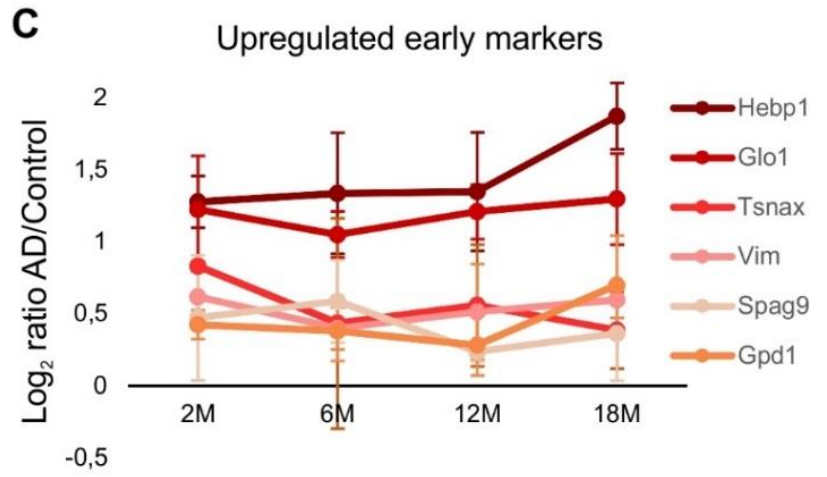
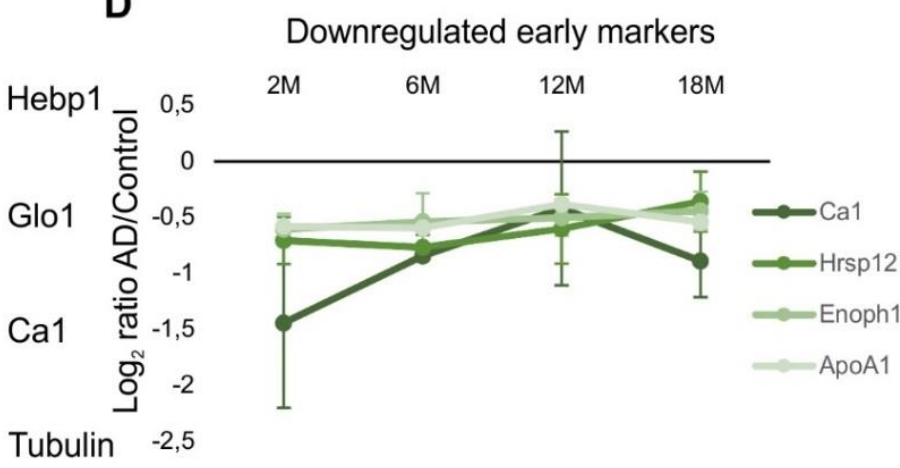

Figure 3-11. Putative early markers of Alzheimer's disease.

(A) Top 10 significantly up- and downregulated proteins in $3 \times$ Tg-AD mice at presymptomatic time point (2 months). (B) Immunoblotting analysis of most regulated hits: heme-binding protein 1 (Hebp1), glyoxalase 1 (Glo1) and carbonic anhydrase 1 (CA1). $\alpha$-tubulin was used as a loading control. Presymptomatic markers that remain up- (C) or downregulated (D) across all time points of AD progression in $3 \times \mathrm{Tg}-\mathrm{AD}$ mice. 
Table 3-1. Putative presymptomatic brain markers of AD in literature.

\begin{tabular}{|c|c|c|c|c|}
\hline Gene name & Protein name & $\begin{array}{l}\log _{2} \\
\mathrm{AD} / \mathrm{Ctrl}\end{array}$ & $\begin{array}{l}\text { Previous } \\
\text { involvement in AD }\end{array}$ & Reference \\
\hline \multicolumn{5}{|c|}{ Upregulated early markers } \\
\hline Hebp1 & Heme-binding protein 1 & 1,28 & - & - \\
\hline Glo1 & Glyoxalase 1 & 1,24 & $\begin{array}{l}\uparrow \text { in human brain, } \\
\text { mouse model of FTD }\end{array}$ & $\begin{array}{l}\text { Chen et al. } \\
2004^{306}, \text { More } \\
\text { et al., } 2013^{307}\end{array}$ \\
\hline Blvrb & Biliverdin Reductase B & 0,74 & $\uparrow$ in plasma & $\begin{array}{l}\text { Mueller et al., } \\
2010^{308}\end{array}$ \\
\hline Tsnax & Translin Associated Factor X & 0,72 & - & - \\
\hline Gmpr & $\begin{array}{l}\text { Guanosine Monophosphate } \\
\text { Reductase }\end{array}$ & 0,70 & $\begin{array}{l}\uparrow \text { human brain, early } \\
\text { stage }\end{array}$ & $\begin{array}{l}\text { Liu et al, } \\
2018^{309}\end{array}$ \\
\hline Vim & Vimentin & 0,62 & $\begin{array}{l}\uparrow \text { in human brain } \\
\text { (astrocytes) }\end{array}$ & $\begin{array}{l}\text { Yamada et al., } \\
1992^{310}\end{array}$ \\
\hline Spag9 & Sperm Associated Antigen 9 & 0,46 & - & - \\
\hline Gpd1 & $\begin{array}{l}\text { Glycerol-3-Phosphate } \\
\text { Dehydrogenase } 1\end{array}$ & 0,43 & Accumulation in NFT & $\begin{array}{l}\text { Wang et al., } \\
2005^{311}\end{array}$ \\
\hline Dpysl4 & Dihydropyrimidinase Like 4 & 0,40 & - & - \\
\hline Tnik & $\begin{array}{l}\text { TRAF2 and NCK Interacting } \\
\text { Kinase }\end{array}$ & 0,22 & $\begin{array}{l}\text { Accumulation in } \\
\text { insoluble fraction of } \\
\text { amygdala in } \\
\text { cognitively impaired } \\
\text { patients }\end{array}$ & $\begin{array}{l}\text { Gal et al., } \\
2017312\end{array}$ \\
\hline \multicolumn{5}{|c|}{ Downregulated early markers } \\
\hline Gng7 & G Protein Subunit Gamma 7 & $-0,60$ & - & - \\
\hline Atp2b4 & $\begin{array}{l}\text { ATPase Plasma Membrane } \\
\mathrm{Ca}^{2+} \text { Transporting } 4\end{array}$ & $-0,60$ & $\downarrow$ in human brain & $\begin{array}{l}\text { Kong et al., } \\
2015^{313}\end{array}$ \\
\hline Enoph1 & Enolase-Phosphatase 1 & $-0,60$ & - & - \\
\hline Apoa1 & Apolipoprotein A1 & $-0,60$ & $\downarrow$ in plasma & $\begin{array}{l}\text { Saczynski et } \\
\text { al., } 2007314 \text {, } \\
\text { Merched et al., } \\
2000^{315}\end{array}$ \\
\hline Cpne5 & Copine 5 & $-0,62$ & - & - \\
\hline Lrba & $\begin{array}{l}\text { LPS Responsive Beige-Like } \\
\text { Anchor Protein } \\
\text { Membrane Bound O- }\end{array}$ & $-0,63$ & - & - \\
\hline Mboat7 & $\begin{array}{l}\text { Acyltransferase Domain } \\
\text { Containing } 7\end{array}$ & $-0,63$ & - & - \\
\hline Hrsp12 & Ribonuclease UK114 & $-0,71$ & $\uparrow$ in CVN-AD model & $\begin{array}{l}\text { Hoos et al., } \\
2013^{316}\end{array}$ \\
\hline Kcnj10 & $\begin{array}{l}\text { Potassium Voltage-Gated } \\
\text { Channel Subfamily J Member } \\
10\end{array}$ & $-0,86$ & $\begin{array}{l}\downarrow \text { in mouse model of } \\
\text { ALS }\end{array}$ & $\begin{array}{l}\text { Kaiser M et al., } \\
2006^{317}\end{array}$ \\
\hline Ca1 & Carbonic anhydrase 1 & $-1,23$ & - & - \\
\hline
\end{tabular}


I identified the proteins that were strongly up- or downregulated at the time point 2 months (Figure 3-11 A) and corroborated the most prominent hits by immunoblotting (Figure 3-11 B). Many of the early markers remained over or under-expressed in 3×Tg-AD mice across all time points suggesting their importance for the late stages of $\mathrm{AD}$ as well (Figure 3-11 C and D).

Noteworthy, among the identified early brain markers of $A D$, several proteins were previously reported for their involvement in AD or other neurodegenerative disorders and followed the same direction of expression change (Table 3-1). For example, decreased levels of ApoA1 that are observed in this dataset have been linked to the increased severity of AD in human patients ${ }^{314,315}$. Recently, guanosine monophosphate reductase 1 (Gmpr) was identified to be expressed at higher levels in the brain of AD patients ${ }^{309}$. Elevated expression of glyoxalase-1 (Glo1) was also shown in other mouse models of neurodegeneration ${ }^{306}$ and restoration of its activity has been proposed as a mechanism to combat cognitive dysfunction in $\mathrm{AD}^{307}$.

\subsubsection{Expression of identified presymptomatic markers in postmortem brain samples from $A D$ patients}

To verify the relevance of identified presymptomatic markers in the mouse model for the disease in humans, I examined the expression of Heme-binding protein 1 (Hebp1) and glyoxalase 1 (Glo1), two most prominent early markers, in the postmortem brain samples obtained from AD patients and age-matched healthy controls (Table 2-10). I could confirm an overall higher expression of both proteins in $\mathrm{AD}$ patients compared to controls that validates the relevance of the findings in the 3×Tg-AD model (Figure 3-12 A). Interestingly, a strong difference in expression of Hebp1 and Glo1 was only observed in the subset of rapidlyprogressing cases (death within 4-year period after diagnosis) (Figure 3-12 B and C). The rapidly-progressing forms of $\mathrm{AD}$ are characterized by distinct pathological features and clinical parameters and are at large associated with a more severe and faster progression of the disease 318,319 . 

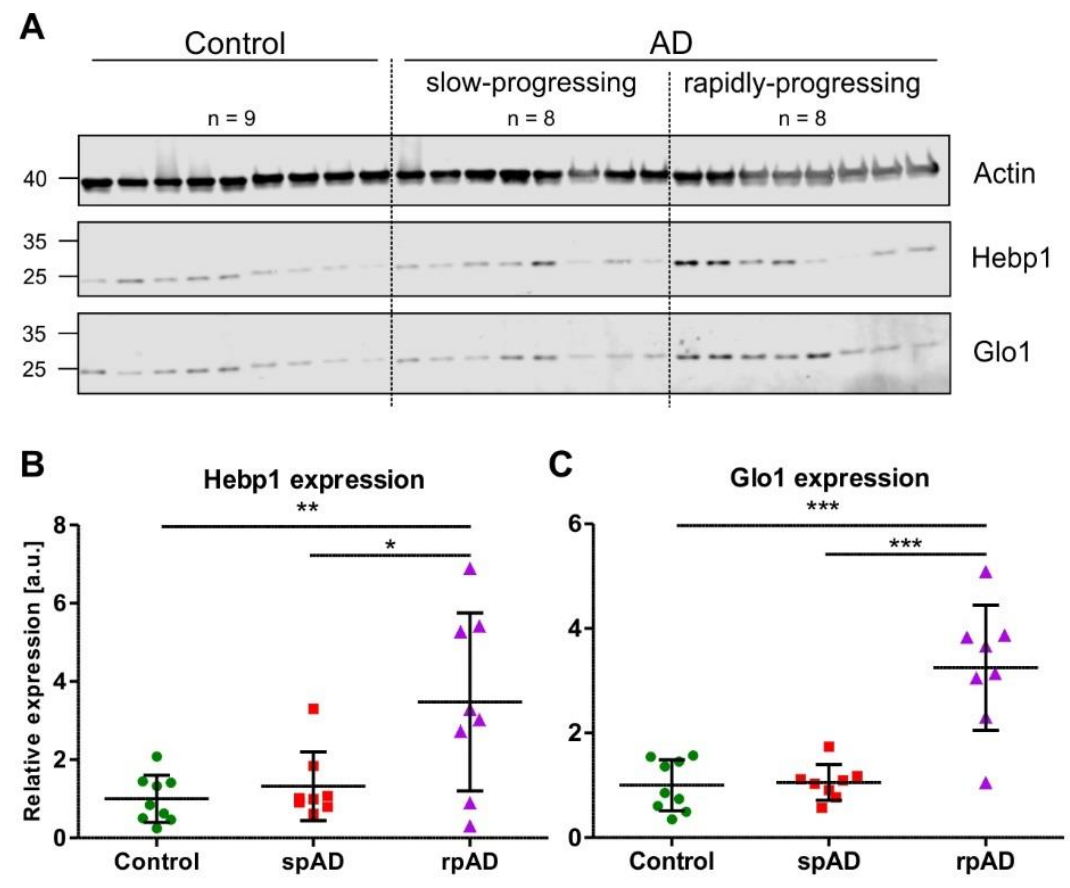

Figure 3-12. Hebp1 and Glo1, identified presymptomatic markers in 3×Tg-AD model, exhibit increased expression in brains of patients with rapidly-progressing forms of AD.

(A) Immunoblotting analysis of Hebp1 and Glo1 expression in slowly-progressing (spAD) and rapidlyprogressing (rpAD) AD cases and age-matched controls. Quantification of Hebp1 (B) and Glo1 (C) levels in human samples. Graphs represent mean \pm SD. Statistical significance in the datasets was assessed by one-way ANOVA followed by Student's t-test comparison for individual pairs of samples: ${ }^{*}$ p value $<0.05,{ }^{* *} p<0.01$, and ${ }^{* * *} \mathrm{p}<0.001$.

Hebp1 is a novel protein with regard to AD. To further validate its involvement in the disease, I have additionally examined the publicly available mRNA expression datasets to check the levels of Hebp1 in larger cohorts of AD patients (http://www.genenetwork.org/webqtl/main.py) (Figure 3-13). The datasets from Harvard Brain Tissue Resource Center (GN327 and GN328) demonstrated significantly increased levels of Hebp1 mRNA in the prefrontal and primary visual cortex in AD patients indicating an involvement of Hebp1 in AD in humans.

Half of the identified presymptomatic markers demonstrated no previous record of engagement in $\mathrm{AD}$ (Table 1-3). These novel markers provoke special interest due to their potential to explain the gaps in our understanding of $\mathrm{AD}$ pathogenesis and progression. 
A

Prefrontal cortex (HBTRC, GN328)

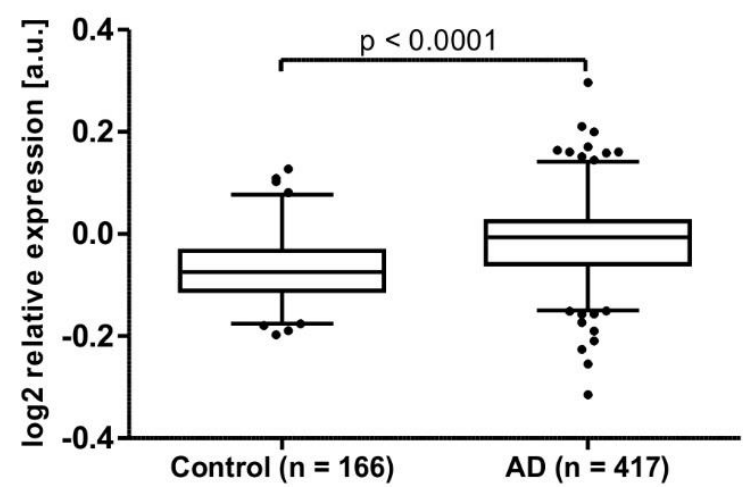

B

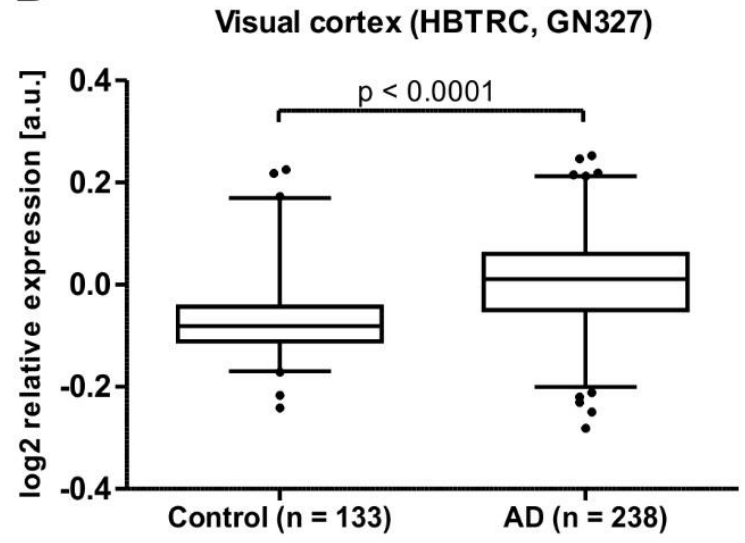

Figure 3-13. Hebp1 expression in publicly available transcriptome databases of Alzheimer's disease. In silico analysis of Hebp1 mRNA levels in AD patients and matched controls based on publicly available data from Harvard Brain Tissue Resource Center (HBTRC) deposited on GeneNetwork (www.genenetwork.org) (see Methods for details). Hebp1 expression in prefrontal cortex (A) and primary visual cortex (B) is elevated in AD patients. Box plot whiskers cover values from 2.5 to 97.5 percentile. Number of cases in each group is indicated in the graphs.

Among the newly identified putative presymptomatic markers of $\mathrm{AD}$, heme-binding protein 1 , was most consistently upregulated across all stages of the disease in $3 \times \mathrm{Tg}$ - $\mathrm{AD}$ mice. In agreement with this finding, expression of Hebp1 is significantly elevated in the brains of AD patients, particularly in rapidly-progressing cases. Overall, these data strongly suggest the implication of Hebp1 in Alzheimer's disease. In the next part of this thesis I focus specifically on Hebp1 and examine its function and potential role in AD by various biochemical and cell culture techniques.

\subsection{Investigation of heme-binding protein 1 and its potential functions in}

\section{AD pathogenesis}

Among the newly identified putative early markers of $\mathrm{AD}$, heme-binding protein 1 (Hebp1) is a particularly interesting candidate. Hebp1 belongs to the family of SOUL proteins and was originally identified as a tetrapyrrol-binding protein capable of binding protoporphyrin IX and heme ${ }^{320-322}$. To the best of my knowledge no information on Hebp1 function in the brain is available to date.

The ability of Hebp1 to bind heme poses special interest in context of AD pathology. Heme is essential for proper mitochondria functioning and cell survival ${ }^{323}$. Impairment of heme 
metabolism is also associated with $\mathrm{AD}^{147}$. Moreover, $\mathrm{A} \beta$ can form a complex with heme that reacts with oxygen and possesses strong peroxidase activity ${ }^{150-152}$. In this way, $A \beta$-heme complex can contribute largely to oxidative stress and cytotoxicity during AD. The proteomic analysis revealed that cell survival and mitochondria function might be among the first pathways affected in 3×Tg-AD mice (Figure 3-6 A-B). I thus further investigated the function of heme-binding protein 1 and its potential role in Alzheimer's disease.

\subsubsection{Hebp1 is a neuronal protein upregulated in the brain of $3 \times \mathrm{Tg}-\mathrm{AD}$ mouse}

To better understand the function of Hebp1 and how it contributes to the disease, I first examined its distribution in the brain. Immunoblotting analyses of four brain areas in 12month-old mice indicated that Hebp1 is most abundant in the hippocampus, followed by the brain stem and cortical areas. Hebp1 was not detectable in the cerebellum.

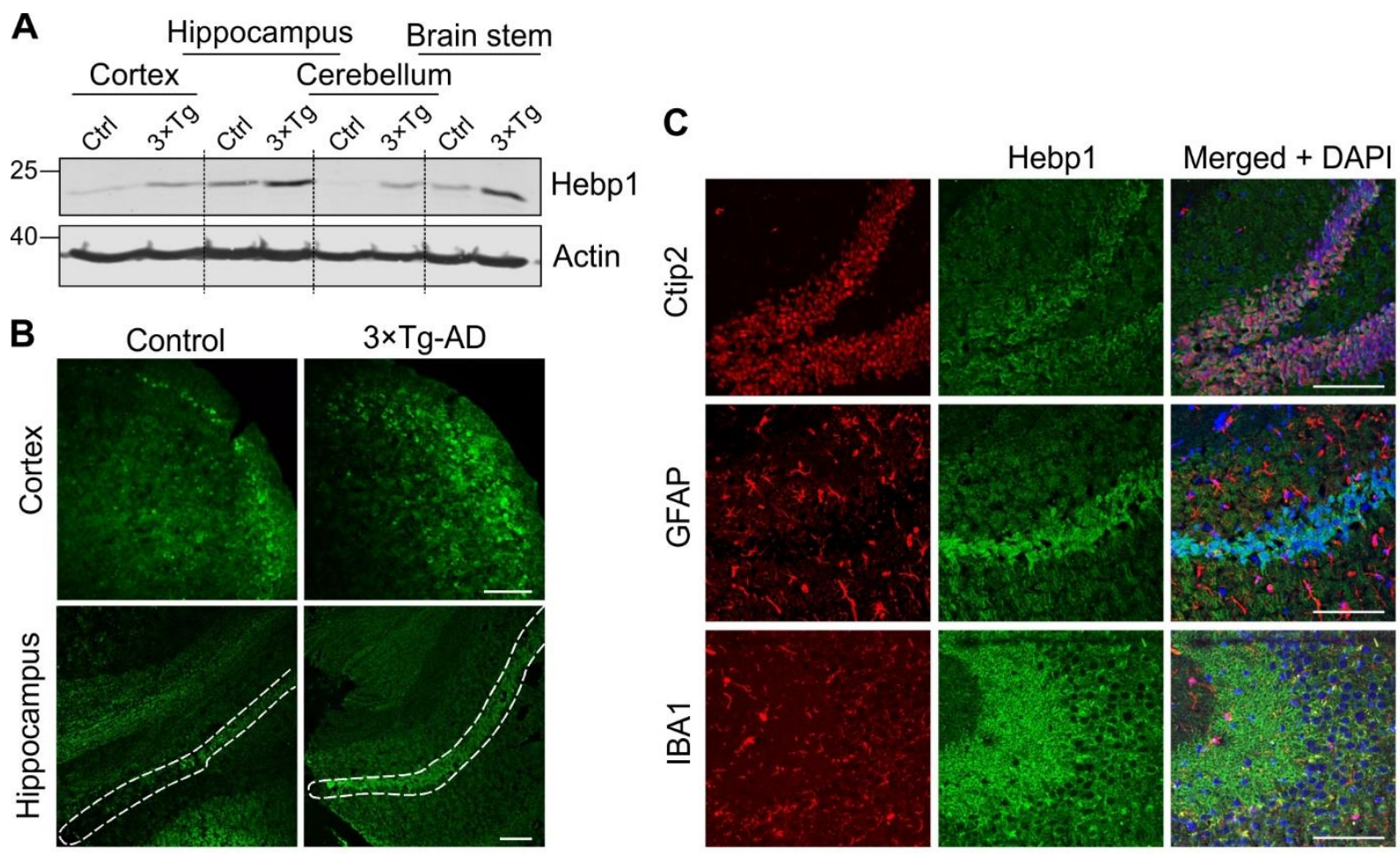

Figure 3-14. Analysis of Hebp1 expression in the brain of 3×Tg-AD mice.

(A) Expression of Hebp1 in 12-month-old control and $3 \times \mathrm{Tg}$-AD mice by brain region. (B) Hebp1 immunostaining of cortex and hippocampus (coronal sections). CA1 region is marked with the white dashed line. (C) Co-staining of Hebp1 with markers of CA1 and dentate gyrus neurons (Ctip2), astrocytes (GFAP) and microglia (IBA-1) in the hippocampus of $3 \times \mathrm{Tg}-\mathrm{AD}$ mice. Hepb1 is expressed predominantly in Ctip2-positive cells of the hippocampus (neurons). All images were acquired from 12-month-old control or $3 \times \mathrm{Tg}$ - $\mathrm{AD}$ mice. Scale bar is $100 \mu \mathrm{m}$.

Data for these figure were prepared in collaboration with Dr. Tamara Rabe (Department of Genes and Behavior)

Results $\mid 80$ 
Importantly, the levels of Hebp1 are dramatically elevated in all four brain regions in agematched 3×Tg-AD mice (Figure 3-14 A). Immunohistochemical analysis confirmed upregulated expression of Hebp1 in neocortex and hippocampus of 3×Tg-AD mice compared with wild type controls (Figure 3-14 B).

To identify cell types which express Hebp1 in the brain, I performed co-immunostaining of Hebp1 with cell-lineage specific markers (Figure 3-14 C). Hebp1 is strongly expressed in Ctip2-immunoreactive neurons but is poorly associated with GFAP-stained astrocytes or IBA-1-labeled microglia of hippocampus. These data indicate that Hebp1 is a neuronal protein and the increase in its expression in $3 \times \mathrm{Tg}-\mathrm{AD}$ mice is also attributed to neurons.

\subsubsection{Hebp1 interacts with the mitochondrial contact site complex}

The role of Hebp1 in neurons has not been characterized yet. Nevertheless, previous studies of Hebp1 and its homologues have left some clues regarding its potential function. First, Hebp1 can participate in the regulation of neuronal metabolism by transporting heme from mitochondria to the cytosol as was proposed previously320,321. Second, it might be involved in the cell response to cytotoxic stimuli. The homologue of Hebp1, heme-binding protein 2/SOUL, is known to mediate cell death by recruitment to mitochondria permeability transition pore 324,325 . Given the heme-binding properties of Hebp1 and its evolutionary similarity to Hebp2/SOUL ${ }^{326}$ I wanted to test whether Hebp1 is involved in one of these processes. In this case, mitochondrial or perimitochondrial localization of Hebp1 would be expected. Previous attempts to define the mitochondrial proteome led to mixed results with regard to mitochondrial localization of Hebp1327-329. To determine whether Hebp1 is associated with mitochondria, I performed subcellular fractionation of mouse brain and cultured HEK293 cells. In both experiments, Hebp1 is distributed equally in mitochondrial and soluble fractions, indicating that an appreciable portion of Hebp1 is indeed associated with mitochondria (Figure 3-15). 

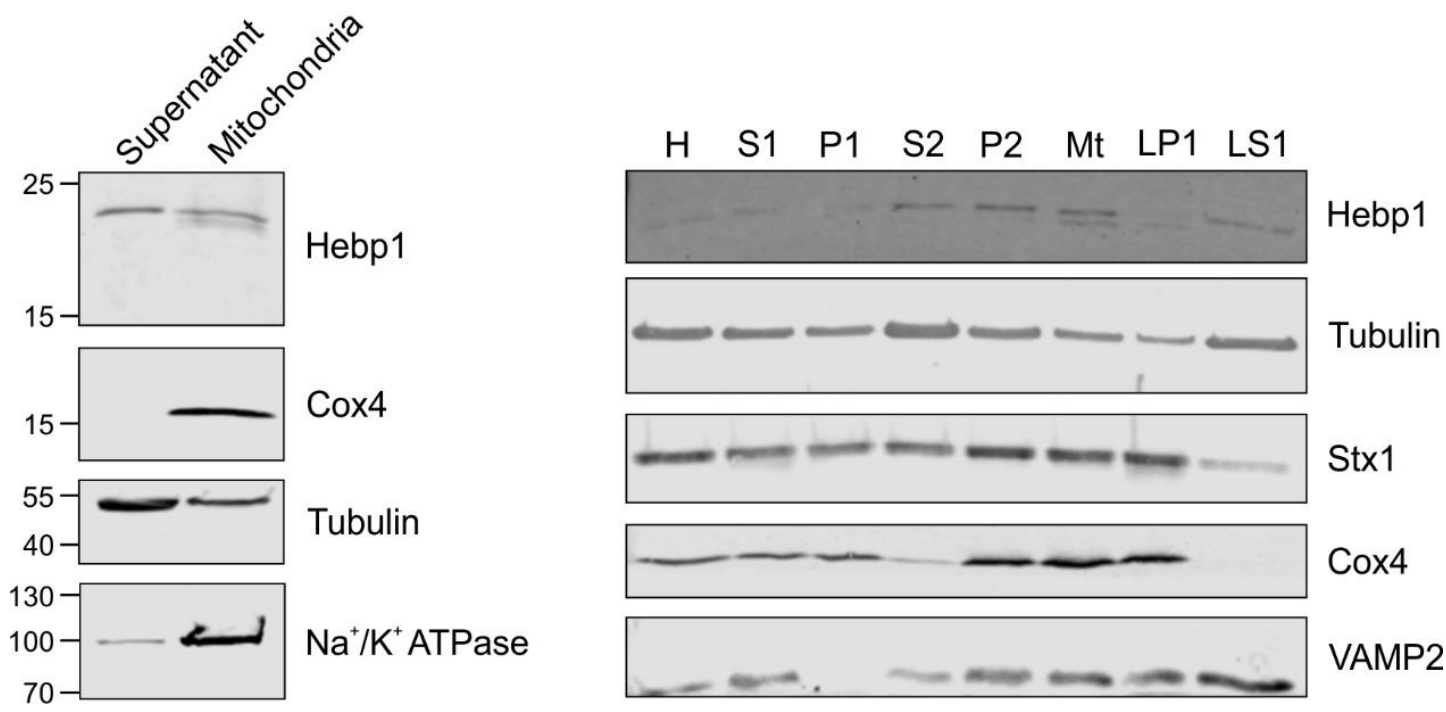

Figure 3-15. Subcellular localization of Hebp1.

(A) Subcellular fractionation of HEK293 cells. Hebp1 was enriched equally in supernatant (predominantly cytosol) and mitochondrial fractions. Presence of tubulin and $\mathrm{Na}^{+} / \mathrm{K}^{+}-\mathrm{ATPase}$ in mitochondria fraction indicates slight contamination with cytosolic and plasma membrane proteins, respectively.

(B) Brain fractionation was performed as described in Methods. Hebp1 was identified in crude mitochondria fraction (Mt). Fraction annotation: $\mathrm{H}$ - homogenate, S1 - supernatant 1, P1 - pellet 1, S2 - supernatant 2 (fraction of soluble proteins), P2 - pellet 2 (synaptosomes), Mt - mitochondria, LP1 - lysate pellet 1 (plasma membrane fraction of synaptosomes), LS1 - lysate supernatant 1 (soluble fraction of synaptosomes). $20 \mu \mathrm{g}$ of protein from each fraction were loaded on the gel, except for LS1 $-6 \mu \mathrm{g}$.

To test whether Hebp1 localizes inside the mitochondria I overexpressed Hebp1 tagged with EGFP in rat primary neurons and examined its co-localization with mitotracker by confocal microscopy (Figure 3-16). Remarkably, while Hebp1-EGFP and mitotracker signals demonstrated relatively low overlap, a large portion of Hebp1-EGFP puncta appeared in direct contact with mitochondria suggesting the perimitochondrial localization of Hebp1 (Figure 3-16).

I further examined the role of Hebp1 in neurons by identification of its binding partners. To this end, EGFP-tagged Hebp1 was overexpressed in primary cortical neurons and subsequently immunoprecipitated with a GFP-trap. Co-immunoprecipitated proteins were then investigated by MS analysis. The volcano plot represents the relative enrichment of detected proteins in either Hebp1-EGFP or EGFP (negative control) pull-downs (Figure 3-17 A). 
A

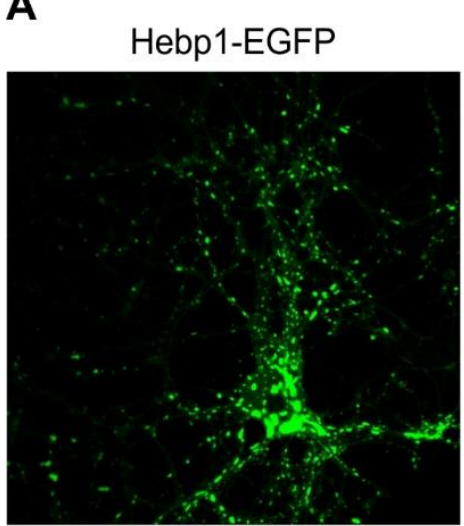

D

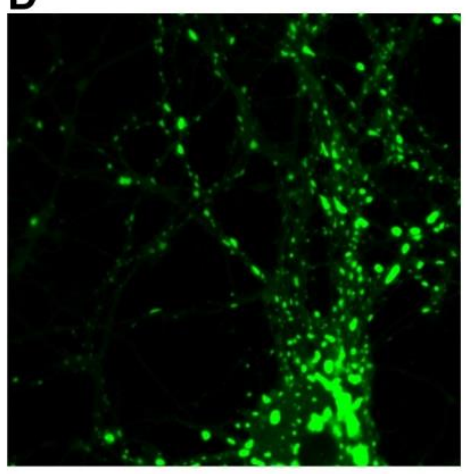

G

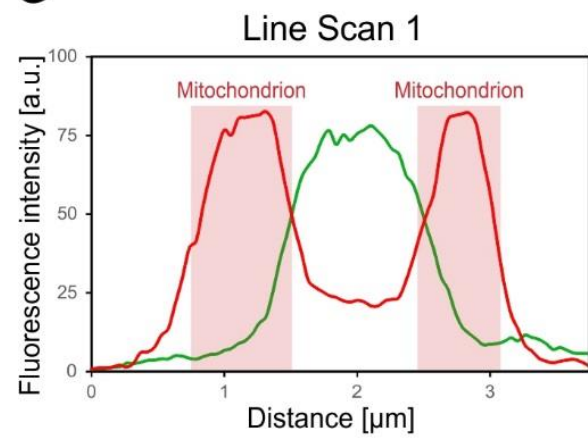

B

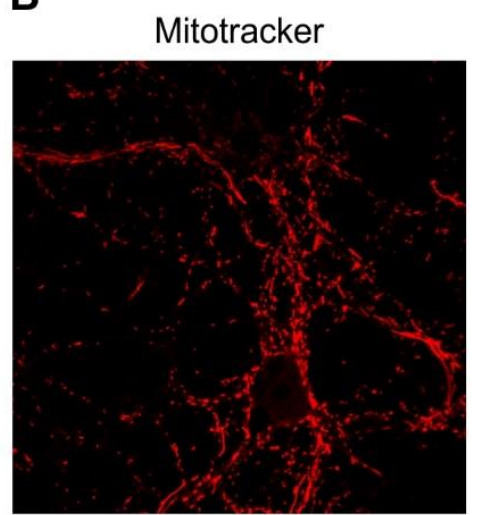

E

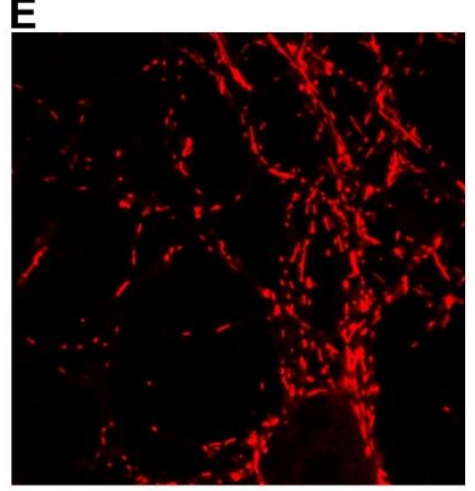

H

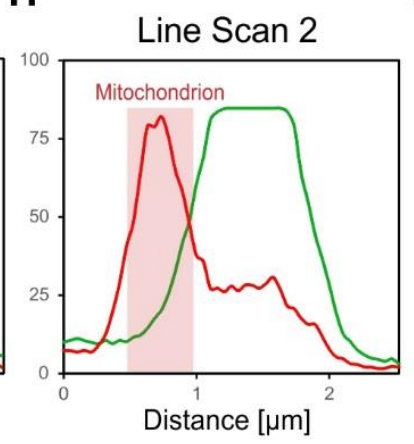

C

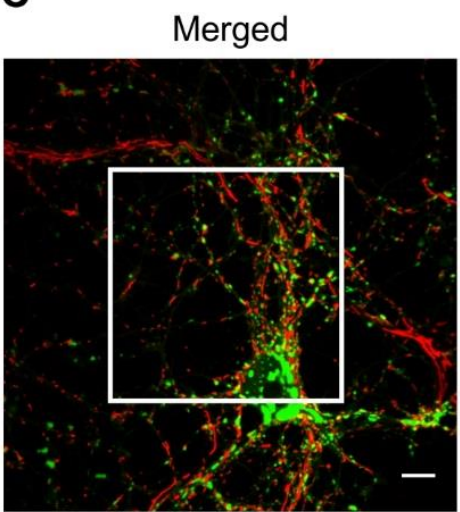

F

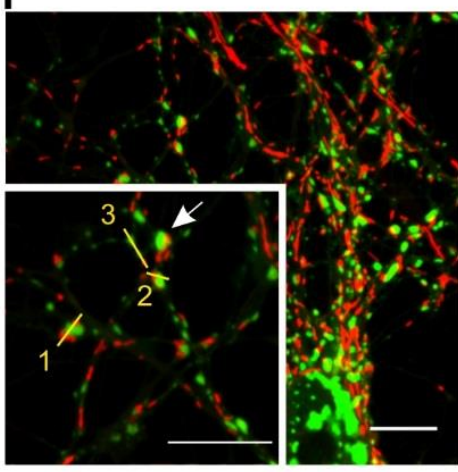

I

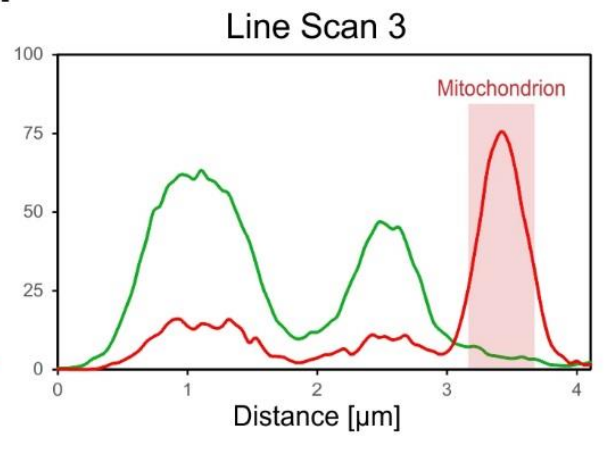

Figure 3-16. Hebp1 demonstrates perimitochondrial localization in cultured hippocampal neurons.

Analysis of Hebp1-EGFP co-localization with mitochondria (mitotracker) in cultured rat hippocampal neurons (DIV14) by laser scanning confocal microscopy. Low and high magnification images of Hebp1-EGFP (A, D), mitotracker CMXRos (B, E) or merged channels (C, F), respectively. Three representative line scans were obtained (G-I). Gold lines signed with the numbers at the starting point (F). Line scans demonstrate a direct contact between Hebp1-EGFP with mitochondria for some of the Hebp1-EGFP puncta (G,H). Scale bar is $10 \mu \mathrm{m}$.

Interestingly, the core components of the mitochondrial contact site and cristae organizing system (MICOS) complex, Mic60, Mic19 and Mic25, as well as proteins of the outer mitochondria membrane associated with MICOS complex, SAMM50 and Mtx2, were enriched in the Hebp1 Co-IP sample. I further validated the interaction between Hebp1 and Mic60 by immunoblotting (Figure 3-17 B). The observed interaction between Hebp1 and Mic60 was 
weak but specific. This result goes in line with the observations of co-localization between Hebp1 and mitochondria by confocal microscopy. Although Hebp1 puncta were in direct contact with mitochondria, most of the mitochondria were not associated with Hebp1. Therefore, it is reasonable to assume that only a small portion of Mic60 (MICOS) interacts with Hebp1.

A

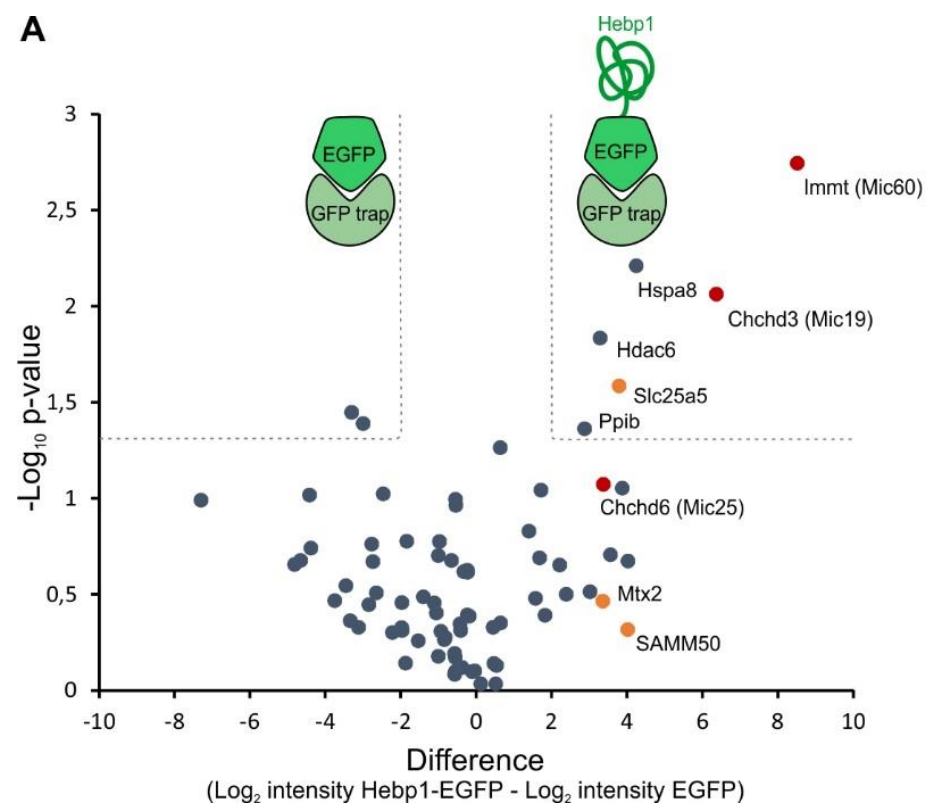

B

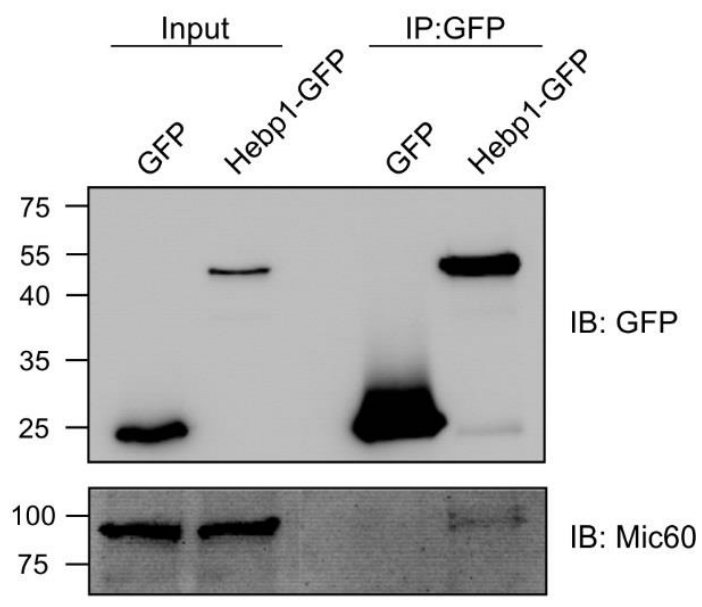

Figure 3-17. Hebp1 interactome reveals its association with mitochondrial contact site and cristae organizing system (MICOS) complex.

(A) Hebp1 interactome obtained by mass spectrometry analysis of proteins co-immunoprecipitated from primary cortical neurons with Hebp1-EGFP or EGFP (negative control). Enrichment of mitochondria contact site complex (MICOS) proteins (red) or MICOS-associated proteins (orange). Dashed line represents a cut-off for significantly different proteins between Hebp1-EGFP and control pulldown with at least 4-fold change. (B) Validation of Hebp1-Mic60 interaction by immunoblotting.

Taken together these data indicate that Hebp1 localizes in the close proximity to mitochondria and interacts with the MICOS complex probably indirectly through associated proteins of outer mitochondria membrane such as SAMM50 or Mtx2. In line with our observation, a very similar pattern of subcellular localization was observed in cells that were transfected with full-length Hebp2/SOUL ${ }^{324}$, hinting towards a potential role of Hebp1 in the regulation of cell death.

\subsubsection{Hebp1 facilitates heme-mediated cytotoxicity}

Heme metabolism, cell death response and AD are tightly interconnected. Dysregulation of proteins linked to heme metabolism has been reported in $A D^{146,147}$. Accumulation of $A \beta$ 
around brain vasculature results in cerebral amyloid angiopathy (CAA), microvessel destruction and leakage of free heme into brain tissue ${ }^{152}$. Due to its strong hydrophobicity, heme is almost exclusively bound to carrier proteins in cellular environment. High concentration of free heme is toxic as has been shown in multiple cell types ${ }^{148,149}$. Therefore, I hypothesized that Hebp1 could serve as a heme buffer to keep the free heme at a low level and protect neurons from the toxic effects of excessive heme. To test this hypothesis, stable clonal HeLa cell lines with the knockout of Hebp1 were generated using CRISPR/Cas9 (Figure $3-18)$.

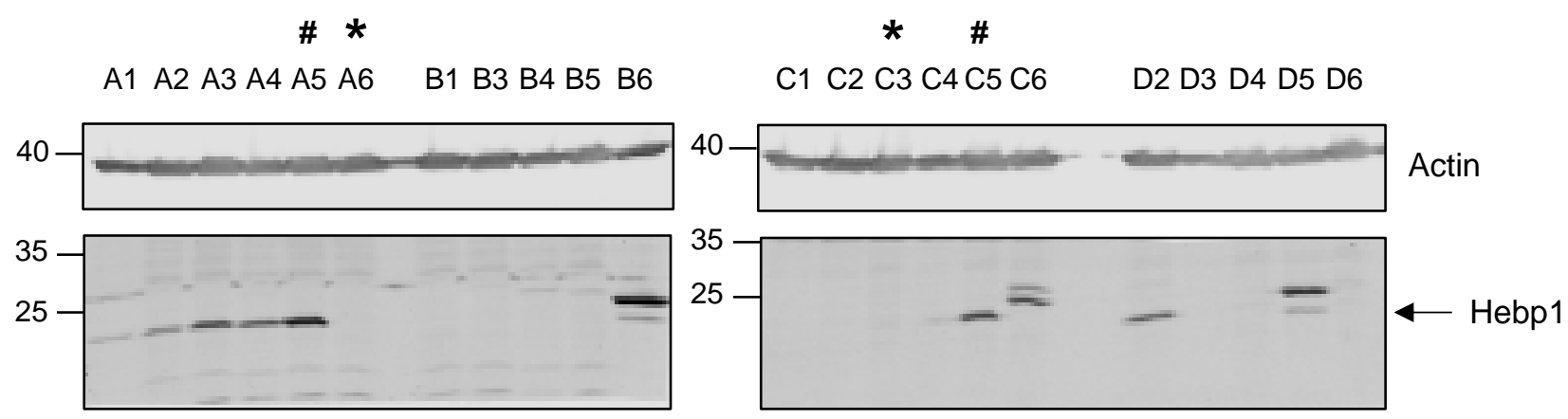

Figure 3-18. Validation of Hebp1 knockout in CRISPR/Cas9-edited clonal HeLa cell lines.

Immunoblotting analysis of Hebp1 expression in independent CRISPR/Cas9-edited clonal HeLa cell lines. Hebp1-KO $\left({ }^{*}\right)$ and wild type (\#) lines were selected and used in further experiments. The following naming of lines is applied: C3 - K01, A6 - K02, C5 - WT1, A5 - WT2.

To analyze the role of Hebp1 in cell response to heme, the generated HeLa lines were treated with exogenous hemin (ferric chloride salt of heme). Cytotoxicity was then assayed by activity of dead cell protease (see Methods). Surprisingly, Hebp1-KO cells demonstrated no response to hemin as compared to vehicle control while cytotoxicity increased by around 50\% for the wild type cells (WT) (Figure 3-19 A). This result indicates that, opposite to the expectations, Hebp1 does not protect cells from excessive heme but rather exacerbates its toxic effect.

To find out whether heme-induced cell death follows the apoptotic pathway and, if so, can Hebp1 depletion protect cells from apoptosis, the activity of caspase 3/7 was assayed in the HeLa lines. Hemin treatment indeed induced apoptosis affecting wild type cells to a higher extent than Hebp1-K0 line (Figure 3-19 D, E). This finding clearly indicates that Hebp1 is essential for activation of apoptosis upon increased concentration of exogenous heme. 
A

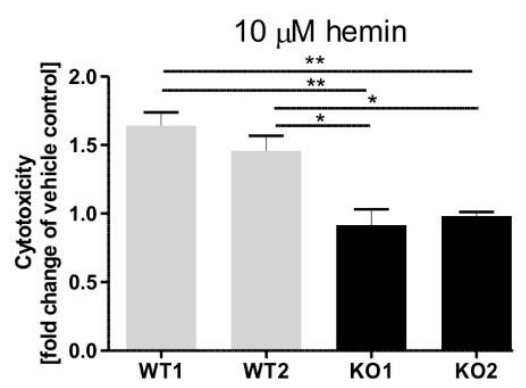

B

$100 \mu \mathrm{M}$ tert-Butyl hydroperoxide

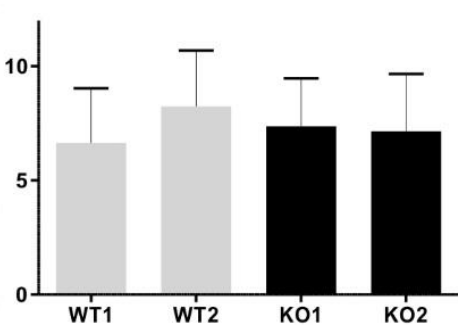

C

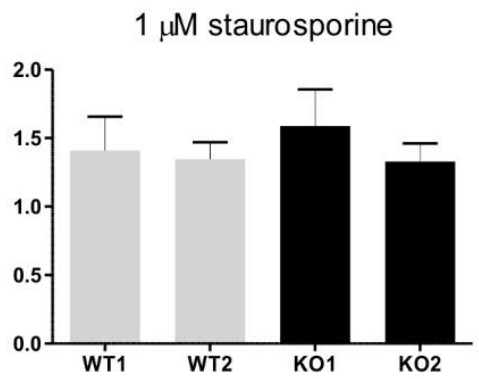

E
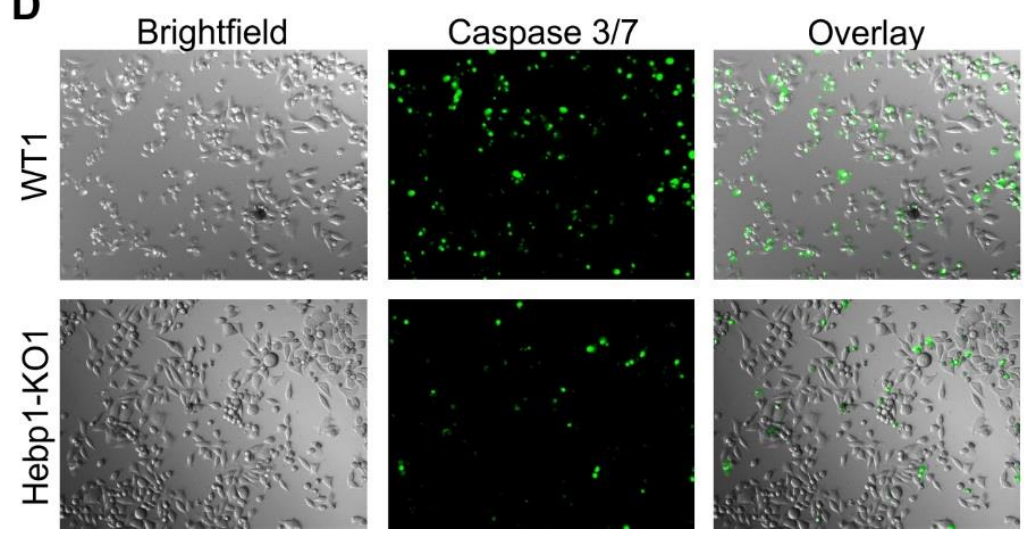

Hemin-induced apoptosis

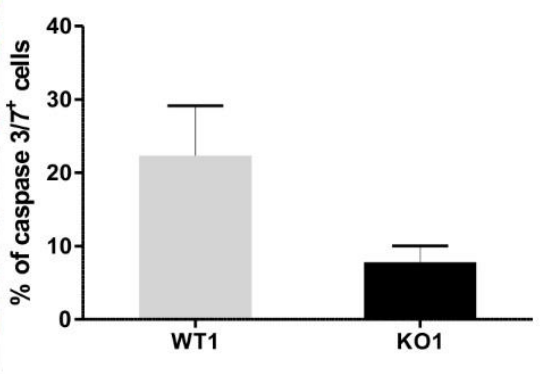

Figure 3-19. Hebp1 is required for apoptotic response upon heme overload.

(A) Measurement of cytotoxicity after 24-hour stimulation with $10 \mu \mathrm{M}$ hemin or vehicle. Hebp1-KO lines are resistant to heme-mediated cytotoxicity. Wild type and Hebp1-KO HeLa cells demonstrate similar response to 3 hour treatment with (B) $100 \mu \mathrm{M}$ tert-Butyl hydroperoxide and (C) $1 \mu \mathrm{M}$ staurosporine.

Hemin-induced cell death follows the apoptotic pathway: (D) Representative images of wild type and Hebp1KO cells treated with $10 \mu \mathrm{M}$ hemin for 24 hours. Apoptotic cells were determined by fluorescent signal of caspase 3/7 activity (see Methods). (E) Hebp1-KO HeLa cells demonstrate increased resistance to apoptosis upon heme overload in comparison to wild type cells.

All bar charts represent mean \pm SEM. Statistical significance in the datasets was assessed by one-way ANOVA followed by Student's t-test comparison for individual pairs of samples: *p value $<0.05$ and ${ }^{* *} p<0.01$,

These results raise the question whether Hebp1 is a general master regulator of apoptosis or its effect on cell death is restricted to heme-mediated toxicity. To this end, Hebp1-KO and wild type cells were subjected to other known inducers of the apoptotic pathway ${ }^{330,331}$. No significant difference in cytotoxicity was observed between $\mathrm{KO}$ and WT lines upon treatment with tert-butyl-hydroperoxide (stable analog of hydrogen peroxide) and staurosporine (Figure 3-19 B and C) which indicates that knockout of Hebp1 can protect cells rather specifically against heme-induced toxicity.

In the next step, I tried to understand through which mechanisms Hebp1 depletion protects cells from hemin-induced apoptosis. I decided to test two processes that accompany 
apoptotic response and can be related to Hebp1 function: the oxidative stress and the maintenance of mitochondrial membrane potential (MMP). First, oxidative stress was investigated in hemin-treated WT and Hebp1-KO cells by measurement of the reduced/oxidized glutathione ratio (GSH/GSSG) (Figure 3-20 A). Hemin has indeed invoked oxidative stress, though no difference in GSH/GSSG ratio between WT and Hebp1-KO cells upon hemin stimulation was detected. Interestingly, a small but significant difference could be observed between WT and KO cells at the basal condition, indicating that Hebp1 may contribute to the maintenance of antioxidative potential under physiological conditions.

A

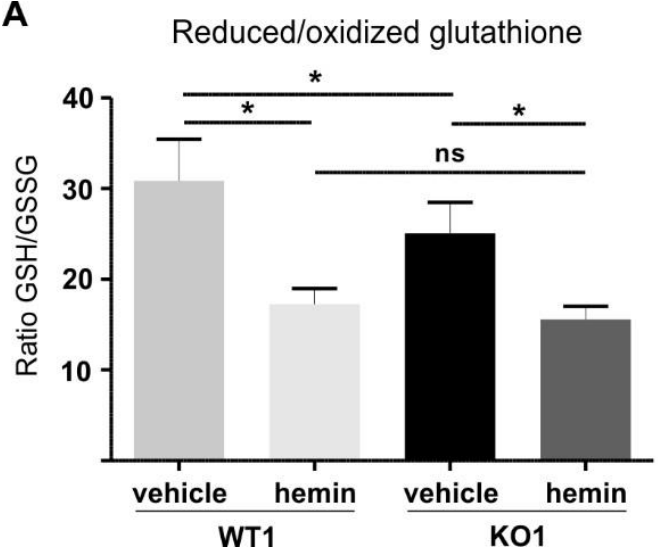

B

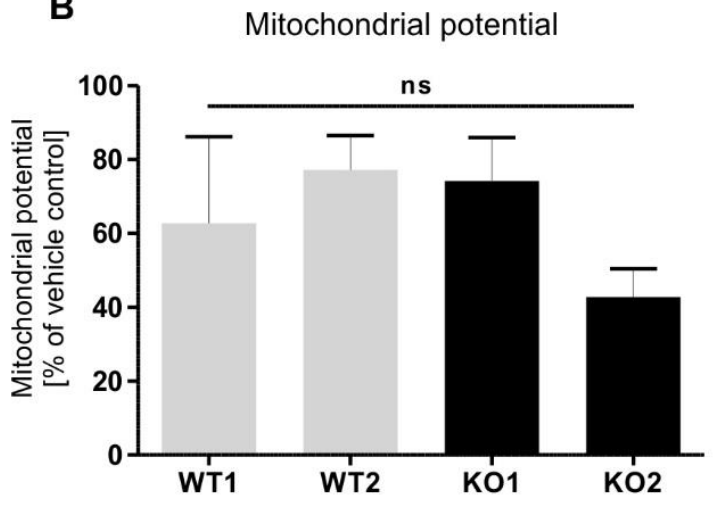

Figure 3-20. Measurement of oxidative stress and mitochondrial membrane potential in hemin-treated wild type and Hebp1-KO HeLa cells.

HeLa cells were treated with $10 \mu \mathrm{M}$ hemin for 24 hours.

(A) Hemin induces oxidative stress as measured by reduced/oxidized glutathione ratio (GSH/GSSG). Hebp1-KO cells exhibit significantly higher levels of oxidative stress compared to wild type at the baseline but not upon hemin stimulation.

(B) Hemin treatment induces reduction of mitochondrial membrane potential in HeLa cells. However, no statistically significant difference was detected between wild type and Hebp1-KO lines.

All bar charts represent mean \pm SEM. Statistical significance in the datasets was assessed by one-way ANOVA followed by Student's t-test comparison for individual pairs of samples: *p value $<0.05$, ns - not significant.

Next, the mitochondrial membrane potential was assayed using a cationic potentiometric dye (see Methods). As expected, hemin treatment indeed led to reduction of the MMP. Nevertheless, no significant effect of Hebp1 knockdown on the MMP could be detected neither upon hemin treatment (Figure 3-20 B), nor at the baseline (data not shown). Taken together, these data suggest that the pro-apoptotic role of Hebp1 upon increased concentrations of free heme is rather independent of the oxidative stress response and regulation of mitochondrial membrane potential or is situated downstream of these processes in the chain of apoptotic events. 
Overall, the experiments utilizing Hebp1-KO cell lines demonstrate that Hebp1 sensitizes cells to apoptosis in response to heme overload. Such an effect of Hebp1 in not manifested through the altered response of cells to oxidative stress or differences in mitochondrial membrane potential. The exact mechanism of such a pro-apoptotic effect of Hebp1 requires further investigations. Nevertheless, the involvement of Hebp1 in the cell death response is extremely interesting in the context of Alzheimer's disease and its progression. The implications of these findings will be discussed in the next chapter of this thesis. 


\section{Discussion}

In this thesis, the changes in brain proteome and phosphoproteome were investigated along the progression of Alzheimer's disease in $3 \times \mathrm{Tg}-\mathrm{AD}$ mice. These data provided valuable information on alterations in protein expression levels at specific stages of $\mathrm{AD}$ and allowed to determine the affected biological processes and pathways. A special aspect of this project was the focus on identifying proteins whose expression differed significantly between $3 \times \mathrm{Tg}$ $\mathrm{AD}$ and control mice at the presymptomatic stage. The presymptomatic markers identified in this study included both proteins previously described to be involved in AD as well as new candidates. A closer examination of one such novel protein, Hebp1, revealed its increased expression in the brains of patients afflicted with rapidly-progressing forms of AD. Further functional analyses demonstrated neuronal expression of Hebp1 in the brain, its association with mitochondria via MICOS complex and the role in apoptotic response to cytotoxic levels of exogenous heme. In this chapter, the significance of these results will be discussed in a broader context of $\mathrm{AD}$ pathogenesis. Strengths and limitations of applied experimental approaches will be further elaborated here.

\subsection{Analysis of biological processes affected in the brain of $3 \times \mathrm{Tg}-\mathrm{AD}$ mice}

Bioinformatic analyses of proteomic and phosphoproteomic data identified several groups of proteins with altered expression in $3 \times \mathrm{Tg}-\mathrm{AD}$ mice at specific stages of the disorder (Figures 3-4, 3-5, 3-6 and 3-7). Here, I discuss in detail the affected biological processes that are most relevant to $\mathrm{AD}$.

\subsubsection{Regulation of microtubule stability}

Cumulative alterations in expression of proteins involved in microtubule dynamics were among the most prominent differences observed between the brain proteomes of the control and $3 \times \mathrm{Tg}-\mathrm{AD}$ mice (Figure 3-6). The phosphorylation of cytoskeletal proteins was also significantly upregulated in the transgenic mice. Interestingly, while the expression changes of cytoskeleton-related proteins became prominent only by the late stage (18 months), their phosphorylation was already significantly affected at the presymptomatic time point (Figure 3-8). 
Microtubule instability in AD is primarily associated with tau hyperphosphorylation ${ }^{19}$. Indeed, I identified several phosphosites of tau to be phosphorylated at higher levels in the brain of $3 \times \mathrm{Tg}-\mathrm{AD}$ mice. Interestingly, hyperphosphorylated tau can sequester normal tau as well as other MAPs ${ }^{35,332}$ leading to formation of NFTs. Remarkably, in this study, I also found multiple phosphosites of MAP1, MAP2, MAP4 and MAP9 to be differentially phosphorylated between the control and $3 \times \mathrm{Tg}-\mathrm{AD}$ mice. A comprehensive analysis of human brain phosphoproteome also identified a range of upregulated phosphosites in these MAPs in AD stressing their importance for the disease in humans ${ }^{275}$. In general, the phosphorylation of MAPs impacts microtubule dynamics, though the precise function of individual phosphosites are not well understood yet ${ }^{333-335}$. Further assessment of site-specific MAP phosphorylation is thus required to obtain a comprehensive overview of microtubule regulation in AD.

Increased phosphorylation of tau and other MAPs could be detected at the presymptomatic time point already. This is particularly interesting given the findings of previous studies demonstrating that human neurons can live for decades with hyperphosphorylated tau before dying 336,337. My results also indicate that the elevated phosphorylation of cytoskeletal proteins can be seen early in the disease, long before the development of NFTs at a later age and may drive pathological changes during its silent stage.

As aforementioned, many AD-related phosphosites of tau were indeed upregulated in the dataset (Table 4-1). On the other hand, phosphorylation of several "classical" tau sites was not significantly affected in $3 \times \mathrm{Tg}-\mathrm{AD}$ mice even at the time point 18 months (Table 4-1). There are few potential explanations to such outcome. First, in this study, a soluble fraction of brain proteins was used for the phosphoproteomics, while hyperphosphorylated tau can be found predominantly in the insoluble fraction ${ }^{338}$. Second, special conditions may be required for hyperphosphorylation of tau that are not fully fulfilled in the mouse brain 339,340 . Third, mutated human tau (P301L) overexpressed in 3×Tg-AD mice has lower affinity to microtubules but is not necessarily more prone to phosphorylation 234,341 . Additionally, previous studies demonstrated weaker hyperphosphorylation of some classical tau phosphosites in $3 \times$ Tg-AD mice ${ }^{342,343}$ in comparison to humans ${ }^{275}$. My results support these observations and suggest that the data obtained in $3 \times \mathrm{Tg}-\mathrm{AD}$ mice should be extrapolated cautiously on AD in humans. 
Table 4-1. Phosphorylation profile of tau at time point 18 months.

Phosphorylation of individual tau phosphosites detected in phosphoproteomics analysis at time point 18 months and comparison with the previously published data. Legend: Aa - amino acid residue, $\mathrm{m}$ - multiplicity (the number of phosphorylated amino acid residues on the identified peptide). Last column (D) indicates whether corresponding phosphosite was identified to be significantly upregulated in human AD tissue according to the study of Dammer and collegues ${ }^{275}$. Significantly regulated phosphosites in this study are marked in red.

\begin{tabular}{|c|c|c|c|c|c|c|c|c|}
\hline \multirow[t]{2}{*}{ Aa } & \multicolumn{2}{|c|}{$\begin{array}{l}\text { Amino acid } \\
\text { position }\end{array}$} & \multirow[t]{2}{*}{ Sequence window } & \multirow[t]{2}{*}{$\mathbf{M}$} & \multirow{2}{*}{$\begin{array}{c}\text { p- } \\
\text { value } \\
\text { (t-test) }\end{array}$} & \multirow{2}{*}{$\begin{array}{c}\log _{2} \\
\text { ratio } \\
\text { AD/Ctrl }\end{array}$} & \multirow{2}{*}{$\begin{array}{c}\text { Known in } \\
\text { AD }\end{array}$} & \multirow[t]{2}{*}{ D } \\
\hline & Mouse & Human & & & & & & \\
\hline$S$ & 167 & - & RIPAKTTPSPKTPPGSG & 2 & 0,742 & 0,153 & & \\
\hline $\mathrm{T}$ & 170 & 181 & AKTTPSPKTPPGSGEPP & 1 & 0,843 & $-0,165$ & & - \\
\hline $\mathrm{T}$ & 170 & 181 & AKTTPSPKTPPGSGEPP & 2 & 0,827 & 0,143 & & - \\
\hline S & 187 & 198 & KSGERSGYSSPGSPGTP & 2 & 0,031 & $-0,441$ & & - \\
\hline$S$ & 188 & 199 & SGERSGYSSPGSPGTPG & 2 & 0,622 & $-0,017$ & Onishi et al., & - \\
\hline S & 188 & 199 & SGERSGYSSPGSPGTPG & 1 & 0,098 & 0,317 & $\begin{array}{l}2014^{344}, \\
\text { Henkins et }\end{array}$ & - \\
\hline $\mathrm{S}$ & 191 & 202 & RSGYSSPGSPGTPGSRS & 2 & 0,319 & 1,271 & al., $2012^{345}$ & - \\
\hline$S$ & 199 & 210 & SPGTPGSRSRTPSLPTP & 2 & 0,636 & 0,032 & & + \\
\hline S & 199 & 210 & SPGTPGSRSRTPSLPTP & 3 & 0,092 & 0,795 & & - \\
\hline $\mathrm{T}$ & 201 & 212 & GTPGSRSRTPSLPTPPT & 3 & 0,083 & 0,441 & & + \\
\hline$S$ & 203 & 214 & PGSRSRTPSLPTPPTRE & 1 & 0,958 & 0,153 & & + \\
\hline S & 203 & 214 & PGSRSRTPSLPTPPTRE & 2 & 0,563 & 0,051 & & + \\
\hline S & 203 & 214 & PGSRSRTPSLPTPPTRE & 3 & 0,083 & 0,441 & & - \\
\hline $\mathrm{T}$ & 206 & 217 & RSRTPSLPTPPTREPKK & 2 & 0,563 & 0,051 & & + \\
\hline $\mathrm{T}$ & 206 & 217 & RSRTPSLPTPPTREPKK & 3 & 0,128 & 0,491 & & - \\
\hline $\mathrm{T}$ & 220 & 231 & PKKVAVVRTPPKSPSAS & 2 & 0,101 & 0,467 & $\begin{array}{l}\text { Lasagna- } \\
\text { Reeves, } \\
2014^{346}, \\
\text { Jicha et al., }\end{array}$ & - \\
\hline$S$ & 224 & 235 & AVVRTPPKSPSASKSRL & 2 & 0,101 & 0,467 & 1997347 & \\
\hline $\mathrm{S}$ & 251 & 262 & NVKSKIGSTENLKHQ & - & - & - & $\begin{array}{l}\text { Gu et al., } \\
2016^{348}\end{array}$ & + \\
\hline $\mathrm{Y}$ & 383 & 394 & TDHGAEIVYKSPVVSGD & 3 & 0,146 & 0,995 & & - \\
\hline$S$ & 385 & 396 & HGAEIVYKSPVVSGDTS & 2 & 0,931 & $-0,017$ & & + \\
\hline S & 385 & 396 & HGAEIVYKSPVVSGDTS & 3 & 0,478 & 0,000 & , & + \\
\hline S & 389 & 400 & IVYKSPVVSGDTSPRHL & 3 & 0,459 & $-0,006$ & & + \\
\hline$S$ & 389 & 400 & IVYKSPVVSGDTSPRHL & 2 & 0,038 & 0,206 & & + \\
\hline $\mathrm{T}$ & 392 & 403 & KSPVVSGDTSPRHLSNV & 1 & 0,912 & $-0,109$ & Noble et a & + \\
\hline $\mathrm{T}$ & 392 & 403 & KSPVVSGDTSPRHLSNV & 3 & 0,354 & $-0,019$ & & + \\
\hline S & 393 & 404 & SPVVSGDTSPRHLSNVS & 3 & 0,404 & $-0,270$ & & + \\
\hline S & 393 & 404 & SPVVSGDTSPRHLSNVS & 1 & 0,328 & 0,360 & & - \\
\hline $\mathrm{S}$ & 393 & 404 & SPVVSGDTSPRHLSNVS & 2 & 0,042 & 0,338 & & - \\
\hline$S$ & 401 & 412 & SPRHLSNVSSTGSIDMV & 3 & 0,354 & 0,513 & & - \\
\hline S & 402 & 413 & PRHLSNVSSTGSIDMVD & 3 & 0,387 & 0,784 & & - \\
\hline S & 402 & 413 & PRHLSNVSSTGSIDMVD & 2 & 0,151 & 0,210 & & - \\
\hline
\end{tabular}




\subsection{2 mRNA processing}

Several proteins in our dataset exhibited gradual increases in expression that correlated with the disease progression in $3 \times \mathrm{Tg}-\mathrm{AD}$ mice. These included proteins that are involved in mRNA processing such as Matrin-3 and Nono that form a complex involved in DNA damage response and recognition and retention of incorrectly processed mRNA in the nucleus ${ }^{352,353}$. Similar changes in expression pattern were detected for Hnrnpm and Hnrnpl, which regulate alternative splicing 354,355 . Bai and colleagues have previously found U1 snRNP components to be enriched in insoluble brain proteome of $\mathrm{AD}$ patients and demonstrated impaired splicing of AD-related transcripts 266 .

Increased phosphorylation of SRRM1-2 proteins that are directly involved in the regulation of splicing was detected at 18 months in $3 \times \mathrm{Tg}-\mathrm{AD}$ mice. Nonetheless, the role of SRRM phosphorylation in the regulation of this process is not known. Strikingly, a recent phosphoproteomics study of brain samples from four different mouse models of AD $(5 \times F A D$, Tg2576, PS1-dE9, PS2N141I) revealed an increased phosphorylation of several SRRM2 phosphosites 277 . Interestingly, the same report demonstrated an upregulation of SRRM2 phosphorylation in the mouse model of tauopathy. Furthermore, the levels and the splicing of SRRM2 itself are dysregulated in Parkinson's disease. Taken together, these results hint towards a broader importance of impaired RNA processing in neurodegenerative disorders 356 .

The kinase prediction analysis revealed that most of the upregulated phosphosites on SRRM proteins were potential substrates of CLK kinases (Figure 3-10). Increased CLK activity in the brains of $\mathrm{AD}$ patients was reported previously ${ }^{357}$ and was proposed to be associated with more frequent exon 10 skipping during the splicing of tau ${ }^{358}$. It was also suggested that the effects of the elevated CLK activity in AD are manifested through phosphorylation of SRproteins including SRRM1-2358,359. However, no experimental evidence was provided to support this hypothesis. Importantly, my analysis demonstrates for the first time that the hyperphosphorylation of SRRM1-2 proteins in AD pathology is attributed to CLK kinases. Kinases are prospective therapeutic targets since their activity can be inhibited pharmacologically. A range of small molecule inhibitors of CLKs was developed over the last decade ${ }^{359}$. CLKs have relatively few known substrates apart from SR-proteins which is 
advantageous for minimization of potential off-target effects. CLKs are thus promising drug targets for $\mathrm{AD}$ especially given the concomitant increase of their activity with the aggravation of pathology in $3 \times \mathrm{Tg}$-AD model.

This study supports the previous findings indicating a disturbance in mRNA processing during $A D^{266,360,361}$. Despite being a promising direction of research to follow, implications of mRNA processing in AD have not been studied extensively. Therefore, thorough analyses of RNA splicing using contemporary RNA-seq techniques have to be performed to determine how exactly splicing events are altered in $\mathrm{AD}$ and what are the transcripts that are affected the most by this disorder.

\subsubsection{Inhibition of serine proteases}

Several inhibitors of serine proteases (serpins) demonstrated a coordinated declining expression over the course of $\mathrm{AD}$ progression in $3 \times \mathrm{Tg}-\mathrm{AD}$. Interestingly, a recent study of the hippocampal proteome in $5 \times \mathrm{FAD}$ mouse model of $\mathrm{AD}$ also identified downregulation of serpins $^{362}$. In contrast, accumulation of alpha 1-antitrypsin (serpina1c) in $\mathrm{A} \beta$ plaques of $\mathrm{AD}$ patients was detected previously ${ }^{363}$. Such discrepancy between the data can be explained by the recruitment of serpins to the $A \beta$ plaques and a subsequent depletion of their levels in the soluble brain fraction. Several serine proteases can cleave APP ${ }^{364}$. Therefore, the recruitment of serpins to the $A \beta$ plaques could be a response mechanism aimed to reduce deleterious APP processing.

Regulation of protease activity is critical in AD not only from the standpoint of proteolytic APP cleavage but also because of many other functions. Protease inhibitors regulate composition of extracellular matrix, synaptic plasticity, immune response and other processes directly related to $\mathrm{AD}$ pathogenesis 365,366 . For instance, the inhibition of serine proteases by serpins has been proposed as a mechanism of protection from the excessive synapse loss ${ }^{364}$. Serpins also play an important role in apoptosis 367.

Among the three serine protease inhibitors identified to be gradually downregulated in $3 \times$ Tg-AD mice with age, alpha1-antitrypsin has been studied most extensively as mentioned in references above. Less is known about serpin A3K (Serpina3k) and murinoglobulin-1 (Mug1). Serpina3k has been previously shown to inhibit inflammatory response in corneal endothelium by interfering with TNF- $\alpha$ signaling ${ }^{368,369}$. Additionally, Serpina3k protects 
retinal Müller cells from oxidative stress and cell death, though the exact mechanisms of such protection remain unknown ${ }^{370}$. Even less information is available on Mug-1 and its function. Mug1 is expressed predominantly in CA1-3 hippocampal regions where it regulates synaptic plasticity by inhibiting neuropsin ${ }^{371}$. Interestingly, Mug1 exhibits structural similarity to A2M. The proteomic analysis performed in this study did not reveal any significant changes of A2M expression at the time points 2-12 months. However, this protein was significantly downregulated at the preterminal stage of the disorder (18 months) following the same direction of change as serpina1a, serpina3k and Mug1 (Supplementary table 1). Importantly, the deletion of 5'-splice site of exon II in $A 2 M$ gene confers an increased risk for developing $\mathrm{AD}^{372}$. Additionally, elevated levels of A2M protein were identified in CSF of patients with $\mathrm{MCI}$ and fully-developed $\mathrm{AD}^{373}$. A2M inhibits maturation of nerve growth factor (NGF) in vitro and can induce neuronal death through interaction with surface receptor p75374. Nevertheless, the exact role of $\mathrm{A} 2 \mathrm{M}$ protein in $\mathrm{AD}$ pathogenesis is not known. Collectively, the available information on the identified serine protease inhibitors point out their role in inflammation and cell death response which is in line with the other findings in this thesis.

Serpins hold a potential as therapeutic targets, since their levels in the brain can be restored through injection of recombinant protein. This approach was recently tested with metalloprotease inhibitor TIMP2 which was injected intraperitoneally to reach the brain and improve the cognitive function of aged mice ${ }^{375}$. It would be thus interesting to test whether a manipulation of systemic serpin levels can ameliorate pathology and cognitive decline in AD mice.

\subsubsection{Cell death}

Neuronal death is the key process responsible for the cognitive decline in AD patients ${ }^{376}$. The analysis of proteomic data with IPA revealed an activation of processes related to cell death in 6-month-old $3 \times \mathrm{Tg}$ - $\mathrm{AD}$ mice. Whether the loss of neurons is happening in $3 \times \mathrm{Tg}-\mathrm{AD}$ mice remains an open question ${ }^{377}$. Given the data from AD patients and other mouse models of the disorder, it is unlikely that neuronal loss would happen early on in the disease progression before the initial accumulation of $A \beta^{378,379}$. On the other hand, overexpression of proteins counteracting apoptotic response has been previously shown to decrease pathology in $3 \times \mathrm{Tg}$ $\mathrm{AD}$ mice ${ }^{110}$. The same study demonstrated activation of caspases 9 and 3 in the CA regions of 
hippocampus in young transgenic mice, which is in agreement with the data obtained in this thesis. It is possible that the proapoptotic proteins such as caspases exacerbate AD pathology through other mechanisms that are not strictly related to cell death ${ }^{380,381}$. Alternatively, early changes in expression of genes related to apoptosis may not lead to an immediate cell death but rather predispose neurons to it. This is particularly interesting in light of my findings regarding early upregulation of Hebp 1 expression in $3 \times \mathrm{Tg}-\mathrm{AD}$ mice and its newly found role in apoptotic response.

\subsection{Heme-binding protein 1 as a novel candidate involved in early stages of AD pathogenesis}

Hebp1 has not been studied extensively. The available publications on this protein are restricted to its heme-binding properties and a role in immune response (discussed later). No prior report associates Hebp1 with the brain function. It is therefore especially striking that Hebp1 was identified here as one of the proteins with the highest upregulated expression in 3×Tg-AD mice. Therefore, understanding the functions of Hebp1 in the brain is a critical step for deciphering its role in AD pathology.

\subsubsection{Association of Hebp1 with MICOS complex}

Immunohistological assessment of mouse brain revealed that Hebp1 is predominantly a neuronal protein (Figure 3-14 C). The increase of Hebp1 expression could be detected in all major brain areas of $3 \times \mathrm{Tg}-\mathrm{AD}$ mice. Interestingly, the highest levels of Hebp1 were observed in hippocampus, the brain region that is most affected by $\mathrm{AD}^{382}$.

To better understand the function of Hebp1 in neurons, I performed an IP-MS analysis of Hebp1 binding partners (Figure 3-17). This analysis revealed that Hebp1 interacts with the members of MICOS complex and associated proteins. MICOS is a multiprotein complex residing in the inner mitochondria membrane and is responsible for the formation and maintenance of mitochondrial cristae junctions ${ }^{383}$. According to the Co-IP/MS data, the core component of MICOS complex, Mic60, was the most enriched protein in the Hebp1-EGFP pulldown fraction (Supplementary table 3). Other constituents of the complex, Mic19 and Mic25, also co-immunoprecipitated with Hebp1-EGFP. The known interactors of MICOS such 
as SAMM50, metaxin 2 and ADP/ATP translocase 2 (Slc25a5) also bound Hebp1-EGFP in neurons 384,385 . SAMM50 together with metaxin 1 and 2 form sorting and assembly machinery complex (SAM) in the outer mitochondrial membrane ${ }^{386}$. SAMM50 directly interacts with Mic19387 and connects SAM and MICOS complexes to form mitochondrial intermembrane space bridging supercomplex (MIB) that spans both mitochondrial membranes ${ }^{386,388}$.

SAM complex is involved in the recognition and insertion of $\beta$-barrel proteins into the outer mitochondrial membrane ${ }^{389}$. Interestingly, Hebp1 forms a distorted beta-barrel structure ${ }^{322}$. Hebp1 also does not possess canonical mitochondria-targeting sequence ${ }^{390}$. Taking into account an overlap of Hebp1-EGFP localization with the surface of mitochondria (Figure 316), it can be speculated that a portion of Hebp1 could be inserted into the outer mitochondrial membrane by SAM complex. However, an elegant study utilizing the peroxidase-mediated proximity biotinylation demonstrated that Hebp1 is not an integral part of outer mitochondrial membrane but rather localizes in close proximity at its cytosolic side $^{327}$. These data also agree with my observations of Hebp1 localization in neurons.

\subsubsection{Hebp1 and heme transport}

Before discussing the role of Hebp1 in apoptotic response, one more possible function of Hebp1 should be considered. It is known from the previous studies that Hebp1 can efficiently bind heme and other protoporphyrinogens ${ }^{320-322}$. Importantly, heme biosynthesis and metabolism depends on the efficient transport of heme and its precursors between mitochondria and cytosol ${ }^{391}$. Heme is a highly hydrophobic molecule and thus requires protein carriers to mediate its trafficking in and out of mitochondria ${ }^{392}$. Surprisingly, it is still not known which proteins are responsible for the export of mature heme from mitochondria to cytosol or import of heme precursors such as coproporphyrinogen III inside the mitochondria ${ }^{392}$ (Figure 4-1). The data obtained in this project indicate that Hebp1 resides close to the outer mitochondrial membrane and can potentially serve as an acceptor of heme exported from mitochondria (Figure 4-1). Heme can then be redistributed by Hebp1 to other cytosolic proteins that require heme as their co-factor (e.g. globins or nitric oxide synthase ${ }^{393}$ ). Alternatively, Hebp1 can facilitate the transport of coproporphyrinogen III into mitochondria or serve as a heme buffer, keeping the levels of cytosolic heme under control (Figure 4-1). Nevertheless, the involvement of Hebp1 in the heme transport was not explored 
in this thesis and the proposed ideas are just hypotheses that require further validation. Experiments tracing radiolabeled heme in Hebp1-depleted cells (Hebp1-KO HeLa lines generated in this thesis project) could help to explore the role of Hebp1 in heme trafficking.

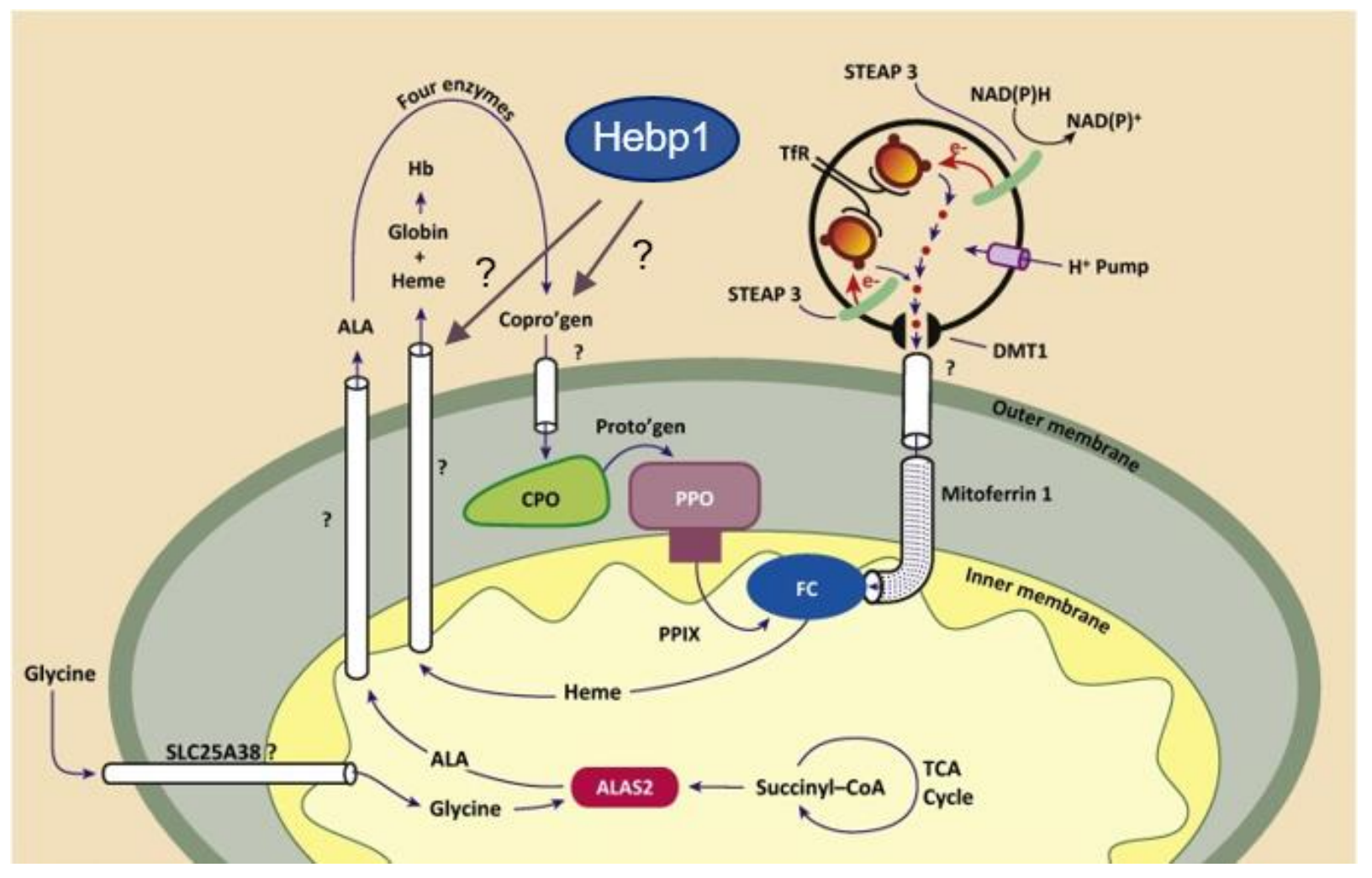

Figure 4-1. Hypothetical role of Hebp1 in heme metabolism.

See explanation in the main text. Abbreviations: ferrochelatase (FC), protoporphyrin IX (PPIX), coproporphyrinogen oxidase (CPO), hemoglobin ( $\mathrm{Hb}$ ), protoporphyrinogen oxidase (PPO), transferrin receptor (TfR).

Adapted and modified from Ponka et al., $2017^{392}$.

\subsubsection{Hebp1 and cell death}

Mitochondria are the centerpiece in the cascade of events during apoptosis. The relocation of proteins from cytosol to outer mitochondrial membrane upon cell stress or mitochondria damage can be directly implicated in cell death and neurodegeneration as demonstrated by the example of parkin in Parkinson's disease ${ }^{86}$. Interestingly, heme-binding protein 2 (SOUL), a homolog of Hebp1, promotes cell death presumably through recruitment to mitochondria and permeabilization of its outer membrane ${ }^{324,325}$. Moreover, the newly identified binding partner of Hebp1, Mic60, is also an important player in cell death. Loss of Mic60 increases the rate of apoptosis due to the dissipation of cristae junctions and intensifies leakage of 
cytochrome c from mitochondria to cytosol ${ }^{394}$. Together, these findings suggest that Hebp1 itself can be involved in the regulation of cell death response.

Indeed, I observed that Hebp1-depleted HeLa cells become resistant to hemin-mediated toxicity. The cytoprotective effect of Hebp1 knockout is specific to heme as the rate of cell death did not differ significantly between Hebp1-KO and wild type cells upon treatment with other cytotoxic compounds (Figure 3-19). But what could be the implications of hememediated cell death for AD? Degradation of heme by heme oxygenase-1 (HMOX1) was demonstrated to reduce cytotoxicity caused by A $\beta_{1-42}$ peptide in SH-SY5Y cells ${ }^{395}$. HMOX1 was not identified in the proteomic analysis in this study. However, binding partner of HMOX1, biliverdin reductase B (Blvrb), together with Hebp1 were one of the most upregulated proteins at the presymptomatic time point (Figure 3-11). Importantly, Blvrb catalyzes the second and the final step of heme degradation reducing biliverdin to bilirubin ${ }^{396}$. Increased levels of HMOX1 and biliverdin reductase isoform A (Blvrba) in the brains of AD patients were reported previously ${ }^{146,397,398}$. It is possible that the levels of Blvrb increase in response to the increased concentration of free heme in the brain of $3 \times \mathrm{Tg}-\mathrm{AD}$ mice to enhance its degradation.

Whether the levels of free heme in $\mathrm{AD}$ brain are significantly elevated remains unclear. Nevertheless, several lines of evidence point towards this possibility. Heme synthesis is impaired in $\mathrm{AD}$ and accumulation of immature heme species can be a potential source of heme overload ${ }^{147}$. Excessive heme in the brain can also originate from bloodstream. Cerebral amyloid angiopathy contributes significantly to AD both in human and the $3 \times \mathrm{Tg}$-AD model starting from the early stage of the disease ${ }^{399,400}$. CAA may lead to the disruption of brain vasculature and a consequent release of heme outside the vessels 152,401 . CAA is also associated with apoptosis ${ }^{402,403}$ and thus creates a possible link between upregulation of Hebp1 early in $\mathrm{AD}$ and its newly found role in mediation of heme cytotoxicity.

To understand through which mechanisms Hebp1 depletion protects cells from heme overload, I have examined the changes in oxidative stress and mitochondrial membrane potential in Hebp1-KO and wild type cells upon hemin treatment. Both parameters were affected by addition of hemin to cell culture. However, Hebp1 knockout influenced neither of them significantly (Figure 3-20). These observations lead to two possible conclusions. First, 
the protective effect of Hebp1 depletion is not manifested through the regulation of oxidative stress or mitochondrial membrane potential. Second, Hebp1 affects apoptosis downstream of these processes. The mechanism by which Hebp1 depletion protects cells from apoptotic response to hemin treatment remains unclear and therefore requires further investigations.

It also has to be mentioned that no striking abnormalities of mitochondria morphology were detected in Hebp1-KO HeLa cells or Hebp1-overexpressing neurons (data not shown). A closer look at the mitochondria structure (for example, by means of electron microscopy) can determine whether cristae junctions are impaired upon Hebp1 knockout or overexpression. Such experiments can verify if a link between Hebp1, MICOS complex and apoptosis does exist.

\subsubsection{Proteolytic cleavage of Hebp1 in immune response}

Hebp1 also poses interest for its potential signaling function. Two studies demonstrated that $\mathrm{N}$-terminal cleavage of Hebp1 by cathepsin D results in generation of 21 amino acid long peptide called F2L that is capable of binding FPRL1/FPR2 receptor on the surface of mouse neutrophils and promote their migration ${ }^{404,405}$. In the mouse brain, FPR2 is expressed predominantly by activated microglia ${ }^{406}$. Moreover, FPRL1-positive microglia was shown to be recruited to $\mathrm{A} \beta$ plaques in $\mathrm{AD}$ patients ${ }^{407}$. In our dataset, expression of the Hebp 1 protease cathepsin D also strongly correlates with aging which indicates the possibility of the progressive F2L accumulation in $3 \times \mathrm{Tg}$ - $\mathrm{AD}$ mice with age. Increased expression of cathepsin $\mathrm{D}$ in hippocampus of $\mathrm{AD}$ patients was also reported previously ${ }^{408}$. Thus, it is possible that cleavage of Hebp1 by cathepsin D in neurons may additionally generate the soluble F2L peptide to recruit activated microglia and modulate inflammatory response during AD. Such

hypothesis can be tested by intracerebral infusion of F2L peptide to wild type and 3×Tg-AD mice and subsequent monitoring of microglia activation by imaging techniques.

\subsection{Advantages and caveats of applied experimental procedures}

\subsubsection{Mouse model}

To address the aims of this thesis, the use of an animal model was inevitable. Mouse models have contributed greatly to studies of AD mechanisms and preclinical drug development. The 
$3 \times$ Tg-AD mouse model used in this study develops major neuropathological signs associated with AD. Over the last fifteen years, these mice became the workhorse of AD research and were used in over 1000 published studies, making them one of the most popular animal models in AD research 55 .

Despite the evident advantages, $3 \times \mathrm{Tg}-\mathrm{AD}$ mice also exhibit several significant limitations. Like most other AD models, $3 \times \mathrm{Tg}$-AD mice overexpress mutated human transgenes (APP and tau) under the control of an exogenous promoter. Such overexpression has been linked to several potential artifacts ${ }^{224}$. For example, it was suggested that the pathological changes in mice overexpressing APP are not attributed exclusively to the increased production of A $\beta$ but rather elevated expression of APP itself ${ }^{409,410}$. A recent study utilizing newly-generated APP knock-in mice confirmed some of these suspicions ${ }^{411}$. Extreme overexpression of APP may interfere with kinesin-mediated axonal trafficking ${ }^{412}$, activate Cdk5 through p35 cleavage by calpain protease $\mathrm{e}^{204}$ and induce synapse loss and cell death through increased production of C-terminal APP fragments ${ }^{413}$.

At the same time, it should be mentioned that $3 \times \mathrm{Tg}-\mathrm{AD}$ mice overexpress APP and tau at relatively mild levels (cumulative expression of endogenous and ectopic APP and tau proteins in 3×Tg-AD model was increased up to about 1.5-fold level of control in this study). Such a mild expression of introduced transgenes in $3 \times \mathrm{Tg}-\mathrm{AD}$ model may slightly delay or reduce AD-related neuropathological changes. Although $3 \times \mathrm{Tg}-\mathrm{AD}$ mice used in this study exhibited $A \beta$ plaques (Figure 3-1), their density was somewhat lower in comparison to original reports from Oddo and colleagues ${ }^{233,235}$, possibly due to a genetic drift in the colony ${ }^{414}$.

Few considerations should be made with regard to tau-P301L transgene. The phosphoproteomic analysis revealed the upregulation of several tau phosphosites already at the presymptomatic stage. At the same time several phosphosites of tau known for their involvement in $\mathrm{AD}$ remained unaffected in $3 \times \mathrm{Tg}-\mathrm{AD}$ model (see chapter "Regulation of microtubule stability"). As discussed in that section, the magnitude of observed tau hyperphosphorylation in $3 \times \mathrm{Tg}-\mathrm{AD}$ mice was low in comparison to terminal AD patients. My observations point out that the differences exist in the behavior of tau protein in mouse and human brain affected by AD. 
Taking into account the potential artifacts related to the nature of genetic mouse models, validation of the key findings using the brain samples obtained from $A D$ patients was particularly important. The two most prominent presymptomatic markers identified in this study, Hebp1 and Glo1, were also expressed at significantly higher levels in the brains of rpAD patients confirming the relevance of the data obtained in $3 \times \mathrm{Tg}-\mathrm{AD}$ model for the disease in humans.

\subsubsection{Sample processing and mass spectrometry}

$\mathrm{AD}$ affects primarily hippocampus and various cortical regions ${ }^{415,416}$. To focus on the most pronounced point of AD pathology, previous proteomic studies often utilized proteins isolated specifically from these regions ${ }^{262}$. Nevertheless, recent studies have demonstrated that abnormalities in cerebellum and brain stem can be detected in AD as well ${ }^{417,418}$. To capture an entire spectrum of AD proteome across all brain regions I used whole brain as an input sample. Such approach indeed allowed for uncovering the differences in brain proteome that are not exclusively related to hippocampus or cortex. For instance, immunoblotting analysis revealed increased levels of Hebp 1 in all major regions of the brain in $3 \times \mathrm{Tg}-\mathrm{AD}$ mice, including cerebellum and brain stem (Figure 3-14 A). At the same time, I acknowledge that the use of whole brain sample could potentially minimize the observed differences in expression levels restricted to particular regions of the brain.

Shotgun mass spectrometry is a reliable technique for relative quantification of protein levels between different biological samples. It is a powerful method for explorational studies aiming at identification of unknown proteins involved in a certain biological process or disease.

Shotgun mass spectrometry operates in the data-dependent acquisition mode (DDA). In DDA, the most abundant peptides are selected automatically for fragmentation and acquisition of MS2 spectra which results in identification of the individual peptides ${ }^{419}$. Therefore, DDA is intrinsically biased towards identification of highly-abundant peptide species. This poses a difficulty for identification of low abundant proteins in complex biological samples.

To minimize this problem, subcellular fractionation of the brain was performed and a soluble fraction of proteins was used for proteomic and phosphoproteomic analysis in this study. While this strategy reduced identification of membrane proteins potentially relevant to $A D$ 
(e.g. synaptic proteins) ${ }^{420,421}$, it allowed to identify the low abundant proteins of soluble fraction that would otherwise be masked in a classical shotgun mass spectrometry quantification. For example, identification of Hebp1 was not reported in the previous proteomic analyses performed on the total brain homogenate from $3 \times \mathrm{Tg}$ - $\mathrm{AD}$ mice likely due to the above-mentioned masking 260 .

The identification of peptides in shotgun mass spectrometry does not linearly correlates with the abundance of a given peptide. Besides abundance, the detection of peptides depends on several other factors which include charge, length, chemical modifications and others ${ }^{422}$. Nevertheless, it is well established that the relative quantity of specific peptide can be reliably compared between different samples based on the intensity ${ }^{423}$. Formerly, labeling techniques were frequently used for quantification of the peptides. But with the development of instrumentation and post-acquisition data analysis, label-free quantification became a reliable method bypassing the technical difficulties of labeling approaches ${ }^{424}$.

In this study, phosphoproteomic data were acquired in the DDA mode. Therefore, prior enrichment of phosphopeptides was extremely important to increase the probability of their identification by MS. Label-free quantification was also used to assess the levels of phosphosites. Label-free approach increases the number of identified phosphopeptides compared to more complex labeled samples. However, in this approach the sample preparation has to be carefully controlled since the quantification is based solely on the intensity of a single peptide. High correlation between the intensities of individual peptides in four biological replicates attest to reliability and high reproducibility of obtained data.

\subsubsection{Bioinformatic analysis}

To reliably quantify expression changes between the samples, only the proteins that were identified at least in two biological replicates at each analyzed time point were used for quantification and further analysis. At the same time, the proteins that are highly abundant in one condition (e.g. disease) and are below the detection limit in the other one were excluded from the analysis. Such proteins could potentially demonstrate the highest degree of change between $3 \times \mathrm{Tg}-\mathrm{AD}$ and control mice. However, a reliable quantification of such changes is not possible without imputation of the missing values. To avoid any potential artifacts related to the imputation, I decided to continue further data analysis without it and 
focus only on the proteins that were reliably quantified in both conditions. The full list of proteins identified exclusively in one condition (disease or control) at each time point is available in Supplementary table 4.

To interpret the proteomic data in a broader biological context, a combination of IPA analysis and GO annotation was used. Both analyses rely on the previous knowledge about individual proteins and phosphosites stored at corresponding databases. These tools provided a valuable overview of biological processes and functions affected in this study. However, such analysis is not devoid of possible shortcomings. Potential problems include incomplete databases, imprecise or incorrect annotation and redundancy ${ }^{425,426}$. Nevertheless, such analysis allows for uncovering the hidden information that could not be deconvoluted by manual annotation such as identification of upstream regulators or canonical signaling pathways.

\subsection{Conclusions and perspectives}

The results obtained in this thesis project provide a quantitative proteome map of $A D$ progression in $3 \times \mathrm{Tg}-\mathrm{AD}$ model. Several novel proteins were identified that could serve as putative presymptomatic markers of the disorder. These data can serve as a starting point to allow for a more thorough investigation of these markers in relation to their roles in AD pathogenesis. In this thesis, I took first steps to characterize one such marker, heme-binding protein 1.

Collectively, my data suggest that the elevation of Hebp1 expression may have a propathogenic role in AD. Generation of Hebp1-knockout mice would be beneficial to verify this hypothesis. Crossbreeding of such Hebp1-KO mice with 3×Tg-AD or other mouse model of AD would allow us to determine whether Hebp1 depletion can delay or ameliorate pathology on cognitive and anatomical levels. Moreover, such knockout model can shed light on the physiological function of Hebp1 in the brain and other organs.

The experiments in Hebp1-KO HeLa cells demonstrated that Hebp1 is crucial for mediation

of heme-invoked apoptosis. However, it remains elusive whether the same effect can be observed in neurons. For this purpose, I developed CRISPR/Cas9 constructs that can target 
rodent Hebp1 in primary cultured neurons from mouse and rat. Genetic knockout of Hebp1 in neurons will allow us to determine the relationship between Hebp1 and apoptosis in these cells. A further reaching step towards understanding the role of Hebp1 in AD, would be the application of neurons differentiated from AD patients' cells (induced pluripotent stem cells or fibroblasts) ${ }^{427}$. Such cell models are optimal for in vitro studies of AD mechanisms as they cover the key factors important for $\mathrm{AD}$ modeling such as an appropriate specie (human), cell type (neurons) and the disease genotype and/or phenotype ${ }^{428}$. In addition, such cells can be easily modified genetically, for example to deplete Hebp1 expression.

As mentioned in the Introduction, current biomarkers used in AD diagnosis are restricted to symptomatic onset of $\mathrm{AD}$ or $\mathrm{MCI}$ at best. Therefore, another important outcome of this study is the list of putative presymptomatic markers that can be used in the diagnosis of AD. These presymptomatic markers were identified in the brain tissue which is typically not available for diagnostic procedures. Nevertheless, these markers belong to the soluble fraction of brain proteins and thus the possibility exists that they can also be detected in CSF or plasma. Examination of these putative presymptomatic markers in CSF and plasma of AD patients with highly specific antibodies or targeted mass spectrometry techniques can determine their potential in early diagnosis of AD. 


\section{References}

1. Jorm, A. F. \& Jolley, D. The incidence of dementia: a meta-analysis. Neurology 51, 72833 (1998).

2. GBD 2015 Disease and Injury Incidence and Prevalence Collaborators, G. 2015 D. and I. I. and P. Global, regional, and national incidence, prevalence, and years lived with disability for 310 diseases and injuries, 1990-2015: a systematic analysis for the Global Burden of Disease Study 2015. Lancet (London, England) 388, 1545-1602 (2016).

3. Alzheimer's Association. 2017 Alzheimer's disease facts and figures. Alzheimer's Dement. 13, 325-373 (2017).

4. Strassnig, M. \& Ganguli, M. About a peculiar disease of the cerebral cortex: Alzheimer's original case revisited. Psychiatry (Edgmont). 2, 30-3 (2005).

5. Katzman, R. Editorial: The prevalence and malignancy of Alzheimer disease. A major killer. Arch. Neurol. 33, 217-8 (1976).

6. Katzman, R. Dementias. Postgrad. Med. 64, 119-25 (1978).

7. Vinters, H. V. Emerging Concepts in Alzheimer's Disease. Annu. Rev. Pathol. Mech. Dis. 10, 291-319 (2015).

8. Marsden, I. T., Minamide, L. S. \& Bamburg, J. R. Amyloid- $\beta$-induced amyloid- $\beta$ secretion: a possible feed-forward mechanism in Alzheimer's Disease. J. Alzheimers. Dis. 24, 68191 (2011).

9. Muller, U. C. \& Zheng, H. Physiological Functions of APP Family Proteins. Cold Spring Harb. Perspect. Med. 2, a006288-a006288 (2012).

10. Dawkins, E. \& Small, D. H. Insights into the physiological function of the $\beta$-amyloid precursor protein: beyond Alzheimer's disease. J. Neurochem. 129, 756-69 (2014).

11. Lee, J. et al. Adaptor Protein Sorting Nexin 17 Regulates Amyloid Precursor Protein Trafficking and Processing in the Early Endosomes. J. Biol. Chem. 283, 11501-11508 (2008).

12. O’Brien, R. J. \& Wong, P. C. Amyloid precursor protein processing and Alzheimer's disease. Annu. Rev. Neurosci. 34, 185-204 (2011).

13. Huse, J. T. et al. $\beta$-Secretase Processing in the Trans-Golgi Network Preferentially Generates Truncated Amyloid Species That Accumulate in Alzheimer's Disease Brain. J. Biol. Chem. 277, 16278-16284 (2002).

14. LaFerla, F. M., Green, K. N. \& Oddo, S. Intracellular amyloid- $\beta$ in Alzheimer's disease. Nat. Rev. Neurosci. 8, 499-509 (2007).

15. Soto, C., Brañes, M. C., Alvarez, J. \& Inestrosa, N. C. Structural determinants of the Alzheimer's amyloid beta-peptide. J. Neurochem. 63, 1191-8 (1994).

16. Cuello, A. C. Intracellular and extracellular Abeta, a tale of two neuropathologies. Brain Pathol. 15, 66-71 (2005). 
17. Guela, C. et al. Aging renders the brain vulnerable to amyloid $\beta$-protein neurotoxicity. Nat. Med. 4, 827-831 (1998).

18. Selkoe, D. J. \& Hardy, J. The amyloid hypothesis of Alzheimer's disease at 25 years. EMBO Mol. Med. 8, 595-608 (2016).

19. Mandelkow, E.-M. \& Mandelkow, E. Biochemistry and cell biology of tau protein in neurofibrillary degeneration. Cold Spring Harb. Perspect. Med. 2, a006247 (2012).

20. Arriagada, P. V, Growdon, J. H., Hedley-Whyte, E. T. \& Hyman, B. T. Neurofibrillary tangles but not senile plaques parallel duration and severity of Alzheimer's disease. Neurology 42, 631-9 (1992).

21. Buée, L. et al. From tau phosphorylation to tau aggregation: what about neuronal death?: Figure 1. Biochem. Soc. Trans. 38, 967-972 (2010).

22. Zempel, H. \& Mandelkow, E. Lost after translation: missorting of Tau protein and consequences for Alzheimer disease. Trends Neurosci. 37, 721-732 (2014).

23. Kolarova, M., García-Sierra, F., Bartos, A., Ricny, J. \& Ripova, D. Structure and Pathology of Tau Protein in Alzheimer Disease. Int. J. Alzheimers. Dis. 2012, 1-13 (2012).

24. Couchie, D. et al. Primary structure of high molecular weight tau present in the peripheral nervous system. Proc. Natl. Acad. Sci. U. S. A. 89, 4378-81 (1992).

25. Brandt, R. \& Lee, G. Functional organization of microtubule-associated protein tau. Identification of regions which affect microtubule growth, nucleation, and bundle formation in vitro. J. Biol. Chem. 268, 3414-9 (1993).

26. Drechsel, D. N., Hyman, A. A., Cobb, M. H. \& Kirschner, M. W. Modulation of the dynamic instability of tubulin assembly by the microtubule-associated protein tau. Mol. Biol. Cell 3, 1141-54 (1992).

27. Yagensky, O., Kalantary Dehaghi, T. \& Chua, J. J. E. The Roles of Microtubule-Based Transport at Presynaptic Nerve Terminals. Front. Synaptic Neurosci. 8, 3 (2016).

28. Hyman, B. T., Augustinack, J. C. \& Ingelsson, M. Transcriptional and conformational changes of the tau molecule in Alzheimer's disease. Biochim. Biophys. Acta - Mol. Basis Dis. 1739, 150-157 (2005).

29. Matenia, D. \& Mandelkow, E.-M. The tau of MARK: a polarized view of the cytoskeleton. Trends Biochem. Sci. 34, 332-342 (2009).

30. Grundke-Iqbal, I. et al. Abnormal phosphorylation of the microtubule-associated protein tau (tau) in Alzheimer cytoskeletal pathology. Proc. Natl. Acad. Sci. U. S. A. 83, 4913-7 (1986).

31. Wang, J.-Z., Grundke-Iqbal, I. \& Iqbal, K. Kinases and phosphatases and tau sites involved in Alzheimer neurofibrillary degeneration. Eur. J. Neurosci. 25, 59-68 (2007).

32. Tenreiro, S., Eckermann, K. \& Outeiro, T. F. Protein phosphorylation in neurodegeneration: friend or foe? Front. Mol. Neurosci. 7, 42 (2014).

33. Li, B., Chohan, M. O., Grundke-Iqbal, I. \& Iqbal, K. Disruption of microtubule network by References | 106 
Alzheimer abnormally hyperphosphorylated tau. Acta Neuropathol. 113, 501-511 (2007).

34. Alonso, A. C., Zaidi, T., Grundke-Iqbal, I. \& Iqbal, K. Role of abnormally phosphorylated tau in the breakdown of microtubules in Alzheimer disease. Proc. Natl. Acad. Sci. 91, (1994).

35. Alonso, A. del C., Grundke-Iqbal, I., Barra, H. S. \& Iqbal, K. Abnormal phosphorylation of tau and the mechanism of Alzheimer neurofibrillary degeneration: Sequestration of microtubule-associated proteins 1 and 2 and the disassembly of microtubules by the abnormal tau. Proc. Natl. Acad. Sci. 94, (1997).

36. Sperling, R. A. et al. Toward defining the preclinical stages of Alzheimer's disease: Recommendations from the National Institute on Aging-Alzheimer's Association workgroups on diagnostic guidelines for Alzheimer's disease. Alzheimer's Dement. 7, 280-292 (2011).

37. Klunk, W. E. et al. Imaging brain amyloid in Alzheimer's disease with Pittsburgh Compound-B. Ann. Neurol. 55, 306-319 (2004).

38. Jack, C. R. et al. 11C PiB and structural MRI provide complementary information in imaging of Alzheimer's disease and amnestic mild cognitive impairment. Brain 131, 665-680 (2008).

39. Rowe, C. C. et al. Amyloid imaging results from the Australian Imaging, Biomarkers and Lifestyle (AIBL) study of aging. Neurobiol. Aging 31, 1275-1283 (2010).

40. De Meyer, G. et al. Diagnosis-Independent Alzheimer Disease Biomarker Signature in Cognitively Normal Elderly People. Arch. Neurol. 67, 949 (2010).

41. Arriagada, P. V., Marzloff, K. \& Hyman, B. T. Distribution of Alzheimer-type pathologic changes in nondemented elderly individuals matches the pattern in Alzheimer's disease. 42, 1681-1688 (1992).

42. Morris, J. C. et al. Cerebral amyloid deposition and diffuse plaques in "normal" aging: Evidence for presymptomatic and very mild Alzheimer's disease. Neurology 46, 707719 (1996).

43. Mosconi, L. Glucose metabolism in normal aging and Alzheimer's disease: Methodological and physiological considerations for PET studies. Clin. Transl. imaging 1, (2013).

44. Mosconi, L. et al. Pre-clinical detection of Alzheimer's disease using FDG-PET, with or without amyloid imaging. J. Alzheimers. Dis. 20, 843-54 (2010).

45. Shen, Q. et al. Volumetric and visual rating of magnetic resonance imaging scans in the diagnosis of amnestic mild cognitive impairment and Alzheimer's disease. Alzheimers. Dement. 7, e101-8 (2011).

46. Nakamura, A. et al. High performance plasma amyloid- $\beta$ biomarkers for Alzheimer's disease. Nature (2018). doi:10.1038/nature25456

47. Olsson, B. et al. CSF and blood biomarkers for the diagnosis of Alzheimer's disease: a 
systematic review and meta-analysis. Lancet Neurol. 15, 673-684 (2016).

48. Buchhave, P. et al. Cerebrospinal Fluid Levels of $\beta$-Amyloid 1-42, but Not of Tau, Are Fully Changed Already 5 to 10 Years Before the Onset of Alzheimer Dementia. Arch. Gen. Psychiatry 69, 98 (2012).

49. Hardy, J. A. \& Higgins, G. A. Alzheimer's disease: the amyloid cascade hypothesis. Science 256, 184-5 (1992).

50. Herrup, K. The case for rejecting the amyloid cascade hypothesis. Nat. Neurosci. 18, 794-799 (2015).

51. Morris, G. P., Clark, I. A. \& Vissel, B. Inconsistencies and controversies surrounding the amyloid hypothesis of Alzheimer's disease. Acta Neuropathol. Commun. 2, 135 (2014).

52. LEE, H.-G. et al. Challenging the Amyloid Cascade Hypothesis: Senile Plaques and Amyloid- $\beta$ as Protective Adaptations to Alzheimer Disease. Ann. N. Y. Acad. Sci. 1019, 1-4 (2004).

53. Musiek, E. S. \& Holtzman, D. M. Three dimensions of the amyloid hypothesis: time, space and 'wingmen'. Nat. Neurosci. 18, 800-806 (2015).

54. Bertram, L., McQueen, M. B., Mullin, K., Blacker, D. \& Tanzi, R. E. Systematic metaanalyses of Alzheimer disease genetic association studies: the AlzGene database. Nat. Genet. 39, 17-23 (2007).

55. Esquerda-Canals, G., Montoliu-Gaya, L., Güell-Bosch, J. \& Villegas, S. Mouse Models of Alzheimer's Disease. J. Alzheimer's Dis. 57, 1171-1183 (2017).

56. Meraz-Ríos, M. A., Franco-Bocanegra, D., Toral Rios, D. \& Campos-Peña, V. Early onset Alzheimer's disease and oxidative stress. Oxid. Med. Cell. Longev. 2014, 375968 (2014).

57. Head, E., Powell, D., Gold, B. T. \& Schmitt, F. A. Alzheimer's Disease in Down Syndrome. Eur. J. Neurodegener. Dis. 1, 353-364 (2012).

58. Farrer, L. A. et al. Effects of Age, Sex, and Ethnicity on the Association Between Apolipoprotein E Genotype and Alzheimer Disease. JAMA 278, 1349 (1997).

59. Liu, C.-C., Kanekiyo, T., Xu, H. \& Bu, G. Apolipoprotein E and Alzheimer disease: risk, mechanisms and therapy. Nat. Rev. Neurol. 9, 106-118 (2013).

60. Kanekiyo, T. et al. Heparan Sulphate Proteoglycan and the Low-Density Lipoprotein Receptor-Related Protein 1 Constitute Major Pathways for Neuronal Amyloid- Uptake. J. Neurosci. 31, 1644-1651 (2011).

61. Kim, J. et al. Overexpression of Low-Density Lipoprotein Receptor in the Brain Markedly Inhibits Amyloid Deposition and Increases Extracellular $A \beta$ Clearance. Neuron 64, 632-644 (2009).

62. Chen, Y., Durakoglugil, M. S., Xian, X. \& Herz, J. ApoE4 reduces glutamate receptor function and synaptic plasticity by selectively impairing ApoE receptor recycling. Proc. Natl. Acad. Sci. U. S. A. 107, 12011-6 (2010).

63. Ji, Y. et al. Apolipoprotein E isoform-specific regulation of dendritic spine morphology References | 108 
in apolipoprotein E transgenic mice and Alzheimer's disease patients. Neuroscience 122, 305-15 (2003).

64. Keene, C. D., Cudaback, E., Li, X., Montine, K. S. \& Montine, T. J. Apolipoprotein E isoforms and regulation of the innate immune response in brain of patients with Alzheimer's disease. Curr. Opin. Neurobiol. 21, 920-928 (2011).

65. Small, G. W. et al. Apolipoprotein E type 4 allele and cerebral glucose metabolism in relatives at risk for familial Alzheimer disease. JAMA 273, 942-7

66. Narain, Y. et al. The ACE gene and Alzheimer's disease susceptibility. J. Med. Genet. 37, 695-7 (2000).

67. Cook, L. J. et al. Candidate gene association studies of the $\alpha 4$ (CHRNA4) and $\beta 2$ (CHRNB2) neuronal nicotinic acetylcholine receptor subunit genes in Alzheimer's disease. Neurosci. Lett. 358, 142-146 (2004).

68. Li, Y. et al. Association of late-onset Alzheimer's disease with genetic variation in multiple members of the GAPD gene family. Proc. Natl. Acad. Sci. U. S. A. 101, 1568893 (2004).

69. Larson, E. B. et al. Exercise is associated with reduced risk for incident dementia among persons 65 years of age and older. 144, 73-81 (2006).

70. Stern, Y. et al. Influence of Education and Occupation on the Incidence of Alzheimer's Disease. JAMA J. Am. Med. Assoc. 271, 1004 (1994).

71. Lye, T. C. \& Shores, E. A. Traumatic brain injury as a risk factor for Alzheimer's disease: A review. Neuropsychol. Rev. 10, 115-129 (2000).

72. Ahtiluoto, S. et al. Diabetes, Alzheimer disease, and vascular dementia: A populationbased neuropathologic study. Neurology 75, 1195-1202 (2010).

73. Anstey, K. J., von Sanden, C., Salim, A. \& O'Kearney, R. Smoking as a Risk Factor for Dementia and Cognitive Decline: A Meta-Analysis of Prospective Studies. Am. J. Epidemiol. 166, 367-378 (2007).

74. Whitmer, R. A. et al. Central obesity and increased risk of dementia more than three decades later. Neurology 71, 1057-1064 (2008).

75. Kivipelto, M. et al. Obesity and Vascular Risk Factors at Midlife and the Risk of Dementia and Alzheimer Disease. Arch. Neurol. 62, 1556-1560 (2005).

76. Ninomiya, T. et al. Midlife and Late-Life Blood Pressure and Dementia in Japanese Elderly: The Hisayama Study. Hypertension 58, 22-28 (2011).

77. Lin, M. T. \& Beal, M. F. Mitochondrial dysfunction and oxidative stress in neurodegenerative diseases. Nature 443, 787-795 (2006).

78. Chan, D. C. Mitochondria: Dynamic Organelles in Disease, Aging, and Development. Cell 125, 1241-1252 (2006).

79. Burté, F., Carelli, V., Chinnery, P. F. \& Yu-Wai-Man, P. Disturbed mitochondrial dynamics and neurodegenerative disorders. Nat. Rev. Neurol. 11, 11-24 (2015). 
80. Manczak, M., Calkins, M. J. \& Reddy, P. H. Impaired mitochondrial dynamics and abnormal interaction of amyloid beta with mitochondrial protein Drp1 in neurons from patients with Alzheimer's disease: implications for neuronal damage. Hum. Mol. Genet. 20, 2495-2509 (2011).

81. Zanna, C. et al. OPA1 mutations associated with dominant optic atrophy impair oxidative phosphorylation and mitochondrial fusion. Brain 131, 352-367 (2008).

82. Schon, E. A. \& Area-Gomez, E. Mitochondria-associated ER membranes in Alzheimer disease. Mol. Cell. Neurosci. 55, 26-36 (2013).

83. Area-Gomez, E. et al. Upregulated function of mitochondria-associated ER membranes in Alzheimer disease. EMBO J. 31, 4106-4123 (2012).

84. Hedskog, L. et al. Modulation of the endoplasmic reticulum-mitochondria interface in Alzheimer's disease and related models. Proc. Natl. Acad. Sci. 110, 7916-7921 (2013).

85. Sorrentino, V. et al. Enhancing mitochondrial proteostasis reduces amyloid- $\beta$ proteotoxicity. Nature 552, 187 (2017).

86. Vives-Bauza, C. et al. PINK1-dependent recruitment of Parkin to mitochondria in mitophagy. Proc. Natl. Acad. Sci. 107, 378-383 (2010).

87. Du, F. et al. PINK1 signalling rescues amyloid pathology and mitochondrial dysfunction in Alzheimer's disease. Brain 140, 3233-3251 (2017).

88. Cenini, G., Rub, C., Bruderek, M. \& Voos, W. Amyloid- $\beta$ peptides interfere with mitochondrial preprotein import competence by a coaggregation process. Mol. Biol. Cell 27, 3257-3272 (2016).

89. Mossmann, D. et al. Amyloid- $\beta$ Peptide Induces Mitochondrial Dysfunction by Inhibition of Preprotein Maturation. Cell Metab. 20, 662-669 (2014).

90. Ott, M., Gogvadze, V., Orrenius, S. \& Zhivotovsky, B. Mitochondria, oxidative stress and cell death. Apoptosis 12, 913-922 (2007).

91. Nunomura, A. et al. Oxidative damage is the earliest event in Alzheimer disease. J. Neuropathol. Exp. Neurol. 60, 759-67 (2001).

92. Praticò, D., Uryu, K., Leight, S., Trojanoswki, J. Q. \& Lee, V. M. Increased lipid peroxidation precedes amyloid plaque formation in an animal model of Alzheimer amyloidosis. J. Neurosci. 21, 4183-7 (2001).

93. Johnson, W. M., Wilson-Delfosse, A. L. \& Mieyal, J. J. Dysregulation of Glutathione Homeostasis in Neurodegenerative Diseases. Nutrients 4, 1399-1440 (2012).

94. Velliquette, R. A., O'Connor, T. \& Vassar, R. Energy Inhibition Elevates -Secretase Levels and Activity and Is Potentially Amyloidogenic in APP Transgenic Mice: Possible Early Events in Alzheimer's Disease Pathogenesis. J. Neurosci. 25, 10874-10883 (2005).

95. Lovell, M. A., Xiong, S., Xie, C., Davies, P. \& Markesbery, W. R. Induction of hyperphosphorylated tau in primary rat cortical neuron cultures mediated by oxidative stress and glycogen synthase kinase-3. J. Alzheimers. Dis. 6, 659-71-81 
(2004).

96. Price, J. L. et al. Neuron number in the entorhinal cortex and CA1 in preclinical Alzheimer disease. Arch. Neurol. 58, 1395-402 (2001).

97. Hof, P. R. et al. Stereologic evidence for persistence of viable neurons in layer II of the entorhinal cortex and the CA1 field in Alzheimer disease. J. Neuropathol. Exp. Neurol. 62, 55-67 (2003).

98. Theofilas, P. et al. Probing the correlation of neuronal loss, neurofibrillary tangles, and cell death markers across the Alzheimer's disease Braak stages: a quantitative study in humans. Neurobiol. Aging 61, 1-12 (2018).

99. Gorman, A. M. Neuronal cell death in neurodegenerative diseases: recurring themes around protein handling. J. Cell. Mol. Med. 12, 2263-80 (2008).

100. Cotman, C. W. \& Su, J. H. Mechanisms of neuronal death in Alzheimer's disease. Brain Pathol. 6, 493-506 (1996).

101. Manczak, M. et al. Mitochondria are a direct site of $A \beta$ accumulation in Alzheimer's disease neurons: implications for free radical generation and oxidative damage in disease progression. Hum. Mol. Genet. 15, 1437-1449 (2006).

102. Lustbader, J. W. et al. ABAD Directly Links A to Mitochondrial Toxicity in Alzheimer's Disease. Science (80-. ). 304, 448-452 (2004).

103. Bartley, M. G. et al. Overexpression of amyloid- $\beta$ protein precursor induces mitochondrial oxidative stress and activates the intrinsic apoptotic cascade. J. Alzheimers. Dis. 28, 855-68 (2012).

104. Cha, M.-Y. et al. Mitochondria-Specific Accumulation of Amyloid $\beta$ Induces Mitochondrial Dysfunction Leading to Apoptotic Cell Death. PLoS One 7, e34929 (2012).

105. Du, H. et al. Cyclophilin D deficiency attenuates mitochondrial and neuronal perturbation and ameliorates learning and memory in Alzheimer's disease. Nat. Med. 14, 1097-1105 (2008).

106. Lakshmana, M. K. et al. A fragment of the scaffolding protein RanBP9 is increased in Alzheimer's disease brains and strongly potentiates amyloid- $\beta$ peptide generation. FASEB J. 24, 119-127 (2010).

107. Klamt, F. et al. Oxidant-induced apoptosis is mediated by oxidation of the actinregulatory protein cofilin. Nat. Cell Biol. 11, 1241-1246 (2009).

108. Meamar, R. et al. Enalapril protects endothelial cells against induced apoptosis in Alzheimer's disease. J. Res. Med. Sci. 18, S1-5 (2013).

109. Fossati, S., Ghiso, J. \& Rostagno, A. TRAIL death receptors DR4 and DR5 mediate cerebral microvascular endothelial cell apoptosis induced by oligomeric Alzheimer's Aß. Cell Death Dis. 3, e321 (2012).

110. Rohn, T. T. et al. Lack of Pathology in a Triple Transgenic Mouse Model of Alzheimer's 
Disease after Overexpression of the Anti-Apoptotic Protein Bcl-2.J. Neurosci. 28, 30513059 (2008).

111. Diaz-Gerevini, G. T. et al. Beneficial action of resveratrol: How and why? Nutrition 32, 174-178 (2016).

112. Deepmala et al. Clinical trials of N-acetylcysteine in psychiatry and neurology: A systematic review. Neurosci. Biobehav. Rev. 55, 294-321 (2015).

113. Wyss-Coray, T. \& Rogers, J. Inflammation in Alzheimer disease-a brief review of the basic science and clinical literature. Cold Spring Harb. Perspect. Med. 2, a006346 (2012).

114. Li, Q. \& Barres, B. A. Microglia and macrophages in brain homeostasis and disease. Nat. Rev. Immunol. (2017). doi:10.1038/nri.2017.125

115. Bolmont, T. et al. Dynamics of the Microglial/Amyloid Interaction Indicate a Role in Plaque Maintenance. J. Neurosci. 28, 4283-4292 (2008).

116. Cagnin, A. et al. In-vivo measurement of activated microglia in dementia. Lancet 358, 461-467 (2001).

117. Edison, P. et al. Microglia, amyloid, and cognition in Alzheimer's disease: An [11C](R)PK11195-PET and [11C]PIB-PET study. Neurobiol. Dis. 32, 412-419 (2008).

118. Allen, N. J. \& Eroglu, C. Cell Biology of Astrocyte-Synapse Interactions. Neuron 96, 697708 (2017).

119. Bélanger, M. \& Magistretti, P. J. The role of astroglia in neuroprotection. Dialogues Clin. Neurosci. 11, 281-95 (2009).

120. Wyss-Coray, T. et al. Adult mouse astrocytes degrade amyloid- $\beta$ in vitro and in situ. Nat. Med. 9, 453-457 (2003).

121. Mott, R. T. et al. Neuronal expression of CD22: Novel mechanism for inhibiting microglial proinflammatory cytokine production. Glia 46, 369-379 (2004).

122. Hosokawa, M., Klegeris, A., Maguire, J. \& McGeer, P. L. Expression of complement messenger RNAs and proteins by human oligodendroglial cells. Glia 42, 417-423 (2003).

123. Walker, D. G., Lue, L. F. \& Beach, T. G. Gene expression profiling of amyloid beta peptidestimulated human post-mortem brain microglia. Neurobiol. Aging 22, 957-66

124. Chen, X. et al. RAGE: a potential target for Abeta-mediated cellular perturbation in Alzheimer's disease. Curr. Mol. Med. 7, 735-42 (2007).

125. Chakrabarty, P. et al. Massive gliosis induced by interleukin-6 suppresses A $\beta$ deposition in vivo: evidence against inflammation as a driving force for amyloid deposition. FASEB J. 24, 548-559 (2010).

126. He, P. et al. Deletion of tumor necrosis factor death receptor inhibits amyloid $\beta$ generation and prevents learning and memory deficits in Alzheimer's mice. J. Cell Biol. 178, 829-841 (2007). 
127. Emmerling, M. R., Watson, M. D., Raby, C. A. \& Spiegel, K. The role of complement in Alzheimer's disease pathology. Biochim. Biophys. Acta 1502, 158-71 (2000).

128. Fraser, D. A., Pisalyaput, K. \& Tenner, A. J. C1q enhances microglial clearance of apoptotic neurons and neuronal blebs, and modulates subsequent inflammatory cytokine production. J. Neurochem. 112, 733-743 (2010).

129. Stevens, B. et al. The Classical Complement Cascade Mediates CNS Synapse Elimination. Cell 131, 1164-1178 (2007).

130. Landreth, G. E. \& Reed-Geaghan, E. G. in Current topics in microbiology and immunology 336, 137-153 (2009).

131. McGeer, P. L., Schulzer, M. \& McGeer, E. G. Arthritis and anti-inflammatory agents as possible protective factors for Alzheimer's disease: a review of 17 epidemiologic studies. Neurology 47, 425-32 (1996).

132. Nathan, C. et al. Protection from Alzheimer's-like disease in the mouse by genetic ablation of inducible nitric oxide synthase. J. Exp. Med. 202, 1163-1169 (2005).

133. Ulrich, J. D., Ulland, T. K., Colonna, M. \& Holtzman, D. M. Elucidating the Role of TREM2 in Alzheimer's Disease. Neuron 94, 237-248 (2017).

134. Yamada, M. Cerebral amyloid angiopathy: emerging concepts. J. stroke 17, 17-30 (2015).

135. Yamada, M. Risk factors for cerebral amyloid angiopathy in the elderly. Ann. N. Y. Acad. Sci. 977, 37-44 (2002).

136. Hartz, A. M. S. et al. Amyloid- Contributes to Blood-Brain Barrier Leakage in Transgenic Human Amyloid Precursor Protein Mice and in Humans With Cerebral Amyloid Angiopathy. Stroke 43, 514-523 (2012).

137. Cordonnier, C. et al. Prevalence and severity of microbleeds in a memory clinic setting. Neurology 66, 1356-1360 (2006).

138. Henneman, W. J. P. et al. MRI Biomarkers of Vascular Damage and Atrophy Predicting Mortality in a Memory Clinic Population. Stroke 40, 492-498 (2009).

139. Wyss-Coray, T. Ageing, neurodegeneration and brain rejuvenation. Nature 539, 180186 (2016).

140. Viles, J. H. Metal ions and amyloid fiber formation in neurodegenerative diseases. Copper, zinc and iron in Alzheimer's, Parkinson's and prion diseases. Coord. Chem. Rev. 256, 2271-2284 (2012).

141. Jian Dong, $\neq$ et al. Metal Binding and Oxidation of Amyloid- $\beta$ within Isolated Senile Plaque Cores: Raman Microscopic Evidence†. (2003). doi:10.1021/BI0272151

142. Sarell, C. J., Wilkinson, S. R. \& Viles, J. H. Substoichiometric Levels of $\mathrm{Cu}^{2+}$ Ions Accelerate the Kinetics of Fiber Formation and Promote Cell Toxicity of Amyloid- $\beta$ from Alzheimer Disease. J. Biol. Chem. 285, 41533-41540 (2010).

143. Hureau, C. \& Faller, P. A $\beta$-mediated ROS production by $\mathrm{Cu}$ ions: Structural insights, 
mechanisms and relevance to Alzheimer's disease. Biochimie 91, 1212-1217 (2009).

144. Tomljenovic, L. Aluminum and Alzheimer's disease: after a century of controversy, is there a plausible link? J. Alzheimers. Dis. 23, 567-98 (2011).

145. Kim, H. J., Khalimonchuk, O., Smith, P. M. \& Winge, D. R. Structure, function, and assembly of heme centers in mitochondrial respiratory complexes. Biochim. Biophys. Acta - Mol. Cell Res. 1823, 1604-1616 (2012).

146. Schipper, H. M., Cissé, S. \& Stopa, E. G. Expression of heme oxygenase-1 in the senescent and alzheimer-diseased brain. Ann. Neurol. 37, 758-768 (1995).

147. Atamna, H. \& Frey, W. H. A role for heme in Alzheimer's disease: heme binds amyloid beta and has altered metabolism. Proc. Natl. Acad. Sci. U. S. A. 101, 11153-8 (2004).

148. Owen, J. E., Bishop, G. M. \& Robinson, S. R. Uptake and Toxicity of Hemin and Iron in Cultured Mouse Astrocytes. Neurochem. Res. 41, 298-306 (2016).

149. Gemelli, C., Dongmo, B. M., Ferrarini, F., Grande, A. \& Corsi, L. Cytotoxic effect of hemin in colonic epithelial cell line: Involvement of $18 \mathrm{kDa}$ translocator protein (TSPO). Life Sci. 107, 14-20 (2014).

150. Atamna, H. \& Boyle, K. Amyloid-beta peptide binds with heme to form a peroxidase: Relationship to the cytopathologies of Alzheimer's disease. Proc. Natl. Acad. Sci. 103, 3381-3386 (2006).

151. Ghosh, C., Seal, M., Mukherjee, S. \& Ghosh Dey, S. Alzheimer's Disease: A Heme-A $\beta$ Perspective. Acc. Chem. Res. 48, 2556-2564 (2015).

152. Chiziane, E. et al. Free Heme and Amyloid- $\beta$ : A Fatal Liaison in Alzheimer's Disease. J. Alzheimer's Dis. 61, 963-984 (2018).

153. Sasaki, N. et al. Advanced Glycation End Products in Alzheimer's Disease and Other Neurodegenerative Diseases. Am. J. Pathol. 153, 1149-1155 (1998).

154. Zare-Shahabadi, A., Masliah, E., Johnson, G. V. W. \& Rezaei, N. Autophagy in Alzheimer's disease. Rev. Neurosci. 26, 385-95 (2015).

155. Sheng, M., Sabatini, B. L. \& Südhof, T. C. Synapses and Alzheimer's disease. Cold Spring Harb. Perspect. Biol. 4, (2012).

156. Davies, P. \& Maloney, A. J. Selective loss of central cholinergic neurons in Alzheimer's disease. Lancet (London, England) 2, 1403 (1976).

157. Birks, J. \& Harvey, R. J. Donepezil for dementia due to Alzheimer's disease. Cochrane Database Syst. Rev. CD001190 (2006). doi:10.1002/14651858.CD001190.pub2

158. Birks, J. S. \& Grimley Evans, J. in Cochrane Database of Systematic Reviews (ed. Birks, J. S.) CD001191 (John Wiley \& Sons, Ltd, 2015). doi:10.1002/14651858.CD001191.pub3

159. Ankarcrona, M. et al. Glutamate-induced neuronal death: a succession of necrosis or apoptosis depending on mitochondrial function. Neuron 15, 961-73 (1995).

160. Schneider, L. S., Dagerman, K. S., Higgins, J. P. T. \& McShane, R. Lack of Evidence for the References | 114 
Efficacy of Memantine in Mild Alzheimer Disease. Arch. Neurol. 68, 991 (2011).

161. Tariot, P. N. \& Federoff, H. J. Current treatment for Alzheimer disease and future prospects. Alzheimer Dis. Assoc. Disord. 17 Suppl 4, S105-13

162. McShane, R., Areosa Sastre, A. \& Minakaran, N. Memantine for dementia. Cochrane Database Syst. Rev. CD003154 (2006). doi:10.1002/14651858.CD003154.pub5

163. De Strooper, B., Vassar, R. \& Golde, T. The secretases: enzymes with therapeutic potential in Alzheimer disease. Nat. Rev. Neurol. 6, 99-107 (2010).

164. Schor, N. F. What the halted phase III $\gamma$-secretase inhibitor trial may (or may not) be telling us. Ann. Neurol. 69, 237-239 (2011).

165. Green, R. C. et al. Effect of Tarenflurbil on Cognitive Decline and Activities of Daily Living in Patients With Mild Alzheimer Disease: A Randomized Controlled Trial. JAMA 302, 2557 (2009).

166. Schenk, D. et al. Immunization with amyloid- $\beta$ attenuates Alzheimer-disease-like pathology in the PDAPP mouse. Nature 400, 173-177 (1999).

167. Gilman, S. et al. Clinical effects of A immunization (AN1792) in patients with AD in an interrupted trial. Neurology 64, 1553-1562 (2005).

168. Schroeter, S. et al. Immunotherapy Reduces Vascular Amyloid- $\beta$ in PDAPP Mice. $J$. Neurosci. 28, 6787-6793 (2008).

169. Vandenberghe, R. et al. Bapineuzumab for mild to moderate Alzheimer's disease in two global, randomized, phase 3 trials. Alzheimers. Res. Ther. 8, 18 (2016).

170. Salloway, S. et al. A phase 2 randomized trial of ELND005, scyllo-inositol, in mild to moderate Alzheimer disease. Neurology 77, 1253-1262 (2011).

171. Cherny, R. A. et al. Treatment with a copper-zinc chelator markedly and rapidly inhibits beta-amyloid accumulation in Alzheimer's disease transgenic mice. Neuron 30, 66576 (2001).

172. Brunden, K. R., Trojanowski, J. Q. \& Lee, V. M.-Y. Advances in tau-focused drug discovery for Alzheimer's disease and related tauopathies. Nat. Rev. Drug Discov. 8, 783-793 (2009).

173. Taniguchi, S. et al. Inhibition of Heparin-induced Tau Filament Formation by Phenothiazines, Polyphenols, and Porphyrins. J. Biol. Chem. 280, 7614-7623 (2005).

174. Coulibaly, B. et al. Strong Gametocytocidal Effect of Methylene Blue-Based Combination Therapy against Falciparum Malaria: A Randomised Controlled Trial. PLoS One 4, e5318 (2009).

175. Schirmer, R. H., Adler, H., Pickhardt, M. \& Mandelkow, E. 'Lest we forget you methylene blue ...'. Neurobiol. Aging 32, 2325.e7-2325.e16 (2011).

176. Wischik, C. M., Bentham, P., Wischik, D. J. \& Seng, K. M. 03-04-07: Tau aggregation inhibitor (TAI) therapy with rember ${ }^{\mathrm{TM}}$ arrests disease progression in mild and moderate Alzheimer's disease over 50 weeks. Alzheimer's Dement. 4, T167 (2008). 
177. Huang, Y. \& Mucke, L. Alzheimer mechanisms and therapeutic strategies. Cell 148, 1204-22 (2012).

178. Brunden, K. R. et al. Epothilone D Improves Microtubule Density, Axonal Integrity, and Cognition in a Transgenic Mouse Model of Tauopathy. J. Neurosci. 30, 13861-13866 (2010).

179. Panza, F. et al. Tau-Centric Targets and Drugs in Clinical Development for the Treatment of Alzheimer's Disease. Biomed Res. Int. 2016, 3245935 (2016).

180. Huang, Y. Abeta-independent roles of apolipoprotein E4 in the pathogenesis of Alzheimer's disease. Trends Mol. Med. 16, 287-94 (2010).

181. Locatelli, S. et al. Reduction of plasma 24S-hydroxycholesterol (cerebrosterol) levels using high-dosage simvastatin in patients with hypercholesterolemia: evidence that simvastatin affects cholesterol metabolism in the human brain. Arch. Neurol. 59, 2136 (2002).

182. Sano, M. et al. A randomized, double-blind, placebo-controlled trial of simvastatin to treat Alzheimer disease. Neurology 77, 556-563 (2011).

183. Hori, Y. et al. A Food and Drug Administration-approved Asthma Therapeutic Agent Impacts Amyloid $\beta$ in the Brain in a Transgenic Model of Alzheimer Disease. J. Biol. Chem. 290, 1966-1978 (2015).

184. Holmes, C. et al. Systemic inflammation and disease progression in Alzheimer disease. Neurology 73, 768-774 (2009).

185. Cuadrado-Tejedor, M. \& García-Osta, A. Current animal models of Alzheimer's disease: challenges in translational research. Front. Neurol. 5, 182 (2014).

186. Schultz, C. et al. Filamentous tau pathology in nerve cells, astrocytes, and oligodendrocytes of aged baboons. J. Neuropathol. Exp. Neurol. 59, 39-52 (2000).

187. Sani, S. et al. Distribution, progression and chemical composition of cortical amyloidbeta deposits in aged rhesus monkeys: similarities to the human. Acta Neuropathol. 105, 145-56 (2003).

188. Cummings, B. J., Su, J. H., Cotman, C. W., White, R. \& Russell, M. J. Beta-amyloid accumulation in aged canine brain: a model of early plaque formation in Alzheimer's disease. Neurobiol. Aging 14, 547-60

189. Head, E. et al. $\beta$-Amyloid deposition and tau phosphorylation in clinically characterized aged cats. Neurobiol. Aging 26, 749-763 (2005).

190. Uchida, K. et al. Senile Plaques and Other Senile Changes in the Brain of an Aged American Black Bear. Vet. Pathol. 32, 412-414 (1995).

191. Braak, H., Braak, E. \& Strothjohann, M. Abnormally phosphorylated tau protein related to the formation of neurofibrillary tangles and neuropil threads in the cerebral cortex of sheep and goat. Neurosci. Lett. 171, 1-4 (1994).

192. Van Dam, D. \& De Deyn, P. P. Animal models in the drug discovery pipeline for 
Alzheimer's disease. Br. J. Pharmacol. 164, 1285-300 (2011).

193. Moran, P. M. Differential effects of scopolamine and mecamylamine on working and reference memory in the rat. Pharmacol. Biochem. Behav. 45, 533-8 (1993).

194. Estapé, N. \& Steckler, T. Cholinergic blockade impairs performance in operant DNMTP in two inbred strains of mice. Pharmacol. Biochem. Behav. 72, 319-34 (2002).

195. Lescaudron, L. \& Stein, D. G. Differences in memory impairment and response to GM1 ganglioside treatment following electrolytic or ibotenic acid lesions of the nucleus basalis magnocellularis. Restor. Neurol. Neurosci. 15, 25-37 (1999).

196. Mulder, J. et al. Galantamine-induced behavioral recovery after sublethal excitotoxic lesions to the rat medial septum. Behav. Brain Res. 163, 33-41 (2005).

197. Sloan, H. L., Good, M. \& Dunnett, S. B. Double dissociation between hippocampal and prefrontal lesions on an operant delayed matching task and a water maze reference memory task. Behav. Brain Res. 171, 116-126 (2006).

198. Castañé, A., Theobald, D. E. H. \& Robbins, T. W. Selective lesions of the dorsomedial striatum impair serial spatial reversal learning in rats. Behav. Brain Res. 210, 74-83 (2010).

199. Glenn, M. J., Nesbitt, C. \& Mumby, D. G. Perirhinal cortex lesions produce variable patterns of retrograde amnesia in rats. Behav. Brain Res. 141, 183-93 (2003).

200. Hauss-Wegrzyniak, B., Dobrzanski, P., Stoehr, J. D. \& Wenk, G. L. Chronic neuroinflammation in rats reproduces components of the neurobiology of Alzheimer's disease. Brain Res. 780, 294-303 (1998).

201. Szabados, T. et al. A chronic Alzheimer's model evoked by mitochondrial poison sodium azide for pharmacological investigations. Behav. Brain Res. 154, 31-40 (2004).

202. Yamada, M. et al. Implanted cannula-mediated repetitive administration of A $\beta 25-35$ into the mouse cerebral ventricle effectively impairs spatial working memory. Behav. Brain Res. 164, 139-146 (2005).

203. Sipos, E. et al. $\beta$-Amyloid pathology in the entorhinal cortex of rats induces memory deficits: Implications for Alzheimer's disease. Neuroscience 147, 28-36 (2007).

204. Saito, T., Matsuba, Y., Yamazaki, N., Hashimoto, S. \& Saido, T. C. Calpain Activation in Alzheimer's Model Mice Is an Artifact of APP and Presenilin Overexpression. J. Neurosci. 36, 9933-6 (2016).

205. Neve, R. L. \& Robakis, N. K. Alzheimer's disease: a re-examination of the amyloid hypothesis. Trends Neurosci. 21, 15-19 (1998).

206. Dyrks, T., Dyrks, E., Masters, C. L. \& Beyreuther, K. Amyloidogenicity of rodent and human beta A4 sequences. FEBS Lett. 324, 231-6 (1993).

207. Quon, D. et al. Formation of $\beta$-amyloid protein deposits in brains of transgenic mice. Nature 352, 239-241 (1991).

208. Lamb, B. T. et al. Introduction and expression of the 400 kilobase precursor amyloid 
protein gene in transgenic mice. Nat. Genet. 5, 22-30 (1993).

209. Games, D. et al. Alzheimer-type neuropathology in transgenic mice overexpressing V717F $\beta$-amyloid precursor protein. Nature 373, 523-527 (1995).

210. Hsiao, K. et al. Correlative memory deficits, Abeta elevation, and amyloid plaques in transgenic mice. Science 274, 99-102 (1996).

211. Sturchler-Pierrat, C. et al. Two amyloid precursor protein transgenic mouse models with Alzheimer disease-like pathology. Proc. Natl. Acad. Sci. U. S. A. 94, 13287-92 (1997).

212. Hyman, B. T., Tanzi, R. E., Marzloff, K., Barbour, R. \& Schenk, D. Kunitz protease inhibitor-containing amyloid beta protein precursor immunoreactivity in Alzheimer's disease. J. Neuropathol. Exp. Neurol. 51, 76-83 (1992).

213. Mucke, L. et al. High-level neuronal expression of abeta 1-42 in wild-type human amyloid protein precursor transgenic mice: synaptotoxicity without plaque formation. J. Neurosci. 20, 4050-8 (2000).

214. Rockenstein, E., Mallory, M., Mante, M., Sisk, A. \& Masliaha, E. Early formation of mature amyloid-beta protein deposits in a mutant APP transgenic model depends on levels of Abeta(1-42). J. Neurosci. Res. 66, 573-82 (2001).

215. Chishti, M. A. et al. Early-onset amyloid deposition and cognitive deficits in transgenic mice expressing a double mutant form of amyloid precursor protein 695. J. Biol. Chem. 276, 21562-70 (2001).

216. Clark, R. F. et al. The structure of the presenilin 1 (S182) gene and identification of six novel mutations in early onset AD families. Nat. Genet. 11, 219-222 (1995).

217. Citron, M. et al. Mutant presenilins of Alzheimer's disease increase production of 42residue amyloid beta-protein in both transfected cells and transgenic mice. Nat. Med. 3, 67-72 (1997).

218. Guo, Q. et al. Increased vulnerability of hippocampal neurons to excitotoxic necrosis in presenilin-1 mutant knock-in mice. Nat. Med. 5, 101-106 (1999).

219. Duff, K. et al. Increased amyloid- $\beta 42(43)$ in brains of mice expressing mutant presenilin 1. Nature 383, 710-713 (1996).

220. Wang, R., Dineley, K. ., Sweatt, J. . \& Zheng, H. Presenilin 1 familial Alzheimer's disease mutation leads to defective associative learning and impaired adult neurogenesis. Neuroscience 126, 305-312 (2004).

221. Holcomb, L. et al. Accelerated Alzheimer-type phenotype in transgenic mice carrying both mutant amyloid precursor protein and presenilin 1 transgenes. Nat. Med. 4, 97100 (1998).

222. Flood, D. G. et al. FAD mutant PS-1 gene-targeted mice: increased A beta 42 and A beta deposition without APP overproduction. Neurobiol. Aging 23, 335-48

223. Oakley, H. et al. Intraneuronal beta-Amyloid Aggregates, Neurodegeneration, and 
Neuron Loss in Transgenic Mice with Five Familial Alzheimer's Disease Mutations: Potential Factors in Amyloid Plaque Formation. J. Neurosci. 26, 10129-10140 (2006).

224. Sasaguri, H. et al. APP mouse models for Alzheimer's disease preclinical studies. EMBO J. 36, 2473-2487 (2017).

225. Raber, J. et al. Isoform-specific effects of human apolipoprotein E on brain function revealed in ApoE knockout mice: increased susceptibility of females. Proc. Natl. Acad. Sci. U. S. A. 95, 10914-9 (1998).

226. Pankiewicz, J. E. et al. Blocking the apoE/A $\beta$ interaction ameliorates A $\beta$-related pathology in APOE $\varepsilon 2$ and $\varepsilon 4$ targeted replacement Alzheimer model mice. Acta Neuropathol. Commun. 2, 75 (2014).

227. Graybeal, J. J. et al. Human ApoE $\varepsilon 4$ alters circadian rhythm activity, IL-1 $\beta$, and GFAP in CRND8 mice. J. Alzheimers. Dis. 43, 823-34 (2015).

228. Arancio, 0. et al. RAGE potentiates $A \beta$-induced perturbation of neuronal function in transgenic mice. EMBO J. 23, 4096-4105 (2004).

229. Wang, Y. et al. TREM2 Lipid Sensing Sustains the Microglial Response in an Alzheimer's Disease Model. Cell 160, 1061-1071 (2015).

230. Pluci ska, K. et al. Knock-In of Human BACE1 Cleaves Murine APP and Reiterates Alzheimer-like Phenotypes. J. Neurosci. 34, 10710-10728 (2014).

231. Colton, C. A. et al. The effects of NOS2 gene deletion on mice expressing mutated human AbetaPP. J. Alzheimers. Dis. 15, 571-87 (2008).

232. Sagare, A. P. et al. Pericyte loss influences Alzheimer-like neurodegeneration in mice. Nat. Commun. 4, 2932 (2013).

233. Oddo, S. et al. Triple-transgenic model of Alzheimer's disease with plaques and tangles: intracellular Abeta and synaptic dysfunction. Neuron 39, 409-21 (2003).

234. Hutton, M. et al. Association of missense and $5^{\prime}$-splice-site mutations in tau with the inherited dementia FTDP-17. Nature 393, 702-705 (1998).

235. Oddo, S., Caccamo, A., Kitazawa, M., Tseng, B. P. \& LaFerla, F. M. Amyloid deposition precedes tangle formation in a triple transgenic model of Alzheimer's disease. Neurobiol. Aging 24, 1063-70 (2003).

236. Stover, K. R., Campbell, M. A., Van Winssen, C. M. \& Brown, R. E. Early detection of cognitive deficits in the 3xTg-AD mouse model of Alzheimer's disease. Behav. Brain Res. 289, 29-38 (2015).

237. Caruso, D. et al. Age-related changes in neuroactive steroid levels in 3xTg-AD mice. Neurobiol. Aging 34, 1080-9 (2013).

238. Schena, M., Shalon, D., Davis, R. W. \& Brown, P. O. Quantitative monitoring of gene expression patterns with a complementary DNA microarray. Science 270, 467-70 (1995).

239. Trevino, V., Falciani, F. \& Barrera-Saldaña, H. A. DNA microarrays: a powerful genomic 
tool for biomedical and clinical research. Mol. Med. 13, 527-41 (2007).

240. Blalock, E. M., Buechel, H. M., Popovic, J., Geddes, J. W. \& Landfield, P. W. Microarray analyses of laser-captured hippocampus reveal distinct gray and white matter signatures associated with incipient Alzheimer's disease. J. Chem. Neuroanat. 42, 118126 (2011).

241. Silva, A. R. T. et al. Transcriptional Alterations Related to Neuropathology and Clinical Manifestation of Alzheimer's Disease. PLoS One 7, e48751 (2012).

242. Hokama, M. et al. Altered Expression of Diabetes-Related Genes in Alzheimer's Disease Brains: The Hisayama Study. Cereb. Cortex 24, 2476-2488 (2014).

243. Holtman, I. R. et al. Induction of a common microglia gene expression signature by aging and neurodegenerative conditions: a co-expression meta-analysis. Acta Neuropathol. Commun. 3, 31 (2015).

244. Orre, M. et al. Isolation of glia from Alzheimer's mice reveals inflammation and dysfunction. Neurobiol. Aging 35, 2746-2760 (2014).

245. Eberwine, J., Sul, J.-Y., Bartfai, T. \& Kim, J. The promise of single-cell sequencing. Nat. Methods 11, 25-27 (2014).

246. Mathys, H. et al. Temporal Tracking of Microglia Activation in Neurodegeneration at Single-Cell Resolution. Cell Rep. 21, 366-380 (2017).

247. Keren-Shaul, H. et al. A Unique Microglia Type Associated with Restricting Development of Alzheimer's Disease. Cell 169, 1276-1290.e17 (2017).

248. Alkallas, R., Fish, L., Goodarzi, H. \& Najafabadi, H. S. Inference of RNA decay rate from transcriptional profiling highlights the regulatory programs of Alzheimer's disease. Nat. Commun. 8, 909 (2017).

249. Satoh, J., Kino, Y. \& Niida, S. MicroRNA-Seq Data Analysis Pipeline to Identify Blood Biomarkers for Alzheimer's Disease from Public Data. Biomark. Insights 10, BMI.S25132 (2015).

250. Chen, G., Qiu, C., Zhang, Q., Liu, B. \& Cui, Q. Genome-Wide Analysis of Human SNPs at Long Intergenic Noncoding RNAs. Hum. Mutat. 34, 338-344 (2013).

251. Patrick, E. et al. Dissecting the role of non-coding RNAs in the accumulation of amyloid and tau neuropathologies in Alzheimer's disease. Mol. Neurodegener. 12, 51 (2017).

252. Magistri, M., Velmeshev, D., Makhmutova, M. \& Faghihi, M. A. Transcriptomics Profiling of Alzheimer's Disease Reveal Neurovascular Defects, Altered Amyloid- $\beta$ Homeostasis, and Deregulated Expression of Long Noncoding RNAs. J. Alzheimers. Dis. 48, 647-65 (2015).

253. Gunaratne, J. et al. Extensive mass spectrometry-based analysis of the fission yeast proteome: the Schizosaccharomyces pombe PeptideAtlas. Mol. Cell. Proteomics 12, 1741-51 (2013).

254. EDMAN, P. A method for the determination of amino acid sequence in peptides. Arch. 
Biochem. 22, 475 (1949).

255. Brune, D. C. et al. ABRF ESRG 2006 study: Edman sequencing as a method for polypeptide quantitation. J. Biomol. Tech. 18, 306-20 (2007).

256. Yates, J. R. The Revolution and Evolution of Shotgun Proteomics for Large-Scale Proteome Analysis. J. Am. Chem. Soc. 135, 1629-1640 (2013).

257. Picotti, P. \& Aebersold, R. Selected reaction monitoring-based proteomics: workflows, potential, pitfalls and future directions. Nat. Methods 9, 555-566 (2012).

258. Chou, J. L. et al. Early dysregulation of the mitochondrial proteome in a mouse model of Alzheimer's disease. J. Proteomics 74, 466-479 (2011).

259. Rhein, V. et al. Amyloid- and tau synergistically impair the oxidative phosphorylation system in triple transgenic Alzheimer's disease mice. Proceedings of the National Academy of Sciences 106, 20057-20062 (2009).

260. Ciavardelli, D. et al. Alterations of brain and cerebellar proteomes linked to $A \beta$ and tau pathology in a female triple-transgenic murine model of Alzheimer's disease. Cell Death and Disease 1, (2010).

261. Savas, J. N. et al. Amyloid Accumulation Drives Proteome-wide Alterations in Mouse Models of Alzheimer's Disease-like Pathology. Cell Rep. 21, 2614-2627 (2017).

262. Korolainen, M. A., Nyman, T. A., Aittokallio, T. \& Pirttilä, T. An update on clinical proteomics in Alzheimer's research. J. Neurochem. 112, 1386-1414 (2010).

263. Chang, R. Y. K., Nouwens, A. S., Dodd, P. R. \& Etheridge, N. The synaptic proteome in Alzheimer's disease. Alzheimer's Dement. 9, 499-511 (2013).

264. Chang, R. Y. K., Etheridge, N., Dodd, P. R. \& Nouwens, A. S. Targeted quantitative analysis of synaptic proteins in Alzheimer's disease brain. Neurochem. Int. 75, 66-75 (2014).

265. Zhou, J. et al. Proteomic analysis of postsynaptic density in Alzheimer's Disease. Clin. Chim. Acta 420, 62-68 (2013).

266. Bai, B. et al. U1 small nuclear ribonucleoprotein complex and RNA splicing alterations in Alzheimer's disease. Proc. Natl. Acad. Sci. U. S. A. 110, 16562-7 (2013).

267. Portelius, E. et al. Proteomic studies of cerebrospinal fluid biomarkers of Alzheimer's disease: an update. Expert Rev. Proteomics 14, 1007-1020 (2017).

268. Ringman, J. M. et al. Proteomic Changes in Cerebrospinal Fluid of Presymptomatic and Affected Persons Carrying Familial Alzheimer Disease Mutations. Arch. Neurol. 69, 96 (2012).

269. Street, J. M. et al. Identification and proteomic profiling of exosomes in human cerebrospinal fluid. J. Transl. Med. 10, 5 (2012).

270. Thambisetty, M. et al. Association of Plasma Clusterin Concentration With Severity, Pathology, and Progression in Alzheimer Disease. Arch. Gen. Psychiatry 67, 739 (2010).

271. Oddo, S. The ubiquitin-proteasome system in Alzheimer's disease. J. Cell. Mol. Med. 12, 
363-73 (2008).

272. Schedin-Weiss, S., Winblad, B. \& Tjernberg, L. O. The role of protein glycosylation in Alzheimer disease. FEBS J. 281, 46-62 (2014).

273. Hunter, T. Protein kinases and phosphatases: The Yin and Yang of protein phosphorylation and signaling. Cell 80, 225-236 (1995).

274. Riley, N. M. \& Coon, J. J. Phosphoproteomics in the Age of Rapid and Deep Proteome Profiling. Anal. Chem. 88, 74-94 (2016).

275. Dammer, E. B. et al. Quantitative phosphoproteomics of Alzheimer's disease reveals cross-talk between kinases and small heat shock proteins. Proteomics 15, 508-519 (2015).

276. Triplett, J. C., Swomley, A. M., Cai, J., Klein, J. B. \& Butterfield, D. A. Quantitative Phosphoproteomic Analyses of the Inferior Parietal Lobule from Three Different Pathological Stages of Alzheimer's Disease. J. Alzheimer's Dis. 49, 45-62 (2015).

277. Tagawa, K. et al. Comprehensive phosphoproteome analysis unravels the core signaling network that initiates the earliest synapse pathology in preclinical Alzheimer's disease brain. Hum. Mol. Genet. 24, 540-558 (2015).

278. Huttner, W. B., Schiebler, W., Greengard, P. \& De Camilli, P. Synapsin I (protein I), a nerve terminal-specific phosphoprotein. III. Its association with synaptic vesicles studied in a highly purified synaptic vesicle preparation. J. Cell Biol. 96, 1374-88 (1983).

279. Humphrey, S. J., Azimifar, S. B. \& Mann, M. High-throughput phosphoproteomics reveals in vivo insulin signaling dynamics. Nat. Biotechnol. 33, 990-5 (2015).

280. Cox, J. \& Mann, M. MaxQuant enables high peptide identification rates, individualized p.p.b.-range mass accuracies and proteome-wide protein quantification. Nat. Biotechnol. 26, 1367-72 (2008).

281. Cox, J. et al. Andromeda: a peptide search engine integrated into the MaxQuant environment. J. Proteome Res. 10, 1794-805 (2011).

282. Linding, R. et al. Systematic Discovery of In Vivo Phosphorylation Networks. Cell 129, 1415-1426 (2007).

283. Zafar, S. et al. Strain-Specific Altered Regulatory Response of Rab7a and Tau in Creutzfeldt-Jakob Disease and Alzheimer's Disease. Mol. Neurobiol. 54, 697-709 (2017).

284. Braak, H. \& Braak, E. Neuropathological stageing of Alzheimer-related changes. Acta Neuropathol. 82, 239-59 (1991).

285. Grau-Rivera, O. et al. Clinicopathological Correlations and Concomitant Pathologies in Rapidly Progressive Dementia: A Brain Bank Series. Neurodegener. Dis. 15, 350-360 (2015).

286. Yang, P.-C. \& Mahmood, T. Western blot: Technique, theory, and trouble shooting. $N$. 
Am. J. Med. Sci. 4, 429 (2012).

287. Rabe, T. I. et al. The transcription factor Uncx4.1 acts in a short window of midbrain dopaminergic neuron differentiation. Neural Dev. 7, 39 (2012).

288. Martinez Hernandez, A. et al. The diphenylpyrazole compound anle138b blocks A $\beta$ channels and rescues disease phenotypes in a mouse model for amyloid pathology. EMBO Mol. Med. 10, 32-47 (2018).

289. Frezza, C., Cipolat, S. \& Scorrano, L. Organelle isolation: functional mitochondria from mouse liver, muscle and cultured filroblasts. Nat. Protoc. 2, 287-295 (2007).

290. Shevchenko, A. et al. A strategy for identifying gel-separated proteins in sequence databases by MS alone. Biochem. Soc. Trans. 24, 893-6 (1996).

291. Atamna, H., Brahmbhatt, M., Atamna, W., Shanower, G. A. \& Dhahbi, J. M. ApoHRP-based assay to measure intracellular regulatory heme. Metallomics 7, 309-21 (2015).

292. Schindelin, J. et al. Fiji: an open-source platform for biological-image analysis. Nat. Methods 9, 676-682 (2012).

293. Wirths, O., Dins, A. \& Bayer, T. A. AßPP Accumulation and/or Intraneuronal Amyloid- $\beta$ Accumulation? The 3xTg-AD Mouse Model Revisited. J. Alzheimer's Dis. 28, 897-904 (2012).

294. Végh, M. J. et al. Hippocampal extracellular matrix levels and stochasticity in synaptic protein expression increase with age and are associated with age-dependent cognitive decline. Mol. Cell. Proteomics 13, 2975-85 (2014).

295. Sato, Y. \& Endo, T. Alteration of brain glycoproteins during aging. Geriatr. Gerontol. Int. 10, S32-S40 (2010).

296. Palop, J. J. et al. Aberrant Excitatory Neuronal Activity and Compensatory Remodeling of Inhibitory Hippocampal Circuits in Mouse Models of Alzheimer's Disease. Neuron 55, 697-711 (2007).

297. Dephoure, N., Gould, K. L., Gygi, S. P. \& Kellogg, D. R. Mapping and analysis of phosphorylation sites: a quick guide for cell biologists. Mol. Biol. Cell 24, 535-42 (2013).

298. McCracken, S., Lambermon, M. \& Blencowe, B. J. SRm160 splicing coactivator promotes transcript 3'-end cleavage. Mol. Cell. Biol. 22, 148-60 (2002).

299. Ren, J. et al. DOG 1.0: illustrator of protein domain structures. Cell Res. 19, 271-273 (2009).

300. Saura, C. A. \& Valero, J. The role of CREB signaling in Alzheimer's disease and other cognitive disorders. Rev. Neurosci. 22, 153-169 (2011).

301. Salmina, A. B. et al. H2S- and NO-Signaling Pathways in Alzheimer's Amyloid Vasculopathy: Synergism or Antagonism? Front. Physiol. 6, 361 (2015).

302. Chen, Y. et al. Alzheimer's -Secretase (BACE1) Regulates the cAMP/PKA/CREB Pathway Independently of -Amyloid. J. Neurosci. 32, 11390-11395 (2012). 
303. Li, H. et al. cAMP/PKA signaling pathway contributes to neuronal apoptosis via regulating IDE expression in a mixed model of type 2 diabetes and Alzheimer's disease. J. Cell. Biochem. 119, 1616-1626 (2018).

304. Liu, S.-L. et al. The Role of Cdk5 in Alzheimer's Disease. Mol. Neurobiol. 53, 4328-4342 (2016).

305. Colwill, K. et al. The Clk/Sty protein kinase phosphorylates SR splicing factors and regulates their intranuclear distribution. EMBO J. 15, 265-75 (1996).

306. Chen, F. et al. Role for glyoxalase I in Alzheimer's disease. Proc. Natl. Acad. Sci. U. S. A. 101, 7687-7692 (2004).

307. More, S. S., Vartak, A. P. \& Vince, R. Restoration of Glyoxalase Enzyme Activity Precludes Cognitive Dysfunction in a Mouse Model of Alzheimer's Disease. ACS Chem. Neurosci. 4, 330-338 (2013).

308. Mueller, C. et al. The heme degradation pathway is a promising serum biomarker source for the early detection of Alzheimer's disease. J. Alzheimers. Dis. 19, 1081-91 (2010).

309. Liu, H., Luo, K. \& Luo, D. Guanosine monophosphate reductase 1 is a potential therapeutic target for Alzheimer's disease. Sci. Rep. 8, 2759 (2018).

310. Yamada, T., Kawamata, T., Walker, D. G. \& McGeer, P. L. Vimentin immunoreactivity in normal and pathological human brain tissue. Acta Neuropathol. 84, 157-162 (1992).

311. Wang, Q. et al. Proteomic analysis of neurofibrillary tangles in Alzheimer disease identifies GAPDH as a detergent-insoluble paired helical filament tau binding protein. FASEB J. 19, 869-871 (2005).

312. Gal, J. et al. Detergent Insoluble Proteins and Inclusion Body-Like Structures Immunoreactive for PRKDC/DNA-PK/DNA-PKcs, FTL, NNT, and AIFM1 in the Amygdala of Cognitively Impaired Elderly Persons. J. Neuropathol. Exp. Neurol. 77, 2139 (2018).

313. Kong, W. et al. The Construction of Common and Specific Significance Subnetworks of Alzheimer's Disease from Multiple Brain Regions. Biomed Res. Int. 2015, 1-13 (2015).

314. Saczynski, J. S., White, L., Peila, R. L., Rodriguez, B. L. \& Launer, L. J. The Relation between Apolipoprotein A-I and Dementia: The Honolulu-Asia Aging Study. Am. J. Epidemiol. 165, 985-992 (2007).

315. Merched, A., Xia, Y., Visvikis, S., Serot, J. . \& Siest, G. Decreased high-density lipoprotein cholesterol and serum apolipoprotein AI concentrations are highly correlated with the severity of Alzheimer's disease. Neurobiol. Aging 21, 27-30 (2000).

316. Hoos, M. D. et al. Longitudinal study of differential protein expression in an Alzheimer's mouse model lacking inducible nitric oxide synthase. J. Proteome Res. 12, 4462-77 (2013).

317. Kaiser, M. et al. Progressive loss of a glial potassium channel (KCNJ10) in the spinal cord of the SOD1 (G93A) transgenic mouse model of amyotrophic lateral sclerosis. J. 
Neurochem. 99, 900-912 (2006).

318. Drummond, E. et al. Proteomic differences in amyloid plaques in rapidly progressive and sporadic Alzheimer's disease. Acta Neuropathol. 133, 933-954 (2017).

319. Zafar, S. et al. Prion Protein Interactome: Identifying Novel Targets in Slowly and Rapidly Progressive Forms of Alzheimer's Disease. J. Alzheimer's Dis. 59, 265-275 (2017).

320. Taketani, S., Adachi, Y., Ishii, T. \& Chem, J. B. Molecular Characterization of a Newly Identified Heme-binding Protein Induced during Differentiation of urine Molecular Characterization of a Newly Identified Heme-binding Protein Induced during Differentiation of urine Eryth. 273, 31388-31394 (1998).

321. Blackmon, B. J., Dailey, T. A., Lianchun, X. \& Dailey, H. A. Characterization of a human and mouse tetrapyrrole-binding protein. Arch. Biochem. Biophys. 407, 196-201 (2002).

322. Dias, J. S. et al. The first structure from the SOUL/HBP family of heme-binding proteins, murine P22HBP. J. Biol. Chem. 281, 31553-31561 (2006).

323. Atamna, H. Heme, iron, and the mitochondrial decay of ageing. Ageing Res. Rev. 3, 303318 (2004).

324. Szigeti, A. et al. Induction of necrotic cell death and mitochondrial permeabilization by heme binding protein 2/SOUL. FEBS Lett. 580, 6447-6454 (2006).

325. Szigeti, A. et al. Facilitation of mitochondrial outer and inner membrane permeabilization and cell death in oxidative stress by a novel Bcl-2 homology 3 domain protein. J. Biol. Chem. 285, 2140-2151 (2010).

326. Fortunato, A. E., Sordino, P. \& Andreakis, N. Evolution of the SOUL Heme-Binding Protein Superfamily Across Eukarya. J. Mol. Evol. 82, 279-290 (2016).

327. Hung, V. et al. Proteomic mapping of cytosol-facing outer mitochondrial and ER membranes in living human cells by proximity biotinylation. Elife 6, e24463 (2017).

328. Hung, V. et al. Proteomic mapping of the human mitochondrial intermembrane space in live cells via ratiometric APEX tagging. Mol. Cell 55, 332-41 (2014).

329. Calvo, S. E., Clauser, K. R. \& Mootha, V. K. MitoCarta2.0: an updated inventory of mammalian mitochondrial proteins. Nucleic Acids Res. 44, D1251-D1257 (2016).

330. Zhao, W. et al. Tert-butyl hydroperoxide (t-BHP) induced apoptosis and necroptosis in endothelial cells: Roles of NOX4 and mitochondrion. Redox Biol. 11, 524 (2017).

331. Belmokhtar, C. A., Hillion, J. \& Ségal-Bendirdjian, E. Staurosporine induces apoptosis through both caspase-dependent and caspase-independent mechanisms. Oncogene 20, 3354-3362 (2001).

332. Alonso, A. C., Grundke-Iqbal, I. \& Iqbal, K. Alzheimer's disease hyperphosphorylated tau sequesters normal tau into tangles of filaments and disassembles microtubules. Nat. Med. 2, 783-7 (1996). 
333. Illenberger, S. et al. Phosphorylation of microtubule-associated proteins MAP2 and MAP4 by the protein kinase p110mark. Phosphorylation sites and regulation of microtubule dynamics. J. Biol. Chem. 271, 10834-43 (1996).

334. Itoh, T. J., Hisanaga, S., Hosoi, T., Kishimoto, T. \& Hotani, H. Phosphorylation States of Microtubule-Associated Protein 2 (MAP2) Determine the Regulatory Role of MAP2 in Microtubule Dynamics. Biochemistry 36, 12574-12582 (1997).

335. Halpain, S. \& Dehmelt, L. The MAP1 family of microtubule-associated proteins. Genome Biol. 7, 224 (2006).

336. Morsch, R., Simon, W. \& Coleman, P. D. Neurons may live for decades with neurofibrillary tangles. J. Neuropathol. Exp. Neurol. 58, 188-97 (1999).

337. Alonso, A. d. C., Zaidi, T., Novak, M., Grundke-Iqbal, I. \& Iqbal, K. Hyperphosphorylation induces self-assembly of into tangles of paired helical filaments/straight filaments. Proc. Natl. Acad. Sci. 98, 6923-6928 (2001).

338. Oddo, S. et al. Genetically augmenting tau levels does not modulate the onset or progression of A $\beta$ pathology in transgenic mice.J. Neurochem. 102, 1053-1063 (2007).

339. Andorfer, C. et al. Hyperphosphorylation and aggregation of tau in mice expressing normal human tau isoforms. J. Neurochem. 86, 582-590 (2003).

340. Ashe, K. H. \& Zahs, K. R. Probing the biology of Alzheimer's disease in mice. Neuron 66, 631-45 (2010).

341. Verstraelen, P. et al. Dysregulation of Microtubule Stability Impairs Morphofunctional Connectivity in Primary Neuronal Networks. Front. Cell. Neurosci. 11, 173 (2017).

342. Gatta, E. et al. Evidence for an imbalance between tau O-GlcNAcylation and phosphorylation in the hippocampus of a mouse model of Alzheimer's disease. Pharmacol. Res. 105, 186-197 (2016).

343. Kam, T.-I. et al. Fc $\gamma$ RIIb-SHIP2 axis links $A \beta$ to tau pathology by disrupting phosphoinositide metabolism in Alzheimer's disease model. Elife 5, (2016).

344. Onishi, T. et al. Early-onset cognitive deficits and axonal transport dysfunction in P301S mutant tau transgenic mice. Neurosci. Res. 80, 76-85 (2014).

345. Henkins, K. M. et al. Extensive p-Tau Pathology and SDS-Stable p-Tau Oligomers in Alzheimer's Cortical Synapses. Brain Pathol. 22, 826-833 (2012).

346. Lasagna-Reeves, C. A. et al. Identification of oligomers at early stages of tau aggregation in Alzheimer's disease. FASEB J. 26, 1946-1959 (2012).

347. Jicha, G. A. et al. A conformation- and phosphorylation-dependent antibody recognizing the paired helical filaments of Alzheimer's disease. J. Neurochem. 69, 2087-95 (1997).

348. Gu, G. J. et al. Role of Individual MARK Isoforms in Phosphorylation of Tau at Ser262 in Alzheimer's Disease. NeuroMolecular Med. 15, 458-469 (2013).

349. Mondragón-Rodríguez, S., Perry, G., Luna-Muñoz, J., Acevedo-Aquino, M. C. \& Williams, S. Phosphorylation of tau protein at sites Ser(396-404) is one of the earliest events in 
Alzheimer's disease and Down syndrome. Neuropathol. Appl. Neurobiol. 40, 121-135 (2014).

350. Noble, W. et al. Cdk5 is a key factor in tau aggregation and tangle formation in vivo. Neuron 38, 555-65 (2003).

351. Merino-Serrais, P. et al. The influence of phospho-tau on dendritic spines of cortical pyramidal neurons in patients with Alzheimer's disease. Brain 136, 1913-1928 (2013).

352. Salton, M., Lerenthal, Y., Wang, S.-Y., Chen, D. J. \& Shiloh, Y. Involvement of Matrin 3 and SFPQ/NONO in the DNA damage response. Cell Cycle 9, 1568-1576 (2010).

353. Zhang, Z. \& Carmichael, G. G. The Fate of dsRNA in the Nucleus: A p54nrb-Containing Complex Mediates the Nuclear Retention of Promiscuously A-to-I Edited RNAs. Cell 106, 465-476 (2001).

354. Passacantilli, I., Frisone, P., De Paola, E., Fidaleo, M. \& Paronetto, M. P. hnRNPM guides an alternative splicing program in response to inhibition of the PI3K/AKT/mTOR pathway in Ewing sarcoma cells. Nucleic Acids Res. 45, 12270-12284 (2017).

355. Fei, T. et al. Genome-wide CRISPR screen identifies HNRNPL as a prostate cancer dependency regulating RNA splicing. Proc. Natl. Acad. Sci. 114, 201617467 (2017).

356. Shehadeh, L. A. et al. SRRM2, a Potential Blood Biomarker Revealing High Alternative Splicing in Parkinson's Disease. PLoS One 5, e9104 (2010).

357. Lee, K.-Y. et al. Elevated neuronal Cdc2-like kinase activity in the Alzheimer disease brain. Neurosci. Res. 34, 21-29 (1999).

358. Liu, F. \& Gong, C.-X. Tau exon 10 alternative splicing and tauopathies. Mol. Neurodegener. 3, 8 (2008).

359. Jain, P. et al. Human CDC2-like kinase 1 (CLK1): a novel target for Alzheimer's disease. Curr. Drug Targets 15, 539-50 (2014).

360. De Jonghe, C. et al. Aberrant splicing in the presenilin-1 intron 4 mutation causes presenile Alzheimer's disease by increased Abeta42 secretion. Hum. Mol. Genet. 8, 1529-40 (1999).

361. Berson, A. et al. Cholinergic-associated loss of hnRNP-A/B in Alzheimer's disease impairs cortical splicing and cognitive function in mice. EMBO Mol. Med. 4, 730 (2012).

362. Gurel, B. et al. Early Stage Alterations in CA1 Extracellular Region Proteins Indicate Dysregulation of IL6 and Iron Homeostasis in the 5XFAD Alzheimer's Disease Mouse Model. J. Alzheimer's Dis. 61, 1399-1410 (2018).

363. Abraham, C. R., Selkoe, D. J. \& Potter, H. Immunochemical identification of the serine protease inhibitor alpha 1-antichymotrypsin in the brain amyloid deposits of Alzheimer's disease. Cell 52, 487-501 (1988).

364. Ho, G. J., Smirnova, I. V, Akaaboune, M., Hantaï, D. \& Festoff, B. W. Serine proteases and their serpin inhibitors in Alzheimer's disease. Biomed. Pharmacother. 48, 296-304 
(1994).

365. Mukhina, I. V., Korotchenko, S. A. \& Dityatev, A. E. Extracellular matrix molecules, their receptors, and extracellular proteases as synaptic plasticity modulators. Neurochem. J. 6, 89-99 (2012).

366. Gatto, M. et al. Serpins, Immunity and Autoimmunity: Old Molecules, New Functions. Clin. Rev. Allergy Immunol. 45, 267-280 (2013).

367. Bird, P. I. Serpins and regulation of cell death. Results Probl. Cell Differ. 24, 63-89 (1998).

368. Hu, J. et al. Serine protease inhibitor A3K protects rabbit corneal endothelium from barrier function disruption induced by TNF- $\alpha$. Investigative Ophthalmology and Visual Science 54, 5400-5407 (2013).

369. Lin, Z. et al. Serine protease inhibitor A3K suppressed the formation of ocular surface squamous metaplasia in a mouse model of experimental dry eye. Investig. Ophthalmol. Vis. Sci. 55, 5813-5820 (2014).

370. Zhang, B. \& Ma, J. X. SERPINA3K prevents oxidative stress induced necrotic cell death by inhibiting calcium overload. PLoS One 3, (2008).

371. Kato, K. et al. Serine proteinase inhibitor 3 and murinoglobulin I are potent inhibitors of neuropsin in adult mouse brain. J. Biol. Chem. 276, 14562-71 (2001).

372. Blacker, D. et al. Alpha-2 macroglobulin is genetically associated with Alzheimer disease. Nat. Genet. 19, 357-360 (1998).

373. Varma, V. R. et al. Alpha-2 macroglobulin in Alzheimer's disease: a marker of neuronal injury through the RCAN1 pathway. Mol. Psychiatry 22, 13-23 (2017).

374. Barcelona, P. F. \& Saragovi, H. U. A Pro-Nerve Growth Factor (proNGF) and NGF Binding Protein, $\alpha 2$-Macroglobulin, Differentially Regulates p75 and TrkA Receptors and Is Relevant to Neurodegeneration Ex Vivo and In Vivo. Mol. Cell. Biol. 35, 3396-3408 (2015).

375. Castellano, J. M. et al. Human umbilical cord plasma proteins revitalize hippocampal function in aged mice. Nature 544, 488-492 (2017).

376. Donev, R., Kolev, M., Millet, B. \& Thome, J. Neuronal death in Alzheimer's disease and therapeutic opportunities. J. Cell. Mol. Med. 13, 4329-4348 (2009).

377. Wirths, 0. \& Bayer, T. A. Neuron loss in transgenic mouse models of Alzheimer's disease. Int. J. Alzheimers. Dis. 2010, (2010).

378. Poirel, O. et al. Moderate decline in select synaptic markers in the prefrontal cortex (BA9) of patients with Alzheimer's disease at various cognitive stages. Sci. Rep. 8, 938 (2018).

379. Xie, H. et al. Rapid cell death is preceded by amyloid plaque-mediated oxidative stress. Proc. Natl. Acad. Sci. 110, 7904-7909 (2013).

380. Zhao, Y. et al. Appoptosin-Mediated Caspase Cleavage of Tau Contributes to References $\mid 128$ 
Progressive Supranuclear Palsy Pathogenesis. Neuron 87, 963-975 (2015).

381. Rissman, R. A. et al. Caspase-cleavage of tau is an early event in Alzheimer disease tangle pathology. J. Clin. Invest. 114, 121-30 (2004).

382. Scahill, R. I., Schott, J. M., Stevens, J. M., Rossor, M. N. \& Fox, N. C. Mapping the evolution of regional atrophy in Alzheimer's disease: Unbiased analysis of fluid-registered serial MRI. Proc. Natl. Acad. Sci. 99, 4703-4707 (2002).

383. Harner, M. et al. The mitochondrial contact site complex, a determinant of mitochondrial architecture. EMBO J. 30, 4356-4370 (2011).

384. Xie, J., Marusich, M. F., Souda, P., Whitelegge, J. \& Capaldi, R. A. The mitochondrial inner membrane protein Mitofilin exists as a complex with SAM50, metaxins 1 and 2, coiledcoil-helix coiled-coil-helix domain-containing protein 3 and 6 and DnaJC11. FEBS Lett. 581, 3545-3549 (2007).

385. Matsuhashi, T. et al. Mitochonic Acid 5 (MA-5) Facilitates ATP Synthase Oligomerization and Cell Survival in Various Mitochondrial Diseases. EBioMedicine 20, 27-38 (2017).

386. Ott, C. et al. Sam50 functions in mitochondrial intermembrane space bridging and biogenesis of respiratory complexes. Mol. Cell. Biol. 32, 1173-88 (2012).

387. Ding, C. et al. Mitofilin and CHCHD6 physically interact with Sam50 to sustain cristae structure. Sci. Rep. 5, 16064 (2015).

388. Kozjak-Pavlovic, V. The MICOS complex of human mitochondria. Cell Tissue Res. 367, 83-93 (2017).

389. Wiedemann, N. et al. Machinery for protein sorting and assembly in the mitochondrial outer membrane. Nature 424, 565-571 (2003).

390. Roise, D. \& Schatz, G. Mitochondrial presequences. J. Biol. Chem. 263, 4509-11 (1988).

391. Hamza, I. \& Dailey, H. A. One ring to rule them all: Trafficking of heme and heme synthesis intermediates in the metazoans. Biochim. Biophys. Acta - Mol. Cell Res. 1823, 1617-1632 (2012).

392. Ponka, P., Sheftel, A. D., English, A. M., Bohle, D. S. \& Garcia-Santos, D. Do Mammalian Cells Really Need to Export and Import Heme? Trends Biochem. Sci. 42, 395-406 (2017).

393. Klatt, P. et al. Characterization of heme-deficient neuronal nitric-oxide synthase reveals a role for heme in subunit dimerization and binding of the amino acid substrate and tetrahydrobiopterin. J. Biol. Chem. 271, 7336-42 (1996).

394. Yang, R. et al. Mitofilin regulates cytochrome c release during apoptosis by controlling mitochondrial cristae remodeling. Biochem. Biophys. Res. Commun. 428, 93-98 (2012).

395. Hettiarachchi, N. et al. Heme oxygenase-1 protects against Alzheimer's amyloid- $\beta 1-42-$ induced toxicity via carbon monoxide production. Cell Death Dis. 5, e1569-e1569 (2014). 
396. Yamaguchi, T., Komoda, Y. \& Nakajima, H. Biliverdin-IX alpha reductase and biliverdinIX beta reductase from human liver. Purification and characterization. J. Biol. Chem. 269, 24343-8 (1994).

397. Smith, M. A. et al. Heme oxygenase-1 is associated with the neurofibrillary pathology of Alzheimer's disease. Am. J. Pathol. 145, 42-7 (1994).

398. Barone, E. et al. Biliverdin reductase-A protein levels and activity in the brains of subjects with Alzheimer disease and mild cognitive impairment. Biochim. Biophys. Acta - Mol. Basis Dis. 1812, 480-487 (2011).

399. Ghiso, J., Tomidokoro, Y., Revesz, T., Frangione, B. \& Rostagno, A. CEREBRAL AMYLOID ANGIOPATHY AND ALZHEIMER'S DISEASE. Hirosaki Igaku 61, S111-S124 (2010).

400. Li, J.-G. \& Praticò, D. High levels of homocysteine results in cerebral amyloid angiopathy in mice. J. Alzheimers. Dis. 43, 29-35 (2015).

401. Natté, R. et al. Dementia in hereditary cerebral hemorrhage with amyloidosis-Dutch type is associated with cerebral amyloid angiopathy but is independent of plaques and neurofibrillary tangles. Ann. Neurol. 50, 765-72 (2001).

402. Mattson, M. P. Apoptosis in neurodegenerative disorders. Nat. Rev. Mol. Cell Biol. 1, $120-130$ (2000).

403. Fossati, S. et al. Differential activation of mitochondrial apoptotic pathways by vasculotropic amyloid- $\beta$ variants in cells composing the cerebral vessel walls. FASEB J. 24, 229-241 (2010).

404. Gao, J.-L. et al. F2L, a Peptide Derived from Heme-Binding Protein, Chemoattracts Mouse Neutrophils by Specifically Activating Fpr2, the Low-Affinity N-Formylpeptide Receptor. J. Immunol. 178, 1450-1456 (2007).

405. Devosse, T. et al. Processing of HEBP1 by Cathepsin D Gives Rise to F2L, the Agonist of Formyl Peptide Receptor 3. J. Immunol. 187, 1475-1485 (2011).

406. Cui, Y. H. et al. Up-regulation of FPR2, a chemotactic receptor for amyloid beta 1-42 (A beta 42), in murine microglial cells by TNF alpha. Neurobiol. Dis. 10, 366-77 (2002).

407. Le, Y. et al. Amyloid (beta) 42 activates a G-protein-coupled chemoattractant receptor, FPR-like-1. J. Neurosci. 21, RC123 (2001).

408. Hondius, D. C. et al. Profiling the human hippocampal proteome at all pathologic stages of Alzheimer's disease. Alzheimer's Dement. 12, 654-668 (2016).

409. Simón, A.-M. et al. Overexpression of wild-type human APP in mice causes cognitive deficits and pathological features unrelated to A $\beta$ levels. Neurobiol. Dis. 33, 369-378 (2009).

410. Cheng, N., Jiao, S., Gumaste, A., Bai, L. \& Belluscio, L. APP Overexpression Causes A Independent Neuronal Death through Intrinsic Apoptosis Pathway. eNeuro 3, (2016).

411. Saito, T. et al. Single App knock-in mouse models of Alzheimer's disease. Nat. Neurosci. 17, 661-663 (2014). 
412. Gunawardena, S. \& Goldstein, L. S. Disruption of axonal transport and neuronal viability by amyloid precursor protein mutations in Drosophila. Neuron 32, 389-401 (2001).

413. Nhan, H. S., Chiang, K. \& Koo, E. H. The multifaceted nature of amyloid precursor protein and its proteolytic fragments: friends and foes. Acta Neuropathol. 129, 1-19 (2015).

414. Richardson, A. et al. Use of Transgenic Mice in Aging Research. ILAR J. 38, 124-136 (1997).

415. van Hoesen, G. W., Hyman, B. T. \& Damasio, A. R. Entorhinal cortex pathology in Alzheimer's disease. Hippocampus 1, 1-8 (1991).

416. Bonner-Jackson, A., Mahmoud, S., Miller, J. \& Banks, S. J. Verbal and non-verbal memory and hippocampal volumes in a memory clinic population. Alzheimers. Res. Ther. 7, 61 (2015).

417. Jacobs, H. I. L. et al. The cerebellum in Alzheimer's disease: evaluating its role in cognitive decline. Brain 141, 37-47 (2018).

418. Lee, J. H., Ryan, J., Andreescu, C., Aizenstein, H. \& Lim, H. K. Brainstem morphological changes in Alzheimer's disease. Neuroreport 26, 411-5 (2015).

419. Graumann, J., Scheltema, R. A., Zhang, Y., Cox, J. \& Mann, M. A framework for intelligent data acquisition and real-time database searching for shotgun proteomics. Mol. Cell. Proteomics 11, M111.013185 (2012).

420. Bereczki, E. et al. Synaptic markers of cognitive decline in neurodegenerative diseases: a proteomic approach. Brain 141, 582-595 (2018).

421. Bereczki, E. et al. Synaptic proteins predict cognitive decline in Alzheimer's disease and Lewy body dementia. Alzheimer's Dement. 12, 1149-1158 (2016).

422. Brönstrup, M. Absolute quantification strategies in proteomics based on mass spectrometry. Expert Rev. Proteomics 1, 503-512 (2004).

423. Kuster, B., Schirle, M., Mallick, P. \& Aebersold, R. Scoring proteomes with proteotypic peptide probes. Nat. Rev. Mol. Cell Biol. 6, 577-583 (2005).

424. Cox, J. et al. Accurate Proteome-wide Label-free Quantification by Delayed Normalization and Maximal Peptide Ratio Extraction, Termed MaxLFQ. Mol. Cell. Proteomics 13, 2513-2526 (2014).

425. Gaudet, P. \& Dessimoz, C. Gene Ontology: Pitfalls, Biases, and Remedies. Methods Mol. Biol. 1446, 189-205 (2017).

426. Khatri, P. \& Drăghici, S. Ontological analysis of gene expression data: current tools, limitations, and open problems. Bioinformatics 21, 3587-95 (2005).

427. Arber, C., Lovejoy, C. \& Wray, S. Stem cell models of Alzheimer's disease: progress and challenges. Alzheimers. Res. Ther. 9, 42 (2017).

428. Israel, M. A. et al. Probing sporadic and familial Alzheimer's disease using induced pluripotent stem cells. Nature 482, 216-220 (2012). 


\section{Appendices}

\subsection{List of abbreviations}

\begin{tabular}{|c|c|}
\hline$\left({ }^{11} \mathrm{C}\right) \mathrm{PIB}$ & (11)C-labelled Pittsburgh Compound-B ligand \\
\hline $3 \times \mathrm{Tg}-\mathrm{AD}$ & Triple-transgenic mouse model of Alzheimer's disease \\
\hline Acan & Aggrecan core protein \\
\hline ACN & Acetonitrile \\
\hline $\mathrm{AD}$ & Alzheimer's disease \\
\hline ALS & Amyotrophic lateral sclerosis \\
\hline ANOVA & Analysis of variance \\
\hline Apoa1 & Apolipoprotein A1 \\
\hline APOE & Apolipoprotein E \\
\hline APOJ & Apolipoprotein J (clustering) \\
\hline APP & Amyloid precursor protein \\
\hline Atp2b4 & ATPase Plasma Membrane $\mathrm{Ca}^{2+}$ Transporting 4 \\
\hline $\mathrm{A} \beta$ & Amyloid beta \\
\hline Bax & bcl-2-like protein 4 \\
\hline BBB & blood brain barrier \\
\hline Bcl-2 & B-cell lymphoma 2 protein \\
\hline Blvrb & Biliverdin reductase B \\
\hline BSA & Bovine serum albumin \\
\hline $\mathrm{C} 1 \mathrm{qc}$ & Complement C1q subcomponent subunit C \\
\hline CA1 & Carbonic anhydrase 1 \\
\hline CAA & Cerebral amyloid angiopathy \\
\hline CaMKI/II & $\mathrm{Ca}^{2+} /$ calmodulin-dependent kinase I and II \\
\hline cAMP & Cyclic adenosine monophosphate \\
\hline Cdk5 & Cyclin-dependent-like kinase 5 \\
\hline cDNA & Complementary deoxyribonucleic acid \\
\hline CHRNB2 & Cholinergic receptor nicotinic beta 2 subunit \\
\hline CK2 & Casein kinase II \\
\hline CLK & Cdc2-like kinase \\
\hline CNS & Central nervous system \\
\hline Co-IP & Co-immunoprecipitation \\
\hline COX2 & Cyclooxygenase 2 \\
\hline Cox4 & Cyclooxygenase 4 \\
\hline $\mathrm{CPO}$ & Coproporphyrinogen oxidase \\
\hline CREB & Cyclic AMP-responsive element-binding protein 1 \\
\hline CSF & Cerebrospinal fluid \\
\hline Ctip2 & Protein Ctip2 \\
\hline Ctpd & Cathepsin D \\
\hline DAPI & 4',6-diamidino-2-phenylindole \\
\hline DDA & data-dependent acquisition \\
\hline DIV & Days in vitro \\
\hline DMEM & Dulbeccos Modified Eagles Medium \\
\hline DNA & Deoxyribonucleic acid \\
\hline
\end{tabular}




$\begin{array}{ll}\text { Dpysl4 } & \text { Dihydropyrimidinase Like 4 } \\ \text { DR4/5 } & \text { Death receptor 4/5 } \\ \text { DTT } & \text { Dithiothreitol } \\ \text { ECL } & \text { Enhanced chemiluminescence } \\ \text { EGFP } & \text { Enhanced Green fluorescence protein } \\ \text { EMA } & \text { European Medicines Agency } \\ \text { ER } & \text { Endoplasmic reticulum } \\ \text { ESI } & \text { Electrospray ionization } \\ \text { FA } & \text { Formic acid } \\ \text { FAD } & \text { Familial Alzheimer's disease } \\ \text { FBS } & \text { Fetal Bovine Serum } \\ \text { FC } & \text { Ferrochelatase } \\ \text { FCS } & \text { Fetal calf serum } \\ \text { FDA } & \text { Food and Drug Administration } \\ \text { FDG } & \text { Fluorodeoxyglucose } \\ \text { FDR } & \text { False discovery rate } \\ \text { FPR2 } & \text { Formyl peptide receptor 2 } \\ \text { FPRL1 } & \text { Formyl Peptide Receptor-Like 1 } \\ \text { FUDR } & \text { 5-Fluoro-2'-deoxyuridine } \\ \text { GAPDHS } & \text { Glyceraldehyde-3-Phosphate Dehydrogenase } \\ \text { GFAP } & \text { Glial fibrillary acidic protein } \\ \text { GFP } & \text { Green fluorescent protein } \\ \text { Glo1 } & \text { Glyoxalase 1 } \\ \text { Gmpr } & \text { Guanosine Monophosphate Reductase } \\ \text { Gng7 } & \text { G Protein Subunit Gamma 7 } \\ \text { GO } & \text { Gene ontology } \\ \text { Gpd1 } & \text { Glycerol-3-Phosphate Dehydrogenase 1 } \\ \text { GSH } & \text { Glutathion } \\ \text { GSK } & \text { Glycogen synthase kinase } \\ \text { GSSG } & \text { Glutathion disulfide } \\ \text { G } \alpha \text { S } & \text { G-protein subunit } \alpha \\ \text { Hapn1/2 } & \text { Hyaluronan and proteoglycan link protein 1/2 } \\ \text { Hb } & \text { Hemoglobin } \\ \text { HBSS } & \text { Hank's Balanced Salt Solution } \\ \text { HBTRC } & \text { Harvard Brain Tissue Resource Center } \\ \text { Hebp1 } & \text { Heme-binding protein 1 } \\ \text { HEPES } & \text { 2-[4-(2-hydroxyethyl)piperazin-1-yl]ethanesulfonic acid } \\ \text { HMOX1 } & \text { Heme oxygenase-1 } \\ \text { Hnrnpl } & \text { Heterogeneous nuclear ribonucleoprotein L } \\ \text { Hnrnpm } & \text { Heterogeneous nuclear ribonucleoprotein M } \\ \text { HPLC } & \text { High-performance liquid chromatography } \\ \text { IB } & \text { Immunoblotting } \\ \text { IBA1 } & \text { Ionized calcium-binding adapter molecule 1 } \\ \text { ICC } & \text { Immunocytochemistry } \\ \text { IgG } & \text { Immunoglobulin G } \\ & \end{array}$




$\begin{array}{ll}\text { Igh-3 } & \text { Ig gamma-2B chain C region } \\ \text { IHC } & \text { Immunohistochemistry } \\ \text { IL-6 } & \text { Interleukin 6 } \\ \text { Ilf2 } & \text { Interleukin enhancer-binding factor 2 } \\ \text { IMAC } & \text { Immobilized metal affinity chromatography } \\ \text { IP } & \text { Immunoprecipitation } \\ \text { IPA } & \text { Ingenuity Pathway Analysis } \\ \text { IR } & \text { Infrared radiation } \\ \text { KO } & \text { Knockout } \\ \text { LC-MS/MS } & \text { Liquid chromatography-tandem mass spectrometry } \\ \text { LDS } & \text { Lithium dodecyl sulfate } \\ \text { LFQ } & \text { Label-free quantification } \\ \text { lincRNA } & \text { Long non-coding ribonucleic acid } \\ \text { Lrba } & \text { LPS Responsive Beige-Like Anchor Protein } \\ \text { LTP } & \text { Long-term potentiation } \\ \text { m/z } & \text { mass to charge ratio } \\ \text { Mag } & \text { Myelin-associated glycoprotein } \\ \text { MAM } & \text { mitochondria-associated ER membranes } \\ \text { MAP } & \text { Microtubule-associated protein } \\ \text { MAPT } & \text { Microtubule-associated protein tau } \\ \text { MARCS } & \text { Myristoylated alanine-rich C-kinase substrate } \\ \text { Mboat7 } & \text { Membrane Bound O-Acyltransferase Domain Containing 7 } \\ \text { MCI } & \text { Mild cognitive impairment } \\ \text { MEM } & \text { Minimal essential medium } \\ \text { MIB } & \text { Mitochondrial intermembrane space bridging supercomplex } \\ \text { MICOS } & \text { Mitochondrial contact site and cristae organizing system complex } \\ \text { miRNA } & \text { Micro ribonucleic acid } \\ \text { MMP } & \text { Mitochondrial membrane potential } \\ \text { MOAC } & \text { Metal oxide affinity chromatography } \\ \text { Mog } & \text { Myelin-oligodendrocyte glycoprotein } \\ \text { MRI } & \text { Magnetic resonance imaging } \\ \text { MRM } & \text { Multiple reaction monitoring } \\ \text { mRNA } & \text { Messanger ribonucleic acid } \\ \text { MS } & \text { Mass spectrometry } \\ \text { Mtx2 } & \text { Metaxin 2 } \\ \text { Mug1 } & \text { Murinoglobulin-1 } \\ \text { NEB } & \text { New England Biolabs } \\ \text { NFT } & \text { Neurofibrillary tangle } \\ \text { NMDA } & \text { N-Methyl-D-aspartic acid } \\ \text { NO } & \text { Nitric oxide } \\ \text { NOS } & \text { Nitric oxide synthase } \\ \text { NP-40 } & \text { Nonidet P40 } \\ \text { NSE } & \text { Neuron specific enolase } \\ \text { PAGE } & \text { Polyacrylamide gel electrophoresis } \\ \text { PBS } & \text { Phosphate-buffered saline } \\ & \end{array}$




$\begin{array}{ll}\text { PCA } & \text { Principal component analysis } \\ \text { PCR } & \text { Polymerase chain reaction } \\ \text { PDGF- } \beta & \text { Platelet-derived growth factor } \beta \text {-chain } \\ \text { PDL } & \text { Poly-D-lysine } \\ \text { PET } & \text { Positron emission tomography } \\ \text { PFA } & \text { Paraformaldehyde } \\ \text { PINK1 } & \text { PTEN-induced putative kinase } \\ \text { PKA } & \text { Protein kinase A } \\ \text { PKC } & \text { Protein kinase C } \\ \text { PMSF } & \text { Phenylmethylsulfonylfluorid } \\ \text { PPIX } & \text { Protoporphyrin IX } \\ \text { PPO } & \text { Protoporphyrinogen oxidase } \\ \text { PrP } & \text { Prion protein } \\ \text { PSD } & \text { Postsynaptic density } \\ \text { PSEN1 } & \text { Presenilin-1 } \\ \text { PSEN2 } & \text { Presenilin-2 } \\ \text { PTM } & \text { Posttranslational modification } \\ \text { RAGE } & \text { Receptor for advanced glycation products } \\ \text { RanBP9 } & \text { RAN Binding Protein } 9 \\ \text { RBFOX1 } & \text { RNA binding protein, fox-1 homolog } \\ \text { RNA } & \text { Ribonucleic acid } \\ \text { RNA-seq } & \text { Ribonucleic acid sequencing } \\ \text { ROS } & \text { Reactive oxidative species } \\ \text { rpAD } & \text { Rappidly-progressing Alzheimer's disease } \\ \text { S100b } & \text { Protein S100-B } \\ \text { SAD } & \text { Sporadic Alzheimer's disease } \\ \text { SAM } & \text { Sorting and assembly machinery complex } \\ \text { SD } & \text { Standard deviation } \\ \text { SDS } & \text { Sodium dodecyl sulfate } \\ \text { SEM } & \text { Stndard error of the mean } \\ \text { Ser } & \text { Serine } \\ \text { sgRNA } & \text { Single guide ribonucleic acid } \\ \text { SNP } & \text { Single nucleotide polymorphism } \\ \text { SnRNP } & \text { Small nuclear ribonucleo proteins } \\ \text { Snrpe } & \text { Small nuclear ribonucleoprotein E } \\ \text { spAD } & \text { Slowly-progressing Alzheimer's disease } \\ \text { Spag9 } & \text { Sperm Associated Antigen } 9 \\ \text { SR } & \text { serine/arginine-rich proteins } \\ \text { SRRM1-2 } & \text { Serine/arginine repetitive matrix protein 1-2 } \\ \text { Stx1 } & \text { Syntaxin 1 } \\ \text { TfR } & \text { Transferrin receptor } \\ \text { TGF- } \beta & \text { Transforming growth factor beta } \\ \text { Thr } & \text { Threonine } \\ \text { Thy1 } & \text { Thymocyte differentiation antigen 1 } \\ \text { TLR } & \\ & \end{array}$




$\begin{array}{ll}\text { Tnc } & \text { Tenascin } \\ \text { TNF- } \alpha & \text { Tumor necrosis factor alpha } \\ \text { Tnik } & \text { TRAF2 and NCK Interacting Kinase } \\ \text { TREM2 } & \text { Triggering receptor expressed on myeloid cells } 2 \\ \text { Tsnax } & \text { Translin Associated Factor X } \\ \text { Vcan } & \text { Versican core protein } \\ \text { Vim } & \text { Vimentin } \\ \text { WB } & \text { Western blotting } \\ \text { WT } & \text { Wild type }\end{array}$




\subsection{Supplementary material}

Supplementary material are available on the $\mathrm{CD}$ attached to this thesis

\section{List of supplementary material:}

\section{Supplementary table 1}

Full list of quantified proteins in the soluble brain fraction of $3 \times \mathrm{Tg}-\mathrm{AD}$ and control mice at each analyzed time point.

\section{Supplementary table 2}

Full list of quantified phosphosites in the soluble brain fraction of $3 \times \mathrm{Tg}-\mathrm{AD}$ and control mice at time points 2 and 18 months.

\section{Supplementary table 3}

List of proteins identified in Hebp1-EGFP Co-IP experiment (Figure 3-17).

\section{Supplementary table 4}

Proteins identified exclusively in one condition (disease or control) at each analyzed time point. 


\section{Acknowledgements}

I would like to thank my $\mathrm{PhD}$ thesis advisor Dr. John Jia En Chua for giving me a chance to pursue my scientific interests. Thank you John for your full trust, responsiveness and kindness!

I would like to express my sincere gratitude to Prof. Dr. Reinhard Jahn for providing me with an opportunity to work at his department and supporting my stay in Göttingen. Thank you Reinhard for your advice and guidance that helped me to become a better scientist!

Many thanks go to my PhD thesis advisory committee members Dr. Dieter Klopfenstein and Prof. Dr. Dirk Görlich for their valuable input during the committee meetings. I am also grateful to Prof. Dr. Ahmed Mansouri, Dr. Halyna Shcherbata and Prof. Dr. Tiago Outeiro for accepting the invitation to join my extended $\mathrm{PhD}$ thesis committee.

My very special thanks go to Dr. Mahdokht Kohansal Nodehi who has not only contributed immensely to the proteomics part of this project but also supported me throughout the entire process. Mahi, thank you for your encouragement and not letting me give up in the darkest moments. Getting to know you was by far the best result of my thesis.

I would like to thank my collaborators without whom this project would not be possible. First and foremost, I thank Dr. Tamara Rabe for her hard work on the immunohistological preparations and responding immediately whenever I needed her help. Many thanks to Prof. Dr. Wolfgang Härtig for providing transgenic mice and always being ready to answer my questions. I thank Dr. Saima Zafar and Prof. Dr. Inga Zerr for providing human brain samples for this study. I would like to acknowledge the lab of Prof. Dr. Henning Urlaub for providing facility for proteomics measurements and particularly Annika Kühn and Monika Raabe for their technical assistance in preparation and measurement of the proteomics samples. I also would like to thank Prof. Dr. Wilfried Kues for generation of induced pluripotent stem cells from AD mice.

I am grateful to amazing Brigitte Barg-Kues for her support and helping me to adapt in the lab at the very beginning of my journey. Many thanks go to Sigrid Schmidt and Ina Ott for their great technical assistance. I also would like to express my gratitude to Dr. Hans-Dieter 
Schmidt for his assistance with administrative procedures but more importantly for the tons of kindness which made the lab a better place.

I am very thankful to Dr. Ulrike Teichmann for the perfect organization of animal facility as well as Thomas Gundlach, Sascha Krause and each and every animal caretaker who worked hard to keep the mice and rats used in this study happy.

I would like to thank everybody in the Department of Neurobiology for providing a great atmosphere over the last four years and for some of the most memorable moments we had together outside the lab. I am also grateful to Ridhima Gomkale, Lorenz Chua and Bishoy Hanna, the very talented rotation students I had a great pleasure to work with.

My special thanks go to Shrutee, Rashi, Claudia, Vedran and Manuel for their help with proofreading this thesis.

I am extremely grateful to Dr. Steffen Burkhardt and Kerstin Grüniger for the excellent organization of the MolBio program. To you and the entire MolBio family, thank you for making me feel at home from the day I arrived in Göttingen!

Finally, I would like to thank my family for their love, understanding and constant support of my endeavors. Your curiosity for science and sincere interest in what I am doing professionally strongly motivated me to carry on with research. I would never be able to achieve so much without you. Thank you! 


\section{Curriculum Vitae}

\section{Personal Information}

Date of birth

Citizenship

Address

Phone

Email
02.10 .1991

Ukraine

Bergenstraße 7

37075 Göttingen

Niedersachsen

+49 157-39172909

oleksandr.yagensky@mpipbc.mpg.de

yagensky@gmail.com

\section{Education}

Since 2014

$2012-2014$

PhD in Molecular Biology, Max Planck Institute for Biophysical Chemistry and Georg-August University of Göttingen, Germany

Department of Neurobiology/Research Group Protein Trafficking in Synaptic Development and Function (Supervisor: Dr. John Chua)

Master of Science (MSc) in Molecular Biology (Final grade A), International Max Planck Research School for Molecular Biology, GeorgAugust University of Göttingen, Germany.

Thesis completed at the Max Planck Institute for Experimental Medicine, Department of Neurogenetics (Supervisors: Prof. Klaus-Armin Nave, Dr. Sandra Goebbels)

2009-2012 Bachelor of Science (BSc) in Biotechnology (Honors), Jagiellonian University in Krakow, Poland

1998-2008 Honors Diploma of Secondary Education at Mykhaylo Kravchuk Gymnasium, Lutsk, Ukraine

\section{Publications}

Increased expression of Hebp1 early in Alzheimer's disease is linked to cytotoxicity. Yagensky 0, Kohansal-Nodehi M, Rabe T, Zafar S, Zerr I, Urlaub H, Härtig W, Chua JJ. Manuscript submitted.

A neuronal PI(3,4,5)P3-dependent program of oligodendrocyte precursor recruitment and myelination. Goebbels S, Wieser GL, Pieper A, Spitzer S, Weege B, Yan K, Edgar JM, Yagensky 0, Wichert SP, Agarwal A, Karram K, Renier N, Tessier-Lavigne M, Rossner MJ, Káradóttir RT, Nave KA. (2017) Nature Neuroscience. 20(1):10-15. 
The Roles of Microtubule-Based Transport at Presynaptic Nerve Terminals. Yagensky 0, Kalantary Dehaghi T, Chua JJ. (2016) Frontiers in Synaptic Neuroscience. 10;8:3. doi: 10.3389.

Heme oxygenase-1 controls an HDAC4-miR-206 pathway of oxidative stress in rhabdomyosarcoma. Ciesla M, Marona P, Kozakowska M, Jez M, Seczynska M, Loboda A, Bukowska-Strakova K, Szade A, Walawender M, Kusior M, Stepniewski J, Szade K, Krist B, Yagensky O, Urbanik A, Kazanowska B, Dulak J, Jozkowicz A. (2016) Cancer Research. 76(19):5707-5718.

Heme Oxygenase-1 Inhibits Myoblast Differentiation by Targeting Myomirs. Kozakowska M, Ciesla M, Stefanska A, Skrzypek K, Was H, Jazwa A, Grochot-Przeczek A, Kotlinowski J, Szymula A, Bartelik A, Mazan M, Yagensky 0, Florczyk U, Lemke K, Zebzda A, Dyduch G, Nowak W, Szade K, Stepniewski J, Majka M, Derlacz R, Loboda A, Dulak J, Jozkowicz A. (2012) Antioxidants and Redox Signaling 16(2):113-27.

\section{Selected Conference Presentations}

2017

2016

2012

2011

2009

2009

Age Dependent Analysis of Brain Proteome in Alzheimer's Disease. Yagensky 0, Kohansal Nodehi M, Rabe T, Zafar S, Zerr I, Mansouri A, Urlaub H, Chua JJE. Poster presentation at Gordon Research Conference on Biology of Aging, Les Diableretes, Switzerland.

A Role of FEZ1 in Phosphorylation-Dependent Protein Transport during Neuronal Polarity Establishment. Yagensky 0, Chua RL, Hanna B, Kohansal Nodehi M, Kalantary Dehaghi T, Chua JJ.

Heme Oxygenase- 1 in Myogenesis: Focus on miRNA. Yagensky 0, Kozakowska M, Ciesla M, Stefanska M, Loboda A, Dulak J, Jozkowicz A. Oral presentation at III International Conference of Biotechnology Students, Krakow, Poland. Won the prize for the best oral presentation in Molecular Biology, Biochemistry and Genetics Session.

Physical and Genetic Interaction between the Essential Kinase Jil-1 and a Novel Protein PW53. Yagensky 0, Regnard C, Becker PB. Poster presentation at Amgen Scholars Symposium at University of Cambridge, United Kingdom.

Age-related heart rate variability changes in adolescents. Yagensky A, Yagensky 0, Sitovskyy A, Yagenska H, Savchuk I. Poster presentation at the $13^{\text {th }}$ Congress of International Society for Holter and Noninvasive Electrocardiology, Yokohama, Japan.

Effect of emotional stress on heart rate variability in healthy adolescents. Yagensky 0, Sitovskyy A, Savchuk I. Oral presentation at $5^{\text {th }}$ International Conference of Students Scientific Society Collegium Medicum UMK in Bydgoszcz, Poland. Awarded with distinction for the second place in the Clinical Session. 


\section{Awards and Honors}

2017

$2012-2013$

$2009-2010$

$2008-2009$

$2008-2009$

2008 $1^{\text {st }}$ prize at the annual PhD student contest of German-Ukrainian Academic Society

Stipend of the Excellence Foundation for the Promotion of the Max Planck Society

Scholar of the Rector of Jagiellonian University

Scholar of the Leonid Kuchma President Foundation "Ukraine"

Scholarship of the President of Ukraine

Bronze Medal at International Biology Olympiad held in Mumbai, India 